\title{
High Resolution Photoelectron Spectroscopy and Femtosecond Intramolecular Dynamics Using Supersonic Molecular Beams
}

\author{
Baohua Niu \\ (Ph.D. Thesis) \\ Department of Chemistry \\ University of California \\ and \\ Chemical Sciences Division \\ Lawrence Berkeley Laboratory \\ University of California \\ Berkeley, California 94720
}

September 1992 
High Resolution Photoelectron Spectroscopy and Femtosecond Intramolecular Dynamics Using Supersonic Molecular Beams

Copyright ㄷ 1992

by

Baohua Niu

The U.S. Department of Energy has the right to use this thesis for any purpose whatsoever including the right to reproduce all or any part thereof 


\section{Table of Contents}

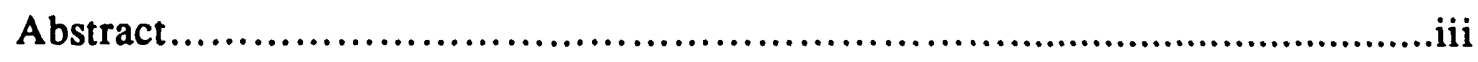

Dedications.......................................................................

Acknowledgments................................................................

Chapter One: Introduction...................................................

A. Photoelectron Spectroscopy and Molecular Electronic Structures........1

B. Born-Oppenheimer Approximation and Vibronic Coupling...............8

C. Intramolecular Dynamics and Autocorrelation Function..................14

References.....................................................23

Figure Caption.......................................................26

Figures.........................................................27

Chapter Two: Experiment......................................................28

A. Description of the MBPES Machine..................................28

B. Maintaining of the MBPES Machine.................................32

C. Tuning \& Calibrating of the MBPES Machine...........................33

1. Tuning the MBPES Machine..........................................33

2. Calibrating the MBPES Machine....................................37

D. Experimental Setups...............................................39

References....................................................44

Tables..........................................................45

Figure Caption......................................................46

Figures........................................................48 
Chapter Three: High Resolution Photielectron Spectroscopy and Femtosecond

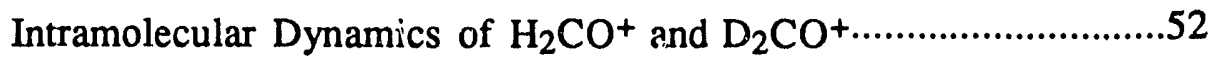

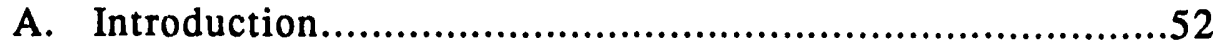

B. Experimental.................................................54

C. Vibronic Coupling and Isotope Effects on Vibronic Coupling...........56

D. The Autocorrelation Function.........................................59

E. Results \& Discussions...............................................62

1. The Ground, $\tilde{\mathrm{X}} 1{ }^{2} \mathrm{~B}_{2}$ state of $\mathrm{H}_{2} \mathrm{CO}^{+}$and $\mathrm{D}_{2} \mathrm{CO}^{+} \ldots \ldots \ldots \ldots \ldots . . . . .63$

2. The Firste Excited, $\widetilde{\mathrm{A}}^{2} \mathrm{~B}_{1}$ state of $\mathrm{H}_{2} \mathrm{CO}^{+}$and $\mathrm{D}_{2} \mathrm{CO}^{+} \ldots \ldots \ldots \ldots \ldots . . .65$

3. The Second Excited, $\widetilde{\mathrm{B}}{ }^{2} \mathrm{~A}_{1}$, state of $\mathrm{H}_{2} \mathrm{CO}^{+}$and $\mathrm{D}_{2} \mathrm{CO}+\ldots \ldots \ldots \ldots . .68$

4. The $\widetilde{\mathrm{C}} 2{ }^{2} \mathrm{~B}_{2}$, state of $\mathrm{H}_{2} \mathrm{CO}^{+}$and $\mathrm{D}_{2} \mathrm{CO}^{+} \ldots \ldots \ldots \ldots \ldots \ldots \ldots . . . \ldots . . . . . . . .70$

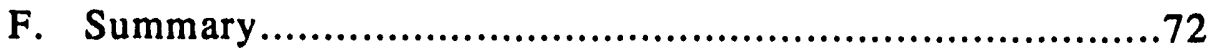

References....................................................74

Tables..........................................................78

Figure Caption.......................................................8

Figures........................................................93

Chapter Four: High Resolution Photoelectron Spectroscopy and Femtosecond Intramolecular Dynamics of $\mathrm{H}_{2} \mathrm{CCO}^{+}$and $\mathrm{D}_{2} \mathrm{CCO}^{+}$.

A. Introduction....................................................111

B. Experimental.................................................113

C. Vibronic Coupling and Isotope Effects on Vibronic Coupling.........115

D. The Autocorrelation Function.......................................118

E. Results \& Discussions...........................................122

1. The Ground $\widetilde{\mathrm{X}}$ state of $\mathrm{H}_{2} \mathrm{CCO}^{+}$and $\mathrm{D}_{2} \mathrm{CCO}+\ldots \ldots \ldots \ldots \ldots . . . . . .122$

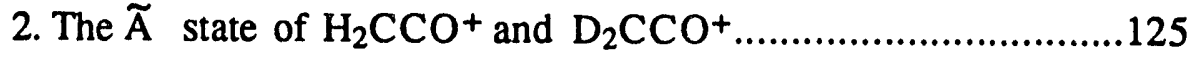




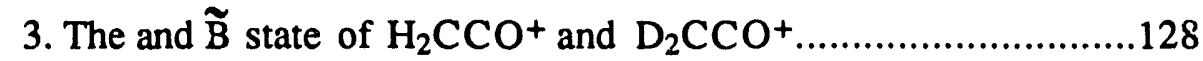

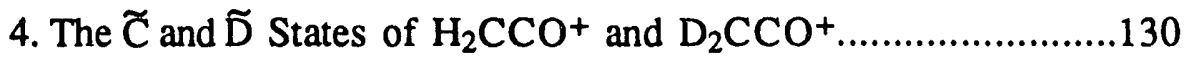

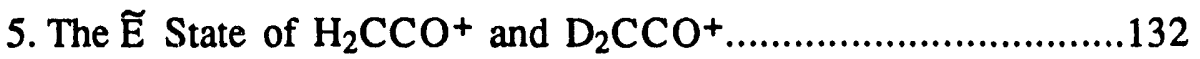

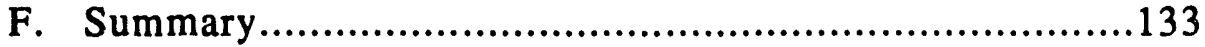

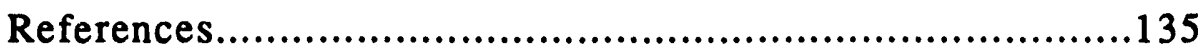

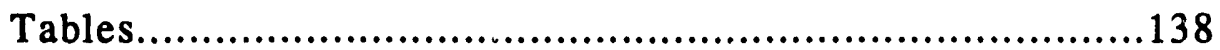

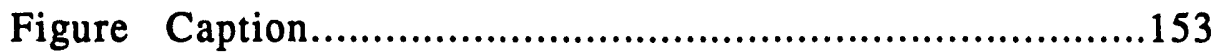

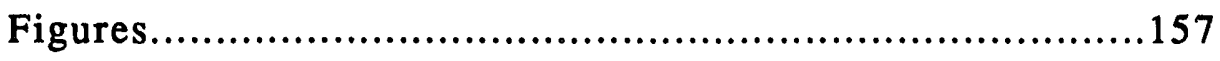

Chapter five: Conclusions, and the Future of High Resolution Photoelectron

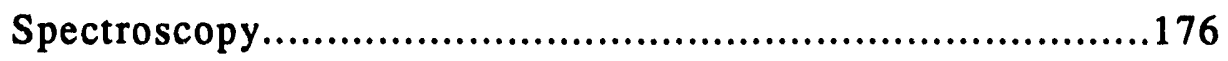




\title{
High Resolution Photoelectron Spectroscopy and Femtosecond Intramolecular Dynamics Using Supersonic Molecular Beams
}

\section{by}

\section{Baohua Niu}

\begin{abstract}
High resolution helium I $\alpha$ photoelectron spectroscopy of formaldehyde and ketene, and their deuterated compounds, are reported. The combination of a unique double-pass high resolution electron-energy analyzer and effective rotational cooling of the sample by supersonic expansion enable the spectroscopic characteristics of these molecular cations to be determined to a much higher accuracy than previously available. The vibrational autocorrelation functions are calculated from the high-resolution photoelectron spectra. They have shed considerable new light into the mechanism of the ultrafast intramolecular dynamics of the molecular cations studied.

The present study reveals much more vibrational structural detail in the first electronic excited state of formaldehyde cations. The first electronic excited state of formaldehyde cations may have non-planar equilibrium geometry. Strong isotope effects on vibronic (vibrational) coupling are observed in the second electronic excited state of formaldehyde. Vibrational autocorrelation functions are calculated for all four observed electronic states of formaldehyde. The correlation function of the first electronic excited
\end{abstract}


state of formaldehyde shows a rather slow decay rate on the femtosecond time scale. The ultrafast decay of the formaldehyde cations in the third electronic excited state implies that dissociation and intramolecular processes are the main decay path ways.

The present spectra of the ground states of ketene cations have more fine structure than previously available. The AIEs of the first and the fifth excited states are determined unambiguously to a much higher accuracy. The doublet-like fine structures present in the first excited state of ketene implies the excitation of a 'soft' mode that was not observed before. The vibrational autocorrelation functions are calculated for four of the six observed electronic states. The dynamics of the ground states of the cations are characterized by a wave packet oscillating with small amplitude around the minimum on the upper PES. The decay dynamics of the first and the fifth excited states of ketene are characterized by ultrafast intramolecular processes like predissociation. 
This Dissertation is Dedicated to the Memories of My Mother: XiouLang Zhang 


\section{Acknowledgment}

Although this dissertation bears my name, there are many people who have made substantial contributions to the research reported. There are people who have gone out of way to extend their warm helping hand to me when I need them most.

First and foremost, I would like to thank my adviser Professor David A. Shirley for his constant support and encouragement during my Berkeley years of study. Dave, you have taught me much more about science, and how to do good science than you realize. I leamed a great deal from his profound knowledge and unparalleled scientific insights. He also improved the scientific soundness of this dissertation considerably. Prof. Yuan T. Lee also deserves special thanks. I first got to know him while he was visiting the Institute of Chemistry in Bejing, 1985. I have benefited tremendously from his incredibly broad knowledge about everything ever since. His encouragement and advice during the most difficult times in my research have put me back on track with confidence many times. Professor Howard Shugart of the physics department at the University of California at Berkeley is thanked, he has carefully read the whole thesis and made many valuable suggestions which improved the integrity of this dissertation considerably.

I spent a very enjoyable year with Professor Mark Johnson's group at Yale University. I have learned a great deal about spectroscopy from Mark. Mark, thank you very much for all the troubles you have taken to help me finding a postdoctoral position and everything else you have done for me. Professor Zheng Gu at Sichuan University, Chengdu, China is my scientific mentor. His encouragement and confidence in me have helped me to be brave enough to come to Berkeley for my graduate studies. My high school teacher Minxi Tan is a true mentor for me. His stimulating teaching style and his devotion to science teaching have aroused my interests in science in general and chemistry 
in particular. Without his early encouragement and guidance, I would have gone nowhere near where I am now.

Dr. Laisheng Wang got me started in the MBPES machine. We have enjoyed many late nights, very long hours experiments together. It has been a great leaming experience for me from the fruitful cooperation. Dr. James E. Pollard, the original designer and builder of the MBPES machine, has helped me enormously in getting the machine back to its best performance. His insight in electron optics and scientific knowledge have helped me overcome many of the difficulties faced me. Dr. Phil Heimann deserves my deep gratitude in many regards, ranging from discussions about science, writing recommendation letters and playing tennis together. I have also enjoyed many helpful discussions with Dr. Alex Schach, who has answered my uncountable numbers of computing questions. Thank you Alex. Mr. Eric Daymo, an undergraduate doing Summer research in the Shirley group in 1991 helped me tremendously to get the formaldehyde experiment started, deserve special thanks. I wish him all the best in his pursuing of an advance degree in Chemistry.

The Shirley group members are like family members to me, always friendly and ready to help. Especially I want to thank Dr. Zahid Hussain, Dr. L. Terminello, Dr. J. Medhurst, Dr. L.Q. Wang, Dr. Y. Zhen, Dr. Tobias Reich, Dr. P. Thalappil, Z.Q. Huang, E. Hudson, T. Huff, E. Moler, S. Kellar and B. Petersen. I want to thank Ms. Tina Peoples for her efforts in keeping the Shirley group running smoothly. She has read the first and second chapters and contributed to the readability of this dissertation. Dr. Harry (Floyd) Davis, J.S. Zhang of Professor Yuan Lee's group, Roger and Ramon of Professor N. . re's group are thanked for their unreserved help when I need them. Roger and Ramon have given me many helps in getting the formaldehyde and ketene generation, purification apparatus to work. 
The supporting staffs at Lawrence Berkeley Laboratory and on the campus machine shops are thanked for their superior efforts in helping getting the science done in a timely fashion. Among them Joseph 'Joe' Katz from the electronic engineering department deserves much more credits than himself knowing them. He always comes up with ideas to solve the electronic, computer problems and fixes them very quickly 'to save' money for the group. Ed. Voroni of the machine shop helped me in more ways than possible to litt here. From finding a right nut to designing new parts, he has always been ki.nd, supportive and helpful in getting my 'fancy' ideas to realities.

My friends here at Berkeley helped me in one way or another for all these years. Among them Mr. Hyman Zipkin and his wife Dr. Xi-Jun Zipkin have always taken me under their arms. They have given me a home away from home where whenever I feel 'depressed' I could go home and forget everything else. The warm home feeling is indescribable in words every time I visit them. Thank you Hy, and thank you Jean.

My deepest gratitude goes to my wife and colleague, Xiaoying, for her love, support and cooperation in many of the long nights she has shared with me in the laboratory trying to finish the experiments on time. Without you, Xiaoying, this thesis would have been impossible to finish today. This thesis is as much yours as mine. I thank my family and my parents in-law for their constant love and encouragement throughout my long 'school' years.

This work was supported by the Director, Office of Energy Research, Office of Basic Energy Science, Chemical Science Division of the U.S. Department of Energy under the Contract No. DE-AC03-76SF00098. 


\section{Chapter One: Introduction}

\section{A. Photoelectron Spectroscopy}

The discoveries of electrons (J.J. Thomson, 1897) and of the photoelectric effect (H.R. Hertz, 1887) in the late nineteenth century had profound influence on the development of the quantum theory of matter. It is Einstein, in his celebrated note of $1905,{ }^{1}$ who first postulated that light radiation itself consists of a beam of corpuscles, the photons, of energy $h v$ ( $h$ is the Plank constant, $v$ the frequency of light) and velocity $c$, and who gave a satisfactory explanation for the photoelectric effect phenomenon in particular. The other solid confirmation of the photon theory of light radiation is the Compton effect (A. Compton, 1924), ${ }^{2}$ which showed that the photon has a momentum equal to $\mathrm{hv} / \mathrm{c}$.

Photoelectron Spectroscopy involves the measurement of the kinetic energies $\mathbf{E}_{k}^{v}$ of the photoelectrons ejected by monoenergetic radiation to study the ionization energies $\mathbf{E}_{\mathrm{I}}^{\mathrm{v}}$; the intensities and the angular distributions of these electrons to elucidate information about the electronic structures of molecules and ions; and the ionization process itself. Since its early development in the 1960's, photoelectron spectroscopy has contributed tremendously to our understanding of the electronic structures of matter and the relationship between electronic structure and chemical activities. 3

In the photoelectric process, the combination of the conservation of energy (as formulated by Einstein) and momentum (as formulated by Compton) gives the following relationship:

$$
h v=E_{l}^{v}+E_{k}^{v}+E_{t}
$$

Here $\mathbf{h v}$ is the incident photon energy, $\mathbf{E}_{\mathbf{l}}^{\mathrm{v}}$ is the electron binding energy, $\mathbf{E}_{\mathbf{k}}^{\mathrm{v}}$ is the photoelectron kinetic energy, $\mathbf{E}_{\mathfrak{l}}$ is the kinetic energy imparted to the ionized matter. The 
superscript $v$ denotes that the energies were referred to the vacuum level energy. Rearranging equation (1.1), incorporating the reference vacuum level energy and other non-ideal effects into one term $I_{\text {const }}$, which stands for the Instrumental constant, we get:

$$
\mathbf{E}_{\mathbf{k}}\left(1+\frac{\mathbf{m}_{\mathrm{e}}}{\mathbf{m}_{+}}\right)=\mathbf{h v}-\mathbf{E}_{\mathrm{I}}-\mathbf{I}_{\text {const }}
$$

Where $\mathbf{m}_{\mathbf{e}}$ stands for the rest mass of electron, $\mathbf{m}_{+}$stands for the mass of the ionized matter. For most photoelectron spectroscopy studies, the following results hold true:

$$
\left(\frac{m_{e}}{m_{+}}\right) E_{k} \ll E_{k} \&\left(\frac{m_{e}}{m_{+}}\right) E_{k} \ll R
$$

Here $\mathbf{R}$ stands for the instrumental energy resolution. Thus the kinetic energy and momentum are carried mostly by the photoelectrons. We can thus simplify equation (1.2) into:

$$
\mathbf{E}_{\mathbf{k}}=\mathbf{h v}-\mathbf{E}_{\mathbf{l}}-\mathbf{I}_{\text {const }}
$$

The constant $\mathbf{I}_{\text {const }}$ can be obtained by calibration of the instrument using rare gases, since the ionization energies (IE) of the rare gases are known precisely from optical spectroscopy.

In a one-electron molecular orbital theory, according to an approximation known as Koopmans' theorem, 2a (the applications of Koopmans' theorem have been discussed in great detail by Richards ${ }^{2 b}$ ) each ionization energy $\left(E_{1}\right)_{j}$ is equal nearly in magnitude to a molecular orbital energy, $\varepsilon_{j}$ :

$$
\left(\mathbf{E}_{\mathrm{l}}\right)_{\mathrm{j}} \cong-\varepsilon_{\mathrm{j}}
$$


By measuring the $E_{k}$, we can get a good estimate of $\left(E_{1}\right)_{j}$. That is the energy levels of the occupied molecular orbitals (typically, a one-electron molecular orbital of the Hartree-Fock determinant) of the neutral molecules can be obtained approximately in experiments with photoelectron spectroscopy.

Beyond this one-electron information about neutral molecules, however, lies a wealth of information concerning structures, spectroscopy, dynamics of the photoions, as well as the dynamics of photoionization process itself. Since most ions other than diatomic ions do not fluorescence, photoelectron spectroscopy has been the primary source of information conceming ion structures and spectroscopic constants. 3,27

In molecular photoelectron spectroscopy, four types of information are available: line energy, line intensity, line shape and the clustering of lines into a band shape. The discrete spectroscopic states that are used to assign the photoelectron spectrum are related to the direct photoionization process by:

$$
M\left(X, v^{\prime \prime}, j^{\prime \prime}\right)+h v \cdots M^{+}\left(\tilde{X}, \tilde{A}, \tilde{B}, \tilde{C}, \cdots v^{\prime}, j^{\prime}\right)+e^{-}
$$

All experiments described in this dissertation were done using supersonic molecular beams. In a supersonic molecular beam experiment the neutral molecule can be assumed to be in its ground electronic, vibrational, and rotational state. Thus the ionization energy spectrum depicts the discrete final states of the photoions with different electronic $(\tilde{\mathbf{X}}, \tilde{\mathrm{A}}$, $\widetilde{\mathrm{B}}, \tilde{\mathrm{C}}_{,} \cdots$ ), vibrational, and rotational excitations. The distribution of the ionic final states is determined through the vertical transitions from the ground electronic, vibrational and rotational state of the neutral molecule to the ionic potential surfaces. Due to the limited resolving power of the currently available photoelectron spectrometer, the rotational excitations could only be resolved for the lightest molecule ion $\mathrm{H}_{2}^{+}{ }^{4}$

The photoionization event itself can be regarded as instantaneous, occurring on a 
time scale $\leqq 10-17$ second 5 [this can be estimated by using the following argument: the molecule usually has a dimension of a few $\AA(3 \AA)$, the speed of light is $\sim 3.0 \times 10^{18} \AA / \mathrm{sec}$, so the photoionization event must occur on a time scale that is $\sim 3 /\left(3.0 \times 10^{18}\right)$ second, and the photoelectron leaves the ion on a time scale of $\tau \approx h / \mathbf{E}_{k}$, for typical $\mathbf{E}_{k} \sim a$ few electron volts $(\mathrm{eV}), \tau \approx 10-16$ second]. Molecular vibration and rotation on the other hand occur on a time scale between $10^{-12}$ to $10^{-14}$ second. This large difference in time scale has its origin from the large difference in the mass between an outgoing photoelectron and the left behind photoion.

This leads to the Franck-Condon ${ }^{6}$ Principle: The electronic transition in a molecule takes place so rapidly in comparison to the vibrational and rotational motion that immediately afterwards, the nuclei still have very nearly the same relative position and velocity as before the transition. ${ }^{7}$ In quantum mechanics language, the Franck-Condon Principle states: The intensity of a vibrational band in an electronically allowed transition is proportional to the absolute square of the overlap integral of the vibrational wavefunctions of the initial and final states. 8 The vibrational wavefunction overlap integral is called the Franck-Condon Factor (FCF).

The direct photoionization process thus can only populate the Franck-Condon regions of the ionic potential energy surfaces (PES), but these regions, usually, extend over several $\mathrm{eV}$, and encompass many vibrational levels of the photoion in a particular electronic state. The shapes of the photoelectron spectrum can provide essential information concerning: the type of orbitals from which the photoelectron is being ejected; the nature of the ionic PES populated; and the dynamics of the photoion intramolecular process. This is illustrated in figure 1 for a typical diatomic molecule $\mathbf{A B}$ in its ground electronic and vibrational state, and $\mathbf{A B}^{+}$in its various electronic and vibrational states. The overall band shapes in the corresponding photoelectron spectra are depicted on the left 
side of the figure.

PES $x$ represents the ground state of the ion that has the same nuclear configuration as in the neutral ground state. Such a configuration invariance is produced by ejection of an essentially non-bonding electron. The resulting photoelectron spectrum shows a very intense $v^{\prime}=0 \leftarrow v^{\prime \prime}=0$ band (this is called the adiabatic ionization energy, AIE) followed by a relatively short progression. Not all molecular ions in their ground states are populated by ejection of non-bonding electrons. Nonetheless, it is very common.

PES a represents an ionic PES in which the ion has a different nuclear configuration (in this case the intermuclear distance $r$ is longer in the ionic state than in the neutral ground state, and usually the photoelectron is ejected from a strong bonding molecular orbital). The corresponding photoelectron band shows a long vibrational progression, and the FCF maximum (this is the vertical ionization energy VIE) appears near the middle of the progression.

In PES b, the Franck - Condon region extends both above and below the dissociation energy limit of the ion. The photoelectron band consists of a progression that converges into a continuum. The onset of the continuum corresponds to the mass spectrometric appearance energies (potentials) of the dissociating fragments.

The transition from the neutral ground state to the photoion state represented by PES c produces an ion in its dissociating region of the PES. The photoelectron band observed is a broad featureless one. However the width of the photoelectron band can give information about the slope of the repulsive region of the PES of the ion.

The PES depicted by curve d represents the crossing or close overlapping of a repulsive PES and a bound PES. If the total wavefunctions of these two approaching curves do not have the same symmetry, the wavefunctions that describe these states become mixed, and they cannot be separated in the Franck-Condon region. The photoion is subject to the lifetime limitation of the repulsive state; this causes 
predissociation. The resulting photoelectron band shows discrete vibrational structures at the very beginning: that is the character of the bound ionic-state vibration in the region below the crossing energy level. Lifetime broadened vibrational structure, according to the Heisenberg Uncertainty Principle, ${ }^{9}$ appears in the region of the crossing (this also causes the electronic predissociation). Depending on the nature of the crossing point, there are two possibilities above the crossing energy level. If the "upper" repulsive state is a very steep and close-lying one, a broad featureless continuum appears in the region above the crossing energy level that bears the character of the repulsive ionic state potential energy surface. If, on the other hand, the repulsive state is relatively further away from the Franck-Condon region, we will again see a resolved vibrational progression which is the continuation of the progression below the crossing energy level.

This example shows that from the photoelectron band shape and simple FranckCondon analysis, we can deduce useful information about the photoions' structure, spectroscopy, and dynamics. The FCF calculations, however, can only be carried out to a fairly satisfactory answer for diatomic molecules. For polyatomic molecules, the FranckCondon factor calculations are feasible only when there are no strongly coupled vibrational modes. It's only recently that polyatomic molecule Franck-Condon analysis has been carried out for very simple molecules like $\mathrm{SO}_{2}+, \mathrm{NH}_{3}+.10$ In the case of formaldehyde, even for the ground ionic state, the Franck-Condon analysis was not satisfactory. ${ }^{10}$ The FCF calculations, up to now, have been confined to the harmonic approximation to treat vibrational wavefunctions. ${ }^{11-13}$ We know that even for diatomic molecules the harmonic approximation is good only at very low levels of vibrational excitations. We have to rely heavily on the direct experimental determination of these properties in place of the Franck-Condon analysis results. In case of strong vibronic coupling, the FCF analysis alone can not give the correct assignment for the spectra obtained. The vibronic coupling effects will be covered briefly in section $\mathbf{B}$ of this chapter. 
To interpret the photoelectron spectrum obtained, one can use the selection rules based on the Born-Oppenheimer approximation 17 (which enables us to treat the electronic and nuclei parts of molecular motions separately), the dipole approximation (for electronic transition) and the harmonic (for vibrational transition) approximation to rule out those inactive vibrational modes. For the details of the selection rules involved in photoelectron spectroscopy, Reference 8 is recommended. Here, only the vibrational selection rules, which are based on the harmonic approximation and are the most important ones for this dissertation, are discussed briefly.

Since the neutral molecule in a supersonic molecular beam is in its vibrational ground state, which is totally symmetric in the molecular symmetry group. Under the harmonic approximation, only the totally symmetric vibrational modes can have non-zero Franck-Condon factors and can be excited to any quanta (even or odd) upon photoionization. Those non-totally symmetric vibrational modes could only be excited with an even number of quanta with non-zero FCFs. But in the case of degenerate vibrations, non-totally symmetric modes could also be excited with an odd number of quanta.

Three factors have to be considered in an attempt to assign the vibrational features observed in the photoelectron spectrum. The first is the anharmonicity of the vibration, which can cause strong mixing of different vibrational modes, and for anharmonic vibrations the selection rules based on harmonic approximation do not necessarily hold on all occasions. Anharmonicity also causes vibrational predissociation. The second factor is that upon ionization many molecular ions produced in a particular electronic state may have a different equilibrium geometry than the neutral molecule in its ground state. This causes some non-totally symmetric (in the neutral molecule) vibrational modes to be excited. The modes that can be excited depend strongly on the differences between the molecule and ion equilibrium geometry in the particular electronic states involved. The third is the aforementioned vibronic coupling effects that have been shown to have great influence on 
the vibrational. structures obtained from photoelectron spectroscopy. ${ }^{18-22}$

The correlations between molecular electronic structure and molecular geometry have been the subject of many studies ever since the very beginning of modern quantum chemistry. The most successful one was introduced by R.S. Mulliken ${ }^{14}$ and further developed by A.D. Walsh ${ }^{15}$ (generally called Walsh diagram) that correlates the molecular orbital energy to some geometric changes in the molecule frame. There are modern $a b$ initial SCF(self-consistent-field) calculations that showed the validity of the original Walsh diagram. ${ }^{16}$ For the details of constructing a Walsh diagram and its interpretations, reference 15 and 16 are recommended. This Walsh diagram approach is used in Chapter 3 to explain the possibility of the non-planar equilibrium geometry of $\mathrm{H}_{2} \mathrm{CO}^{+}$and $\mathrm{D}_{2} \mathrm{CO}^{+}$in their ground and first excited electronic states.

\section{B. Born-Oppenheimer (BO) Approximation and Vibronic Coupling}

In the preceding section, we mentioned the Born-Oppenheimer (BO) approximation 17 which forms the base of our Franck-Condon factor analysis and selections rules. The BO approximation is also known as the adiabatic approximation.

The BO approximation and its limitations can be seen by examining the Schrödinger equation of a diatomic molecule:

$$
\left[\frac{1}{m} \sum_{i}\left(\nabla_{q_{i}}^{2}\right)+\sum_{k} \frac{1}{M_{k}}\left(\nabla_{q_{k}}^{2}\right)+\frac{8 \pi^{2}}{h^{2}}(E-V)\right] \Psi=0
$$

where $q_{i}$ are the coordinates of the electrons with mass $m$ and $q_{k}$ are the coordinates of the nuclei with mass $\mathbf{M}_{\mathbf{k}}$. Suppose the total wavefunction can be written as:

$$
\Psi=\Psi_{r}\left(q_{i}\right) \cdot \Psi_{\mathrm{vr}}\left(q_{k}\right)
$$


Equation (1.6) is the BO approximation, where $\Psi_{\mathrm{e}}$ and $\Psi_{\mathrm{Vr}}$ are the solutions for the equations:

$$
\begin{aligned}
& {\left[\sum_{i}\left(\nabla_{\mathrm{q}_{i}}^{2}\right)+\left(\frac{8 \pi^{2} \mathrm{~m}}{\mathrm{~h}^{2}}\right)\left(\mathrm{E}^{\mathrm{el}}-\mathrm{V}_{\mathrm{e}}\right)\right] \Psi_{\mathrm{e}}=0} \\
& {\left[\sum_{\mathrm{k}} \frac{1}{\mathrm{M}_{\mathrm{k}}}\left(\nabla_{\mathrm{q}_{\mathrm{k}}}^{2}\right)+\left(\frac{8 \pi^{2}}{\mathrm{~h}^{2}}\right) \cdot\left(\mathrm{E}-\mathrm{E}^{\mathrm{el}}-\mathrm{V}_{\mathrm{n}}\right)\right] \Psi_{\mathrm{vr}}=0}
\end{aligned}
$$

respectively. Equation (1.7) is the Schrödinger equation of the electrons moving in the field of the fixed nuclei frame and having a potential of $\mathrm{V}_{e}\left(\mathrm{q}_{\mathrm{i}}\right)$. For each different internuclear distance, $\mathrm{V}_{\mathrm{e}}$ is different. Therefore both $\Psi_{\mathrm{e}}$ and $\mathrm{E}^{\mathrm{el}}$ depend on the internuclear distance as a parameter. Equation (1.8) is the Schrödinger equation of the nuclei moving under the action of the potential $E^{e l}+V_{n}$, where $V_{n}$ is the Coulomb potential of the nuclei.

Let us substitute equation (1.6) into equation (1.5) and use the conditions set by equation (1.7) and (1.8). We can see that equation (1.5) is satisfied only when:

$$
\sum_{k}\left[\frac{1}{M_{k}}\left(\frac{\partial \Psi_{e} \partial \Psi_{v r}}{\partial^{2} q_{k}}\right)+\Psi_{v r}\left(\frac{\partial^{2}}{\partial^{2} q_{k}}\right) \Psi_{e}\right]
$$

is small and can be neglected. That is to say that the variation of $\Psi_{\mathrm{e}}$ with internuclear distance is sufficient slow so that its first and second derivatives can be neglected. We can estimate their magnitude in the following way. Notice that $h \nabla q_{k} \Psi_{e}$ is of the same order as $\mathrm{h} \nabla \mathrm{q}_{\mathrm{i}} \Psi_{\mathrm{e}}$ since the derivatives operate over approximately the same molecular dimensions. $\mathrm{h} \nabla \mathrm{q}_{\mathrm{i}} \Psi_{\mathrm{e}}$ is just the momentum of the electrons, therefore we have:

$$
\frac{\hbar^{2}}{2 M_{k}}\left(\frac{\partial^{2}}{\partial^{2} q_{k}}\right) \Psi_{e} \approx \frac{P_{e}^{2}}{2 M_{k}}=\frac{m}{M} \frac{P_{e}^{2}}{2 m}=\frac{m}{M} E_{e}
$$


Thus, the contribution from (1.9) is of the same magnitude of the electron energy itself times the ratio of electron mass to molecular mass. Even for the lightest diatomic molecule $H_{2}$, we have $m / M \approx 10^{-4}$. For most molecules $m / M \leqq 10^{-5}$ and with typical electron energy in the molecules on the order of $\sim 10 \mathrm{eV}$, this contributes to the electronic energies on the order of $\leqq 0.1 \mathrm{meV}$. Another more physical way of looking at this is to recognize that $\partial \Psi_{e} / \partial q_{k}$ just measures the variation of the electronic wave functions with the nuclear displacements. As long as this variation is small compared with the momentum of the electrons, the BO approximation expressed by equation (1.6) is valid.

In equation (1.6) $\Psi_{\mathrm{vr}}$ is the wave function of a vibrating rotator. To a good approximation it can be expressed as:

$$
\Psi_{\mathrm{VI}}=\left(\frac{1}{\mathrm{r}}\right) \Psi_{\mathrm{V}} \cdot \Psi_{\mathrm{r}}
$$

where $\Psi_{v}$ is the pure vibrational wave function that depends only on the changes of internuclear distance $\mathrm{r}, \Psi_{\mathrm{r}}$ is the rotational wave function depends only on the orientation of the molecule in space. Thus to a first order of approximation, the total wave function of the molecule is:

$$
\Psi=\Psi_{\mathrm{e}} \cdot\left(\frac{1}{\mathrm{r}}\right) \cdot \Psi_{\mathrm{v}} \cdot \Psi_{\mathrm{r}}
$$

This has been shown by Kronig ${ }^{21}$ to hold to a good approximation even when the electron spin and magnetic interaction of angular momentum are included.

From the above discussion, we can see that the $\mathbf{B O}$ or adiabatic approximation represents one of the fundamental starting point of molecular physics and chemistry. It allows the calculation of molecular properties to be divided into two separable parts which 
are much easier to deal with than the original Schrödinger equation (1.5).

In the first part, the electronic wavefunctions are solved keeping the atomic nuclei fixed in space using the Schrödinger equation (1.7). The calculations of electronic structures and wavefunctions for fixed nuclei have been developed to a very high degree of sophistication.

In the second part, the nuclear motion, vibration and rotation can be treated on a given electronic PES using Schrödinger equation (1.8). The vibrational and rotational motions could be further separated using approximation (1.11). The calculation of vibrational and rotational wavefunctions have been constantly pursued to perfection for almost all the molecular systems since the very beginning of molecular physics and chemistry.

We can also see from the above discussion of the BO approximation what are the limitations of this approximation. First, if the variation of $\Psi_{e}$ with internuclear distance is fast so its first or second derivatives with respect to the internuclear distance in equation (1.9) is large and can not be neglected, the BO approximation (1.6) is not valid. This often happens for molecules with degenerate or close-lying electronic states, where very little change in the nuclear configuration causes the energy level to be shifted a lot. Second, if the vibrational and electronic motions of the molecules are strongly coupled, that is to say that the Vibronic effects or coupling is strong, we can no longer separate the electronic and nuclear motions, the BO approximation is not valid. This happens more often than not for highly excited states of molecules. One important consequence of vibronic coupling is the non adiabatic behavior of the nuclear motion they induce; that is to say that the nuclear motion ceases to be confined to a single PES. A lot of very important dynamic processes, such as electronic predissociation and radiationless transition, can only be explained by invoking the vibronic coupling effects.

In general, we can treat the vibronic effect as a perturbations to the $\mathbf{B} \mathbf{O}$ 
approximation, the adiabatic Hamiltonian $\mathscr{K}_{0}$. Although additional periurbations such as vibration-rotation coupling, rotational-electronic coupling (also called Coriolis coupling) and Fermi resonances might also occur in the molecules we study, they are usually not strong enough to have significant effects on the vibrational structures of the photoelectron spectra obtained. The most prominent effects of vibronic coupling on photoelectron spectroscopy that we nave observed are the following:

(i) Vibrational progressions in modes that are not allowed by selection rules base on the $\mathbf{B O}$ and harmonic approximations.

(ii) Very low vibrational frequencies

(iii) Different vibrational progressions in the same electronic state for the isotope substituted compound

There are many excellent discussions in the literature about vibronic coupling and its effects on spectroscopy. 18-24 Only a very brief discussion based on perturbation theory is given here. ${ }^{25}$ We assume that the corrections caused by vibronic coup!ing are small in the sense that perturbation theory still can be used. The molecular Hamiltonian $\mathscr{X}$ can be expanded in a Taylor series in the nuclear coordinates $Q$, the normal coordinates of nuclear vibrations, near the equilibrium configuration $Q_{0}$ :

$$
\mathscr{H}=\mathscr{H}(\mathrm{q} ; \mathrm{Q})_{\mathrm{Q}_{0}}+\sum_{\mathrm{r}=1}^{\infty}\left|\frac{1}{\mathrm{r} !} \frac{\partial^{\mathrm{r}} \mathscr{H}(\mathrm{q} ; \mathrm{Q})}{\partial \mathrm{Q}^{\mathrm{r}}}\right|_{\mathrm{Q}_{0}} \mathrm{Q}^{\mathrm{r}}+\cdots
$$

with corresponding corrections to the energy

$$
\mathbf{E}=\mathbf{E}_{0}+\mathbf{E}_{1}+\mathbf{E}_{2}+\ldots
$$


and corrections to the electronic wavefunctions

$$
\Psi_{\mathrm{e}}=\Psi_{\mathrm{e}}^{0}+\Psi_{\mathrm{e}}^{1}+\Psi_{\mathrm{e}}^{2}+\ldots
$$

The first term in equation (1.13) is the BO approximation Hamiltonian $\mathscr{X}_{0}$. Vibronic couplings can be classified as Linear coupling and Quadratic coupling. Linear coupling refers to the term in $\mathscr{X}$ that is proportional to the nuclear vibration normal coorcinates $\mathrm{Q}_{\mathrm{r}} \mathscr{X}_{1}=\frac{\partial \mathscr{X}}{\partial \mathrm{Q}_{\mathrm{r}}}$; similarly for $\mathrm{Quadratic}$ coupling $\mathscr{X}_{2} \propto \mathrm{Q}^{2}$. Linear coupling is also called Jahn-Teller (JT) coupling if the coupled electronic states are degenerate by symmetry, and pseudo-Jahn-Teller (pJT) coupling or Herzberg-Teller intensity borrowing in ail other cases. Quadratic coupling in linear molecules is called RennerTeller coupling. JT coupling is further classified as dynamic, if the degenerate electronic states keeps its symmetry near the perturbed equilibrium genmetry; or static, if the coupling is so strong that the molecule equilibrium geometry is changed to a lower symmetry thus lift the degeneracy of the initial electronic states.

Since both the Linear and Quadratic vibronic coupling depends on the mass weighted nuclear displacements (normal coordinates) as parameters, it is expected that the couplings are different for isotope substitute compounds in the same electronic state. One consequence of this effect is that it is possible that different vibrational modes can be excited for different isotope substituted compounds. Theoretical calculations have shown that this effect does appear in the isotope substitute compound. ${ }^{26}$ We have observed this effect in the $\mathrm{H}_{2} \mathrm{CO}^{+}$and $\mathrm{D}_{2} \mathrm{CO}^{+}$in both the first and second excited states. 


\section{Intramolecular Dynamics and the Autocorrelation Function}

The photoelectric process is a dynamic one. Under the $\mathbf{B O}$ approximation and the Franck-Condon principle, we can populate a wide range of the ionic PES that was not easy to reach by other means of excitation. The molecular ions created in various ionic states, especially those excited states, undergo interesting dynamic processes: electronic predissociation (ion fragmentation); intramolecular energy redistribution (internal conversion and intramolecular vibrational relaxation); and radiation transition (fluorescence). Very few molecular ions larger than diatomic ions fluorescence, 3,27 indicating that radiationless processes must be the dominant ones in their decay mechanism. The rate and the time scale of these radiationless processes (intramolecular processes) are of great importance in our understanding about the nature and the principles of intramolecular dynamics.

Now with femtosecond lasers relatively easily available, one can, in principle, perform real-time measurements on the details of intramolecular dynamic processes. But, the limited photon energies, fluxes and the inherited band widths $\geqq 0.100 \mathrm{eV}$, limited through the Heisenberg uncertainty principle, make real-time measurements of energy transfer between discrete molecular ionic states very limited. However, an altemative way does exist which is much more versatile and powerful. It is to perform high-resolution measurements in the energy domain. Through the Heisenberg uncertainty principle, energy peak shape can give phenomenological lifetimes for molecular ionic states and the rate of decay of intramolecular dynamics.

As discussed in previous sections, photoelectron spectroscopy is capable of resolving vibrational details of the electronic states of molecular ions. The photoelectron leaves the ion on a time scale of $\tau \leqq 10^{-15}$ second. Thus for those stable ionic states (with life time $\tau \geqq 10^{-12}$ second), the photoelectron experiences essentially a uniform (decaying 
with distance) potential as it travels away from the ionization region and gives rise to inhomogeneous line shapes. For those unstable states of the molecular ion (with life time $\tau$ $\leqq 10-13$ second), a non-uniform (fast varying) potential is experienced by the ejected photoelectron, this generates homogeneously (life time) broadened line shapes.

The finite resolution available (after careful deconvolution of the instrument response function) from the photoelectron spectrometer limits the time for decay over which the photoelectron line shapes are sensitive to $\tau \leqq 2 \times 10^{-13}$ secorids (from the Heisenberg uncertainty principle: $\Delta \mathrm{E} \bullet \Delta \mathrm{t} \geqq \frac{\hbar}{2}, \hbar=0.6582122 \mathrm{eV} \cdot \mathrm{fs}$; this translates to an effective resolution $\leqq 3 \mathrm{meV}$ ). Thus, the outgoing photoelectron carries information about the molecular ion intramolecular dynamics on the femtosecond time scale. A detailed line shape analysis can in principle give us information regarding the ultra fast intramolecular dynamic processes of the molecular electronic states populated by the photoionization process.

Dynamic processes, just like kinetic processes, are often studied using rate expressions involving one or several rate constants. But, to measure a rate constant of intramolecular dynamic process on the femtosecond time scale is not the most productive way of getting information about these processes. An alternative formulation, in which they are described in terms of time autocorrelation functions, is also of great interest and provides complementary information to those obtained by rate expressions. The autocorrelation function gives indications on how long some given system properties persists until they are washed out by dynamic averaging processes. They can, in principle, be calculated from a model. But it is also possible and much more convenient to obtain them directly from experiment measurements, as the Fourier transform of the spectral profile of either an absorption or emission band. This method has been widely used in various field of molecular spectroscopy, in particular NMR, IR, Raman, that have 
provided a wealth of information on intermolecular forces. In ultra fast pulse laser techniques, the autocorrelition function is used to measure the quality and stability of the laser system on the femtosecond time scale. This autocorrelation function approach has proved to be very helpful in getting the qualitative pictures of molecular ion intramolecular dynamics on a femtosecond time scale. $28-34$

The autocorrelation function formalism to interpret the electronic spectroscopy and intramolecular dynamics of molecular electronic excited states was first elucidated by Heller. ${ }^{28} \mathrm{He}$ showed that the Fourier transform of an electronic absorption band profile $I(E)$ was equal to an overlap integral between two nuclear wave functions $\phi(0)$ and $\phi(t)$ :

$$
\left.\langle\phi(0) \mid \phi(t)\rangle=\int_{-\infty}^{+\infty} \frac{I(E)}{E} e^{-i E t / \hbar} d E+\int_{-\infty}^{+\infty} \frac{I(E)}{E} d E\right)
$$

where $\phi(0)$ is the nuclear wave function of the ground state prepared on the PES of an upper electronic staie by a vertical Franck-Condon transition, and $\phi(t)$ is the wave function at time $t$ as it propagates from its initial position on the PES of the upper state. The denominator in equation (1.16) ensures that the correlation function is normalized to unity at time $t=0$. We define a vibrational correlation function $C(t)$ which is equal to the modulus of this overlap integral

$$
C(t)=|\langle\phi(0) \mid \phi(t)\rangle|
$$

and we know that the photoionization cross section $\sigma(E) \propto \frac{I(E)}{E}$.

A.L. Lorquet, J.C. Lorquet et al. in a series of papers 29 first demonstrated that the correlation function as shown in equation (1.16) and (1.17) can also be calculated 
from a photoelectron band with a similar Fourier transform procedure used by Heller. A variation on the method was used by Pollard ${ }^{30}$ et al. to evaluate vibrational correlation function for ethylene ion, and by Reutt ${ }^{31-32}$ et al. for several molecular ions in the small polyatomic molecules limit $\left(\mathrm{H}_{2} \mathrm{O}, \mathrm{D}_{2} \mathrm{O}\right.$ and $\left.\mathrm{C}_{2} \mathrm{H}_{2}, \mathrm{C}_{2} \mathrm{D}_{2}\right)$. J.E. Reutt also has given a fairly detailed derivation of the vibrational correlation functions ${ }^{33}$ (which is used extensively in this thesis). Only a very brief derivation is given here.

Make use of the completeness relation to a set of eige ustates $|E\rangle$ of the molecular Hamiltonian $\mathscr{H}$ :

$$
\int_{-\infty}^{+\infty}\left|\mathbf{E}^{\prime}\right\rangle\left\langle\mathbf{E}^{\prime}\right| d E^{\prime}=1,
$$

and

$$
\int_{-\infty}^{+\infty} \delta(\mathbf{E}-\mathscr{X})\left|\mathbf{E}^{\prime}\right\rangle\left\langle\mathbf{E}^{\prime}\right| d \mathbf{E}^{\prime}=\delta(\mathbf{E}-\mathscr{X})
$$

Express the Dirac $\delta$ function into the right side of equation (1.19)

$$
\int_{-\infty}^{+\infty} \delta\left(E-E^{\prime}\right)\left|E^{\prime}\right\rangle\left\langle E^{\prime}\right| d E^{\prime}=\frac{1}{2 \pi} \int_{-\infty}^{+\infty} e^{i(E-\mathscr{x}) t / \hbar} d t
$$

and we have:

$$
|E\rangle\langle E|=\frac{1}{2 \pi} \int_{-\infty}^{+\infty} e^{i E t / \hbar} e^{-i \mathscr{t} t / \hbar} d t
$$


Consider an electronic transition, under the BO approximation, and make use of the Franck-Condon principle. In the dipole approximation, we could determine the transition intensity by its transition moment:

$$
\left.I(E) \propto E I R_{i f}(E)\right|^{2}=E\left(\bar{M}_{\text {if }}^{\text {el }}\right)^{2}|\langle 0 \mid E\rangle|^{2}
$$

where $\left(\overline{\mathbf{M}}_{\text {if }}^{\text {el }}\right)^{2}$ is the electronic part of the transition moment (assumed to be a constant here); $10>$ and $<E \mid$ are now pure vibrational wave functions of the lower and upper electronic states, respectively. Put equation (1.21) into equation (1.22), and we have:

$$
\frac{I(E)}{E} \propto \int_{-\infty}^{+\infty} e^{i E t / \hbar}\left\langle\phi(0)\left|e^{-i \Re t / \hbar}\right| \phi(0)\right\rangle d t
$$

where $\mathscr{X}$ is the nuclear Hamiltonian of the excited state. When transferred on the PES of the upper state, ket $|\phi(0)\rangle$ represents the initial position of the wave packet at time $t=0$, and the $e^{-i \mathscr{t} t / \hbar}|\phi(0)\rangle$ represents the wave packet at time $t$ as it propagates on the upper (ionic) PES. Then:

$$
\frac{\mathrm{I}(\mathrm{E})}{\mathrm{E}} \propto \int_{-\infty}^{+\infty} \mathrm{e}^{\mathrm{iEt} / \mathrm{h}}\langle\phi(0) \mid \phi(t)\rangle d t
$$

An inverse Fourier transformation and normalization gives us equation (1.16) and (1.17).

$$
\begin{aligned}
& \left.\langle\phi(0) \mid \phi(t)\rangle=\int_{-\infty}^{+\infty} \frac{\mathrm{I}(\mathrm{E})}{\mathrm{E}} \mathrm{e}^{-i \mathrm{E} / / \hbar} \mathrm{dE}+\int_{-\infty}^{+\infty} \frac{\mathrm{I}(\mathrm{E})}{\mathrm{E}} \mathrm{dE}\right) \\
& \mathrm{C}(\mathrm{t})=|\langle\phi(0) \mid \phi(t)\rangle| .
\end{aligned}
$$


By measuring the photoionization cross section for the electronic state we are interested at a discrete energy, applying the appropriate Fourier transform method, we can calculate the correlation function for that electronic state. The correlation function so obtained permits us to gain information about intramolecular dynamics of a wave packet composed of discreet adiabatic (in the BO sense) eigenstates as they evolve on the excited state PES on the femtosecond time scale.

The vibrationally resolved photoelectron spectroscopic features can in this formalism be interpreted as having their origin from the ultra fast intramolecular dynamics. By the Heisenberg uncertainty principle, the broadest feature of the spectrum obtained, the photoelectron bandwidth, gives the time resolution in the correlation function. For a typical photoelectron band width of one electron volt or so, the time resolution is about a few tenths of a femtosecond. The narrowest feature, limited by the instrumental energy resolution, defines the time window over which the autocorrelation function is meaningful. A resolution of $10 \mathrm{meV}$ FWHM gives a time window of $0 \sim 70$ femtosecond. But, careful deconvolution of the instrument response function; elimination of rotational and sometimes spin-orbital contributions to the vibrational autocorrelation function; an effective resolution of $<3 \mathrm{meV}$ was achieved. This extends the time window to $\sim 200$ femtoseconds for those stable states.

To evaluate the vibrational correlation function, we have to determine $\sigma(E)$ vs. $E$ from $I(E)$ vs. $E$ which we measure in photoelectron spectroscopy, since $\frac{\sigma(E)}{E} \propto \frac{I(E)}{E}$. This is accomplished by deconvoluting the instrument response function $\mathrm{I}(\mathrm{E})_{\text {ir }}$, which is determined from the photoelectron spectra of a rare gas with ionization potential comparable to the band of interest. The contributions of the finite rotational temperature in a supersonic molecular beam experiment (typically $5 \sim 10$ Kelvin) and the rotational excitations' contribution to the vibrational correlation function were eliminated by convoluting $I(E)_{\text {ir }}$ 
with a gaussian function $\mathrm{I}(\mathrm{E})_{\mathrm{g}}$ to generate a rotationally broadened instrument response function. The width of the gaussian function was chosen from the average rotational constant, $\mathbf{B}_{\mathbf{a v g}}$, of the molecular ion for the particular electronic state (if available), the finite rotational temperature and the rotational selection rules for photoionization.

The vibrational correlation functions were determined by the following procedure. Each photoelectron band was isolated and the empirically determined background plus any constant background was removed. The resulting band was then normalized to give $\mathrm{I}(\mathrm{E})$, and Fourier transformed using a discrete FFT ${ }^{35}$ procedure. The combined effects of the instrument response function and rotational broadening were then removed. This is achieved by dividing the separate FFT of the $I(E)_{\text {ir }}$ (normalized) and $I(E)_{g}$ (normalized) into the FFT of the $I(E)$. The modulus of the quotient is then evaluated, giving the vibrational correlation function. The method is summarized:

$$
\begin{aligned}
& \operatorname{Crib}^{\mathrm{vi}}=|\langle\phi(0) \mid \phi(t)\rangle| \propto \\
& \left|\int_{-\infty}^{+\infty} I(E) e^{-i E t / \hbar} d E\right| \div \\
& \left|\int_{-\infty}^{+\infty} I(E)_{i r} e^{-i E t / \hbar} d E \times \int_{-\infty}^{+\infty} I(E)_{g} e^{-i E t / \hbar} d E\right|
\end{aligned}
$$

For those states that have spin-orbital splittings, which introduces an oscillatory factor in the vibrational correlation function, additional correction must be made to remove this oscillatory contribution. This effect is removed by dividing the correlation function by $|\cos (\Omega t / 2 \hbar)|$, as suggested by Lorquet et al.. ${ }^{29 b}$ The value of $\boldsymbol{\Omega}$ is selected close to the spin-orbital splitting of the element that makes the most contribution to the splitting. 
Very recently, F. Remacle et al., ${ }^{34}$ have suggested that there exists a relationship between the vibrational correlation function $C(t)$ and the population decay function $\mathbf{P}(t)$ for the particular electronic state we are interested. They defined an average population decay curve $\mathbf{P}_{\mathrm{av}}(\mathrm{t})$ valid in a particular time period, and $\mathbf{C}_{\mathrm{av}}(\mathrm{t})$ the average correlation function. It was pointed out by these authors that for a specific excitation, the exact initial rate of decay (valid up to the dephasing time $T_{1}$ ) is equal to the initial slop of $|C(t)|^{2}$, and the subsequent time evolution can be obtained by averaging $|C(t)|^{2}$ over its oscillation. This generates $|C(t)|_{a v}^{2}$ whose area (from $T_{1}$ onwards) is directly related to an average decay lifetime. At time $t>T_{1}$, to a good approximation, the average decay curve $\mathbf{P}_{\mathrm{av}}(\mathrm{t})$ can be derived by multiplying $|C(t)|_{a v}^{2}$ with an appropriate constant. This method is adopted to estimate some of the ionic-states average decay lifetimes.

The vibrational correlation function, described in the preceding paragraphs, is a very sensitive measure of the stability of a vibrational wave packet on a PES; through which we can get both qualitative and quantitative information about the decay and dynamics of the ionic excited states PES. And we can gain valuable insight into the ultra fast decay mechanism of the molecular ions on highly excited electronic states on the femtosecond time scale.

In this dissertation the helium $\mathrm{I} \alpha$ photoelectron studies of the spectroscopy and intramolecular dynamics of a series of molecular ions are presented. The systems studied: $\mathrm{H}_{2} \mathrm{CO}^{+}, \mathrm{D}_{2} \mathrm{CO}^{+} ; \mathrm{H}_{2} \mathrm{CCO}^{+}, \mathrm{D}_{2} \mathrm{CCO}^{+}$, have a long history of both experimental and theoretical interests. The electronic spectroscopy of these ions in all of the electronic states accessible by the Helium I $\alpha$ radiation, however, is very limited. The unimolecular decomposition of these systems has proven to be very rich. Especially, very little is known and much ambiguity remains concerning the intramolecular dynamics of the electronic excited states of these molecular ions. 
One of the major focuses of this dissertation is the effective coupling of supersonic molecular beam sampling and a unique double-pass high resolution electron energy analyzer that enables us to obtain much higher resolution photoelectron spectra than was previously available. Many features of the vibrationally resolved spectra of the ions are elucidated here for the first time, and much refined and previously unknown spectroscopic constants are reported. The observation of strong isotope effects on vibronic coupling further confirmed earlier theoretical calculations that great care must be taken to interpret spectra of isotope substituted molecules. The vibrational correlation formalism, as briefly discussed in this introduction, has been applied to the photoelectron bands. The femtosecond intramolecular dynamics of these molecular ions have been studied experimentally for the first and have provided future theoretical works with much needed experimental data.

The remainder of this dissertation is organized as follows: Chapter Two gives a description of the experimental techniques that are common to all of the work in this dissertation. The Helium I $\alpha$ photoelectron spectroscopy and the intramolecular dynamics of formaldehyde and ketene as well as their deuterium substituted molecules are presented separately in two subsequent chapters. Chapter Three presents the $\mathrm{H}_{2} \mathrm{CO}^{+}$and $\mathrm{D}_{2} \mathrm{CO}^{+}$ system. The $\mathrm{H}_{2} \mathrm{CCO}^{+}$and $\mathrm{D}_{2} \mathrm{CCO}^{+}$systems are presented in Chapter Four. In the final chapter, the conclusion and a personal verisimilitude of the future of high resolution photoelectron spectroscopy are discussed. 
References:

1. A. Einstein, Ann. Phys. 17, 132 (1905).

2a. T. Koopmans, Physica, 1, 132 (1934).

2b. W.R. Richards, Int. J. Mass Spectr. \& Ion. Phen.,2, 419 (1969).

3a. J.P. Maier, ibid, 104, 1-22 (1991).

3b. K.P Hube, G. Herzberg, Molecular Spectra and Molecular Structures IV, Constants of Diatomic molecules, Van Norstrand Reinhold Co., (1979).

4. J.E. Pollard, Ph.D. Thesis, Department of Chemistry, The University of California at Berkeley (1982).

5. H.A. Bethe, R. Jackiew, Intermediate Ouantum Mechanics, Benjamin Cummings Pub. Co., (1986)

6a. J. Franck, Trans. Faraday Soc.,21, 536 (1925).

6b. E.U. Condon, Phys. Rev., 32, 858 (1928).

7. G. Herzberg, Molecular Spectra and Molecular Structures I.Diatomic Molecules, Van Norstrand Reinhold Co., p 194-203 (1950).

8. J.W. Rabalais, Principles of Ultraviolet Photoelectron Spectroscopy, John Wiley \& Sons., NY., p53-54 (1977).

9. A. Messiah, Quantum Mechanics, Vol. I, p320, John Wiley \& Sons., New York. (1976).

10. D.C. Moule, in Vibrational Spectra \& Structures, ed. by J.R. Durig, Elsevier Sci. Pub. Com., p228-271 (1977).

11a. L.S. Cederbaum, W. Domcke, J. Chem. Phys., 64(2), 603-611 (1975).

11b. W. Domcke, L.S. Cederbaum, ibid, p612-625 (1975).

12. K. Takeshita, J. Chem. Phys., 24(11), 7259-7265 (1991).

13. K. Takeshita, ibid, 96(2), 1191-1209 (1992). 
14. R.S. Mulliken, Rev. Mod. Phys., 14, 204 (1942).

15a. A.D. Walsh, J. Chem. Soc., 2260 (1953).

15b. D.C. Moule, A.D. Walsh, Chem. Rev., 75(1), 67-84 (1975).

16. R.J. Buenker, S.D. Peyerimhoff, Chem. Rev., 74(2), 127-188 (1974).

17a. M. Born, J.R. Oppenheimer, Ann. Phys.(Lepzig), 84, 457-484 (1927).

17b. M. Born, Nachr. Akad. Wiss. Gottingen, Math.-Phys. KL, Ila, P1 (1951).

17c. M. Born, K. Huang, Dynamics of Crystal Lattice, Oxford U. Press, NY., (1954).

18. R. Englman, The Jahn-Teller Effect in Molecules and Crystals, John Wiley \& Sons Interscience, New York (1972).

19. G. Fisher, Yibronic Coupling, Academic Press, Orlando (1984).

20. I.B. Bersuker, V.Z. Polinger, Vibronic Interactions in Molecules and Crystals, Springer-Verlag, New York (1989).

21. H. Koppel, W. Domcke, and L.S. Cederbaum, Adv. Chem. Phys., 57, 59 (1984)

22. R.L. Wheatten, G.S. Ezra, and E.R. Grant, Annul. Rev. Phys. Chem., 36, 277 (1985).

23. M. Kasha, Discuss. Faraday Soc., 2, 14 (1950).

24. J.P. Maier, in Kinetics of Ion-Molecule Reactions, ed. by P. Ausluos Plenum, New York, P437 (1979).

25. A. Messiah, Quantum Mechanics, Vol. II, John Wiley \& Sons Interscience, New York (1976).

26a. L.S. Cederbaum, and W. Domcke, J. Chem. Phys., 64(2), 603 (1975).

26b. W. Domcke, and L.S. Cederbaum, ibid, 64(2), 612 (1975).

27. J.P. Maier ed., Ion and Cluster Ion_Spectroscopy and_Structure, Elsevier Scientific Publishers B.V. (1989).

28a. E.J. Heller, J. Chem. Phys., 68, 2066 (1978).

28b. E.J. Heller, E.B. Stechel, M.J. Davis, ibid, 73(10), 4720 (1980). 
28c. E.J. Heller, Acc. Chem. Res., 14, 368 (1981).

28d. E.J. Heller, R. Sundberg, and D. Tanner, J. Chem. Phys., 76, 1822 (1982).

29a. B. Leyh-Nihaut, and J.C. Lorquet, ibid, 88(9), 5606 (1988).

29b. A.J. Lorquet, J.C. Lorquet, J. Delwiche, and M.J. Hubin-Franskin, ibid, 76(10), 4692 (1982).

30. J.E. Pollard, D.J. Trevor, J.E. Reutt, Y.T. Lee, and D.A. Shirley, ibid, 81(12), 5302 (1984).

31. J.E. Reutt, L.S. Wang, J.E. Pollard, D.J. Trevor, Y.T. Lee, and D.A. Shirley, ibid, 84(6), 3022 (1986).

32. J.E. Reutt, L.S. Wang, Y.T. Lee, D.A. Shirley, ibid, 85(12), 6928 (1986).

33. J.E. Reutt, Ph.D. Thesis, Department of Chemistry, The University of California at Berkeley (1986).

34. F. Remacle, M. Desoute-Lecomte, and J.C. Lorquet, J. Chem. Phys., 21(7), 4155 (1989).

35. E. Oran Brighan, The Fast Fourier Transform, Prentice-Hall, Englewood Cliffs, 1974, p164 
Figure Captions:

Figure 1. A typical diatomic molecule $\mathbf{A B}$ in its electronic and vibrational ground state PES, and the molecular ion $\mathbf{A B}^{+}$created by direct photoionization in its various electronic and vibrational states PES. Depicted at the right side of the figure is the photoelectron spectrum of the corresponding PES. For details concerning the shape of the photoelectron band, see main text. 


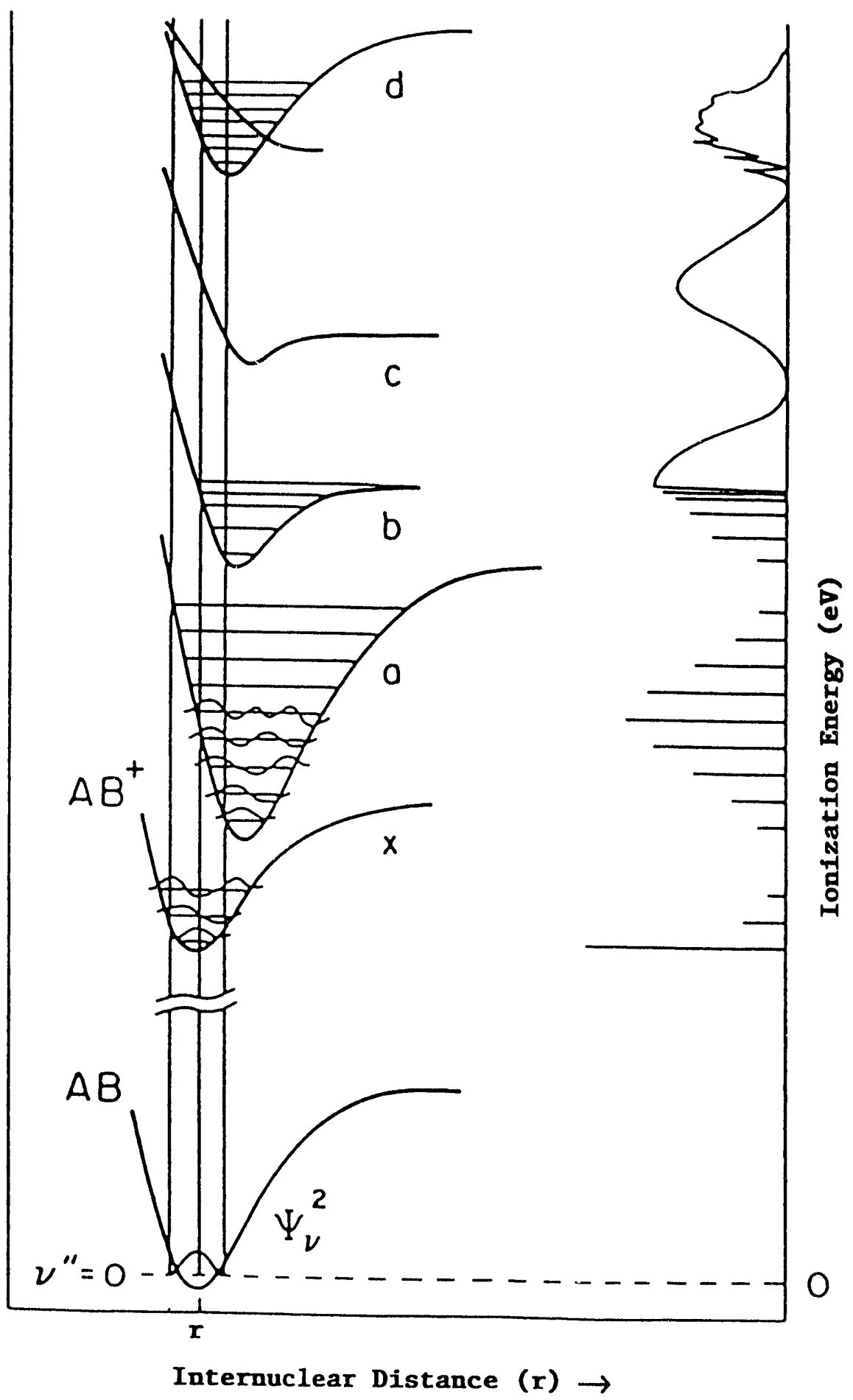

Figure 1 


\section{Chapter Two: Experimental}

The apparatus and experimental method common to all chapters of this dissertation will be described in this chapter. Four sections will each describe the apparatus; maintenance of the apparatus; tuning and calibration of the apparatus; and the experimental conditions used for all the experiments reported in this dissertation.

The apparatus that is essential to this dissertation had been documented in great detail by four Ph.D. dissertations from this group. ${ }^{1-4}$ The first section of this chapter covers the apparatus very briefly. Maintenance, tuning and calibration of the spectrometer are essential to the success of any experiment. These have not been explained at any length previously. They are detailed in the second and the third section, to facilitate future, more productive use of the apparatus. In the last section, experimental conditions used for all the experiments contained in this dissertation are described together, with detailed procedures to prepare and purify the samples.

\section{A. Description Of The MBPES Machine}

The Molecular Beam Photoelectron Spectrometer (MBPES) used for this dissertation had been described in great details in James E. Pollard, ${ }^{1}$ Dennis J. Trevor, ${ }^{2}$ Janice E. Reutt ${ }^{3}$ and Laisheng Wang's Ph.D. ${ }^{4}$ theses. It will only be covered briefly here for completeness.

\section{Vacuum System and Beam Source Chamber}

The MBPES apparatus as shown in Figure 1 is based on a 16-inch diameter stainless steel six-way cross for the main chamber. It is accessible to the supersonic molecular beam source; the high-resolution electrostatic electron-energy analyzer; the quadrupole mass spectrometer; and the helium discharge lamp. These four flange-mounted units are equipped with differential pumping capabilities. The main chamber is pumped by 
two $\mathrm{LN}_{2}$ baffled diffusion pumps (Varian VHS-6) with a combined pumping speed of 1800 liters per second.

The beam source consists of a $70 \mu \mathrm{m}$ diameter nozzle (which can be changed) and a $0.858 \mathrm{~mm}$ diameter, $6.4 \mathrm{~mm}$ tall skimmer (the skimmer can be changed), with a normal nozzle-to-skimmer distance of $D_{n s}=6.4 \mathrm{~mm}$ (adjustable to maximize beam intensity without compromising resolution), pumped by an unbaffled 4000 liters per second diffusion pump (Varian VHS-10) backed by a 1000 liters per second Roots blower (Edwards EH-1200) and an $80 \mathrm{cfm}$ mechanical pump (Edwards E2M-80). The nozzle assembly can be cooled by a $\mathrm{LN}_{2}$ filled jacket and heated tip to $4.00{ }^{\circ} \mathrm{C}$ by a noninductively wound Nichrome heating cable. Under typical runaing conditions (for experiments reported in this dissertation) with a stagnation pressure of 700 torr at $25^{\circ} \mathrm{C}$, the beam s surce chamber pressure is $<5 \times 10^{-5}$ torr. The main chamber pressure is $<2$ x 10-6 torr which is an absolutely necessary condition to eliminate the problem of photoelectrons being scattered by the background molecules.

\section{High Resolution Electrostatic Electron Energy Analyzer}

The electron energy analyzer consists of four major components: the electrostatic lenses; the $90^{\circ}$ sector analyzer; the hemispherical analyzer and the multichannel detector. The entire analyzer is connected to the main chamber via a $1.79-\mathrm{mm}$ diameter aperture through which the photoelectron travels. The photoelectron first encounters two asymmetric three-element cylindrical electrostatic lenses. The first three-element lens sets the fixed pass energy at which the photoelectrons travel through buth the $90^{\circ}$ sector analyzer (8.00 eV at present) and the hemispherical analyzer $(1.000 \mathrm{eV}$ at present). The second three-element lens performs the actual scan of electron kinetic energies and focuses the photoelectrons at the entrance of the $90^{\circ}$ sector analyzer. The $90^{\circ}$ sector analyzer was intended to reduce the background. The photoelectrons that emerge from the exit aperture (1.79 $\mathrm{mm}$ at present) of the $90^{\circ}$ sector analyzer travel through another three-element 
asymrnetric lens that decelerates the electrons to $1.000 \mathrm{eV}$ and focuses the electrons at the entrance to the hemispherical analyzer. The photoelectrons are then dispersed according to their kinetic energies and detected by the detector, which consists of a pair of $40 \mathrm{~mm}$ active diameter MCP in chevron configuration, a resistive anode, and position-encoding electronics.

The analyzer chamber is pumped by two $\mathrm{LN}_{2}$ baffled diffusion pumps (Varian VHS-4) with a combined pumping speed of 920 liters per second. The differential pumping arrangements of the analyzer chamber ensure that the pressure in the chamber is < $2 \times 10^{-8}$ torr with the molecular beam running. This differential pumping is absolutely necessary to maintain the highest resolution obtainable, a constant dispersion function of the analyzer, and the protection of the analyzer surfaces and detector assembling.

The resolution obtainable depends strongly on the pass energies of the $90^{\circ}$ sector, and the hemispherical analyzers. For experiments described in this dissertation, the pass energies were set at $8.00 \mathrm{eV}$ for the $90^{\circ}$ sector, and $1.000 \mathrm{eV}$ for the hemispherical analyzers. Under these conditions, the highest resolution achieved was $9.6 \mathrm{meV}$ (FWHM) as measured by $\mathrm{Ar}^{+}{ }^{2} \mathbf{P}_{1 / 2},{ }^{2} \mathbf{P}_{3 / 2}$ peaks.

\section{The Quadrupole Mass Spectrometer (QMS)}

The quadrupole mass spectrometer (Extranuclear Laboratory) has been used extensively to perform molecular beam diagnosis. The QMS consists of a quadrupole mass filter, ion optics, RF electronics, and a detector consisting of a spiraltron multiplier and a current amplifier.

The QMS is connected to the rnain chamber via a $6.4 \mathrm{~mm}$ aperture through which the ions are transmitted. The QMS housing is differentially pumped with a 500 liters per second turbomolecular pump and typically maintains pressure of $<5 \times 10^{-8}$ torr with the molecular beam running. 
The current setups of the QMS and the electron energy analyzer are not suitable for photoelectron-photoion coincidence experiments. For photoelectron spectra described in this dissertation, the QMS was not operated. But it was used to do molecular beam diagnosis and to optimize the molecular beam conditions used for these experiments.

\section{The Photon Source}

A windowless, differentially pumped cold cathode rare gas DC discharge lamp was used for all the experiments in this dissertation. The lamp operating conditions are optimized to produce the helium I $\alpha(584.3340 \AA, 21.21734 \mathrm{eV})$ resonance line. At typical operating conditions of 1.0 Torr helium and $180 \mathrm{~mA}$ discharge current, the lamp produces a photon line width of $<2 \mathrm{meV}$ (estimated) and a photon fluxes around $10^{13}$ photons per second as measured by a home-built copper photediode. The radiation distribution of the lamp, as measured by the photoelectron spectra of Argon, was found to be $97.2 \%$ helium I $\alpha(584.3340 \AA, 21.21804 \mathrm{eV}), 2.3 \%$ helium $\mathrm{I}_{\beta}(537.02915 \AA, 23.08470 \mathrm{eV}), 0.4 \%$ helium $I \gamma(522.2128 \AA, 23.74209 \mathrm{eV})$, and $<0.1 \%$ of higher resonance lines. Under most circumstances, the contributions from radiation lines other than helium I $\alpha$ could be neglected. But they must be taken into account when in doubt about peak assignments.

In principle, the rare-gas discharge radiations of argon, reon, krypton and xenon can also be used. The natural linewidths of the principal I $\alpha$ lines are narrower, and the discharge can be achieved at much lower lamp pressures than for helium. This reduces the pressure-broadening contributions to the photon linewidth, but the radiation produced from higher resonance lines is much greater. Unless monochromatization without substantial loss of the photon flux can be performed, these rare-gas radiation lines have very limited application. They were not used for any of the experiments in this dissertation. 


\section{B. Maintenance of the MBPES Machine}

In this section, the maintenance of the MBPES machine is discussed. Since this topic has not been covered in any length by previous publications, every detail is given, to help future researchers who will use the MBPES machine.

The MBPES machine, when operated under normal conditions, requires very little maintenance if any at all. The major concern is to keep the machine in its best working condition that depends strongly on the cleanness of the main chamber and the analyzer chamber, on the stability of the electronics and power supplies that power and control the voltages for all the lenses and the analyzers. It is preferable if possible to keep the system under vacuum and keep the analyzer chamber pressure at $<2 \times 10^{-8}$ torr at all times. The power supplies and electronics are left on always to avoid troubles of "warming" up and other interruptions. To minimize power-line-voltage fluctuations during normal operations, the MBPES machine has its own isolation transformer on its main power lines which supplies isolated power to the MBPES electronics system. An isolated 'clean' grounding poin $\hat{\imath}$ is used for all grounding. The mechanical pumps of the MBPES machine are powered from a different power source. The last two conditions are essential in a "noisy" environment like its current location.

Routine maintenance was done by first purging the system vacuum with dry Argon or dry $\mathrm{N}_{2}$ from the analyzer chamber slowly, then opening up the system from the beam source flange; and disengaging the beam source chamber from the main chamber. It is advisable to clean the main chamber (the $\mu$-metal) with "Kimwipe" papers soaked with acetone first; followed by "Kimwipe" papers soaked with alcohol; and finally to wipe the surfaces dry of any solvent traces (do not try to use a heat gun to dry the surfaces). It is usually a good idea to cover up the aperture of the analyzer chamber that extends into the main chamber, to avoid any possible contamination of the analyzer chamber during routine cleaning of the main chamber. 
The beam source chamber is usually contaminated with diffusion pump fluid, since the diffusion pump used to evacuate the beam source does not have any kind of baffle. This is the major contaminating source under most operating conditions. It has to be cleaned after continuing use for about a month or whenever you suspect that the beam source is too dirty.

It is cleaned by first wiping out most of the pump fluid found in the beam source chamber and beam reducer; followed by removing the nozzle skimmer assembly; sonicating the nozzle aperture and the skimmer with acetone first and then alcohol. The nozzle aperture and the skimmer are then placed in an electric oven heated to around $150^{\circ} \mathrm{C}$ for twenty minutes before re-assembling. Before connecting the beam source chamber to the main chamber, check the VHS-10 diffusion pump fluid level, add additional pump fluid if necessary to maintain full pumping speed.

\section{Tuning and Calibration of the MBPES Machine \\ 1. Tuning of the MBPES Machine}

Tuning up the MBPES machine will be necessary whenever the resolution achievable is degraded. At this writing, the causes of this 'degradation' are still not fully understood, but it does seem to correlate with the following conditions: voltages drift of the lens elements and analyzers; the changing of the surface conditions in the main chamber and analyzer chamber usually accompanied by changes in the kinetic energy offset values; changing operating conditions of the lamp; deterioration of the $\mu$-metal magnetic shielding properties. A detailed tuning-up procedure will be described in this section.

\section{a. Overview}

Tuning up the MBPES machine usually involves checking the conditions of the system cleanliness; the functioning of the lamp; the adjustments on the voltages of various lenses and analyzers to achieve the best resolution. This can be accomplished by running 
an about 20 torr Argon beam and changing the $V_{k}$ (kinetic energy of the photoelectrons) settings to place the $A r^{+2} \mathbf{P}_{1 / 2}$ or $2 \mathbf{P}_{3 / 2}$ peak at the center of the left or right half of the detector. Do a normal mode scan, make adjustments and check the changes in resolution and transmission.

It is always good practice to keep the MBPES machine system as clean as possible by maintaining a good vacuum in the main chamber (with no molecular beam running, its base pressure is $<8.0 \times 10.8$ torr) and the analyzer chamber (with no molecular beam running, its base pressure after a good bakeout (at $100^{\circ} \mathrm{C}$ for 48 hours) is $<1.0 \times 10^{-8}$ torr. Since contamination from the beam source is unavoidable under prolonged operation, it's highly recommended that the beam source chamber and main chamber be cleaned periodically. While cleaning the main chamber, check the residual magnetic field inside the chamber with a gaussmeter. It should give the following readings: vertical $\sim 50$ mGauss, north-south $\sim 15$ mGauss, and east-west $\sim 10$ mGauss at the center of the main chamber (near the ionization region).

\section{b. Cleaning and Reconditioning of the Lamp}

With clean beam source and main chambers, the next step is to make sure that the lamp is functioning properly. This can be done by operating the lamp at different conditions and check for changes in resolution. This is very sensitive especially at lower lamp pressures. If the lamp is functioning properly, at 1.5 torr Helium and $200 \mathrm{~mA}$ discharge current, the resolution is $1 \mathrm{meV}$ (as measured with $\mathrm{Ar}^{+}{ }^{2} \mathbf{P}_{3 / 2}$ peak, it's about $11.6 \mathrm{meV}$ ) worse than at 1.0 torr Helium and $180 \mathrm{~mA}$ discharge current $(10.4 \mathrm{meV})$, and at 0.5 torr Helium and $160 \mathrm{~mA}$ discharge current $(9.6 \mathrm{meV})$ the resolution is $\sim 1 \mathrm{meV}$ better than at 1.0 torr and $180 \mathrm{~mA}$. If changes in lamp operation conditions are not correlated with changes in resolution, the lamp needs cleaning and reconditioning.

Cleaning and reconditioning of the lamp can be done by removing the lamp housing from the top of the main chamber first. Second, remove the collimating quartz capillary 
from the lamp body. This must be done by following 'clean room' procedure that requires one to wear surgical gloves to prevent 'oil, grease' contamination of the capillary. Third, remove the discharge capillary from the lamp body, and changing to a new capillary. Fourth, remove the Tantalum linings of the cathode and changing to new tantalum sheet linings. The reverse order should be followed to reassembling the lamp. Before install the lamp back on top of the main chamber, check the electric continuity between the tip of the quartz collimating capillary (it's usually coated with Aquadag graphite) and the lamp body. This will eliminate any possible "charging-up effect" in the quartz capillary.

\section{c. Tuning up of the Electronics: $V_{3}$ and $V_{6}$ Lenses}

With ä clean beam source chamber and main chamber, and a properly functioning lamp, the next step is the time-consuming 'tuning up' of the electronics, especially all the lens voltage settings. From experience, the most sensitive ones are the $V_{3}$ curve and $V_{6}$ voltage settings. It is a good practice to record all the previously used settings before attempting to tune any of those voltages.

The $\mathrm{V}_{3}$ voltages were determined from a series of calibration gases $(\mathrm{Ar}, \mathrm{Kr}, \mathrm{Xe}$, $\left.\mathrm{N}_{2}, \mathrm{O}_{2}, \mathrm{CO}, \mathrm{CO}_{2}\right) .{ }^{1}$ The voltages obtained were fitted to a polynomial function and programmed into the computer. The quality of the curve obtained strongly affects the transmission property of the whole system and the best achievable resolution. It is usually optimized by checking at several different $\mathrm{V}_{\mathrm{k}}$ values using $\mathrm{Ar}, \mathrm{Kr}$, and $\mathrm{Xe}$, adjusting the $V_{3}$ value manually until you get the best resolution and transmission, then reprograming the computer to correct for any changes.

The $V_{6}$ voltage setting is crucial to the highest resolution achievable, since it is the focusing voltage for the 8:1 three-element asymmetric lens. Its value deviates substantially from the theoretically calculated value. There are two 'sharp' focus values: one is a higher voltage usually around $8.000 \pm 4.000$ volts, and the other is a lower voltage usually around $1.000 \pm 0.500 \mathrm{volt}$ (all these voltages were referenced to $V_{k}$, the kinetic energy of the 
photoelectrons). You should only change the $\mathrm{V}$ value 0.1000 volt or less at one time, and check for the resolution at each $V$ setting.

\section{d. Tuning up the $90^{\circ}$ Sector Analyzer and the Hemispherical Analyzer}

The $90^{\circ}$ sector analyzer is also called the prefilter which describes its function very nicely. It not only cuts down the background electrons, but also focuses the electrons that pass through it to the correct position on the following 8:1 three-element asymmetric lens. Its focusing property affects the 8:1 lens very strongly. The $V_{\text {in }}^{1}-V_{\text {out }}^{1}$ value (that is the voltage difference between the inner and outer $90^{\circ}$ sector) is correlated with $V_{3}$ and $V_{6}$ values. After adjusting $V_{3}$ and $V_{6}$ values, it is usually necessary to adjust the $V_{\text {in }}^{1}-V_{\text {out }}^{1}$ value.

Under most circumstances, the values of $V_{\text {in }}^{1}-V_{\text {out }}^{1}$ are very close to their theoretical ones. It should only be adjusted after everything else has been optimized. It should not be adjusted more than $0.050 \mathrm{eV}$ at one time. You should check the resolution and transmission at each setting, and use the setting that gives the best resolution without compromising too much on transmission.

The hemispherical analyzer needs very little tuning. What you need to do is check all the voltage values against the theoretical ones. Since power supplies drift over time, it is advisable that before you start any measurements, all the voltage settings for the hemispherical analyzer be checked and be tuned to their theoretical values (this includes $V_{\text {in }}^{0}, V_{\text {in }}^{0}-V_{\text {out }}^{0}, V_{\text {fte }}(i)(i=1,6)$, here $V_{\text {fte }}(i)$ are the six field termination electrodes). Since the power supply for the hemispherical analyzer is very stable, only very fine tuning needs to be performed, and a sufficiently long time should be allowed for the power supply to be stabilized before any new tuning. From experience, the adjustment step size should be < $2.0 \mathrm{meV}$. You should have at least 5 to 10 minutes between each adjustment.

\section{e. Tuning the Step Size of the Digitizer}

The step size of the digitizer must match the dispersion of the hemispherical 
analyzer as explained in great detail by James E. Pollard. ${ }^{1}$ The dispersion function of the analyzer is a function of not only the pass energy used, but also the surface conditions of the analyzer. The surface conditions of the analyzer are not easily characterized. Its relationship to the dispersion of the analyzer is not known precisely at this writing. Qualitatively speaking, with a fresh molybdenum coating on the hemispherical analyzer surfaces, the dispersion is best at the very beginning. As time goes by, it degrades gradually. Depend on the operating conditions, the degradation rate varies. It is highly recommended that the dispersion of the analyzer be checked before any measurements are made.

This can be done by nunning $\mathrm{a} \sim 20$ torr of Ar beam. Manually adjust $\mathrm{V}_{\mathrm{k}}$ to put the $\mathrm{Ar}^{+}{ }^{2} \mathbf{P}_{1 / 2}$ or ${ }^{2} \mathbf{P}_{3 / 2}$ peak center on the high $\mathrm{V}_{\mathrm{k}}$ side of the detector, and do a normal scan. Increase $\mathrm{V}_{\mathrm{k}}$ value by $\sim 25 \mathrm{meV}$, do another normal scan, and so on. Then calculate the dispersion of the analyzer by this formula $: D=\left(V_{k}\right.$ (fin) $-V_{k}$ (ini) $) /\left(C h_{i n i}-C h_{f i n}\right)$. This gives the dispersion of the analyzer in units of $\mathrm{meV}$ per channel. Then go to the digitizer memory control electronics chassis, adjust the gain for the pass energy chosen, match the digitizer step size with the dispersion of the analyzer. This is done by running the program called DACLD. Choose DAC Channel \# 1 , give initial value 0 , read the $V_{k}(0)$; and final value 500, read $V_{k}(500) . V_{k}(500)=V_{k}(0)+500 \times$ D. This should be matched to better than $\pm 0.1 \mathrm{meV}$ to avoid any loss of resolution during the dithering scan.

\section{Calibration of the MBPES Machine}

The calibration of the MBPES machine was usually carried out every time before an experiment started, and between scans of each experiment. This is done by running noble gases with their ionization potential known precisely, and other molecules with their ionization potential known to be close to the molecules being studied.

One of the major advantages of the MBPES machine over traditional gas-phase PES machines is the longtime stability of its calibration. For experiments running 
noncondensing, or condensing and conducting but non-corrosive gases, the calibration can be very stable. The drift of kinetic energy is less than $0.5 \mathrm{meV}$ per hour. This drift is limited mainly by the stability of the power supply of the lenses and analyzers. For experiments running condensing, nonconducting and corrosive gases, the calibration is less stable. The drift of kinetic energy is about $1 \mathrm{meV}$ per hour This larger drift is caused largely by contact potential effects, which occur when the surfaces surrounding the ionization region are modified by the gases.

The second aspect of the calibration of the MBPES machine concerns the linearity of the kinetic energy scale. This has been shown to be very good. Deviations from linearity scale are less than $1 \mathrm{meV}$ per $2 \mathrm{eV}$ scan, as measured by the energy difference between $\mathrm{N}_{2}^{+} \widetilde{\mathrm{X}}^{2} \Sigma_{\mathrm{g}}^{+}(v=0)$ state and $\widetilde{\mathrm{B}}{ }^{2} \Sigma_{\mathrm{u}}^{+}(v=0)$ state, which is $3.16981 \mathrm{eV}$ from optical emission spectra ${ }^{13}$, and at higher kinetic energy by measuring the difference between $\mathrm{Ar}^{+}$ $(15.75975 \mathrm{eV})$ and $\mathrm{Xe}^{+}(12.13000 \mathrm{eV})$ ionization potentials (these values have been accurately determined from optical spectroscopy ${ }^{14}$ ). The MBPES machine maitains linearity of $\pm 1 \mathrm{meV}$ over this entire energy range. Its fundamental limit, it seems, is the resolution of the 12 bit digital to analog converter which drives the $V_{k}$ steps, and the stability of all the power supplies. For most experiments, this is more than sufficient to assure that the linearity is not going to be the limiting factor.

Table 1 contains the most frequently used calibration gases and their ionization potentials. All electron kinetic energies are based on ionization using the helium I $\alpha$ (548.3440 $\AA^{15} 171129.148 \mathrm{~cm}^{-1}, 1421.21732 \mathrm{eV}$, using 1986 recommended values, $1 \mathrm{eV}$ $=8065.5408 \mathrm{~cm}^{-1},{ }^{16}$ ) radiation line. 


\section{Experimental Setups and Conditions}

In this section, the experimental conditions common to all experiments reported in this dissertation are presented. The setups used to prepare monomer $\mathrm{H}_{2} \mathrm{CO}, \mathrm{D}_{2} \mathrm{CO}$; $\mathrm{H}_{2} \mathrm{CCO}, \mathrm{D}_{2} \mathrm{CCO}$ and their purification are presented with little explanation, since they were well documented in the literature.6,7

\section{Procedures to Prepare Pure Monomer $\mathrm{H}_{2} \mathrm{CO}$ and $\mathrm{D}_{2} \mathrm{CO}$}

The apparatus used to prepare pure monomer $\mathrm{H}_{2} \mathrm{CO}$ and $\mathrm{D}_{2} \mathrm{CO}$ from their polymeric form (paraformaldehyde $99.5 \%$ pure, paraformaldehyde- $\mathrm{d}_{6}$ 99.9\% D-atom, from MSD Isotopes) is shown in Figure 3., following the literature 6 closely with minor modifications. We used a larger diameter $(20 \mathrm{~mm})$ quartz tube made packed-three-U separator (350 mm long) to purify the monomer. The whole apparatus was set up inside a 100 LFT per minute walk-in fume hood.

It's well known that trace amounts of water catalytically polymerizes monomer formaldehyde into its polymer form. This problem was treated by the following procedures. The apparatus was sealed, and the whole system was pump down to $\sim 3 \times 10^{-}$ 6 torr using a $\mathrm{LN}_{2}$-baffled 100 liter-per-second diffusion pump (with DC705 diffusion pump fluid) backed by a two-stage rotary-vane oil-sealed mechanical pump. Then the valve on top of the sample tube was opened to pump off the gases trapped in the sample tube; the tube was heated slightly with a DC704 fluid bath to $100^{\circ} \mathrm{C}$ for $\sim 30$ minutes to drive most of the water out. The system vacuum leveled off at $\sim 1 \times 10^{-5}$ torr. The valve was then closed; the whole system except for the sample tube was baked at $\sim 200^{\circ} \mathrm{C}$ overnight (this would also remove oxygen on the glass surface)). The system vacuum levels off to $\sim 8 \times 10^{-7}$ torr at the end of the bake out.

While the system was still hot, the valve to the sample tube was opened (while pumping all the time). The sample was heated to $70^{\circ} \mathrm{C}$ with the DC704 fluid bath heated by a temperature-controlled electric heating plate. The bottom part of the packed $U$ 
separator was cooled down to $-78^{\circ} \mathrm{C}$ with a dry ice/acetone slush, while the top $1 / 3$ of the packed-three-U separator was kept at $\sim 120^{\circ} \mathrm{C}$ by resistive heating with Nichrome wire wound around it and controlled by a Variac. The collector was then cooled down to $\mathrm{LN}_{2}$ temperature to collect the purified monomer formaldehyde. Any noncondensable gases were pumped away. During the whole preparation process, the vacuum was maintained at $\sim 5 \times 10-6$ torr. The formaldehyde prepared this way was stored at $\mathrm{LN}_{2}$ temperature while pumping until MBPES experiments started.

Essentially the same procedure was followed to prepare pure monomer $\mathrm{D}_{2} \mathrm{CO}$, except at the very beginning. Due to the much higher cost of $\mathrm{D}_{2} \mathrm{CO}$ polymer, the sample was degassed and dehydrated at room temperature (instead of $100^{\circ} \mathrm{C}$ ) for about two hours without heating. The system vacuum was about $5 \times 10^{-6}$ torr after this degassing.

For both $\mathrm{H}_{2} \mathrm{CO}$ and $\mathrm{D}_{2} \mathrm{CO}$, the preparation process took about 48 hours (not including the bakeout time) to get about 20 grams of pure monomer. The monomers prepared this way seemed to be very stable when stored at $\mathrm{LN}_{2}$ temperature, with no sign of polymerization or decomposition.

\section{Procedures to Prepare and Purify $\mathrm{H}_{2} \mathrm{CCO}$ and $\mathrm{D}_{2} \mathrm{CCO}$}

The apparatus used to prepare $\mathrm{H}_{2} \mathrm{CCO}$ and $\mathrm{D}_{2} \mathrm{CCO}$ from pyrolysis of acetic anhydride and acetic anhydride- $\mathrm{d}_{6}$ was similar to the ones used in Prof. C. B. Moore's group with minor modifications, Figure 4. The differences are a longer pyrolysis quartz tube $\sim 25 \mathrm{~cm}$ and a larger cold-finger trap. ${ }^{7}$

The system was first pumped down to a vacuum of $\sim 3 \times 10^{-6}$ torr using the same pumping system as in formaldehyde preparation. Then the sample was degassed using several freeze-thaw cycles. The valve on top of the sample vial was closed after this. The system was then baked out at $\sim 200^{\circ} \mathrm{C}$ overnight to reach a base vacuum $\sim 6 \times 10^{-7}$ torr at the end of the bakeout. 
The quartz pyrolysis tube was heated to $\sim 550^{\circ} \mathrm{C}$ (measured with a thermocouple) with Nichrome wire wound around it and controlled by a Variac. The cold finger trap was cooled down to $-78^{\circ} \mathrm{C}$ with a dry ice/acetone slush. Collector \#1 was cooled down to $\mathrm{LN}_{2}$ temperature to collect the product while collector $\# 2$ was at $\sim 100^{\circ} \mathrm{C}$. Now, the valve to the sample vial was opened. During the whole process, the system vacuum was maintained at $\sim 5 \times 10^{-6}$ torr. After enough ketene had been collected ( $\sim 40$ grams in 48 hours) in collector \#1, the valve between the cold finger and collector \#1 was closed first, the sample vial valve was closed next and the heating tumed off on the pyrolysis tube.

The ketene prepared was then vacuum distilled once from a $\mathrm{LN}_{2} /$ hexane slush (with temperature $\sim-96^{\circ} \mathrm{C}$ ) in collector \#1 to $\mathrm{LN}_{2}$ temperature in collector \#2, and stored in collector \#2 at $\mathrm{LN}_{2}$ temperature under dark with pumping on until the MBPES experiments started. The ketene prepared in this way was very stable; free of $\mathrm{CO}_{2}$, and showed no sign of decomposition when stored at $\mathrm{LN}_{2}$ temperature under dark. $\mathrm{D}_{2} \mathrm{CCO}$ was prepared and stored by exactly the same procedure under the same conditions.

\section{Experimental Conditions for $\mathrm{H}_{2} \mathrm{CO}$ and $\mathrm{D}_{2} \mathrm{CO}$ Photoelectron Spectroscopy}

The Photoelectron spectra of $\mathrm{H}_{2} \mathrm{CO}$ and $\mathrm{D}_{2} \mathrm{CO}$ were taken under similar conditions. The monomer $\mathrm{H}_{2} \mathrm{CO}$ or $\mathrm{D}_{2} \mathrm{CO}$ was kept at dry ice/acetone slush temperature. Its vapor pressures were about 35 torr and 30 torr, respectively, when measured with a Baratron (MKS model 122AA-2000). About 300 torr of ultra-pure He (Matheson $99.9999 \%$ ) was first passed through an $\mathrm{U}$ tube maintained at $\mathrm{LN}_{2}$ temperature to remove trace amount of water, then bubbled through $\mathrm{H}_{2} \mathrm{CO}$ or $\mathrm{D}_{2} \mathrm{CO}$, making the total pressure $\sim 350$ torr. The gas line was first cleaned by heating up to $200^{\circ} \mathrm{C}$ for $\sim 2$ hours with the pump on. Then 300 torr of dry helium was run for $\sim 2$ hours. Immediately after an Ar calibration run, the photoelectron spectra of $\mathrm{H}_{2} \mathrm{CO}$ and $\mathrm{D}_{2} \mathrm{CO}$ were taken. It has been shown that as long as the partial pressure of $\mathrm{H}_{2} \mathrm{CO}$ or $\mathrm{D}_{2} \mathrm{CO}$ was less than 40 torr, no polymerization happens. ${ }^{8}$ 
The photoelectron spectra of $\mathrm{H}_{2} \mathrm{CO}$ and $\mathrm{D}_{2} \mathrm{CO}$ were each taken in four separate scans. Each scan lasted about two hours, and each scan was followed by an Ar calibration scan. The kinetic energy scale shift was less than $1 \mathrm{meV}$ from scan to scan as calibrated with Ar. To enhance statistics. all scans were repeated three times. For each compound, a total of sixteen scan were done.

The mixture of $\mathrm{He} / \mathrm{H}_{2} \mathrm{CO}$ and $\mathrm{He} / \mathrm{D}_{2} \mathrm{CO}$ with total pressure $\sim 350$ torr was expanded through a $70 \mu \mathrm{m}$ converging type molybdenum nozzle, skimmed by a $0.858 \mathrm{~mm}$ diameter $6.4 \mathrm{~mm}$ tall stainless steel skimmer with $D_{n s}=6.4 \mathrm{~mm}$. The beam source chamber pressure was $5 \times 10^{-5}$ Torr. The main chamber pressure was $3 \times 10^{-6}$ torr. The analyzer chamber pressure was at $1.9 \times 10^{-8}$ torr during the scans. The resolution was $10.4 \mathrm{meV}$ FWHM as measured with $\mathrm{Ar}{ }^{2} \mathbf{P}_{1 / 2},{ }^{2} \mathbf{P}_{3 / 2}$ and $\mathrm{Xe}^{2} \mathbf{P}_{1 / 2},{ }^{2} \mathbf{P}_{3 / 2}$. Under these conditions, no polymer or clusters of formaldehyde were found by using the QMS. The rotational temperature was $<10 \mathrm{~K}$ as shown by laser induced fluorescence (LIF) measurements under similar conditions. ${ }^{5}$

\section{Experimental Conditions of $\mathrm{H}_{2} \mathrm{CCO}$ and $\mathrm{D}_{2} \mathrm{CCO}$ Photoelectron Spectroscopy.}

The experimental conditions of $\mathrm{H}_{2} \mathrm{CCO}$ and $\mathrm{D}_{2} \mathrm{CCO}$ were very similar to those used for $\mathrm{H}_{2} \mathrm{CO}$ and $\mathrm{D}_{2} \mathrm{CO}$ with the exception that the samples were kept at Hexane/LN slush temperature $\left(-96^{\circ} \mathrm{C}\right.$, vapor pressure 50 and 45 torr) instead of dry ice temperature ($78{ }^{\circ} \mathrm{C}$, vapor pressure 100 torr) to minimize possible dissociation of ketene. It was later confirmed by photoelectron spectra taken at dry ice/acetone slush temperature that ketene prepared and stored in the procedures described in this section was free of $\mathrm{CO}_{2}$ dissociation products.

The photoelectron spectra of $\mathrm{H}_{2} \mathrm{CCO}$ and $\mathrm{D}_{2} \mathrm{CCO}$ were each taken by five separate scans. Each scan lasted about 2 hours, followed by an Ar calibration scan. The kinetic energy scale shift was $<1 \mathrm{meV}$ from scan to scan as calibrated with Ar. To enhance 
statistics, all scans were repeated three time. Thus for each compound a total of 20 scans were done.

The mixture of $\mathrm{He} / \mathrm{H}_{2} \mathrm{CCO}$ and $\mathrm{He} / \mathrm{D}_{2} \mathrm{CCO}$ with a total pressure $\sim 300$ torr was expanded through a $70 \mu \mathrm{m}$ nozzle and skimmed by a $0.858 \mathrm{~mm}$ diameter $6.4 \mathrm{~mm}$ tall stainless steel skimmer with $D_{n s}=6.4 \mathrm{~mm}$. The beam source chamber pressure was $3 \times 10^{-5}$ torr; the main chamber pressure was $2 \times 10^{-6}$ torr and the analyzer chamber pressure was $1.8 \times 10^{-8}$ torr during the scans. The resolution was $11.6 \mathrm{meV}$ FWHM as measured with $\operatorname{Ar}^{2} \mathbf{P}_{3 / 2}$.

Under these conditions, no dimer or higher clusters of ketene were found by using the QMS. The rotational temperature was estimated to be $<10 \mathrm{~K}$. This was confirmed by LIF experiments carried out under similar conditions. ${ }^{9-12}$ 


\section{References:}

1. James E. Pollard, Ph.D. Thesis, Department of Chemistry, The University of California at Berkeley (1982).

2. Dennis J. Trevor, Ph.D. Thesis, Department of Chemistry, The University of California at Berkeley (1980).

3. Janice E. Reutt, Ph.D. Thesis, Department of Chemistry, The University of Califomia at Berkeley (1986).

4. Laisheng Wang, Ph.D. Thesis, Department of Chemistry, The University of California at Berkeley (1990).

5. W. E. Polik, Ph.D. Thesis, Department of Chemistry, The University of California at Berkeley (1988).

6. R. Spence, W. Wild; J. Chem. Soc., 338 (1935); 506(1935).

7. G.J. Fisher, A.F. MacLean, A.W. Schwizer; J. Org. Chem., 18, 1055(1953)

8. Dr. Roger Van Zee, private communication.

9. I-C Chen, and C.B. Moore, J. Phys. Chem., 24, 263 (1990)

10. I-C Chen, and C.B. Moore, J. Phys. Chem., 94, 269 (1990)

11. E.R. Lovejoy, S.K. Kim, and C.B. Moore, Science, 256, 1541 (1992)

12. I-Chia Chen, W.H. Green, Jr., and C. B. Moore, J. Chem. Phys., 89(1), 314 (1988)

13. A. Lofthus, and P.H. Krupenie, J. Phys. Chem. Ref. Data, 6, 113 (1977)

14. Atomic Energy Levels, Vol. I, II, III, ed. by C. E. Moore, (National Bureau of Standards, Washington D.C., 1958).

15. J. Eland, Photoelectron Spectroscopy, 2nd ed, Butterworths, p32(1984).

16. E. R. Cohen, and B. N. Taylor, The 1986 Adjustınent of the Fundamental Physical Constants, report of the CODATA Task Group on Fundamental Constants, CODATA Bulletin 63, Pergamon, Elmsford, NY(1986). 
Table 1. Calibration Gases \& their Ionization Energies

\begin{tabular}{|c|c|c|c|}
\hline $\begin{array}{l}\text { Calibration } \\
\text { Gas }\end{array}$ & Ionic State & $\begin{array}{l}\text { Electron Kinetic } \\
\text { Energy (eV) }\end{array}$ & $\begin{array}{l}\text { Ionization } \\
\text { Potential (eV) }\end{array}$ \\
\hline $\mathrm{CH}_{3} \mathrm{I}$ & ${ }^{2} \mathbf{E}_{3 / 2}$ & 11.6800 & 9.5380 \\
\hline $\mathrm{CH}_{3} \mathrm{I}$ & ${ }^{2} \mathbf{E}_{1 / 2}$ & 11.053 & 10.1640 \\
\hline $\mathbf{X e}$ & $2 \mathbf{P}_{3 / 2}$ & 9.0880 & 12.1300 \\
\hline $\mathbf{X e}$ & ${ }^{2} \mathbf{P}_{1 / 2}$ & 7.7820 & 13.4360 \\
\hline $\mathbf{K r}$ & ${ }^{2} \mathbf{P}_{3 / 2}$ & 7.2190 & 13.9990 \\
\hline $\mathbf{K r}$ & $2 \mathbf{P}_{1 / 2}$ & 6.5530 & 14.6650 \\
\hline $\mathbf{H g}$ & ${ }^{2} \mathbf{D}_{5 / 2}$ & 6.3780 & 14.8400 \\
\hline $\mathbf{A r}$ & ${ }^{2} \mathbf{P}_{3 / 2}$ & 5.4590 & 15.7598 \\
\hline Ar & ${ }^{2} \mathbf{P}_{1 / 2}$ & 5.2810 & 15.9370 \\
\hline $\mathbf{H g}_{\mathbf{g}}$ & ${ }^{2} \mathbf{D}_{3 / 2}$ & 4.5140 & 16.7040 \\
\hline $\mathbf{N}_{\mathbf{2}}$ & $2 \Sigma_{u}^{+}$ & 2.4670 & 18.7510 \\
\hline $\mathrm{CO}_{2}$ & ${ }^{2} \Sigma_{g}^{+}$ & 1.8236 & 19.3944 \\
\hline $\mathrm{CO}$ & ${ }^{2} \Sigma_{u}^{+}$ & 1.4980 & 19.7200 \\
\hline $\mathbf{O}_{2}$ & ${ }^{2} \Sigma_{g}^{-}$ & 0.9200 & 20.3580 \\
\hline
\end{tabular}


Figure Captions:

Figure 1 Figure 1 is a schematic top view of the apparatus: (1) beam source, (2) beam catcher (not installed), (3) pass energy selector lens, (4) field lens, (5) kinetic energy scan lens, (6) deflectors, (7) $90^{\circ}$ spherical sector preanalyzer, (8) differential pumping wall, (9) 8:1 decelerator lens, (10) hemispherical analyzer, (11) multichannel detector, (12) ion extraction lenses, (13) quadrupole mass filter, (14) differential pumping for various part of the apparatus. The photon source is located above the plane of the drawing. Not shown are two layers of $\mu$-metal magnetic shielding that line the main chamber and surrounding electrostatic lenses, three layers of $\mu$-metal magnetic shielding that line the analyzer chamber, and a grounding plate (with a one inch hole) directly opposite the molecular beam on the far side of the ion optics.

Figure 2 Figure 2 shows the high-resolution electron-energy analyzer, electrostatic lenses, and the multichannel detector in great detail with all the voltage designations, without the $\mu$-metal magnetic shielding in place. F.T.E. stands for Field Termination Electrodes, MCP stands for Microchannel Plates, $X_{1}$ and $X_{2}$ are the Resistive Anode Encode (RAE) outputs.

Figure 3 Figure 3 shows the schematic setups used to prepare monomer formaldehyde and deuterated formaldehyde from paraformaldehyde and $d_{6}$-paraformaldehyde. (1) sample vial, (2) ground glass joint with Teflon sleeve sealing, (3) viton seal high vacuum glass valves, (4) packed-three-U separator, (5) monomer collector, (6) to $\mathrm{LN}_{2}$ trapped diffusion pump. Not shown are the Nichrome wires wound around the connection from the sample vial to and the top $1 / 3$ of the packed-three-U separator, and the 
Dewars for the packed-three-U separator (dry ice/acetone slush temperature) and the monomer collector ( $\mathrm{LN}_{2}$ temperature). For operating conditions, see main text.

Figure 4 Figure 4 shows the schematic setups used to prepare and purify ketene and deuterated ketene from acetic anhydride and acetic anhydride- $d_{6}$. (1) sample vial, (2) ground glass joint with Teflon sleeve sealing, (3) viton seal high vacuum glass valve, (4) quartz pyrolysis tube, (5) cold finger to collect acetic acid, the by-product, (6) collector \# 1, (7) collector \# 2, (8) Nichrome heating wire, (9) to $\mathrm{LN}_{2}$ trapped diffusion pump. Not shown are Dewars used to maintain $\mathrm{LN}_{2}$ temperature during preparation for collectors \# 1 \& 2 , and a thermocouple used to measure the pyrolysis temperature. For operation conditions, see main text. 


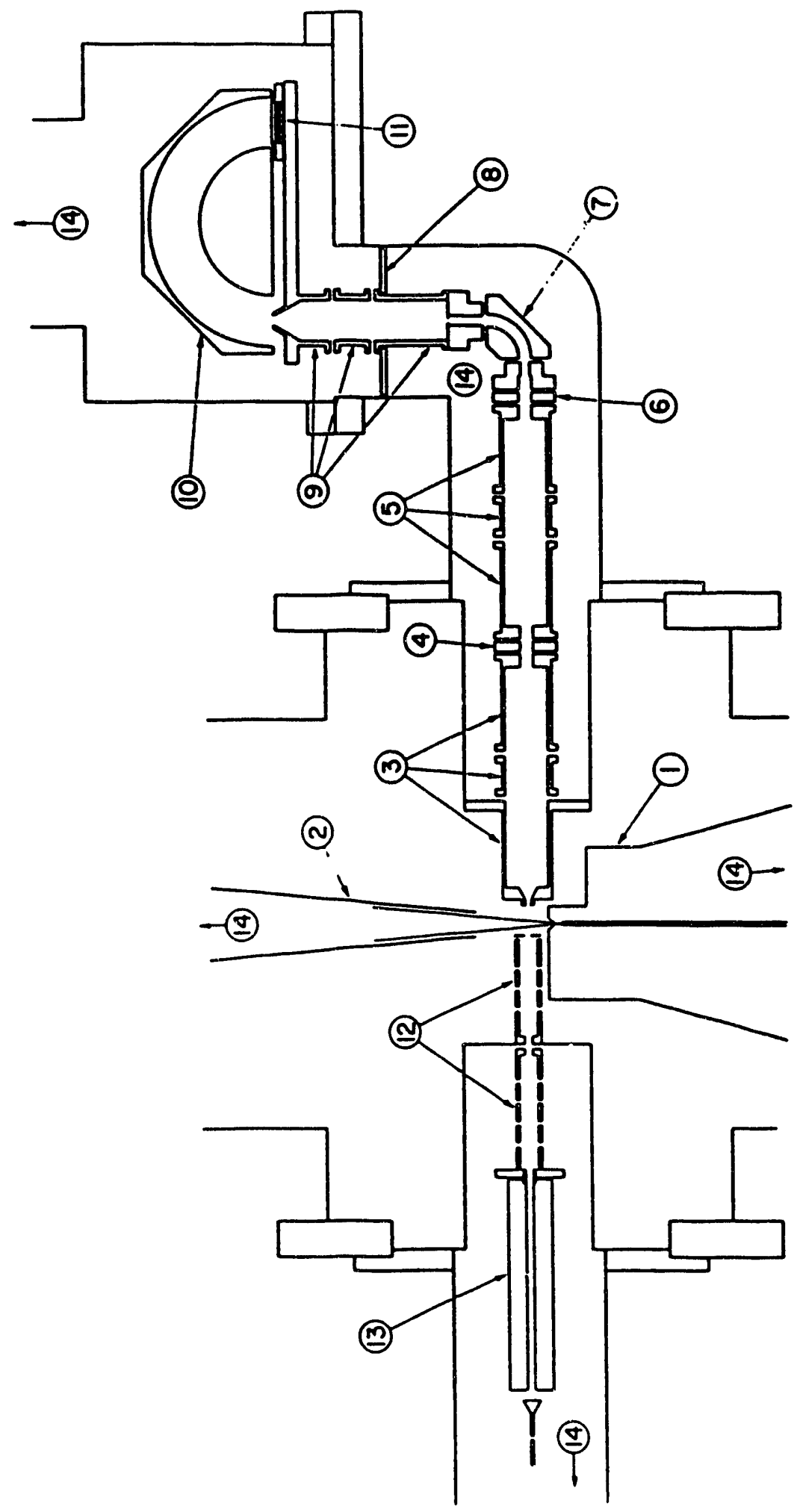

氙 


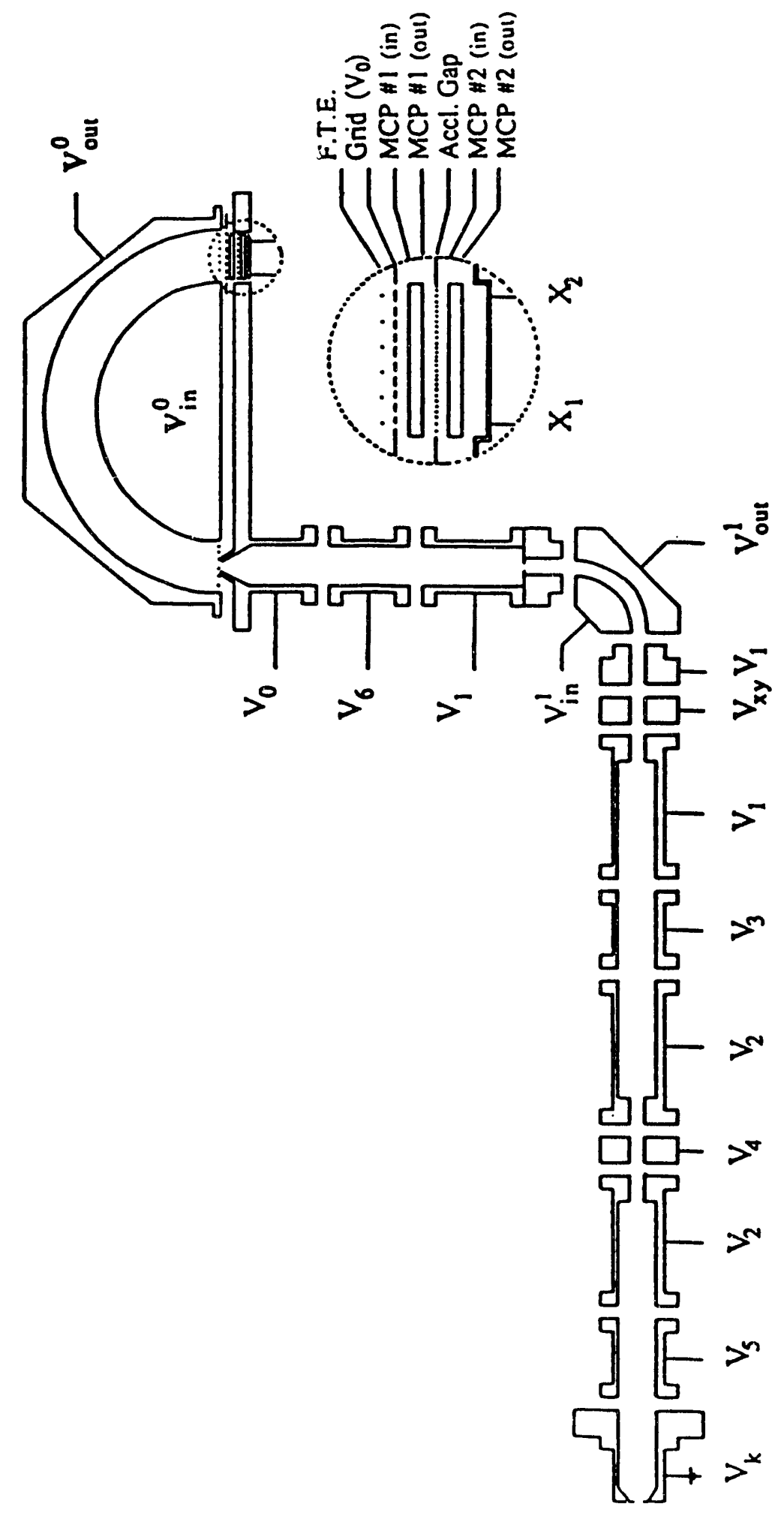

瓷 


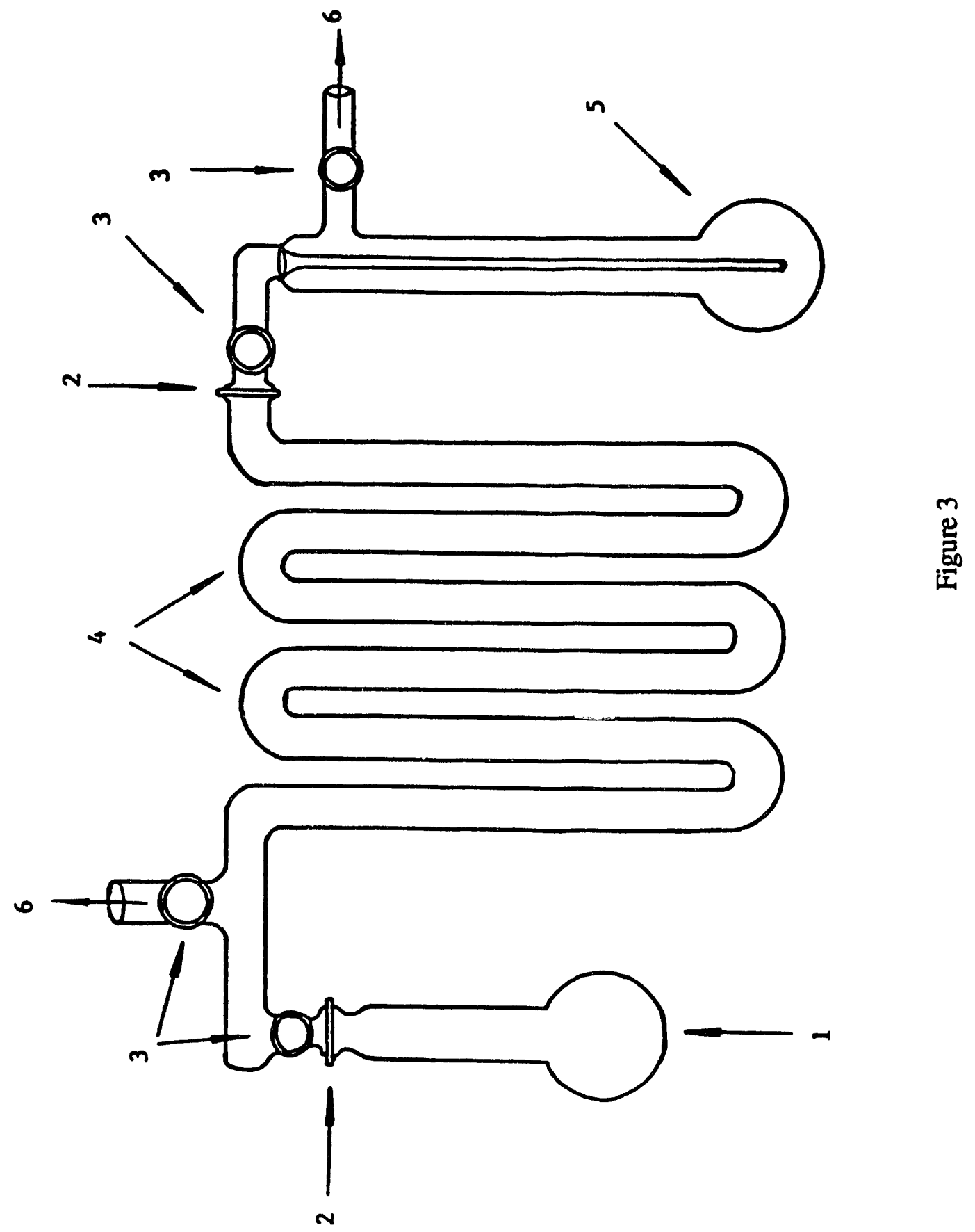




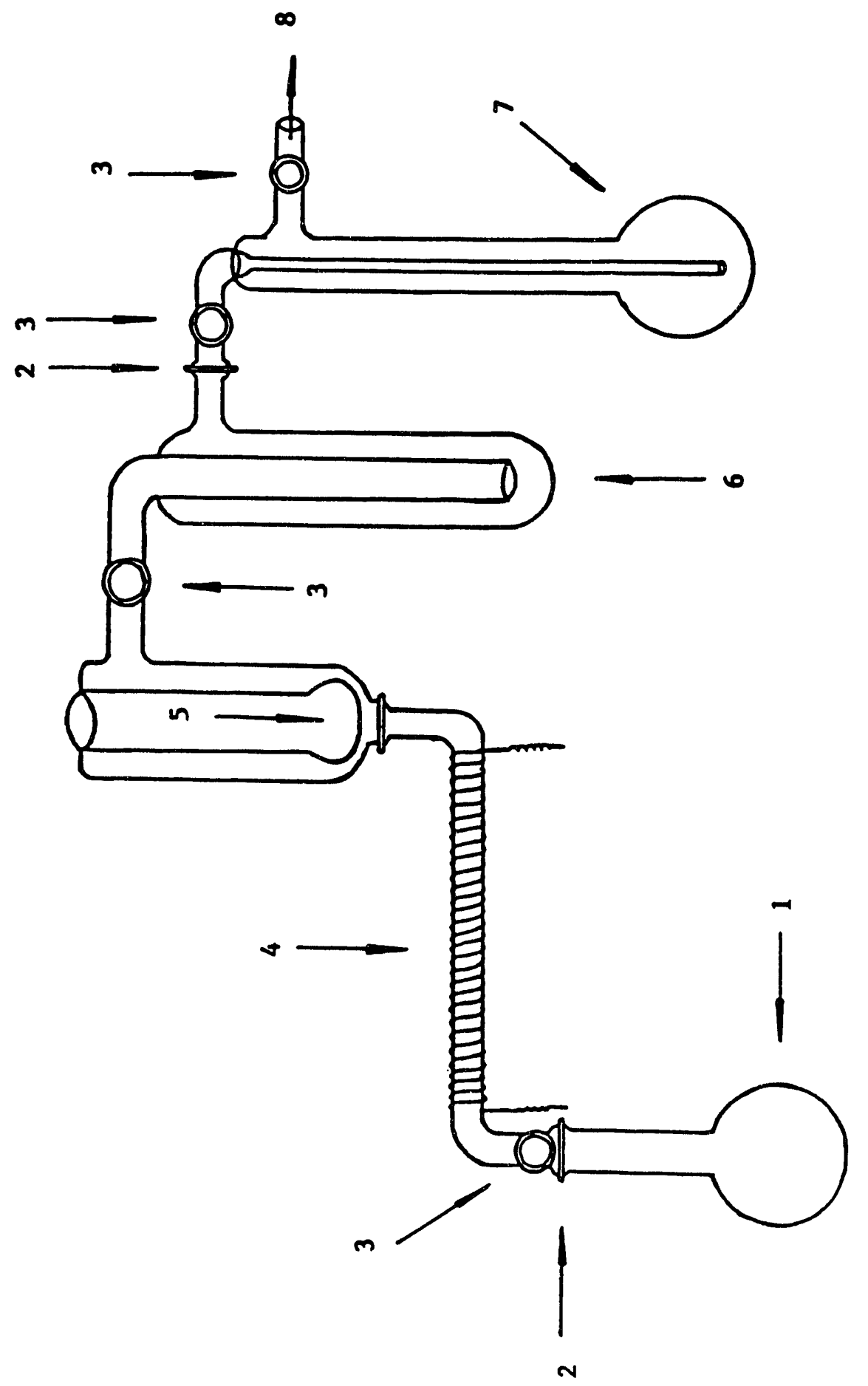

8
0
0
0 


\section{Chapter Three High Resolution Photoelectron Spectroscopy and Femtosecond Intramolecular Dynamics of $\mathrm{H}_{2} \mathrm{CO}^{+}$and $\mathrm{D}_{2} \mathrm{CO}^{+}$}

\section{A. Introduction}

The photoelectron spectra of $\mathrm{H}_{2} \mathrm{CO}$ and $\mathrm{D}_{2} \mathrm{CO}$ have been the subject of extensive theoretical and experiment studies. ${ }^{1-10}$ Most of the theoretical studies have based on the earlier experimental studies by Turner et al. ${ }^{1}$, and by Baker et al. ${ }^{2}$ The assignment of the four outer nost valence states' adiabatic ionization potentials by Tumer et al. has been the subject of some theoretical calculations, especially on the ordering of the third and fourth ionic states. The vibrational fine structures observed in these low-resolution works have been studied extensively by theoretical calculations. 3-10 Although the vibrational fine structures seemed well resolved and relatively simple in most of the electronic states, their interpretation, however, has caused considerable difficulties. $1,2,11,12$ Cederbaum $^{8}$ and coworkers used a many-body approach to the vibrational structures in formaldehyde, and cautioned that the vibrational coupling could be very different for different isotopic species. There were some disagreements between the theoretical and experimental results regarding the vibrational assignments. 13

The stability and the decay pathways, and the dynamics of the formaldehyde ions formed by photoionization in various ionic states have also been studied both theoretically and experimentally. ${ }^{14-17}$ In a photoionization study by Guyon et al., 14 they suggested that the decay of the first excited state $\widetilde{\mathrm{A}}^{2} \mathrm{~B}_{1}$ state produces the $\mathrm{HCO}^{+}$and $\mathrm{CO}^{+}$fragments. By using the correlation diagrams, they ascribed the production of the $\mathrm{CO}^{+}$ion to the electronic predissociation of the $\tilde{A}^{2} B_{1}$ state by an upper repulsive ${ }^{2} \mathrm{~A}_{1}$ state leading to the $\mathrm{CO}^{+}\left({ }^{2} \Sigma^{+}\right)+\mathrm{H}_{2}\left({ }^{1} \Sigma_{\mathrm{g}}^{+}\right)$asymptote at $14.1 \mathrm{eV}$. They proposed that the $\tilde{\mathrm{A}}^{2} \mathrm{~B}_{1}$ state was predissociated by the ground $\widetilde{\mathrm{X}}{ }^{2} \mathrm{~B}_{2}$ State, whose lowest asymptote leads to $\mathrm{HCO}^{+}\left({ }^{1} \Sigma^{+}\right)+$ 
H (2S) fragments. An $a b$ initio calculation study by Pires et al., 18 gave some justification and explanations about the exact decay mechanism in the experimental observations by invoking a particular type of intersections between the potential energy surfaces: conical intersections. The fragmentation of the $\mathrm{H}_{2} \mathrm{CO}+$ in $\widetilde{\mathrm{A}}{ }^{2} \mathrm{~B}_{1}$ state has also been studied by coincidence experiments $15-19$. It was determined that at $v=6$, the formaldehyde ion in the $\tilde{\mathrm{A}}^{2} \mathrm{~B}_{1}$ state starts to fragment and yields $\mathrm{CO}^{+}+\mathrm{H}_{2}$, and at $\mathrm{v}=7$, deuterated formaldehyde ion in the $\tilde{A}^{2} \mathrm{~B}_{1}$ starts to fragment and yields $\mathrm{CO}^{+}+\mathrm{D}_{2} .{ }^{15}$

In this work, we report the high resolution (FWHM $11 \mathrm{meV}$ ) helium I $\alpha$ photoelectron spectra of $\mathrm{H}_{2} \mathrm{CO}$ and $\mathrm{D}_{2} \mathrm{CO}$. Improved resolution and effective cooling of the sample by the supersonic expansion enabled us to determine the adiabatic ionization potentials for the states ionizable by the helium I $\alpha$ line $(21.21804 \mathrm{eV})$ to a much higher accuracy, and to give new interpretations on the vibrational structures observed in all ionic states. The results agree fairly well with available theoretical calculations. The observations that in the ground and first excited states of the $\mathrm{H}_{2} \mathrm{CO}^{+}$and $\mathrm{D}_{2} \mathrm{CO}^{+}$odd quanta of non-totally symmetric mode $\left(v_{4}\right)$ was excited, showed the possibility that the molecular ions in these electronic states might have non-planar equilibrium geometry. This was not discussed in any of the previously experimental and theoretical works. This was argued qualitatively by using the method first developed by R.S. Mulliken 20 and later by A.D. Walsh, 21,22 known as the Walsh diagram. Using the autocorrelation function formalism first discussed by Heller ${ }^{23-26}$ for electronic photoabsorption and emission, and late by Lorquet et al. ${ }^{27-29}$, and by Ruscit $28 \mathrm{~b}$ for photoelectron spectroscopy, modified by Pollard et al. ${ }^{30}$ and Reutt et al., 31,32 the femtosecond ultra fast intramolecular dynamics of the formaldehyde cation in its various ionic states is discussed.

The details of the experiments are described in section B of this chapter. The vibronic coupling and isotope effects on vibronic couplings are discussed briefly in section 
C. A description and the method for calculating the autocorrelation functions are presented in section D. Spectroscopic results and the dynamic interpretations based on the vibrational autocorrelation functions are discussed for each of the electronic states of $\mathrm{H}_{2} \mathrm{CO}^{+}$and $\mathrm{D}_{2} \mathrm{CO}^{+}$in section $\mathrm{E}$. The major conclusions are summarized in section $\mathrm{F}$.

\section{B . Experiment}

The molecular beam photoelectron spectrometer used for this study has been described in great detail by Pollard et al. ${ }^{34}$ and by Reutt et al. ${ }^{35}$ Briefly, it consists of a differentially pumped supersonic molecular beam source for the introduction of the sample into the main chamber where ionization takes place; a differentially pumped windowless helium discharge lamp optimized to produce only the helium I $\alpha$ resonance line with minor contamination from higher resonance lines; a differentially pumped quadrupole mass spectrometer (QMS) to characterize the beam composition; a differentially pumped high resolution electron energy analyzer consisting of a $90^{\circ}$ spherical sector prefilter, a $180^{\circ}$ hemispherical analyzer equipped with a multichannel detector, and associated electron optics; and a dedicated microcomputer for data acquisition and control. The electrons are collected at a $90^{\circ}$ angle with respect to the incident photon beam and the supersonic molecular, beam and the intensity is uncorrected for angular distribution effects. Formaldehyde $\beta$ values for helium I $\alpha$ photons are not available, although there are angleresolved photoelectron studies ${ }^{33}$ on formaldehyde in the photon energy range $10-30 \mathrm{eV}$ using synchrotron radiation, which gave the $B$ values for the $\widetilde{X}^{2} B_{2}, \widetilde{A}{ }^{2} B_{1}, \widetilde{C}^{2} A_{1}$, and $\widetilde{D}$ ${ }^{2} \mathrm{~B}_{2}$ states at photon energy around $21 \mathrm{eV}: 0.5,0.5,0.2,0.3$, respectively.

Approximately 300 torr of ultra high purity helium (99.9999\%, Matheson) was first passed through a $U$ tube maintained at $\mathrm{LN}_{2}$ to remove trace amount of water in the carrier gas line, then bubbled through liquid monomer $\mathrm{H}_{2} \mathrm{CO}$ or $\mathrm{D}_{2} \mathrm{CO}$ maintained at acetone/dry ice slush temperature $\left(-78^{\circ} \mathrm{C}\right)$ with vapor pressures about 35 torr and 30 Torr 
as measured with an MKS model 122AA-2000 Baratron. The monomer $\mathrm{H}_{2} \mathrm{CO}$ or $\mathrm{D}_{2} \mathrm{CO}$ was prepared following a literature procedure 36 by thermocracking paraformaldehyde and fully deuterated paraformaldehyde (MSD Isotopes, $99.9 \%$ D-atom) at approximately 120 ${ }^{\circ} \mathrm{C}$, fractioning (at $-78^{\circ} \mathrm{C}$ ), trapping the monomer (at $\mathrm{LN}_{2}$ temperature), and storing under dark at $\mathrm{LN}_{2}$ temperature before use.

The $\mathrm{He} / \mathrm{H}_{2} \mathrm{CO}$ or $\mathrm{He} / \mathrm{D}_{2} \mathrm{CO}$ mixture with a total pressure of approximately 350 Torr was expanded through a $70 \mu \mathrm{m}$ diameter converging molybdenum nozzle held at room temperature, skimmed by a $0.858 \mathrm{~mm}$ diameter, $6.4 \mathrm{~mm}$ tall conical stainless steel skimmer, $D_{n s}=6.4 \mathrm{~mm}$. The beam source chamber pressure was $6 \times 10^{-5}$ torr, and the main chamber pressure was $3 \times 10^{-6}$ torr during the experimental measurements. All gas inlet lines were minimized and extensively baked $\left(200^{\circ} \mathrm{C}\right)$ under vacuum $\left(10^{-7}\right.$ torr) to remove trace amounts of water which is known to catalytically polymerize formaldehyde. Beam compositions were checked by the quadrupole mass spectrometer. No polymers of $\mathrm{H}_{2} \mathrm{CO}$ or $\mathrm{D}_{2} \mathrm{CO}$ were found under these experimental conditions. The rotational temperature in the beams was estimated to be $<10 \mathrm{~K}$ as shown by LIF measurements under similar corditions. ${ }^{37}$

The complete photoelectron spectrum of each isotopic species was obtained as four sequential scans of the electron kinetic energy. Each scan was preceded and immediately followed by an argon calibration scan. To enhance statistics, four complete spectra of $\mathrm{H}_{2} \mathrm{CO}$ were scanned and summed. Thus the reported photoelectron bands each represent the summation of four scans. Individual scans were made within a time period of less than two hours. The presented $\mathrm{D}_{2} \mathrm{CO}$ photoelectron bands were the summation of four such scans as well. Restricting the length of each scan limits the total drift in the electron kinetic energy scale to $\leq 1 \mathrm{meV}$. The linearity of the kinetic energy scale was determined by obtaining the $N_{2}^{+}$photoelectron spectrum and comparing the $N_{2}^{+} \widetilde{X}^{2} \Sigma_{g}^{+} V=0$ and $\widetilde{B}{ }^{2} \Sigma_{u}^{+} V=0$ splitting with the accurate literature value of $3.16981 \mathrm{eV}$ available from $\mathrm{N}_{2}^{+}$optical emission 
spectroscopy. ${ }^{38}$ At higher kinetic energies, the linearity of the energy scale was checked by the photoelectron spectra of argon and xenon. The ionization potential of these rare gases (I.P. Ar ${ }^{2} \mathrm{P}_{3 / 2}=15.75975 \mathrm{eV}$, and $\mathrm{Xe}^{2} \mathrm{P}_{3 / 2}=12.13000 \mathrm{eV}$ ) have been precisely determined from optical spectroscopy. ${ }^{39}$ The linearity of the molecular beam spectrometer is within $\pm 1.0 \mathrm{meV}$ over this entire energy range. The combination of the drift and the linearity of the energy scale errors limits the accuracy at which the absolute ionization potential may be reported to $\pm 3.0 \mathrm{meV}$. Other spectroscopic constants, however, are obtained as line splittings and may be reported to a much higher accuracy of $\pm 0.5 \mathrm{meV}$.

\section{Vibronic Coupling and Isotope Effects on Vibronic Coupling}

In photoelectron spectroscopy, the vibronic coupling effect, that is the interaction of the electronic and nuclear (vibrational) motion, is often invoked to explain the observations that there are vibrational progressions in modes that are not allowed by the selection rules based on the Born-Oppenheimer approximation; 40 that the observed vibrational frequencies are very low; and that odd quanta of non totally symmetric vibrations get excited. There are other couplings and resonance effects that can also cause similar effects, like vibrational-rotation coupling, rotation-electronic couplings and Fermi resonances. In general, these effects are much smaller than the vibronic coupling effect and are much easier to identify.

The vibronic coupling effects are usually treated as perturbations to the BornOppenheimer approximation, that is the adiabatic, Hamiltonian $\mathscr{K}_{0 .}{ }^{41}$ We can expand the molecular Hamiltonian $\mathscr{X}(\mathrm{q} ; \mathrm{Q})$, which is a function of the electron $\mathrm{q}$ and the nuclear $\mathrm{Q}$ coordinates, with a Taylor series in the normal coordinates of vibrational $Q$ near the equilibrium configuration $\mathrm{Q}_{0}$ :

$$
\mathscr{H}=\mathscr{H}(\mathrm{q} ; \mathrm{Q})_{\mathrm{Q}_{0}}+\sum_{\mathrm{r}=1}^{\infty}\left|\frac{1}{\mathrm{r} !} \frac{\partial^{\mathrm{r}} \mathscr{H}(\mathrm{q} ; \mathrm{Q})}{\partial \mathrm{Q}^{\mathrm{r}}}\right|_{\mathrm{Q}_{0}} \mathrm{Q}^{\mathrm{r}}+\cdots
$$


The first term in equation $(3.1), \mathscr{H}(\mathrm{q} ; \mathrm{Q}) \mathrm{Q}_{0}$, is called the zero-order Hamiltonian, that is the Born-Oppenheimer adiabatic Hamiltonian, and is usually expressed as $\mathscr{X}_{0}$. Applying the total Hamiltonian $\mathscr{H}$ to the molecular wave function $\Psi$ yields:

$$
\langle\Psi|\mathscr{H}| \Psi\rangle=\mathrm{E}_{0}+\mathrm{E}_{1}+\mathrm{E}_{2}+\ldots
$$

Where $E_{0}$ is the non-perturbed energy, and $E_{1}, E_{2}$ are the perturbation energies resulting from the linear and quadratic expansion terms in equation (3.1) respectively. The coupling of two wave functions $\Psi_{\mathrm{k}}$ and $\Psi_{\mathrm{j}}$ can be expressed according to the matrix elements:

$$
\left\langle\Psi_{\mathrm{k}}|\mathscr{H}| \Psi_{\mathrm{j}}\right\rangle
$$

which can be expanded with the Hamiltonian $\mathscr{X}$ of equation (3.1),

$$
\begin{aligned}
& \left\langle\Psi_{\mathrm{k}}|\mathscr{H}| \Psi_{\mathrm{j}}\right\rangle=\left\langle\Psi_{\mathrm{k}}\left|\mathscr{H}_{0}\right| \Psi_{\mathrm{j}}\right\rangle+\sum_{\mathrm{r}} \mathrm{Q}_{\mathrm{r}}\left\langle\Psi_{\mathrm{k}}\left|\frac{\partial \mathscr{H}}{\partial \mathrm{Q}_{\mathrm{r}}}\right| \Psi_{\mathrm{j}}\right\rangle+ \\
& +\frac{1}{2 !} \sum_{\mathrm{r}} \mathrm{Q}_{\mathrm{r}}^{2}\left\langle\Psi_{\mathrm{k}}\left|\frac{\partial^{2} \mathscr{X}}{\partial^{2} \mathrm{Q}_{\mathrm{r}}^{2}}\right| \Psi_{\mathrm{j}}\right\rangle+\frac{1}{2 !} \sum_{\mathrm{rs}} \mathrm{Q}_{\mathrm{r}} \mathrm{Q}_{\mathrm{s}}\left\langle\Psi_{\mathrm{k}}\left|\frac{\partial^{2} \mathscr{H}}{\partial \mathrm{Q}_{\mathrm{r}} \partial \mathrm{Q}_{\mathrm{s}}}\right| \Psi_{\mathrm{j}}\right\rangle+\ldots
\end{aligned}
$$

The first term in equation (3.4) is always zero by the orthogonality relationship. Thus the necessary condition for the vibronic coupling effects to perturb the electronic states involved is that at least one term in the equation (3.4) has a nonzero value that is not too small compared with the zero-order electronic states' energy separations.

The total Hamiltonian $\mathscr{H}$ of equation (3.1) always possesses the full symmetry $\left(\Gamma_{1}\right)$ of the molecular point group. This is also true for $\mathscr{H}_{0}$, the Hamiltonian for the zerothorder unperturbed molecule. For the coupling operators in equation (3.4): $\partial \mathscr{H} / \partial \mathrm{Q}_{\mathrm{r}}$ transforms as the irreducible representation of the normal coordinate $\mathrm{Q}_{r}, \Gamma\left(\mathrm{Q}_{\mathrm{r}}\right) ; \partial^{2} \mathscr{\mathcal { K }} / \partial^{2} \mathrm{Q}_{r}$ 
always has at least one component that transforms as $\Gamma_{1}$, the full symmetry of the molecular point group; and $\partial 2 \mathcal{H} / \partial \mathrm{Q}_{r} \partial \mathrm{Q}_{\mathrm{s}}$ transforms as $\Gamma\left(\mathrm{Q}_{\mathrm{r}}\right) \otimes \Gamma\left(\mathrm{Q}_{\mathrm{s}}\right)$. The linear coupling integral in equation (3.4) will be finite if the product of the representations of all species in the integral contains the totally symmetric representation, $\Gamma_{1}$, that is:

$$
\Gamma\left(\Psi_{k}\right) \otimes \Gamma\left(\Psi_{j}\right) \otimes \Gamma\left(\partial \mathscr{H} / \partial Q_{r}\right) \subset \Gamma_{1}
$$

This will enable the $Q_{\mathrm{r}}$ normal vibrational mode to couple and mix the $\Psi_{\mathrm{k}}$ and $\Psi_{\mathrm{j}}$ electronic states. If $\Psi_{\mathbf{k}}$ and $\Psi_{j}$ are degenerated states, then $Q_{r}$ is the asymmetric vibrational mode that will remove the degeneracy. We can discuss the two quadratic terms in equation (3.4) similarly. The linear terms are usually much larger than the quadratic terms, and the quadratic terms are normally neglected when the linear terms are nonzero. In a polyatomic molecule, there could be more than one normal mode $Q_{r}$ that satisfy the condition of equation (3.5). Those normal modes that satisfy equation (3.5) are called vibronically active modes. When there are more than one modt that are vibronically active, multimode vibronic coupling effects will occur In general, these are more complicated to deal with. Multimode vibronic coupling effects and their influence on spectroscopy have been reviewed in detail by Köppel et al. 42

It can be seen from equation (3.4) that both the linear and quadratic vibronic couplings depend on the normal coordinates that are mass-dependent. It is expected that the couplings will be different for isotope-substituted compounds in the same electronic state. One important consequence of this effect is that it is possible for different vibrational modes to be excited to a different extent for isotope-substituted compounds. Theoretical calculations have shown that these effects appear in the isotope-substituted compounds. ${ }^{36}$ The $\mathrm{H}_{2} \mathrm{CO}^{+}$and $\mathrm{D}_{2} \mathrm{CO}^{+}$photoelectron spectra in their first and second excited states do show this kind of behavior, and they will be discussed further in section $\mathrm{E}$ of this chapter. 


\section{The Correlation Function}

The autocorrelation function formalism description of the intramolecular dynamic process gives complementary information to time-dependent measurements. Lorquet et al. 27-29 first demonstrated how this can be done for photoelectron bands using the formalism of Heller. ${ }^{23-26}$ A variation on the method of Lorquet et al. was used by Pollard et al., 30 and by Reutt et al. 31,02 to study the dynamic characteristics of intramolecular processes using supersonic molecular beam photoelectron spectroscopy. Ruscic ${ }^{28 b}$ has also given the analytical form of the vibrational autocorrelation function for photoelectron bands under the harmonic approximation for vibrational motions.

Very recently, Remacle et al. ${ }^{29}$ have suggested that there exists a relationship between the vibrational autocorrelation function $\mathbf{C}(\mathrm{t})$ and the population decay function $\mathbf{P}(\mathrm{t})$ of a particular electronic state. They defined an average population decay curve $\mathbf{P}_{\mathrm{av}}(t)$ valid in a particular time period, and $C_{a v}(t)$, the average correlation function, obtained from $C(t)$. It was pointed out by the authors that for a specific excitation, the exact initial rate of decay of $\mathbf{P}_{\mathrm{av}}(\mathrm{t})$ (valid up to the dephasing time $T_{1}$ ) is equal to the initial rate of decay of $|\mathbf{C}(\mathrm{t})|^{2}$, and the subsequent time evolution of $\mathbf{P}_{\mathrm{av}}(\mathrm{t})$ can be obtained by averaging $|C(t)|^{2}$ over its oscillation to give $\left|C_{a v}(t)\right|^{2}$. To a good approximation, the average population decay curve $\mathbf{P}_{\mathrm{av}}(\mathrm{t})$ can be obtained by multiplying $\left|\mathrm{C}_{\mathrm{av}}(\mathrm{t})\right|^{2}$ with an appropriate constant; i.e., the slopes of the two curves are the same.

The autocorrelation function can be expressed as

$$
C(t)=|\langle\phi(0) \mid \phi(t)\rangle| \text {. }
$$

Here $\phi(0)$ is the initial nuclear wavefunction, and $\phi(t)$ is the time evolution of this wavefunction on the excited state potential energy surface. $C(t)$ represents the probability amplitude that at time $t$ the system remains in the initially prepared state. It must be noted 
that the correlation function is the measure of the time evolution of the initial wave packet on the excited state potential energy surface and not a measure of the excited state population. But as mentioned in the preceding paragraph, the initial decay rate of $\mathbf{C}(\mathrm{t})$, through $|C(t)|^{2}$, is the same as the average population $\mathbf{P}_{\text {av }}(t)$ initial decay rate. It is dominated by the dephasing of the initial wave packet due to the different shape of the excited-state potential energy surface. Only at times greater than a vibrational period will radiationless decay processes appear in the correlation function time evolution. The derivation of the autocorrelation function $\mathrm{C}(\mathrm{t})$ from photoelectron spectroscopy experimental data has been reported; only the principal points will be outlined here. The form of photoionization cross section under the strict Franck-Condon approximation 40 can be expressed as

$$
\left.\sigma(\mathrm{E}) \propto|| \mathrm{M}_{\mathrm{el}}(\mathrm{Q}, \mathrm{E})\right|^{2}\left|\left\langle\Psi^{\prime \prime} \mid \Psi^{\prime}\right\rangle\right|^{2} \mid
$$

Here $\mathbf{M}_{\mathrm{el}}(\mathrm{Q}, \mathrm{E})$ is the pure electronic transition moment, a function of the nuclear coordinates $\mathrm{Q}$ and electron kinetic energy $\mathrm{E}$, and $\Psi^{\prime \prime}$ and $\Psi^{\prime}$ are the initial- and final-state vibrational wave functions. The electronic transition moment varies slowly over the photoelectron band. In lieu of any arbitrary approximation, a constant value was used in calculating the correlation functions reported here.

Applying the completeness on a set of eigenstate $\Psi^{\prime \prime}$ of the molecular Hamiltonian and invoking the analytical form of the Dirac $\delta$ function, it has been shown ${ }^{28 a}$ that the cross section of photoionization becomes

$$
\sigma(\mathrm{E}) \propto \frac{1}{2 \pi} \int_{-\infty}^{+\infty} \mathrm{e}^{\mathrm{iE} t / \hbar}\left\langle\Psi^{\prime \prime}\left|\mathrm{e}^{-\mathrm{i} \mathscr{X} t / \hbar}\right| \Psi^{\prime \prime}\right\rangle d t
$$


Here $\mathscr{X}$ is the molecular Hamiltonian, $\Psi^{\prime \prime}$ is identified as the initial nuclear wave function $\phi(0)$, and $\mathrm{e}^{-\mathrm{i} \mathscr{t} / \hbar}\left|\Psi^{\prime \prime}\right\rangle$ is the nuclear wave function at time $t: \phi(t)$. This gives

$$
\sigma(E) \propto \frac{1}{2 \pi} \int_{-\infty}^{+\infty} e^{i E t / \hbar}\langle\phi(0) \mid \phi(t)\rangle d t
$$

and the correlation function can be obtained by a Fourier transformation of the cross section

$$
C(t)=|\langle\phi(0) \mid \phi(t)\rangle| \propto \frac{1}{2 \pi} \int_{-\infty}^{+\infty} \sigma(E) e^{-i E t / \hbar} d t
$$

It can be seen from this equation that the correlation function can be obtained from the Fourier transform of the photoelectron partial cross section $\sigma(E)$. This can be accomplished by deconvoluting the instrument response function $\mathrm{I}(\mathrm{E})_{\text {ir }}$, which we determine from the photoelectron spectrum of a rare gas at a kinetic energy comparable to the band of interest, from the quantity $I(E)$, intensity vs. energy, which we measure in photoelectron spectroscopy. The contributions of the finite rotational temperature in a supersonic molecular beam experiment (typically $\sim 5-10$ Kelvin) and the rotational excitations contribution to the correlation function were removed by convoluting $I(E)_{i r}$ with a gaussian function $\mathbf{I}(\mathrm{E})_{\mathrm{g}}$ to generate a rotationally broadened instrument response function. The width of the gaussian function was chosen from the average rotational constant , $\mathbf{B}_{\mathrm{avg}}$, of the molecular ion for the particular electronic state (if available), the finite rotational temperature, and the rotational selection rules for photoionization. Ruscic has taken a different approach to account for all the corrections. However, both methods give essentially the same results in the time window of interest. 
The procedure for calculating the correlation function of $\widetilde{\mathrm{X}}, \widetilde{\mathrm{A}}, \widetilde{\mathrm{B}}$, and $\widetilde{\mathrm{C}}$ states of formaldehyde and $d_{2}$-formaldehyde is the following. First, the band of interest was isolated (in the case of B and C states, they were digitally separated to remove the overlapping of these two bands), and the empirically determined background, plus any constant background, was then removed. The resulting band was then normalized (area = 1) and Fourier transformed using a discrete FFT algorithm. 43 The instrument response function was similarly normalized and convoluted with a Gaussian of $6.5 \mathrm{meV}$ (FWHM). The resulting function was Fourier transformed and divided into that of the data. Finally, the modulus of the previous result was calculated, which gave the correlation function. The procedure can be summarized by the following equation:

$$
\begin{aligned}
& C^{v i b}(t)=|\langle\phi(0) \mid \phi(t)\rangle|=\left|\int_{-\infty}^{+\infty} I(E) e^{-i E t / \hbar} d E\right| \div \\
& \left|\int_{-\infty}^{+\infty} I(E)_{\text {ir }} e^{-i E t / \hbar} d E X \int_{-\infty}^{+\infty} I(E)_{g} e^{-i E t / \hbar} d E\right|
\end{aligned}
$$

Then $|C(t)|^{2}$ was calculated, and $\left|C_{a v}(t)\right|^{2}$ was evaluated by fit $|C(t)|^{2}$ with an exponential curve of the form $\mathrm{Ae}^{-i k t}$ for both the initial drop and the subsequent decaying after the first 'vibrational' period.

\section{E. Results and Discussions}

The full spectra of $\mathrm{H}_{2} \mathrm{CO}$ and $\mathrm{D}_{2} \mathrm{CO}$ obtained by combining four separate scans with a resolution of $11 \mathrm{meV}$ FWHM (as measured with Ar) are shown in Figure 1, and Figure 2, respectively. Table I summarizes the measured spectroscopic constants together with results reported in the literature. 47.48 


\section{First Band, $\tilde{\mathrm{X}} \mathbf{1}^{2} \mathrm{~B}_{2}$ state}

The ground states of both $\mathrm{H}_{2} \mathrm{CO}^{+}$and $\mathrm{D}_{2} \mathrm{CO}^{+}$have well-resolved vibrational fine structures, as shown separately in Figure 3 and Figure 4. Observed spectroscopic results are presented in Table II. In the present work, effective rotationally cooling enables all of the observed vibrational levels to be determined with improved accuracy and the mean energies of these transitions were determined by the following least-squares fitting procedure. The adiabatic transitions (or the most intense feature) were first determined by fitting these features to Gaussians. The adiabatic peaks were then isolated and used as empirical functions to fit the successive vibrational levels. This procedure allows the values of peak splittings to be determined to an accuracy of $\leq \pm 0.0005 \mathrm{eV}$. The mean transition energies located through this procedure are listed for each of the bands observed. The vibrational levels for each normal mode were then least-squares fitted to the standard energy level expression of a Morse oscillator $46 \mathrm{a}, 46 \mathrm{~b}$

$$
\mathbf{G}_{i}^{0}(v)=\omega_{i}^{0} v-\omega_{i}^{0} \mathbf{x}_{i}^{0} v^{2}
$$

with the zero point energy being set to zero, $\omega_{i}^{0}$ and $\omega_{i}^{0} x_{i}^{0}$ are related to $\omega_{i}$ and $\omega_{i} x_{i}$ in the following ways: $\quad \omega_{\mathrm{i}}=\omega_{\mathrm{i}}^{0}+\omega_{\mathrm{i}}^{0} \mathbf{x}_{\mathrm{i}}^{0}$ and $\omega_{\mathrm{i}}^{0} \mathbf{x}_{\mathrm{i}}^{0}=\omega_{\mathrm{i}} \mathbf{x}_{\mathrm{i}}$. Here $\omega_{\mathrm{i}}$ is the fundamental vibration frequency in $\mathrm{cm}^{-1}$ and $\omega_{i} x_{i}$ is the quadratic anharmonicity constant for the ith normal vibrational mode.

From the results, we conclude not only that all three totally symmetric vibrational modes were excited, but that agrees with the theoretical calculations by Domcke et al., 8 not with the results of the calculations by Takeshita ${ }^{13}$, but also that the non-totally symmetric mode $v_{4}$ was excited. The excitation of odd quanta of the $v_{4}$ mode is an indication that the molecular ion might have an equilibrium geometry other than planar. This can be argued 
qualitatively using the Walsh diagram approach and compared very favorably with the electronic absorption spectrum correspond to the transition of $\widetilde{A}{ }^{1} A_{2} \leftarrow \widetilde{X}{ }^{1} A_{1}$ in the UV absorption spectrum. In the photoelectron spectrum, this corresponds to the removal of an electron from the $b_{2}\left(n_{0}\right)$ orbital, thus reducing the stabilization energy gained by having two electrons in the planar form. This also reduced the repulsion between the hydrogen atoms and the oxygen atom. There are MO calculations ${ }^{13,44}$ indicating that in the $\widetilde{\mathrm{X}}^{2} \mathrm{~B}_{2}$ state of the $\mathrm{H}_{2} \mathrm{CO}^{+}$, the $\mathrm{HCO}$ angle decreases slightly $\left(\sim 5^{\circ}\right)$ from the neutral molecular $\widetilde{\mathrm{X}}$ ${ }^{1} \mathrm{~A}_{1}$ state. From their $a b$ initio calculation results 45 , Buenker and Peyerimhoff concluded that molecules with 10 and 11 valence electrons are less strongly planar compared to those with 12 upon removal of electrons from the $n$ species that are the nonbonding orbitals. But we expect the deviation from the planar equilibrium geometry to be very small.

The vibrational progressions in the observed spectra confirmed the earlier $a b$ initio calculations with a many body approach by W. Domcke et al., 8 but disagree with the $a b$ initio calculations by Takeshita 13 concerning the excitation of the $v_{1}$ mode. The anharmonicity would be too big for $v_{2}$ if we assign the feature that appeared at $11.2086 \mathrm{eV}$ to be the $v_{2}=2$ peak, according to Takeshita. It is noteworthy that different vibrational modes get excited to different extents between formaldehyde and deuterated formaldehyde. This gave some indications that the ionic ground state potential energy surfaces could be quite different for these isotopic compounds even if the neutral ground state potential energy surfaces might not be much different.

The vibrational autocorrelation function calculated using the formalism and procedure described in section $\mathrm{D}$ for the $\widetilde{\mathrm{X}} 1^{2} \mathrm{~B}_{2}$ states of $\mathrm{H}_{2} \mathrm{CO}^{+}$and $\mathrm{D}_{2} \mathrm{CO}^{+}$are presented in Figure 5 and Figure 6, respectively. The complex beat pattern of the $|C(t)|^{2}$ curve is the result of four different vibrational modes, after the initial drop, all having different phase and probably all having different phase space distances to travel as well. But the overall 
monotonic decay trend in these $\left|C_{a v}(t)\right|^{2}$ curves does indicate that the $\widetilde{\mathrm{X}} 1^{2} \mathrm{~B}_{2}$ state is in a stable configuration in the Franck-Condon region accessible by the photoionization event. It should be noticed that there were no deep minimums in the correlation functions, which are charateristics of a wave packet prepared through a predominately adiabatic transition. The initially prepared wave packet is mostly localized around the minimum of the upper potential energy surfaces, and weakly oscillates around this region, retaining substantial correlation at all times. The absence of the return of major oscillation strength can be attributed to the excitation of many modes which all having different phases after the initial drop and all having different phase spaces to travel.

\section{Second Band, $\tilde{A}^{2} \mathbf{B}_{1}$ State}

The second bands of $\mathrm{H}_{2} \mathrm{CO}^{+}$and $\mathrm{D}_{2} \mathrm{CO}^{+}$are shown in Figure 7 and Figure 8 , separately. The vibrational progressions observed are summarized in Table III. Here the small $\mathrm{N}_{2}^{+} \tilde{\mathrm{X}}$ peak presented in the $\mathrm{D}_{2} \mathrm{CO}^{+}$spectra, resulting from the main chamber background, makes the absolute IEs reportable to an accuracy of $\pm 1.0 \mathrm{meV}$ which is limited by the energy scale drift during the scans. It ought to be noted that the vibrational couplings are different for $\mathrm{H}_{2} \mathrm{CO}^{+}$and $\mathrm{D}_{2} \mathrm{CO}^{+}$. In $\mathrm{H}_{2} \mathrm{CO}^{+}$, the major vibrational excitation is the $\mathrm{V}_{2}$ mode together with several quanta of $\mathrm{V}_{3}$ excited as well. In $\mathrm{D}_{2} \mathrm{CO}^{+}$, on the other hand, the major vibrational excitation is the $v_{2}$ mode, but the coupling is different between vibrational modes: here it is the $v_{1}$ mode that gets excited along with the major excitation of the $V_{2}$ mode. These agree fairly well with the theoretical calculations by Domcke et al. 8 when many-body effects are included, and did not agree with the $a b$ initio calculations by Takeshita with a Roothaan's restricted Hartree-Fork approach. In Domcke et al.'s calculation for both isotopic species, the coupling of the $v_{2}$ modes are very strong. They are 2.792 and 2.611 for $\mathrm{H}_{2} \mathrm{CO}^{+}$and $\mathrm{D}_{2} \mathrm{CO}^{+}$, respectively. The coupling of $\mathrm{V}_{3}(0.270)$ is much stronger than that of $\mathrm{V}_{1}(0.036)$ in $\mathrm{H}_{2} \mathrm{CO}^{+}$, while in $\mathrm{D}_{2} \mathrm{CO}^{+}$the coupling of $v_{1}$ 
(0.414) is much stronger than that of $v_{3}(0.011)$. Also, the length of the vibrational progressions is slightly different, $\mathrm{H}_{2} \mathrm{CO}^{+}$has a slightly shorter progression than $\mathrm{D}_{2} \mathrm{CO}^{+}$(in the case of $\mathrm{D}_{2} \mathrm{CO}^{+}$, the $\tilde{\mathrm{A}}$ state vibrational progression would extend to $\mathrm{V}_{2}=10$ if it were not obscured by the $\mathrm{N}_{2}^{+} \tilde{\mathrm{X}}$ state peak). This is an indication that the ionic potential energy surfaces are slightly different for the isotopic compounds.

The difference in vibrational excitations has its origin in the different vibronic coupling coefficients (also called vibrational couplings by Domcke et al. ${ }^{8}$ ) for the isotopic compounds as discussed in section $\mathrm{C}$ of this chapter. As pointed by Domcke et al. ${ }^{8}$ in their $a b$ initio many-body approach calculations, the vibrational coupling can be very different for isotopic compounds, since the coupling coefficients are mass-dependent (the kinematic mutrix, that transforms from normal to internal coordinates, of $\mathrm{D}_{2} \mathrm{CO}$ differs considerably from that of $\mathrm{H}_{2} \mathrm{CO}$ ). It is expected that different vibrational modes will be excited to different extent for isotopic molecules. This also indicates that parts of the ionic potential energy surfaces of the $\tilde{A}^{2} B_{1}$ state accessible by the photoionization excitation for $\mathrm{H}_{2} \mathrm{CO}^{+}$and $\mathrm{D}_{2} \mathrm{CO}^{+}$are different, especially along different normal coordinates.

The strong excitation of the $v_{2}$ mode is an indication that a strongly bonding electron is being ejected. This agrees with previous experimental and theoretical results. But the appearances of the odd $v_{4}$ mode with very weak intensity, just as in the ionic ground state, is an indication that the molecular ions might have a nonplanar equilibrium geometry in the $\tilde{\mathrm{A}}^{2} \mathrm{~B}_{1}$ state. This could be especially important in $\mathrm{D}_{2} \mathrm{CO}$, where the $\mathrm{V}_{4}=$ 1,2 peaks are comparably stronger than in $\mathrm{H}_{2} \mathrm{CO}^{+}$. Just as in their ionic ground states, the deviation from the planar equilibrium geometry in the $\tilde{A}^{2} B_{1}$ state is expected to be very small.

The vibrational autocorrelation functions for the $\tilde{\mathrm{A}}^{2} \mathrm{~B}_{1}$ state of $\mathrm{H}_{2} \mathrm{CO}^{+}$and $\mathrm{D}_{2} \mathrm{CO}^{+}$ after corrections for the instrument response function and rotational broadening are shown 
in Figure 9 and Figure 10. The overall shapes of the correlation function for $\mathrm{H}_{2} \mathrm{CO}^{+}$and $\mathrm{D}_{2} \mathrm{CO}+$ is very similar, showing only one major progression with a period of about 28.5 fs, which is the vibrational period of the $v_{2}$ mode. But differences are to be noted as well. In the case of $\mathrm{H}_{2} \mathrm{CO}^{+}$, the correlation function retains its major oscillation up to about 170 fs. Beyond that, the wave packet spreads out and multiple peaks appear in the correlation function. In $\mathrm{D}_{2} \mathrm{CO}^{+}$, the correlation function retains its major oscillation up to almost 300 fs. The correlation strength in $\mathrm{C}(\mathrm{t})$ is also much smaller in $\mathrm{H}_{2} \mathrm{CO}^{+} \sim 0.58$, compared with $\mathrm{D}_{2} \mathrm{CO}^{+} \sim 0.74$. Also, the peaks in the $\mathrm{D}_{2} \mathrm{CO}^{+}$correlation function are much sharper than those in the $\mathrm{H}_{2} \mathrm{CO}^{+}$correlation function. The observed differences in the appearance of the correlation function gave us some indications about the differences in the ionic potential energy surfaces between $\mathrm{H}_{2} \mathrm{CO}^{+}$and $\mathrm{D}_{2} \mathrm{CO}^{+}$. First, the anharmonicity, which is known to cause the spreading of the correlation function peaks, must be smaller in $\mathrm{D}_{2} \mathrm{CO}^{+}$than in $\mathrm{H}_{2} \mathrm{CO}+$ along the $\mathrm{Q}_{2}$ normal coordinates. In fact the anharmonicity constants are $10.0 \mathrm{~cm}^{-1}$ and $5.7 \mathrm{~cm}^{-1}$ for $\mathrm{H}_{2} \mathrm{CO}^{+}$and $\mathrm{D}_{2} \mathrm{CO}^{+}$, respectively. Second, $\mathrm{D}_{2} \mathrm{CO}^{+}$in the $\tilde{\mathrm{A}}^{2} \mathrm{~B}_{1}$ state is more tightly bonded than $\mathrm{H}_{2} \mathrm{CO}^{+}$: the correlation function of $\mathrm{D}_{2} \mathrm{CO}^{+}$retains a higher degree of correlation in longer times, and this could also imply that the initial wave packet of $\mathrm{D}_{2} \mathrm{CO}^{+}$spans a relatively smaller portion of the phase space on the upper potential energy surface.

The modulations of the correlation function were caused by two sources. The first one is the small spin-orbital splittings in this band, since it is a $\pi$ bonding orbital electron that was ejected. The FWHM for peaks in this band was close to $12.5 \mathrm{meV}$, which implies that the spin-orbital splitting is $\leq 2 \mathrm{meV}$. The second is the excitation of additional vibrational modes other than $v_{2}$ in both $\mathrm{H}_{2} \mathrm{CO}^{+}$and $\mathrm{D}_{2} \mathrm{CO}^{+}$. This gives the doublet- and multiplet-like structures in the photoelectron spectra that produce a beat pattem that modulates the correlation function. After removing these two effects by the method of 
Lorquet, ${ }^{28 \mathrm{a}}$ that involved dividing the $C(t)$ obtained with $|\cos \Omega t / 2 \hbar|$, the values of $\Omega$ were chosen to give an overall monotonic decay $\mathrm{C}(\mathrm{t})$ by carefully removing the singular points in the cosine functions. The corrected vibrational autocorrelation functions of the $\tilde{\mathrm{A}}^{2} \mathrm{~B}_{1}$ state of $\mathrm{H}_{2} \mathrm{CO}^{+}$and $\mathrm{D}_{2} \mathrm{CO}^{+}$are shown at the bottom of Figure 9 and Figure 10 in forms of $|C(t)|^{2}$. The $\left|C_{a v}(t)\right|^{2}$ fittings of the $|C(t)|^{2}$ were also included there.

It can be seen from Figure 9 and Figure 10 that the overall decay behavior of $\mathrm{H}_{2} \mathrm{CO}^{+}$and $\mathrm{D}_{2} \mathrm{CO}^{+}$in the $\tilde{\mathrm{A}}^{2} \mathrm{~B}_{1}$ state is very similar, but the isotope effect does manifest itself in that $\mathrm{D}_{2} \mathrm{CO}^{+}$has a relatively much slower decay rate after the initial dephasing than $\mathrm{H}_{2} \mathrm{CO}^{+}$, and the initial dephasing rates are different as well. As pointed by Rustić28b, this initial (before the first half vibrational period) dephasing rate depends very sensitively on the differences between the ionic state and the neutral ground state equilibrium geometries. This is yet another possible indication that in the $\tilde{\mathrm{A}}^{2} \mathrm{~B}_{1}$ state of $\mathrm{H}_{2} \mathrm{CO}^{+}$and $\mathrm{D}_{2} \mathrm{CO}^{+}$, the equilibrium geometries might be slightly different from the planar ones. The wave packet is displaced substantially from the minimum of the upper potential energy surface which is consistant with the observed long vibrational progressions, and indicating a strong bonding electron being ejected. The wave packet oscillated with large amplitude on the upper potential energy surface which accounts for the deep minimums in the correlation functions. The time window, over which we could deduce useful information on the intramolecular dynamics was limited by the finite resolution achievable. As shown in this state after removal of all the possible broadening effects, the time window was about 200 to $300 \mathrm{fs}$ wide. This corresponds to an effective resolution of $\leq 3 \mathrm{meV}$, which is to be compared with the estimated helium I $\alpha$ resonance line width produced by the lamp, to be $\leq$ $2 \mathrm{meV}$.

3. Third Band, $\widetilde{\mathrm{B}}{ }^{2} \mathrm{~A}_{1}$ State

The third bands of $\mathrm{H}_{2} \mathrm{CO}^{+}$and $\mathrm{D}_{2} \mathrm{CO}^{+}$are shown in Figure 11 and Figure 12. The 
vibrational progressions observed are summarized in Table IV. The presence of the small Ar+ peaks in the $\mathrm{H}_{2} \mathrm{CO}+$ band, resulting from the main chamber background from frequent calibration runs, makes the absolute IEs for this band reportable to an accuracy of $\pm 1.0 \mathrm{meV}$, as discussed in the discussion of the $\tilde{\mathrm{A}}^{2} \mathrm{~B}_{1}$ band of $\mathrm{D}_{2} \mathrm{CO}^{+}$. As we have pointed out in the discussion of the $\tilde{\mathrm{A}}^{2} \mathrm{~B}_{1}$ band, the vibrational couplings are different for different isotopic compounds. In previous experimental and theoretical studies, it was concluded that the simplicity of this band in $\mathrm{H}_{2} \mathrm{CO}^{+}$was due to the accident degeneracy of the $V_{2}$ and $v_{3}$ normal vibrational modes. The removal of this degeneracy in $\mathrm{D}_{2} \mathrm{CO}^{+}$was used as evidence for this interpretation.

The $V_{2}$ vibrational frequency is $1304.5 \mathrm{~cm}^{-1}$ for $\mathrm{H}_{2} \mathrm{CO}^{+}$and $1311.1 \mathrm{~cm}^{-1}$ for $\mathrm{D}_{2} \mathrm{CO}^{+}$as obtained for this state from the present study. The $v_{3}$ vibrational frequency, as determined from this band of $\mathrm{D}_{2} \mathrm{CO}^{+}$, is $957.4 \mathrm{~cm}^{-1}$, for $\mathrm{H}_{2} \mathrm{CO}^{+}$is $1304.5 \mathrm{~cm}^{-1}$ (assuming here $v_{3}=V_{2}$ ). This reduction upon deuteration seems reasonable when compared with the neutral $\tilde{\mathrm{X}}^{1} \mathrm{~A}_{1}$ ground state of $\mathrm{H}_{2} \mathrm{CO}^{+}$and $\mathrm{D}_{2} \mathrm{CO}^{+}$with $\mathrm{V}_{3}=1500.2 \mathrm{~cm}^{-1}$, and $1105.7 \mathrm{~cm}^{-1}$ respectively. The FWHM in the spectra shown for $\mathrm{H}_{2} \mathrm{CO}^{+}$and $\mathrm{D}_{2} \mathrm{CO}^{+}$is about $12 \mathrm{meV}$, which is very close to the measured instrumental resolution. Unless $v_{2}$ and $v_{3}$ were truly degenerate to within $1 \mathrm{meV}$ or less, we would have seen the broadening of the peaks in $\mathrm{H}_{2} \mathrm{CO}^{+}$. Upon close examination, it is the other way around, however, the peaks in $\mathrm{D}_{2} \mathrm{CO}^{+}$are slightly broader than those in $\mathrm{H}_{2} \mathrm{CO}^{+}$. Theoretical calculations ${ }^{8}$ indicate that in the $\widetilde{B}{ }^{2} \mathrm{~A}_{1}$ state of $\mathrm{H}_{2} \mathrm{CO}^{+}$, the coupling of $\mathrm{V}_{3}(0.301)$ is much weaker than that of $\mathrm{V}_{2}$ (1.156). In $\mathrm{D}_{2} \mathrm{CO}^{+}$, the coupling of $\mathrm{V}_{3}$ is slightly larger $(0.855)$ than that of $\mathrm{V}_{2}(0.765)$ when many body effects are included, according to these authors. It is possible that the coupling of $\mathrm{V}_{3}$ in the $\widetilde{\mathrm{B}}^{2} \mathrm{~A}_{1}$ state of $\mathrm{H}_{2} \mathrm{CO}+$ is so weak that we are actually seeing only the progression of the $V_{2}$ vibration. While in the $\widetilde{B}^{2} A_{1}$ state of $D_{2} C O^{+}$both $V_{3}$ and $V_{2}$ are coupled strongly, both vibrational progressions are seen. We thus tentatively assigned the vibrational progressions in the $\widetilde{\mathrm{B}}^{2} \mathrm{~A}_{1}$ state of $\mathrm{H}_{2} \mathrm{CO}^{+}$to the $\nu_{2}$ mode only. The present 
assignment and the observed AIEs, VIEs and vibrational progressions definitely support the assignment of Brundle et al. 12 and all theoretical calculations ${ }^{3-13}$ available that this band is the $\widetilde{\mathrm{B}}{ }^{2} \mathrm{~A}_{1}$ state of formaldehyde.

The vibrational correlation functions for the $\widetilde{\mathrm{B}}{ }^{2} \mathrm{~A}_{1}$ state of $\mathrm{H}_{2} \mathrm{CO}^{+}$and $\mathrm{D}_{2} \mathrm{CO}^{+}$, calculated by digitally remove the $\widetilde{\mathrm{C}}{ }^{2}{ }^{2} \mathrm{~B}_{2}$ bands' contributions, are shown in Figure 13 and Figure 14. It can be seen from Figure 13 that for $\mathrm{H}_{2} \mathrm{CO}^{+}$, there is only one major progression, with a period of $\sim 40$ fs. The anharmonicity starts to spread the correlation function around $t=100 \mathrm{fs}$. The slow decay of the correlation function indicates that this state is in a very stable configuration regarding deformations along the $Q_{2}$ normal coordinate. In the case of $\mathrm{D}_{2} \mathrm{CO}^{+}$, it can be seen that this state is in a very stable configuration regarding deformations along both the $\mathrm{Q}_{2}$ and the $\mathrm{Q}_{3}$ normal coordinates. The overall rate of decay as shown by the $\left|C_{a v}(t)\right|^{2}$ of both isotopic compounds, is very similar qualitatively. Quantitatively speaking, in this electronic state $\mathrm{D}_{2} \mathrm{CO}+$ is more stable than $\mathrm{H}_{2} \mathrm{CO}^{+}$. In $\mathrm{D}_{2} \mathrm{CO}^{+}$, the excitation of both the $v_{2}$ and $v_{3}$ mode gives rise to the doublet- and multiplet-like structures in the photoelectron spectrum. These doublet and multiplet structures in the energy domain, when Fourier transformed give rise to the beat pattern observed in the correlation function, as discussed in previous section, and was not corrected for this band. It is to be noted here that the initial rates of decay for $\mathrm{H}_{2} \mathrm{CO}^{+}$and $\mathrm{D}_{2} \mathrm{CO}^{+}$in this state are very different, indicating, again, possibly very different potential energy surfaces for $\mathrm{H}_{2} \mathrm{CO}^{+}$and $\mathrm{D}_{2} \mathrm{CO}^{+}$in the $\widetilde{\mathrm{B}}{ }^{2} \mathrm{~A}_{1}$ state. The deep valleys in the correlation functions, as discussed in previous section of the $\tilde{\mathrm{A}}{ }^{2} \mathrm{~B}_{1}$ state correlation function, indicate a substantially displaced wave packet from the upper potential energy minimums.

\section{Fourth Band, $\widetilde{\mathrm{C}} 2^{2} \mathrm{~B}_{2}$ State}

The fourth band of $\mathrm{H}_{2} \mathrm{CO}^{+}$was shown in Figure 11 , together with the $\widetilde{\mathrm{B}}{ }^{2} \mathrm{~A}_{1}$ state. 
The $\widetilde{C}{ }^{2} \mathrm{~B}_{2}$ state of $\mathrm{D}_{2} \mathrm{CO}^{+}$is shown separately in Figure 15 . Even with sufficient supersonic cooling in the present high resolution study, the complexity of this band for both $\mathrm{H}_{2} \mathrm{CO}^{+}$and $\mathrm{D}_{2} \mathrm{CO}^{+}$cannot be fully untangled. The vibrational assignment could only be regarded as tentative, especially in the case of $\mathrm{D}_{2} \mathrm{CO}^{+}$. As pointed out by Turner et al. in their original studies, this complexity might have been caused by the crossing of another repulsive state with the $\widetilde{\mathrm{C}} 2^{2} \mathrm{~B}_{2}$ state. Theoretical calculations do indicate that the density of the electronic states increase rapidly with the energy. In the energy range 16.4-17 eV (where the fourth band appears in the photoelectron spectrum) above the ground state of the neutral molecule, four electronic states are present and their potential energy surfaces intersect. ${ }^{18} \mathrm{In}_{2} \mathrm{CO}^{+}$, the major progressions are assigned to the excitations of $v_{1}$ and $\mathrm{V}_{3}$ modes, based largely on the many-body calculations by Domcke et al. ${ }^{8}$ The adiabatic ionization potential was tentatively assigned as $\mathbf{A I E}=16.2395 \mathrm{eV}$. In $\mathrm{D}_{2} \mathrm{CO}^{+}$, the major progressions are much weaker compared with the corresponding ones in $\mathrm{H}_{2} \mathrm{CO}^{+}$, and were assigned to $v_{1}, v_{2}$, and $v_{3}$ modes. The adiabatic ionization potential, however, can only be estimated to be $\leq 16.435 \pm 0.02 \mathrm{eV}$.

The vibrational correlation functions for the $\widetilde{\mathrm{C}} 2^{2} \mathrm{~B}_{2}$ state of $\mathrm{H}_{2} \mathrm{CO}^{+}$and $\mathrm{D}_{2} \mathrm{CO}+$ are presented in Figure 16 and Figure 17, respectively. The major feature of the correlation functions for this band is the extremely fast loss of correlation strength as manifested by the correlation functions obtained. Beyond the initial drop of the correlation function at $\sim 5 \mathrm{fs}$, there are no recognizable major oscillations that are above noise level. This and the appearance of the broad photoelectron bands indicate that the molecular ions created by the photoionization event in this state undergo very fast intramolecular dynamic processes. The wave packet never regains its initial shape and returns to its initial position on the potential energy surfaces. Even the initial drup in the correlation function is much faster when compared with other bands of these molecular ions. This, in the spirit of Ruscici, 
indicates that the ionic state equilibrium geometry is substantially different from not only the ground state equilibrium geometry of the neutral molecule, but also other ionic electronic states as well. Isotope effect showed up strongly here, the correlation strength of $\mathrm{H}_{2} \mathrm{CO}^{+}$drops much faster than that of $\mathrm{D}_{2} \mathrm{CO}^{+}$

\section{F. Conclusion}

Rotationally cold photoelectron spectra of $\mathrm{H}_{2} \mathrm{CO}$ and $\mathrm{D}_{2} \mathrm{CO}$ with much improved resolution have allowed each of the four ionic electronic states accessible by the helium I $\alpha$ radiation to be characterized in great detail. The assignment of the third and fourth ionic states to the $\widetilde{\mathrm{B}}{ }^{2} \mathrm{~A}_{1}$ and $\widetilde{\mathrm{C}}{ }^{2} \mathrm{~B}_{2}$ states confirms the results of Brundle et al., and all theoretical calculations available. For the $\widetilde{X} 1^{2} \mathrm{~B}_{2}$ and the $\tilde{\mathrm{A}}^{2} \mathrm{~B}_{1}$ state, the excitation of odd quanta of the non totally symmetric vibrational mode $\left(V_{4}\right)$ indicates the possibilities of non planar equilibrium geometries for these two states. Strong isotope effects were observed in the vibronic couplings in the $\widetilde{\mathrm{A}}{ }^{2} \mathrm{~B}_{1}$ and $\widetilde{\mathrm{B}}{ }^{2} \mathrm{~A}_{1}$ states. This indicates that for isotopic compounds the details of the ionic potential energy surfaces for the same electronic state can be very different. Rotationally resolved spectroscopic studies on $\mathrm{H}_{2} \mathrm{CO}^{+}$and $\mathrm{D}_{2} \mathrm{CO}^{+}$in its various ionic states will be very desirable to confirm the equilibrium geometries of these molecules in these ionic states.

Vibrational correlation functions were derived for all four electronic states. The variations between the isotopic species' correlation functions were attributed primarily to the differences in the details of the potential energy surfaces and in the phase space spanned by the initially formed wave packets. This isotope effect on vibronic coupling is especially evident in the case of the first and second excited states of formaldehyde, where completely different vibrational progressions were observed in the same electronic state of different isotopic compounds. Ultra fast intramolecular decay mechanisms were evident for the $\widetilde{\mathrm{C}}$ ${ }^{2} \mathrm{~B}_{2}$ state of each isotopic ions. The decaying of this state occurred in a time scale of less 
than one period of $\mathrm{C}-\mathrm{H}$ stretching vibrational, supporting the relaxation pathway by predissociation through crossing by close lying and intersecting electronic states in that energy range. Further theoretical treatment on this state will be particularly important to understand the details of the ultra fast intramolecular dynamics evident in this investigation. 


\section{References:}

1. A. D. Baker, C. Baker, C. R. Brundle, and D. W. Turner, Int. J. Mass Spectrom. Ion Phys., 1 , 285(1968)

2. D. W. Turner, A. D. Baker, and C. R. Brundle, Molecular Photoelectron Spectroscopy, John Wiley \& Sons, London, p135-137(1970)

3. W. V. Niessen, G. Bieri, and L. Åsbrink, J. Elect. Spect. Relat. Phenom. 21, 175(1980)

4. K. Kimura, S. Katsumata, Y. Achiba, T. Yamazak, and S. Iwata, Handbook of Hel Photoelectron Spectra of Fundamental Organic Molecules, Halsted, New York, (1981)

5. D. P. Chong, F. G. Herring, and D. Mcwilliams, J. Chem. Phys., 61, 958(1974)

6. L. S. Cederbaum, G. Hohlneicher, and W. V. Niessen, Mol. Phys., 26, 1405(1973)

7. L. S. Cederbaum, and W. Domcke, J. Chem. Phys., 64(2), 603(1976)

8. D. Domcke, and L. S. Cederbaum, ibid, 64(2), 612(1976)

9. J. F. Blanke, and J. Overend, in Vibrational Spectra and Structure, Yol. 7 , p270-315(1978), ed. by J. R. Durig, Elsevier Sci. Pub. Com., New York

10a. D. C. Moule, Yibrational Spectra and Structure, Vol.6, p228-271(1977), ed. by J. R. Durig, Elsevier Sci. Pub. Com., New York.

10b. I. W. Levin, and R. A. R. Pearce, in Vibrational Spectra and Structure, Vol,4, ed. by J. R. Durig, Elsevier Sci. Pub. Com., New York, p102(1975).

11. C. R. Brundle, and D. W. Turner, Chem. Comm., 341(1967)

12. C. R. Brundle, M. B. Robin, N. A. Kuebler, and H. Basch, J. Am. Chem. Soc., 24, 1451(1972)

13. K. Takeshita, J. Chem. Phys., 24(11), 7259(1991)

14. P. M. Guyon, W. A. Chupka, J. Berkowitz, ibid, 64, 1419(1976) 
15. R. Bombach, J. Dannacher, J-P Stadelmann, and J. Vogt, Chem. Phys. Lett., 76(3), 429(1980)

16. S. V. Andreyev, V. S. Antonov, I. N. Knyazev, and V. S. Letokhov, Chem. Phys. Lett., 45(1), 166(1977).

17. R. Bombach, J. Dannacher, J-P Stadelmann, and J. Vogt, Int. J. Mass Spectrom. Ion Phys., 40, 275(1981).

18. M. Van Pires, C. Galloy, and J. C. Lorquet, J. Chem. Phys., 69, 3242(1978).

19. S. T. Hood, A. Hamnett, and C. E. Brion, Chem. Phys. Lett., 41(3), 428(1976).

20. R. S. Mulliken, Rev. Mod. Phys., 14, 204(1942).

21a. A. D. Walsh, J. Chem. Soc, 2260(1953).

21b. D. C. Moule, A. D. Walsh, Chem. Rev., 75(1), 67-84(1975).

22. R.J. Buenker, S.D. Peyerimhoff, Chem. Rev., 74(2), 127-188 (1974).

23 E. J. Heller, J. Chem. Phys., 68, 2066(1978).

24. E. J. Heller, E. B. Stechel, M. J. Davis, ibid, 73(10), 4720(1980).

25. E. J. Heller, Acc. Chem. Res., 14, 368(1981).

26. E. J. Heller, R. Sundberg, and D. Tanner, J. Chem.. Phys., 76, 1822(1982).

27. B. Layh-Nihaut, and J. C. Lorquet, ibid, 88(9), 5606(1988).

28a. A. J. Lorquet, J. C. Lorquet, J. Delwiche, and M. J. Hubin-Franskin, ibid, 76(10), 4692(1982).

28b. B. Ruscić, ibid, 85(7), 3776(1986).

29. F. Remacle, M. Desoute-Leconmte, and J. C. Lorquet, ibid, 21(7), 4155(1989).

30. J. E. Pollard, D. J. Trevor, J. E. Reutt, Y. T. Lee, and D. A. Shirley, ibid, 81(2) 5302(1984).

31. J. E. Reutt, L. S. Wang, J. E. Pollard, D. J. Trevor, Y. T. Lee, and D. A. Shirley, ibid, 84(6), 3022(1986).

32. J. E. Reutt, L. S. Wang, Y. T. Lee, D. A. Shirley, ibid, 85(12), 6928(1986). 
33. P. R. Keller, J. W. Taylor, F. A. Grimm, and T. A. Carlson, Chem. Phys., 20 , 147-153(1984).

34a. J. E. Pollard, D. J. Trevor, Y. T. Lee, and D. A. Shirley, Rev. Sci. Instrum., 52, p1837 (1981).

34b. J. E. Pollard, Ph.D. Thesis, Department of Chemistry, The University of Califomia at Berkeley (1982).

35. J. E. Reutt, Ph.D. Thesis, Department of Chemistry, The University of California at Berkeley (1986).

36a. R. Spencer, and W. Wild, L. Chem.Soc, London, 338-340(1935).

36b. R. Spence, and W. Wild, L.Chem. Soc, London, 506-509(1935).

37. W. E Polik, Ph.D. Thesis, Department of Chemistry, The University of California at Berkeley (1988)

38. A. Lofthus, and P.H. Krupenie, J. Phys. Chem. Ref. Data, 6, 113 (1977)

39. Atomic Energy Levels, Vol. I, II, III, ed. by C. E. Moore, (National Bureau of Standards, Washington D.C., 1958).

40a. M. Born, J.R. Oppenheimer, Ann. Phys.(Lepzig), 84, 457-484 (1927).

40b. M. Born, Nachr. Akad. Wiss. Gottingen, Math.-Phys. KL, IIa, P1 (1951).

40c. M. Born, K. Huang, Dynamics of Crystal Lattice, Oxford U. Press, NY., (1954).

41. J. W. Rabalais, Principles of Ultraviolet Photoelectron Spectroscopy, John Wiley \& Sons, New York (1979)

42. H. Köppel, W. Domcke, and L. S. Cederbaum, Adv. Chem. Phys, Vol. LVII, p59-246(1984)

43. E. Oran Brighan, The Fast Fourier Transform, (Prentice-Hall, Englewood Cliffs), p164(1974).

44. R. J. Buenker, and S. D. Peyerimhoff, Chem. Rev., 7(2), 127-188(1974)

45. R. J. Buenker, and S. D. Peyerimhoff, J. Chem. Phys., 53, 1368(1970) 
46a. J. I Steinfeld, An_Introduction to Modern Molecular Speciroscopy 2nd ed., The MIT Press, Cambridge, MA(1985) p133.

46b. G. Herzberg, Molecular Snectra \& Molecular Structure, L. Spectra of Diatomic Molecules, Van Norstrand Reinhold Com., New York, p93(1950).

47. D. E. Reisner, R. W. Field, J. L. Kinsey, and H-L Dai, J. Chem. Phys., 80(12), 5968-5978(1984).

48. D. J. Clouthier, and D. A. Ramsay in Ann. Rev. Phys. Chem., 34, p31-58(1983). 


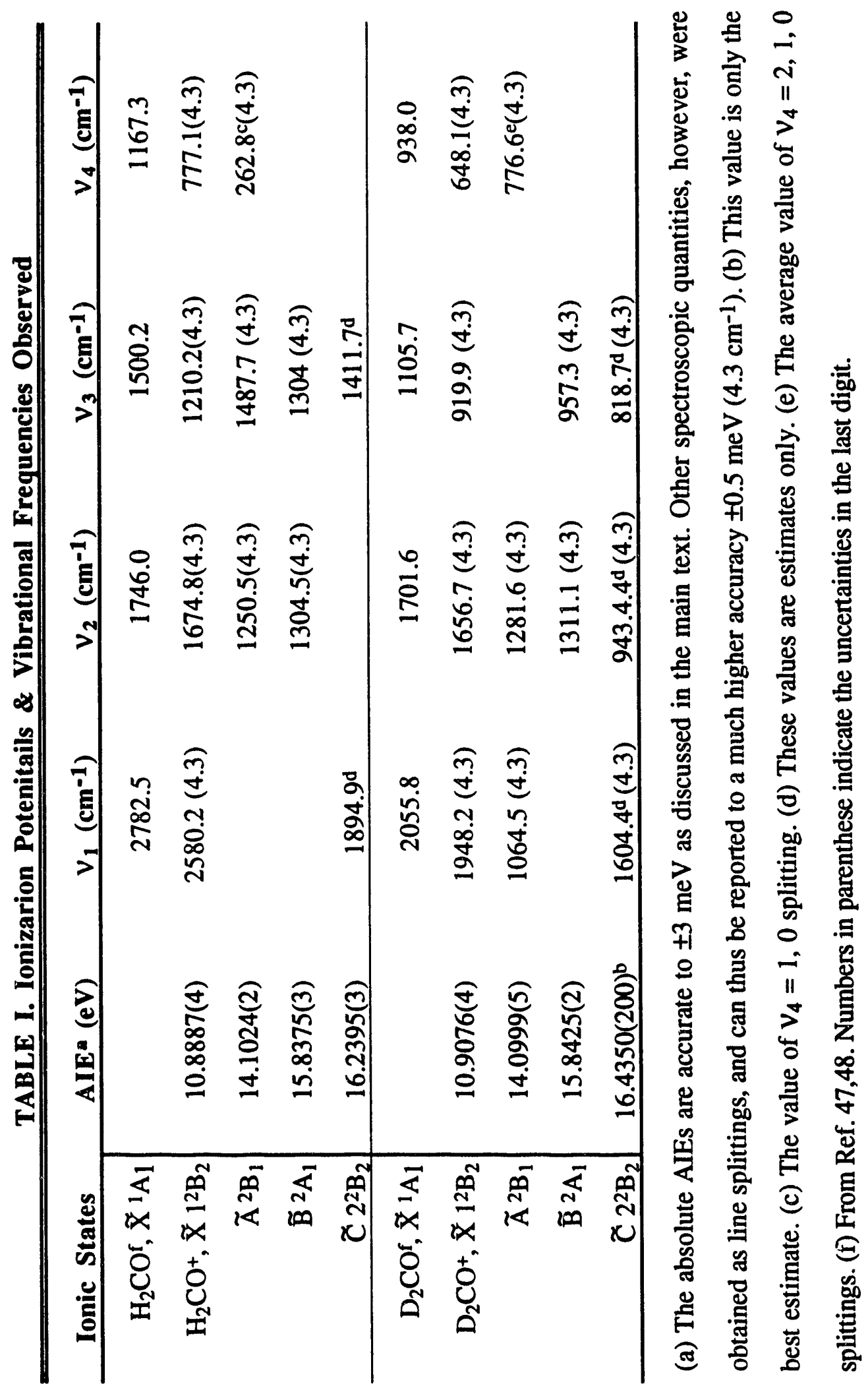


TABLE II. Vibrational Levels of the $\widetilde{\mathrm{X}} \mathbf{1}^{2} \mathrm{~B}_{2}$ State

\begin{tabular}{|c|c|c|c|}
\hline \multicolumn{2}{|c|}{$\mathrm{H}_{2} \mathrm{CO}^{+}$} & \multicolumn{2}{|c|}{$\mathrm{D}_{2} \mathrm{CO}^{+}$} \\
\hline $\operatorname{IE}^{\mathbf{a}}(\mathrm{eV})$ & $v_{1} v_{2} v_{3} v_{4}$ & IEa (eV) & $v_{1} v_{2} v_{3} v_{4}$ \\
\hline $10.8887(3)$ & 0000 & $10.9076(3)$ & 0000 \\
\hline $10.9834(5)$ & 0001 & $11.1456(7)$ & 1000 \\
\hline $11.0748(6)$ & 0002 & $11.3765(3)$ & 2000 \\
\hline $11.0380(8)$ & 0010 & $11.1116(8)$ & 0100 \\
\hline $11.1858(4)$ & 0020 & $11.3128(2)$ & 0200 \\
\hline $11.0940(2)$ & 0100 & $11.0178(5)$ & 0010 \\
\hline $11.2946(5)$ & 0200 & $11.1203(2)$ & 0020 \\
\hline $11.2086(3)$ & 1000 & $10.9887(4)$ & 0001 \\
\hline \multirow[t]{2}{*}{$11.2483(8)$} & 0110 & $11.0729(3)$ & 0002 \\
\hline & & $11.1584(6)$ & 0003 \\
\hline \multicolumn{2}{|c|}{$\mathrm{H}_{2} \mathrm{CO}^{+}$} & \multicolumn{2}{|c|}{$\mathrm{D}_{2} \mathrm{CO}^{+}$} \\
\hline$v_{n}(n=1-4)\left(\mathrm{cm}^{-1}\right)$ & $\omega_{\mathrm{e}} \mathrm{x}_{\mathrm{e}}\left(\mathrm{cm}^{-1}\right)$ & $V_{n}(n=1-4)\left(\mathrm{cm}^{-1}\right)$ & $\omega_{\mathrm{e}} \mathrm{x}_{\mathrm{e}}\left(\mathrm{cm}^{-1}\right)$ \\
\hline $2580.2(4.3)$ & & $1948.2(4.3)$ & $28.6(4.3)$ \\
\hline $1674.8(4.3)$ & $19.0(4.3)$ & $1656.7(4.3)$ & $11.3(4.3)$ \\
\hline $1210.2(4.3)$ & $6.1(4.3)$ & $919.9(4.3)$ & $31.1(4.3)$ \\
\hline $777.1(4.3)$ & $13.3(4.3)$ & $648.1(4.3)$ & $8.9(4.3)$ \\
\hline
\end{tabular}

(a) The absolute IEs are accurate to $\pm 3.0 \mathrm{meV}$ as discussed in the main text. Other spectroscopic constants, however, were obtained as line splittings, can thus be reported to a much higher accuracy $\pm 0.5 \mathrm{meV}\left(4.3 \mathrm{~cm}^{-1}\right)$. 
TABLE III. Vibrational Levels of the $\widetilde{A}^{2} B_{1}$ State

\begin{tabular}{|c|c|c|c|}
\hline $\mathrm{IE}^{\mathrm{a}} \mathrm{H}_{2} \mathrm{CO}^{+}(\mathrm{eV})$ & Vibrational & Progressions & $\left(V_{1} v_{2} V_{3} V_{4}\right)$ \\
\hline $14.1024(2)$ & & 0000 & \\
\hline $14.1280(3)$ & & & 0001 \\
\hline $14.2839(5)$ & & 0010 & \\
\hline $14.4724(5)$ & & 0020 & \\
\hline $14.6536(9)$ & & 0030 & \\
\hline $14.2560(2)$ & 0100 & & \\
\hline $14.4072(8)$ & 0200 & & \\
\hline $14.5562(3)$ & 0300 & & \\
\hline $14.7028(4)$ & 0400 & & \\
\hline $14.8471(3)$ & 0500 & & \\
\hline $14.9867(6)$ & 0600 & & \\
\hline $15.1264(2)$ & 0700 & & \\
\hline $15.2660(2)$ & 0800 & & \\
\hline $15.3963(4)$ & 0900 & & \\
\hline $14.2839(5)$ & & 0010 & \\
\hline $14.4375(4)$ & & 0110 & \\
\hline $14.5888(1)$ & & 0210 & \\
\hline $14.7354(2)$ & & 0310 & \\
\hline $14.8820(3)$ & & 0410 & \\
\hline $15.0239(9)$ & & 0510 & \\
\hline $15.1659(5)$ & & 0610 & \\
\hline $15.3032(5)$ & & 0710 & \\
\hline
\end{tabular}


Table III. continued

\begin{tabular}{l|ll}
\hline $15.4382(3)$ & & 0810 \\
$14.4724(5)$ & 0020 & \\
$14.6213(9)$ & 0120 & \\
$14.7726(6)$ & 0220 & \\
$14.9192(7)$ & 0320 & \\
$15.0658(8)$ & 0420 & \\
$15.2031(8)$ & 0520 & 0030 \\
$15.3474(7)$ & 0620 & 0130 \\
$14.6536(9)$ & & 0230 \\
$14.8073(9)$ & & 0330 \\
$14.9519(2)$ & $\omega_{\mathrm{e}} \mathrm{x}_{\mathrm{e}}=10.0(4.3) \mathrm{cm}^{-1}$ \\
$15.0918(6)$ & $\omega_{\mathrm{e}} \mathrm{x}_{\mathrm{e}}=0.6(4.3) \mathrm{cm}^{-1}$ \\
\hline
\end{tabular}

a. See table II. footnotes, also see main text

\begin{tabular}{|c|c|c|c|}
\hline $\mathrm{IE}^{\mathrm{a}} \mathrm{D}_{2} \mathrm{CO}^{+}(\mathrm{eV})$ & Vibrational & Progressions & $\left(V_{1} V_{2} V_{3} V_{4}\right)$ \\
\hline $14.0999(5)$ & & 0000 & \\
\hline $14.1366(4)$ & & & 0001 \\
\hline $14.2925(2)$ & & & 0002 \\
\hline $14.2283(6)$ & 1000 & & \\
\hline $14.3659(4)$ & 2000 & & \\
\hline $14.4874(8)$ & 3000 & & \\
\hline $14.6204(8)$ & 4000 & & \\
\hline $14.2604(6)$ & & 0100 & \\
\hline
\end{tabular}


Table III. continued

\begin{tabular}{|c|c|c|}
\hline $14.4163(9)$ & & 0200 \\
\hline $14.5723(2)$ & & 0030 \\
\hline $14.7282(5)$ & & 0040 \\
\hline $14.8750(2)$ & & 0050 \\
\hline $15.0309(4)$ & & 0060 \\
\hline $15.1763(1)$ & & 0070 \\
\hline $15.3282(1)$ & & 0080 \\
\hline $15.4755(9)$ & & 0090 \\
\hline $14.2283(6)$ & 1000 & \\
\hline $14.3865(8)$ & 1100 & \\
\hline $14.5425(1)$ & 1200 & \\
\hline $14.6984(4)$ & 1300 & \\
\hline $14.8497(8)$ & 1400 & \\
\hline $15.0034(2)$ & 1500 & \\
\hline $15.1473(4)$ & 1600 & \\
\hline $15.2982(4)$ & 1700 & \\
\hline $15.4456(2)$ & 1800 & \\
\hline $14.2283(6)$ & & 2000 \\
\hline $14.3659(84$ & & 2100 \\
\hline $14.5149(9)$ & & 2200 \\
\hline $14.6686(3)$ & & 2300 \\
\hline $14.8199(7)$ & & 2400 \\
\hline $14.9736(1)$ & & 2500 \\
\hline $15.1207(9)$ & & 2600 \\
\hline
\end{tabular}


Table III. continued

\begin{tabular}{|c|c|c|}
\hline $15.2694(2)$ & & 2700 \\
\hline $15.4156(4)$ & & 2800 \\
\hline $14.4874(8)$ & 3000 & \\
\hline $14.6411(2)$ & 3100 & \\
\hline $14.7924(6)$ & 3200 & \\
\hline $14.9438(2)$ & 3300 & \\
\hline $15.0959(2)$ & 3400 & \\
\hline $15.2407(8)$ & 3500 & \\
\hline $15.3881(6)$ & 3600 & \\
\hline $15.5330(4)$ & 3700 & \\
\hline $14.6204(8)$ & & 4000 \\
\hline $14.7718(2)$ & & 4100 \\
\hline $14.9185(1)$ & & 4200 \\
\hline $15.0749(3)$ & & 4300 \\
\hline $15.2233(2)$ & & 4400 \\
\hline $15.3656(8)$ & & 4500 \\
\hline $15.5055(6)$ & & 4600 \\
\hline \multicolumn{2}{|c|}{$v_{1}=1064.5(4.3) \mathrm{cm}^{-1}$} & $\omega_{\mathrm{e}} \mathrm{x}_{\mathrm{e}}=10.0(4.3) \mathrm{cm}^{-1}$ \\
\hline \multicolumn{2}{|c|}{$V_{2}=1281.6(4.3) \mathrm{cm}^{-1}$} & $\omega_{\mathrm{e}} \mathrm{x}_{\mathrm{e}}=5.7(4.3) \mathrm{cm}^{-1}$ \\
\hline
\end{tabular}

a. The accuracy of the IEs are $\pm 1.0 \mathrm{meV}$ for this state only: see main text for discussions. 
TABLE IV. Vibrational Levels of the $\widetilde{B}^{2} \mathrm{~A}_{1}$ State

\begin{tabular}{l|cc}
\hline \hline $\mathrm{IE}^{\mathrm{a}}$ of $\mathrm{H}_{2} \mathrm{CO}^{+}(\mathrm{eV})$ & \multicolumn{2}{c}{ Vibrational Progressions $\left(\mathrm{V}_{1} \mathrm{~V}_{2} \mathrm{~V}_{3}\right)$} \\
\hline $15.8375(2)$ & 010 & 000 \\
$15.9963(1)$ & 020 \\
$16.1541(1)$ & 030 \\
$16.3079(5)$ & 040 \\
$16.4593(2)$ & 050 \\
$16.6071(1)$ & 060 \\
$16.7506(6)$ & & $\omega_{\mathrm{e}} \mathrm{x}_{\mathrm{e}}=12.6(4.3) \mathrm{cm}^{-1}$ \\
\hline
\end{tabular}

(a) The accuracy of the IEs reported here are $\pm 1.0 \mathrm{meV}$ : see main text for discussions.

$\mathrm{IE}^{\mathrm{a}}$ of $\mathrm{D}_{2} \mathrm{CO}^{+}(\mathrm{eV})$

$15.8425(2)$

$15.9631(8)$

$16.0823(7)$

$16.1986(1)$

16.3111(9)

$16.4229(3)$

$16.5422(4)$

$16.6558(8)$

$16.0029(1)$

$16.1603(5)$

$16.3111(9)$

$16.4649(5)$

16.6062(1)
Vibrational Progressions $\left(V_{1} V_{2} V_{3}\right)$

000

001

002

003

004

005

006

007 
TABLE IV. continued

85

\begin{tabular}{|c|c|c|}
\hline $16.7497(6)$ & & 060 \\
\hline $15.9631(8)$ & 001 & \\
\hline $16.1235(7)$ & 011 & \\
\hline $16.2740(5)$ & 021 & \\
\hline $16.4240(5)$ & 031 & \\
\hline $16.5826(7)$ & 041 & \\
\hline $16.7324(6)$ & 051 & \\
\hline 16.ci2 237 & & 002 \\
\hline $16.2412(8)$ & & 012 \\
\hline $16.3972(6)$ & & 022 \\
\hline $16.5502(9)$ & & 032 \\
\hline $16.6954(2)$ & & 042 \\
\hline 16.19861 & 003 & \\
\hline $16.3575(3)$ & 013 & \\
\hline $16.5127(7)$ & 023 & \\
\hline \multicolumn{2}{|c|}{$v_{2}=1311.1(4.3) \mathrm{cm}^{-1}$} & 3) $\mathrm{cm}^{-1}$ \\
\hline \multicolumn{2}{|c|}{$V_{3}=957.3(4.3) \mathrm{cm}^{-1}$} & $\mathrm{~cm}^{-1}$ \\
\hline
\end{tabular}

a. See table II. footnotes. 
TABLE V. Vibrational Levels of the $\widetilde{C} 2^{2} B_{2}$ State

\begin{tabular}{|c|c|c|c|}
\hline $\mathrm{IE}^{\mathrm{a}}$ of $\mathrm{H}_{2} \mathrm{CO}^{+}(\mathrm{eV})$ & Vibrational & Progressions & $\left(V_{1} V_{2} V_{3}\right)$ \\
\hline $16.2395(3)$ & & 000 & \\
\hline $16.4811(5)$ & 100 & & \\
\hline $16.7065(3)$ & 200 & & \\
\hline $16.9304(2)$ & 300 & & \\
\hline $17.1581(2)$ & 400 & & \\
\hline $17.3832(1)$ & 500 & & \\
\hline $17.6041(3)$ & 600 & & \\
\hline $17.8163(5)$ & 700 & & \\
\hline $16.4130(2)$ & & & 001 \\
\hline $16.5833(8)$ & & & 002 \\
\hline $16.7506(6)$ & & & 003 \\
\hline $16.5316(1)$ & 010 & & \\
\hline $16.792(8)$ & 110 & & \\
\hline $17.0449(5)$ & 210 & & \\
\hline $17.2958(7)$ & 310 & & \\
\hline $17.5304(2)$ & 410 & & \\
\hline $17.7450(5)$ & 510 & & \\
\hline 16.63914 & & & 020 \\
\hline $16.8145(2)$ & & & 021 \\
\hline $16.9825(1)$ & & & 022 \\
\hline $17.1428(4)$ & & & 023 \\
\hline $17.3123(5)$ & & & 024 \\
\hline $17.4631(5)$ & & & 025 \\
\hline
\end{tabular}


TABLE V. continued

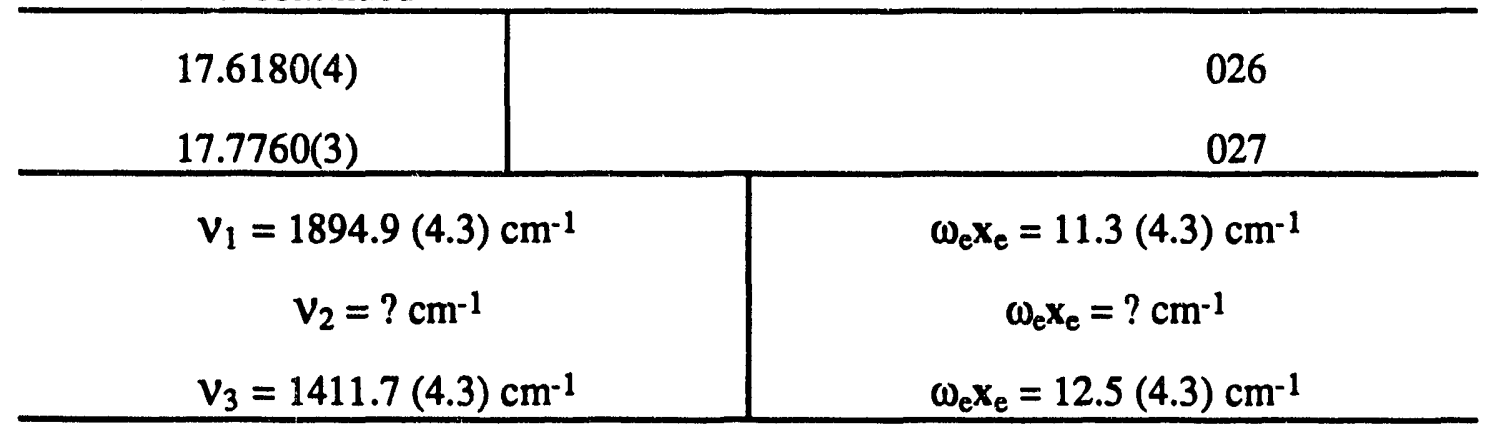

a. See table II. footnotes, also see main text for discussions.

$\mathrm{IE}^{\mathrm{a}} \mathrm{D}_{2} \mathrm{CO}^{+}(\mathrm{eV})$

16.4350(200)

$16.5036(5)$

16.6973(4)

$16.8863(2)$

$17.0799(9)$

$17.2524(2)$

$17.4272(1)$

$17.5972(7)$

16.6341(6)

$16.7310(9)$

$16.8680(2)$

$16.9938(3)$

$17.1300(6)$

17.2621(4)

$17.3873(8)$

$17.5216(9)$

$16.8381(5)$
Vibrational Progressions $\left(V_{3} V_{2} V_{3}\right)$

$000(?)$

001

101

201

301

401

501

601

100

110

120

130

140

150

160

170 
TABLE V. continued

\begin{tabular}{l|ll}
\hline $16.9181(5)$ & 021 \\
$17.0322(9)$ & 022 \\
$17.1525(8)$ & 023 & \\
$17.2621(4)$ & 024 & \\
$17.3732(2)$ & 025 & \\
$17.4909(8)$ & 026 & $\omega_{\mathrm{e}} x_{\mathrm{e}}=22.3(4.3) \mathrm{cm}^{-1}$ \\
$17.6086(7)$ & 027 & $\omega_{\mathrm{e}} \mathrm{x}_{\mathrm{e}}=-12.7(4.3) \mathrm{cm}^{-1}$ \\
\hline$v_{1}=1604.4(4.3) \mathrm{cm}^{-1}$ & $\omega_{\mathrm{e}} \mathrm{x}_{\mathrm{e}}=-11.8(4.3) \mathrm{cm}^{-1}$ \\
\hline
\end{tabular}

a. See footnote of table II., also see main text for discussions. 


\section{Figure Captions:}

Figure 1. The full spectrum of formaldehyde with a resolution of $11 \mathrm{meV}$ FWHM, the result of four sequential scans each lasting about two hours. Each scan was repeated four times to enhance statistics. The designation of the ionic states assumes that the ions have $\mathrm{C}_{2 \mathrm{~V}}$ symmetry.

Figure 2. The full spectrum of $D_{2}$ formaldehyde with a resolution of $11 \mathrm{meV} F W H M$, the result of four sequential scans each lasting about two hours. Each scan was repeated four times to enhance statistics. The designation of the ionic states assumes that the ions have $\mathrm{C}_{2 \mathrm{~V}}$ symmetry.

Figure 3. The photoelectron spectrum of the $\tilde{\mathrm{X}} 1^{2} \mathrm{~B}_{2}$ state of the $\mathrm{H}_{2} \mathrm{CO}^{+}$, with a resolution of $11 \mathrm{meV}$ FWHM. The vibrational progressions are labeled according to the $C_{2} v$ geometry and ${ }_{0}^{1}$ stands for the transition of

$$
M^{+}\left(V_{2}=1\right)+e^{-} \leftarrow M\left(V_{2}=0\right)+\hbar \omega
$$

following standard spectroscopic notations.

Figure 4. The photoelectron spectrum of the $\tilde{\mathrm{X}} 1^{2} \mathrm{~B}_{2}$ state of the $\mathrm{D}_{2} \mathrm{CO}^{+}$, with a resolution of $11 \mathrm{meV}$ FWHM. The vibrational progressions are labeled under the $C_{2} v$ geometry, and notations are the same as in the $\widetilde{X} 1^{2} B_{2}$ state of $\mathrm{H}_{2} \mathrm{CO}^{+}$.

Figure 5. The vibrational autocorrelation function of the $\tilde{\mathrm{X}} 1^{2} \mathrm{~B}_{2}$ state of $\mathrm{H}_{2} \mathrm{CO}^{+}$ after all the corrections have been made. The complex beat paten of the correlation function was the result of excitation of three totally symmetric vibrations, and possibly of the out-of-plane bending vibration as well. The overall slow decay of the correlation function, however, indicates that the 
molecular ions are in a fairly stable configuration.

Figure 6. The vibrational correlation function for the $\widetilde{\mathrm{X}} 1^{2} \mathrm{~B}_{2}$ state of $\mathrm{D}_{2} \mathrm{CO}+$ after all the corrections have been made. The main features of the correlation function are the same as those in the $\tilde{\mathrm{X}} 1^{2} \mathrm{~B}_{2}$ state of $\mathrm{H}_{2} \mathrm{CO}+$.

Figure 7. The photoelectron spectrum of the $\tilde{\mathrm{A}}^{2} \mathrm{~B}_{1}$ state of $\mathrm{H}_{2} \mathrm{CO}^{+}$with a resolution of $11 \mathrm{meV}$ FWHM. The vibrational progressions are assigned assuming the molecular ions have $\mathrm{C}_{2 \mathrm{~V}}$ equilibrium geometry. Notations are the same as in Figure 3. Rotational cooling combined with the high resolution obtained by this study, enabled the multiplet vibrational fine structures to be fully resolved here for the first time.

Figure 8. The photoelectron spectrum of the $\tilde{\mathrm{A}}^{2} \mathrm{~B}_{1}$ state of $\mathrm{D}_{2} \mathrm{CO}^{+}$with a resolution of $11 \mathrm{meV}$ FWHM. The vibrational progressions are assigned assuming the molecular ions have $\mathrm{C}_{2}$ v equilibrium geometry. Notations are the same as in Figure 3. Note that the vibrational progressions are different than those of $\mathrm{H}_{2} \mathrm{CO}^{+}$.

Figure 9. The vibrational autocorrelation function calculated for the $\tilde{\mathrm{A}}^{2} \mathrm{~B}_{1}$ state of $\mathrm{H}_{2} \mathrm{CO}^{+}$. Note the beat pattem caused by multimode excitation and a small spin-orbit splitting for this state. Shown at the bottom, is the correlation function after corrections have been made. The monotonic decay of the correlation function indicates a stable configuration in the Franck-Condon region.

Figure 10. The same as in Figure 9, for the $\tilde{\mathrm{A}}^{2} \mathrm{~B}_{1}$ state of $\mathrm{D}_{2} \mathrm{CO}^{+}$. Note the isotope effects at play in the correlation function. The correlation function of $\mathrm{D}_{2} \mathrm{CO}^{+}$ 
decays much slower than $\mathrm{H}_{2} \mathrm{CO}^{+}$.

Figure 11. The photoelectron spectrum of the $\widetilde{\mathrm{B}}{ }^{2} \mathrm{~A}_{1}$ and $\widetilde{\mathrm{C}}{ }^{2} \mathrm{~B}_{2}$ states of $\mathrm{H}_{2} \mathrm{CO}^{+}$with a resolution of $11 \mathrm{meV}$ FWHM. Note the FWHM of the peaks in this band are only about $12 \mathrm{meV}$, indicating very effective rotational relaxation in the supersonic expansion. The features clearly show that there is only one vibrational progression in the $\widetilde{B}{ }^{2} A_{1}$ state

Figure 12. The photoelectron spectrum of the $\widetilde{\mathrm{B}}{ }^{2} \mathrm{~A}_{1}$ state of $\mathrm{D}_{2} \mathrm{CO}+$ with a resolution of $11 \mathrm{meV}$ FWHM. Note the FWHM of the peaks in this band are only about $12 \mathrm{meV}$, indicating very effective rotational relaxation in the supersonic expansion. The features observed clearly show that there are at least two vibrational modes excited.

Figure 13. The vibrational correlation function calculated for the $\widetilde{\mathrm{B}}{ }^{2} \mathrm{~A}_{1}$ state of $\mathrm{H}_{2} \mathrm{CO}^{+}$ after all corrections were made. The correlation function clearly shows that there is only one vibrational mode excited. The slow decay of the correlation function ard the ability of the correlation function to remain large for a sufficient long time indicates again, that the ionic state is very stable with respect to deformations along the $\mathrm{Q}_{2}$ normal coordinate.

Figure 14. The vibrational correlation function calculated for the $\widetilde{\mathrm{B}}{ }^{2} \mathrm{~A}_{1}$ state of $\mathrm{D}_{2} \mathrm{CO}^{+}$ after corrections for rotation and instrumental response function. Note the slow modulations on the correlation function which was caused by the excitation of more than one vibrational mode. The complexity of this beat pattern makes correction not feásible, and it was not attempted. However, the slowly decay pattern of the correlation function is still visible. 
Figure 15. The photoelectron spectrum of the $\widetilde{\mathrm{C}}{ }^{2} \mathrm{~B}_{2}$ state of $\mathrm{D}_{2} \mathrm{CO}^{+}$with a resolution of $11 \mathrm{meV}$ FWHM. Even with very effective rotational cooling and current high resolution measurements, the complexity of this band still eludes our analysis. The band structure observed is the characteristic of a repulsive state.

Figure 16. The vibrational correlation function calculated for the $\widetilde{C} 2^{2} B_{2}$ state of $\mathrm{H}_{2} \mathrm{CO}^{+}$after all corrections were made. The main feature of the correlation function is the extremely fast decay and loss of correlation, indicating ultra fast intramolecular dynamics processes.

Figure 17. The vibrational correlation function calculated for the $\widetilde{\mathrm{C}} 2^{2} \mathrm{~B}_{2}$ state of $\mathrm{D}_{2} \mathrm{CO}^{+}$after all corrections were made. The main feature of the correlation function is the extremely fast decay and loss of correlation. Just as in most other states studied here, the isotope effect does show up in the decay of the correlation function. 


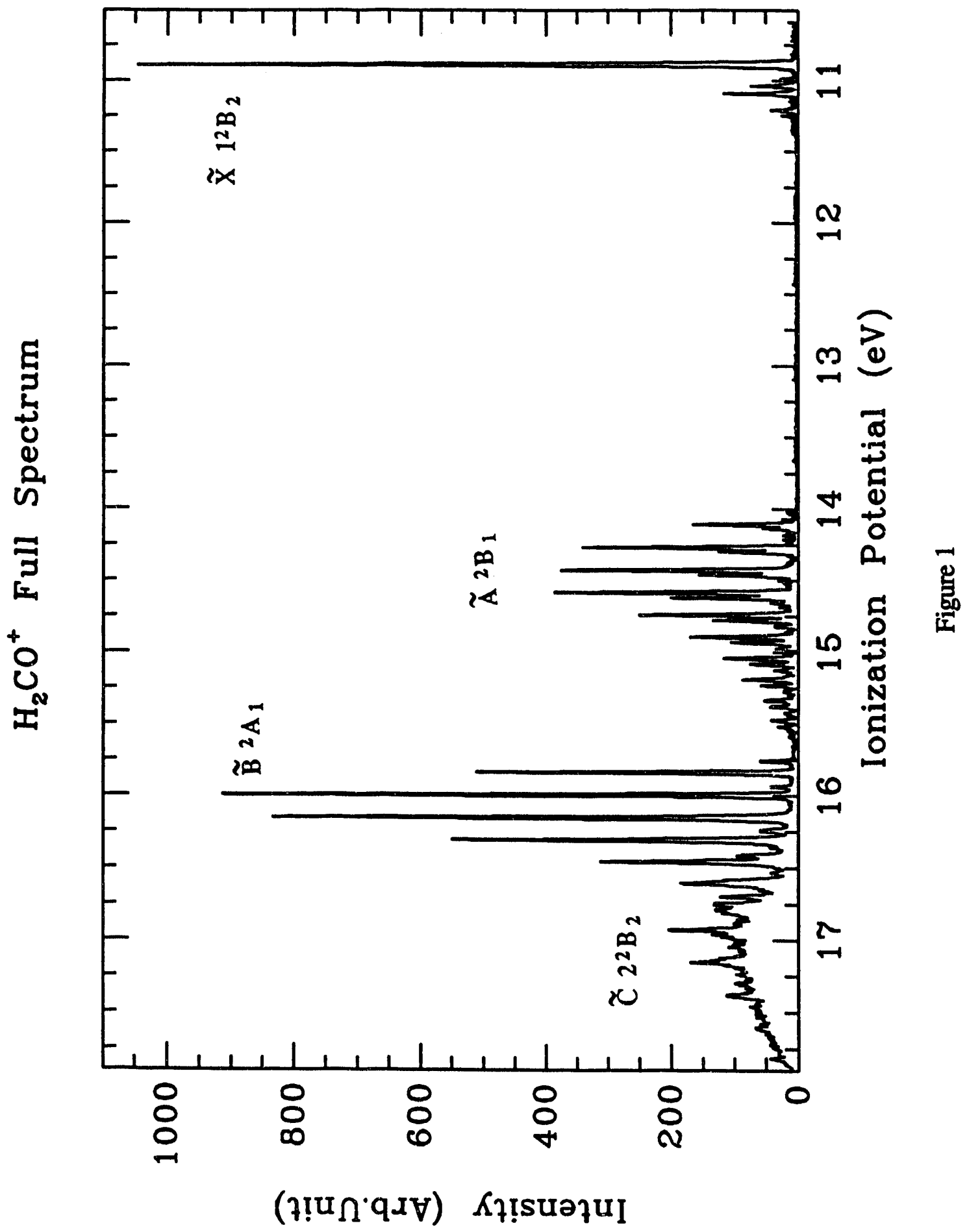




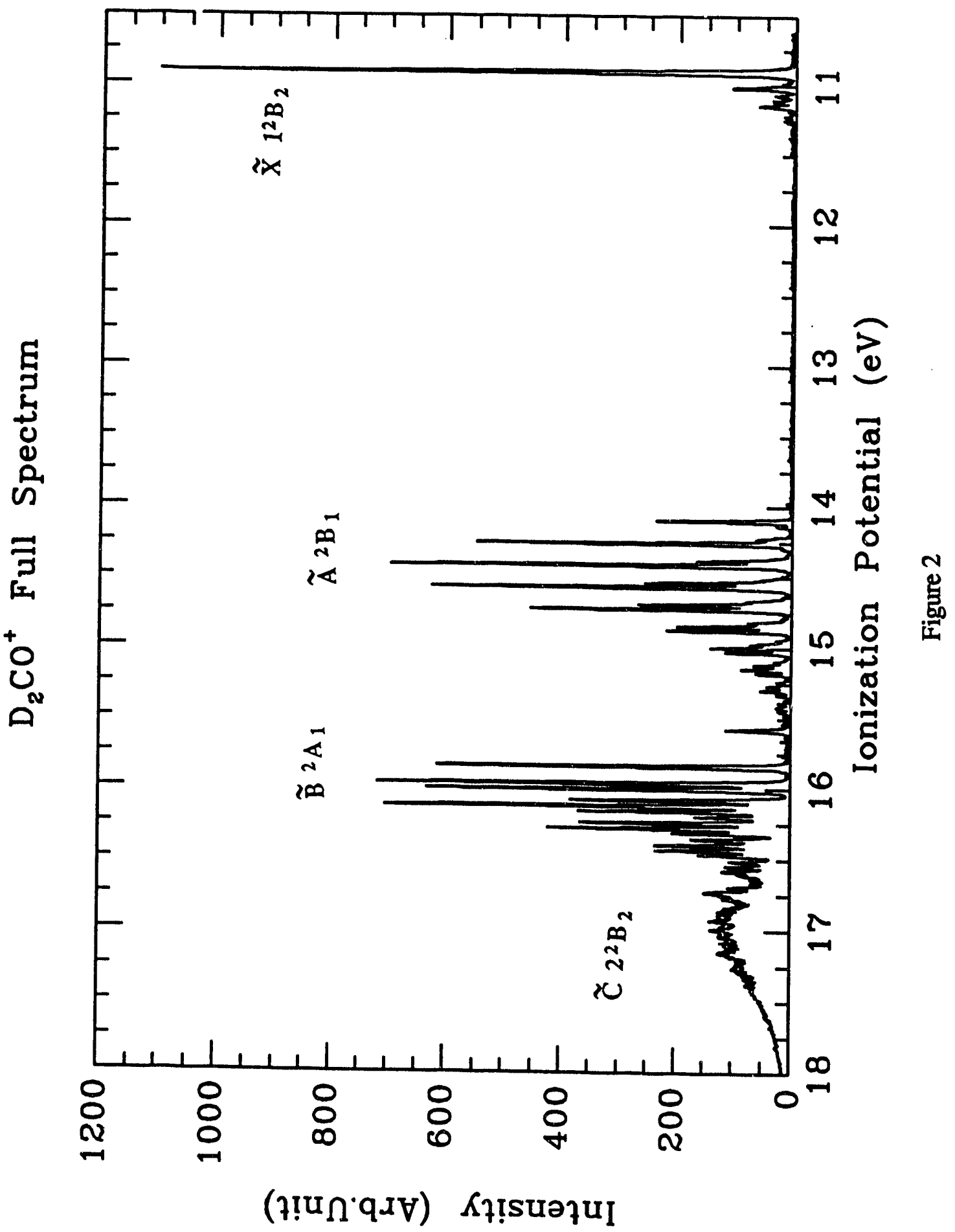




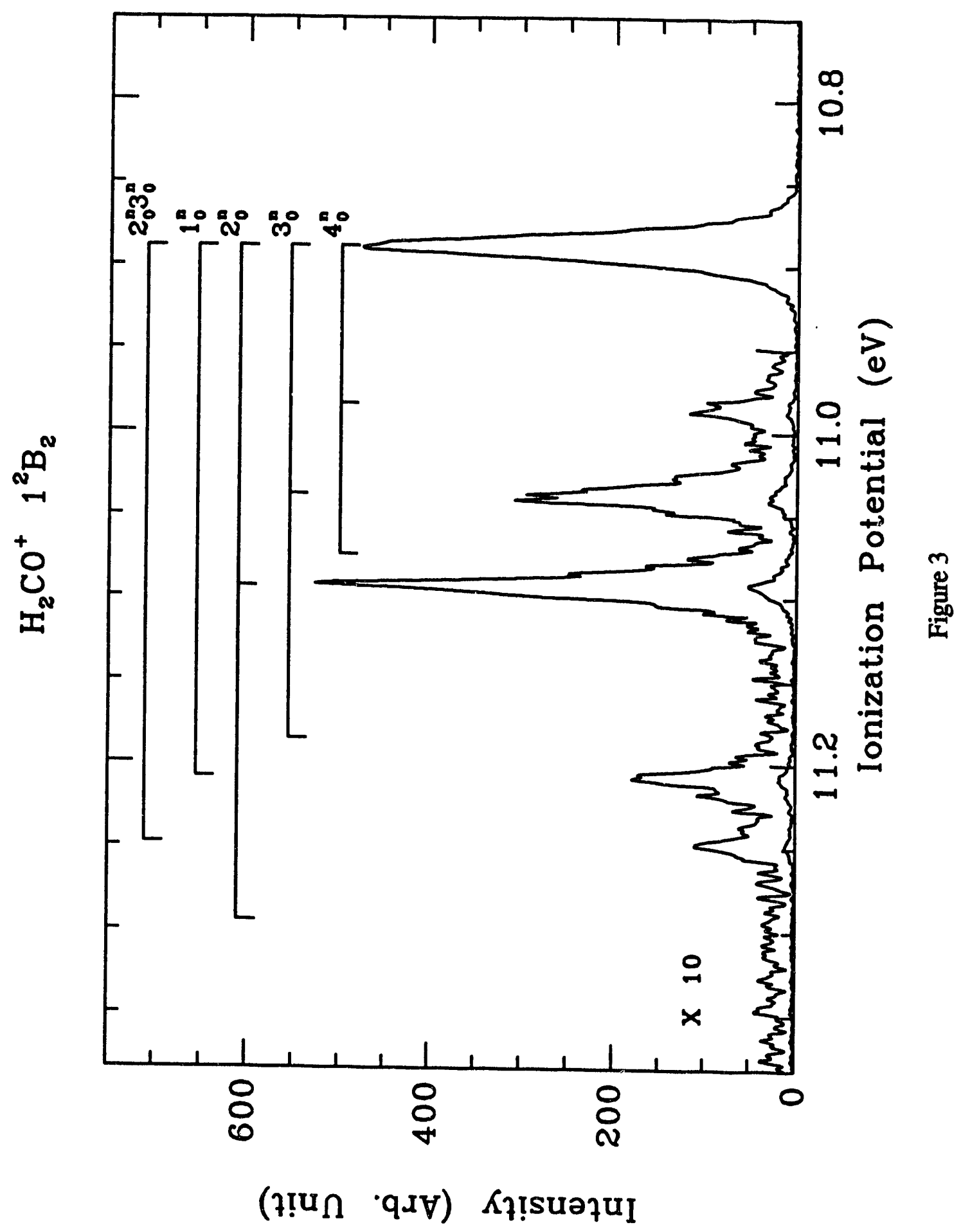




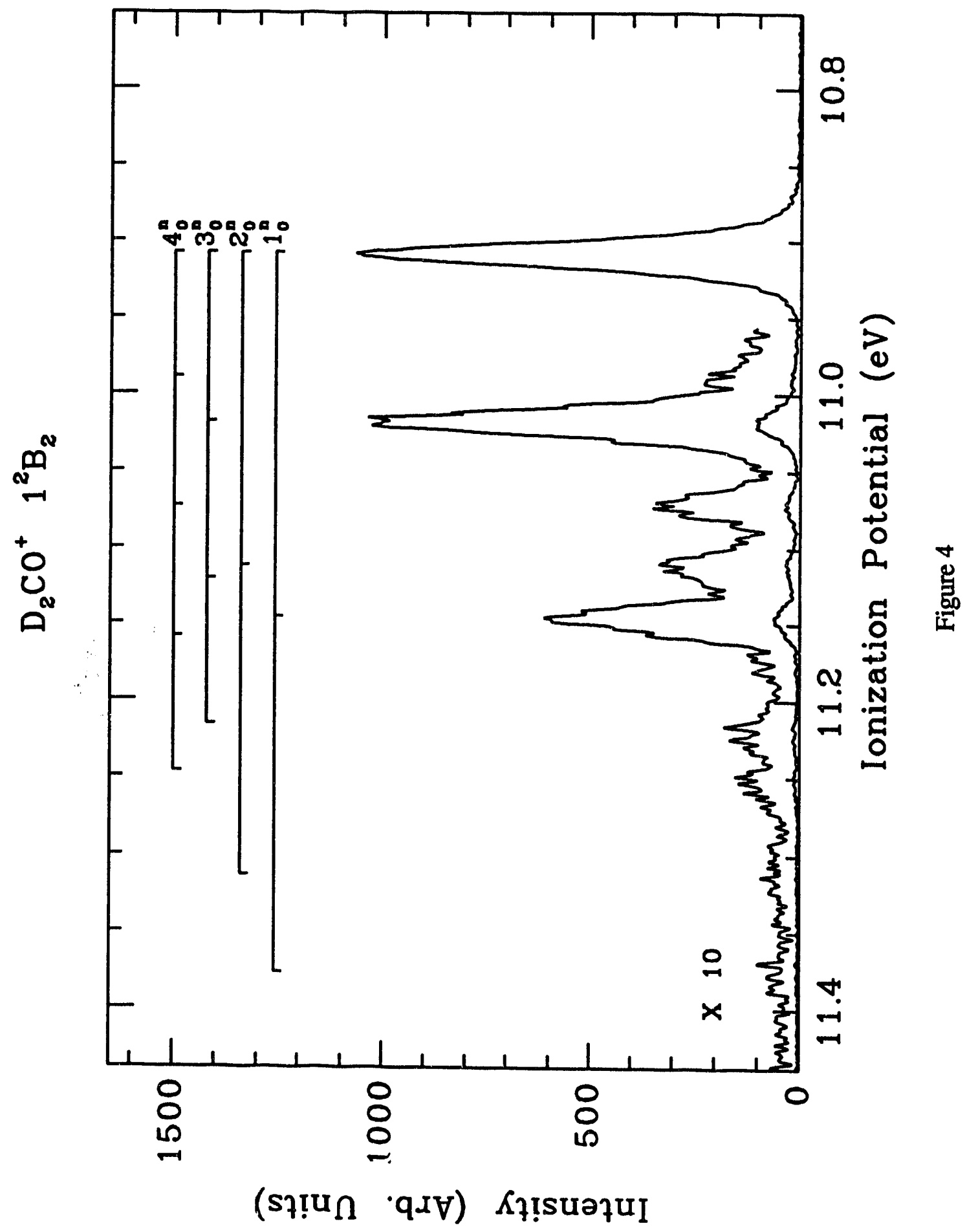




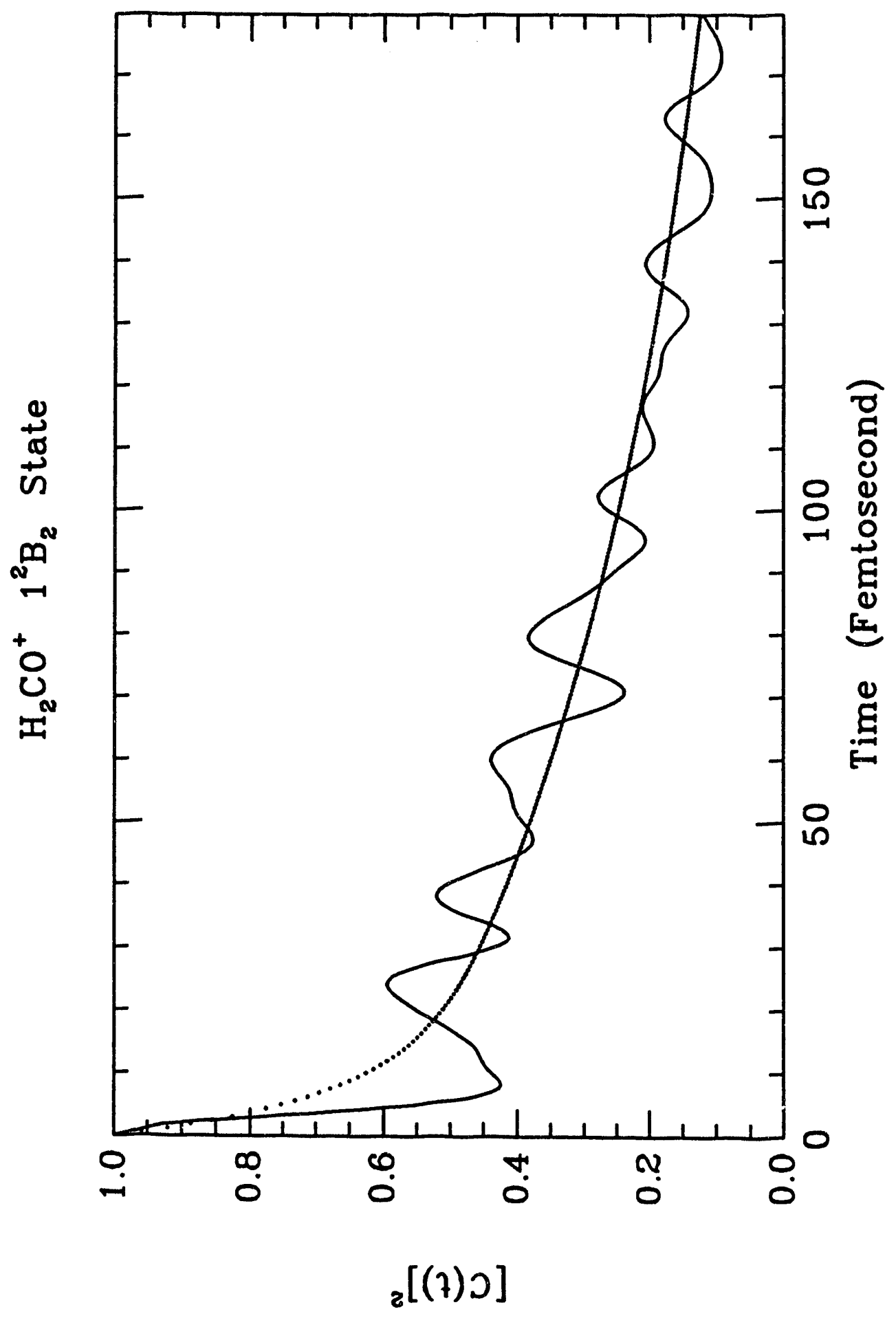

号 


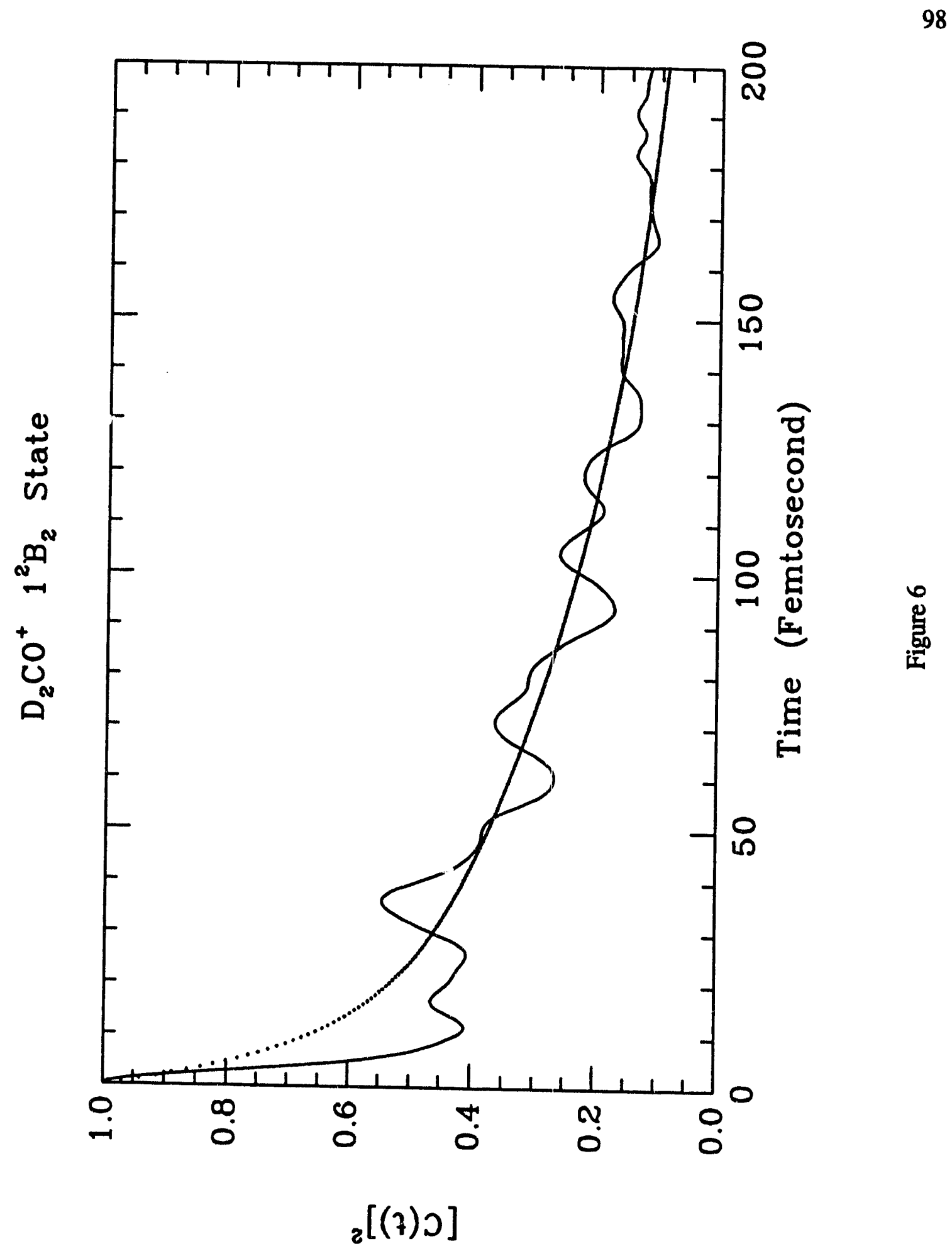




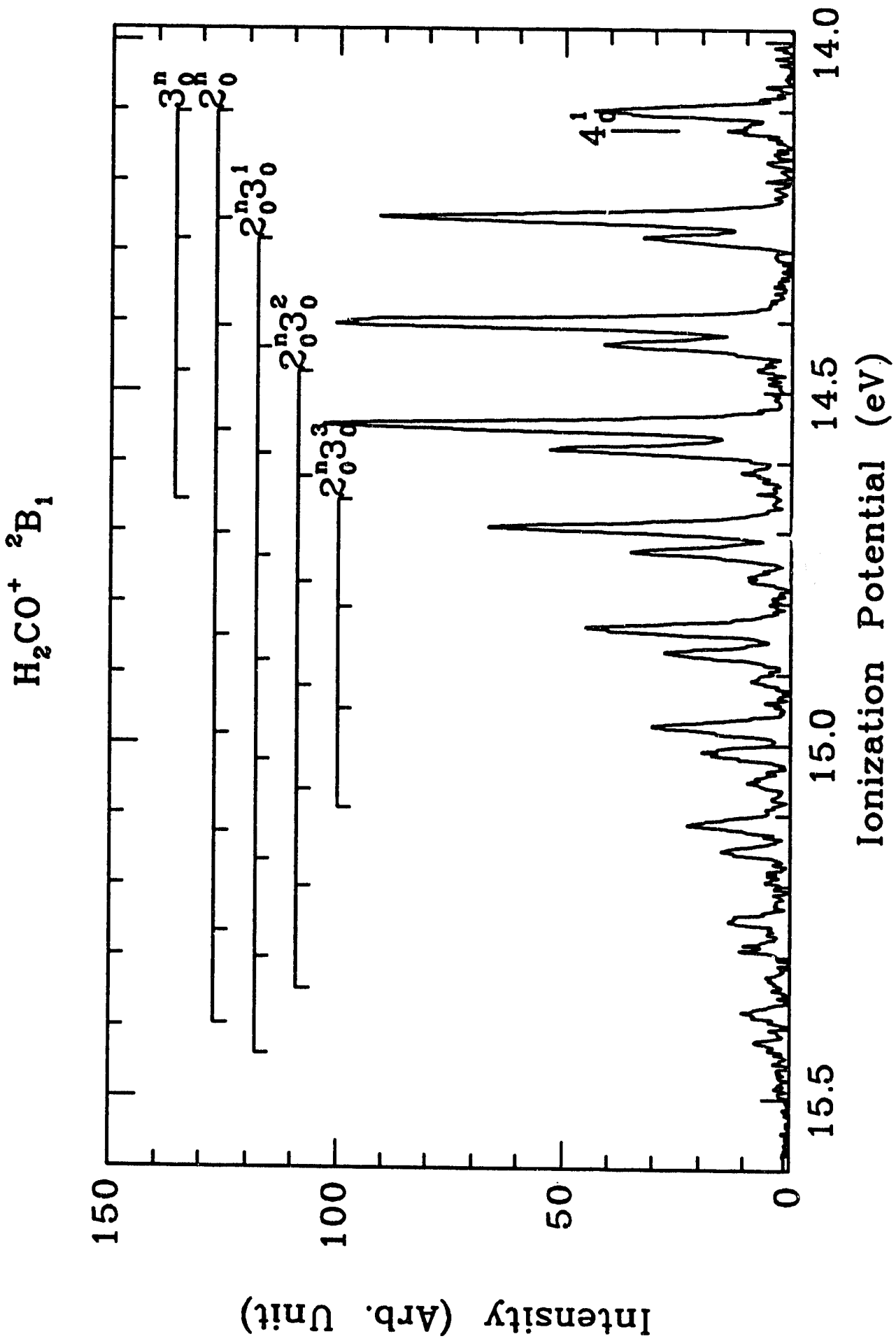

告 


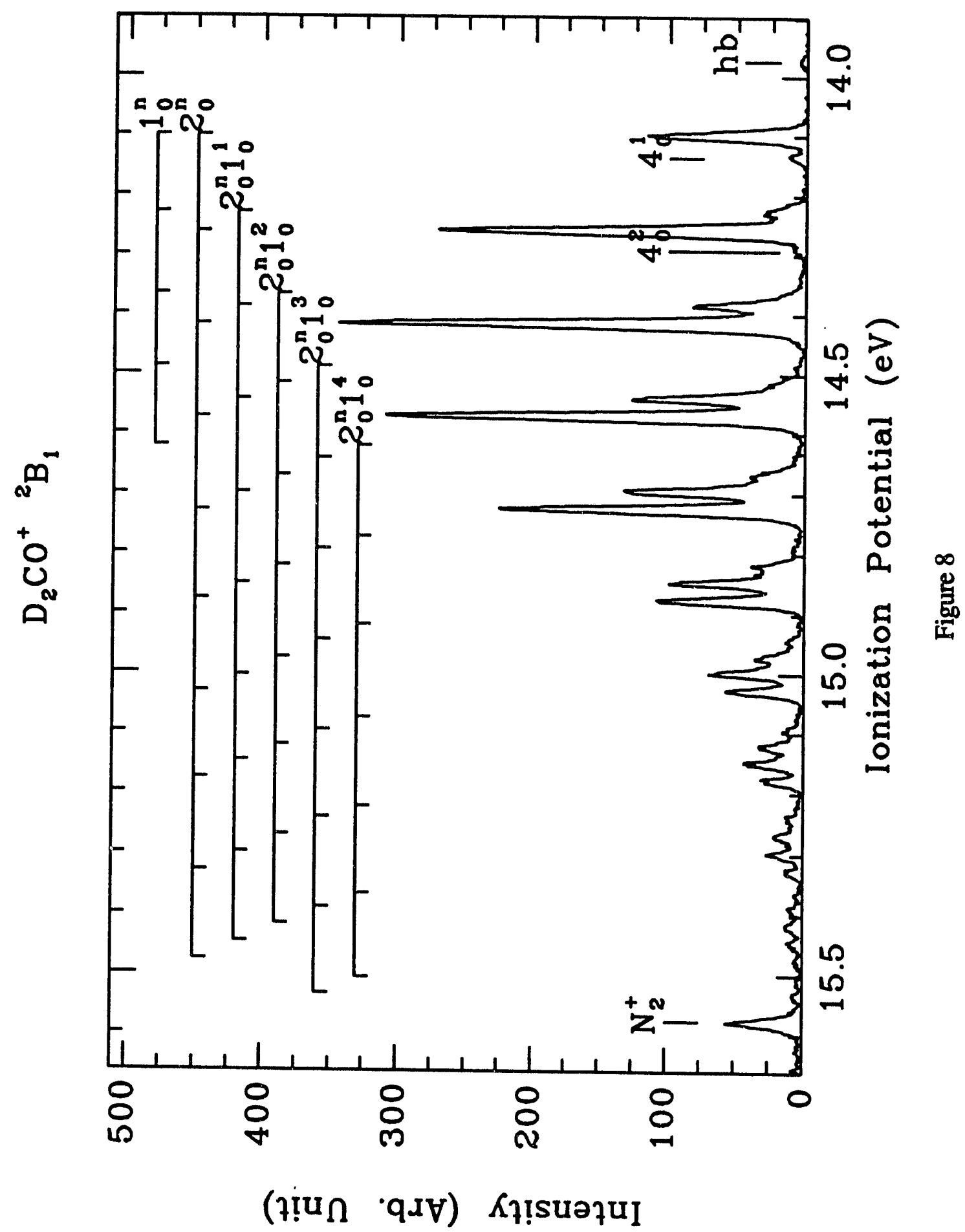




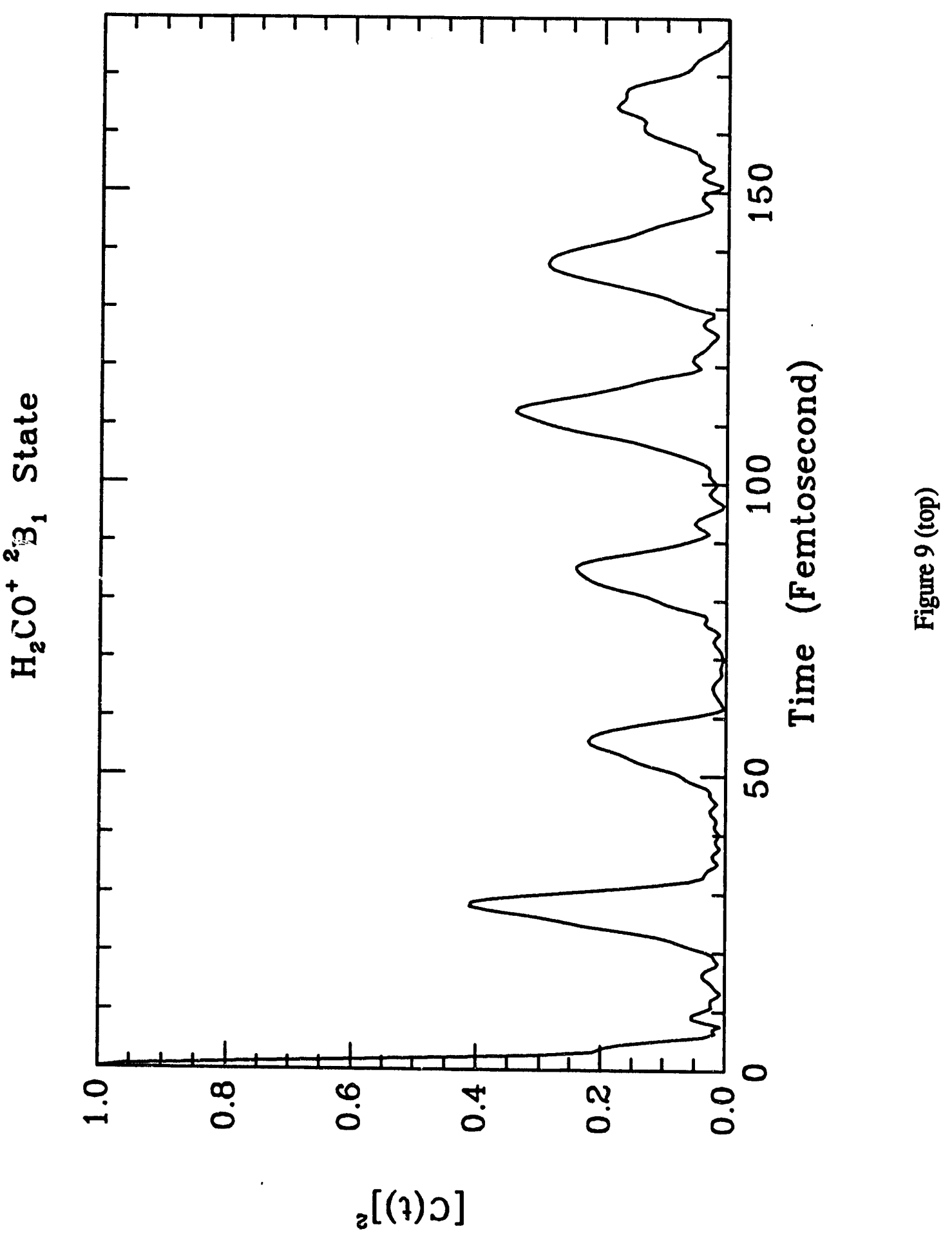




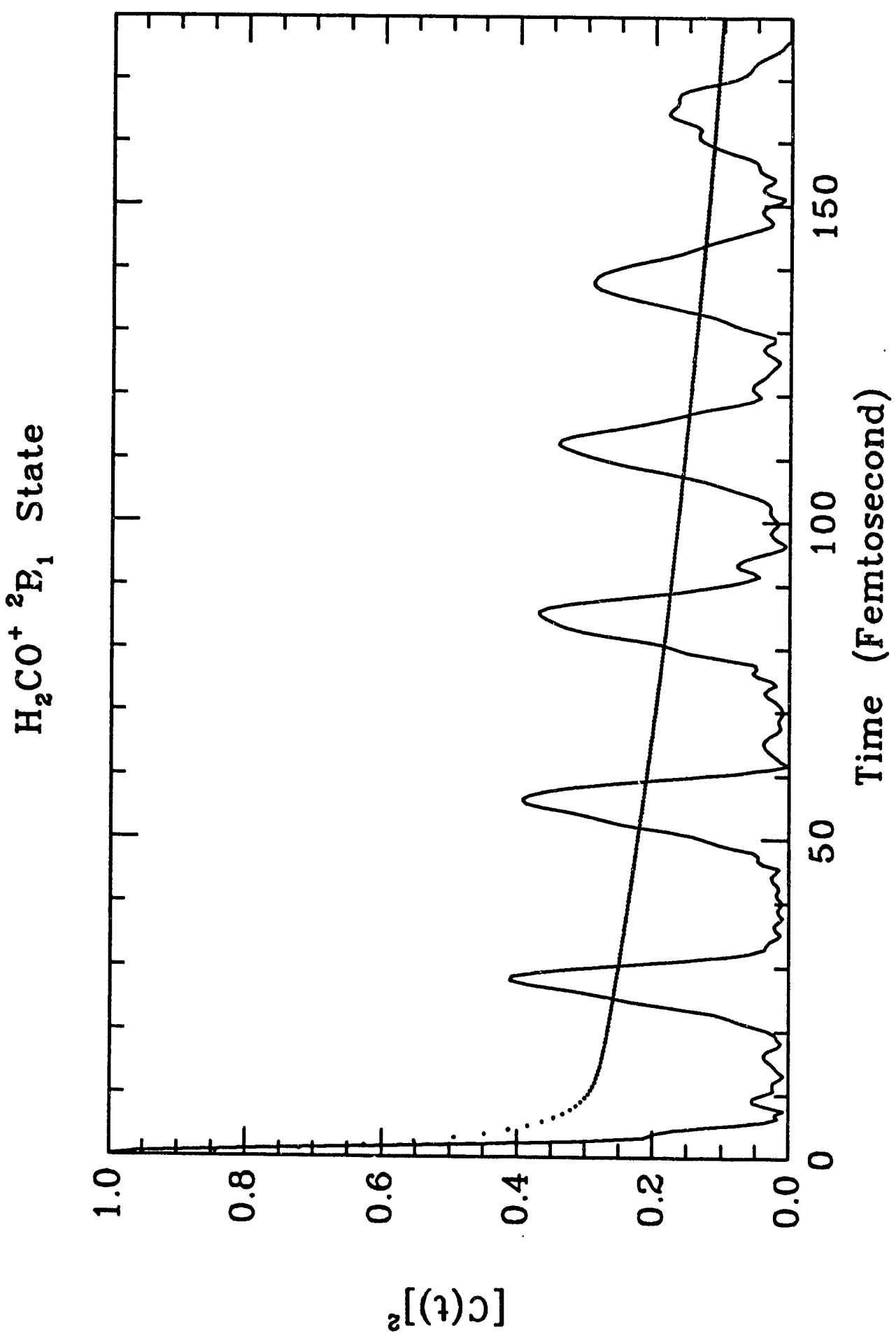

톨 


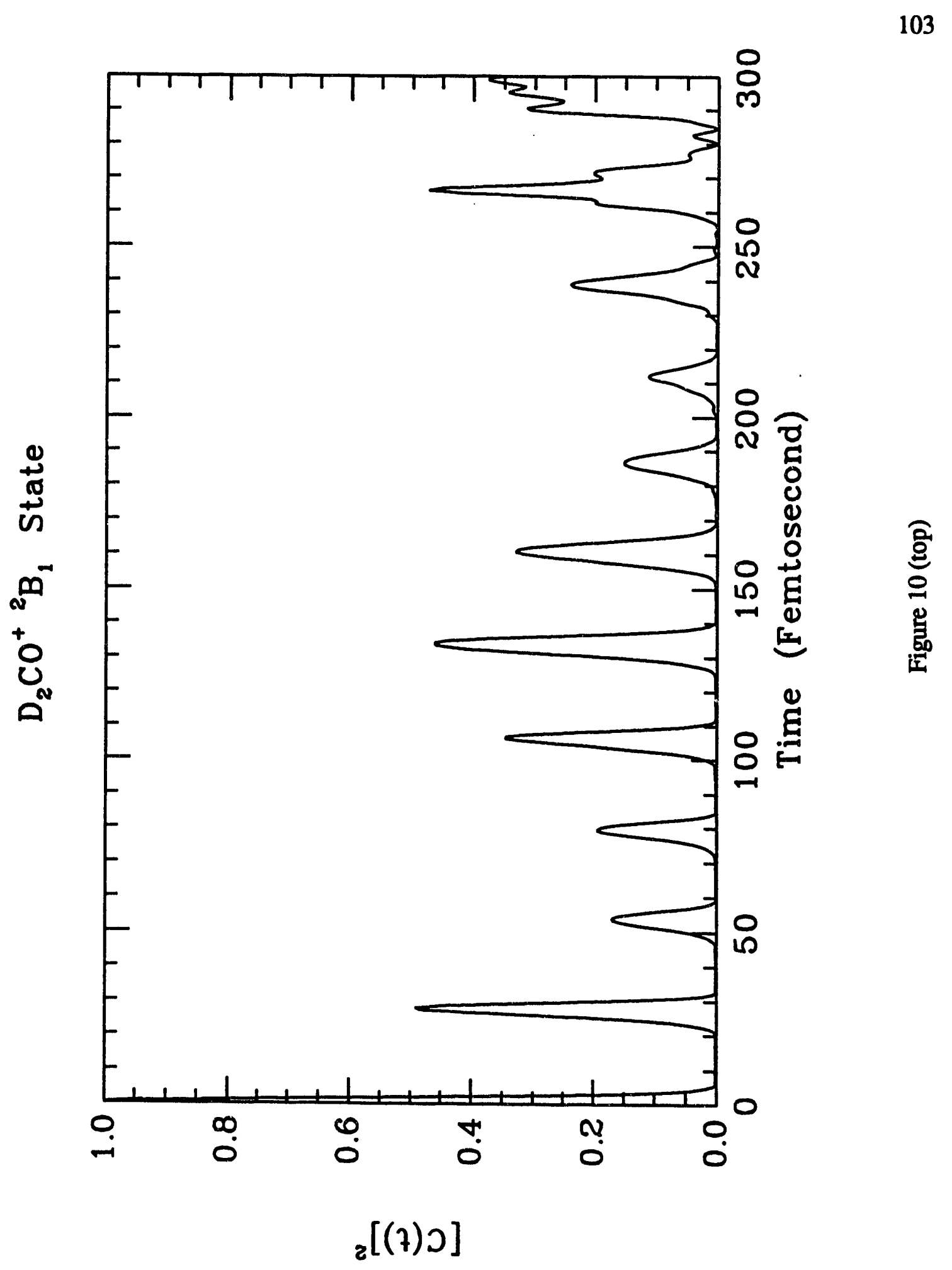




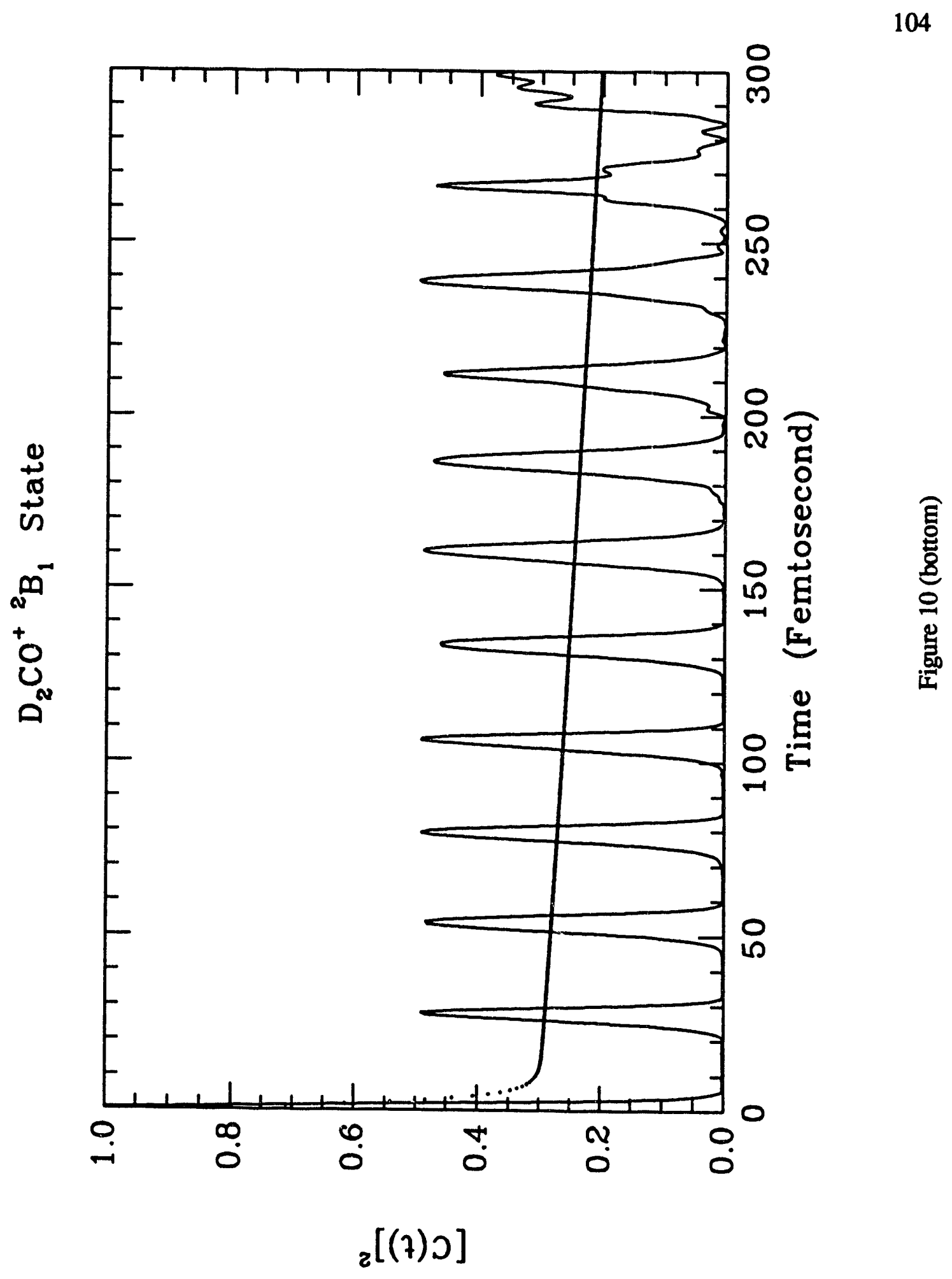




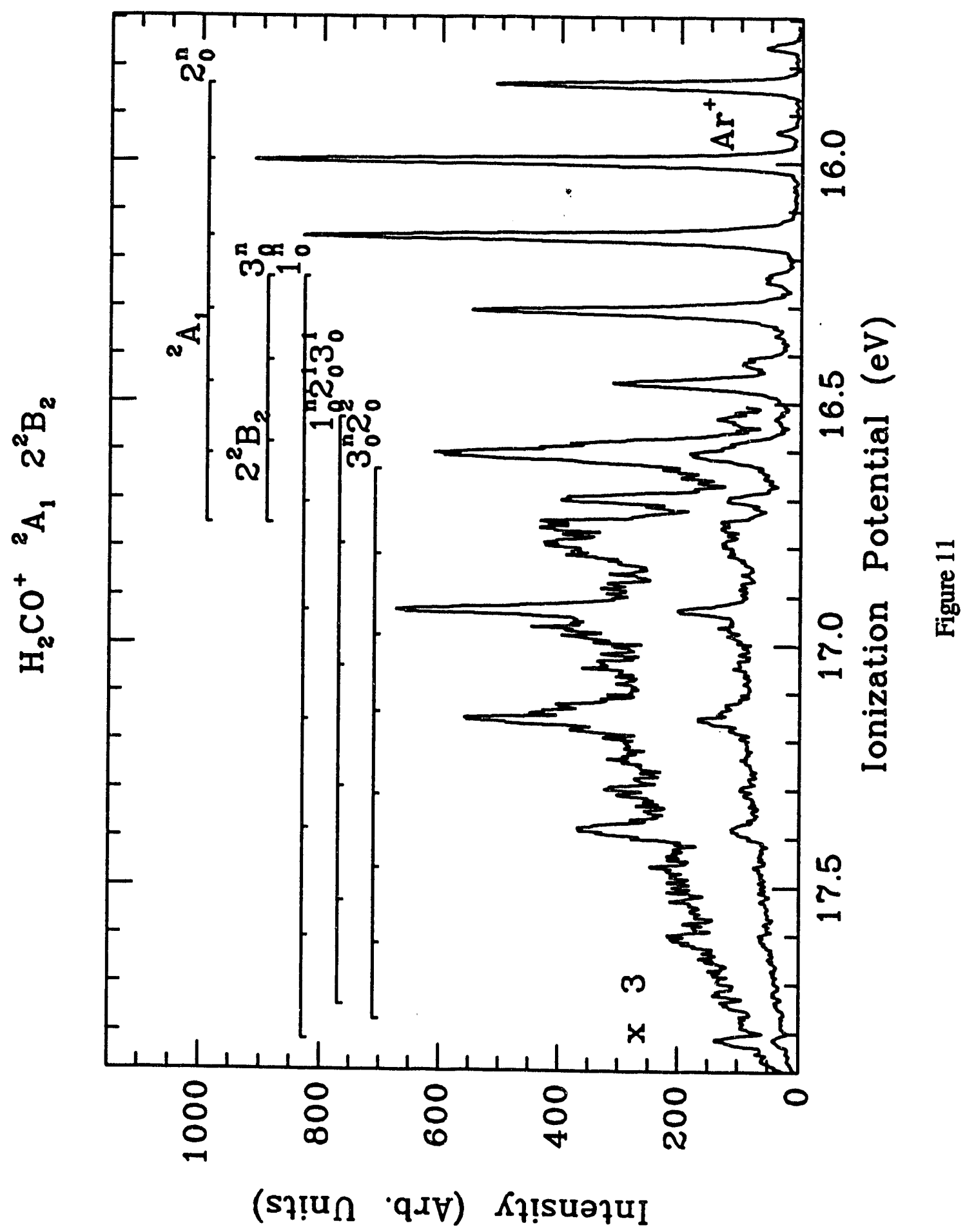




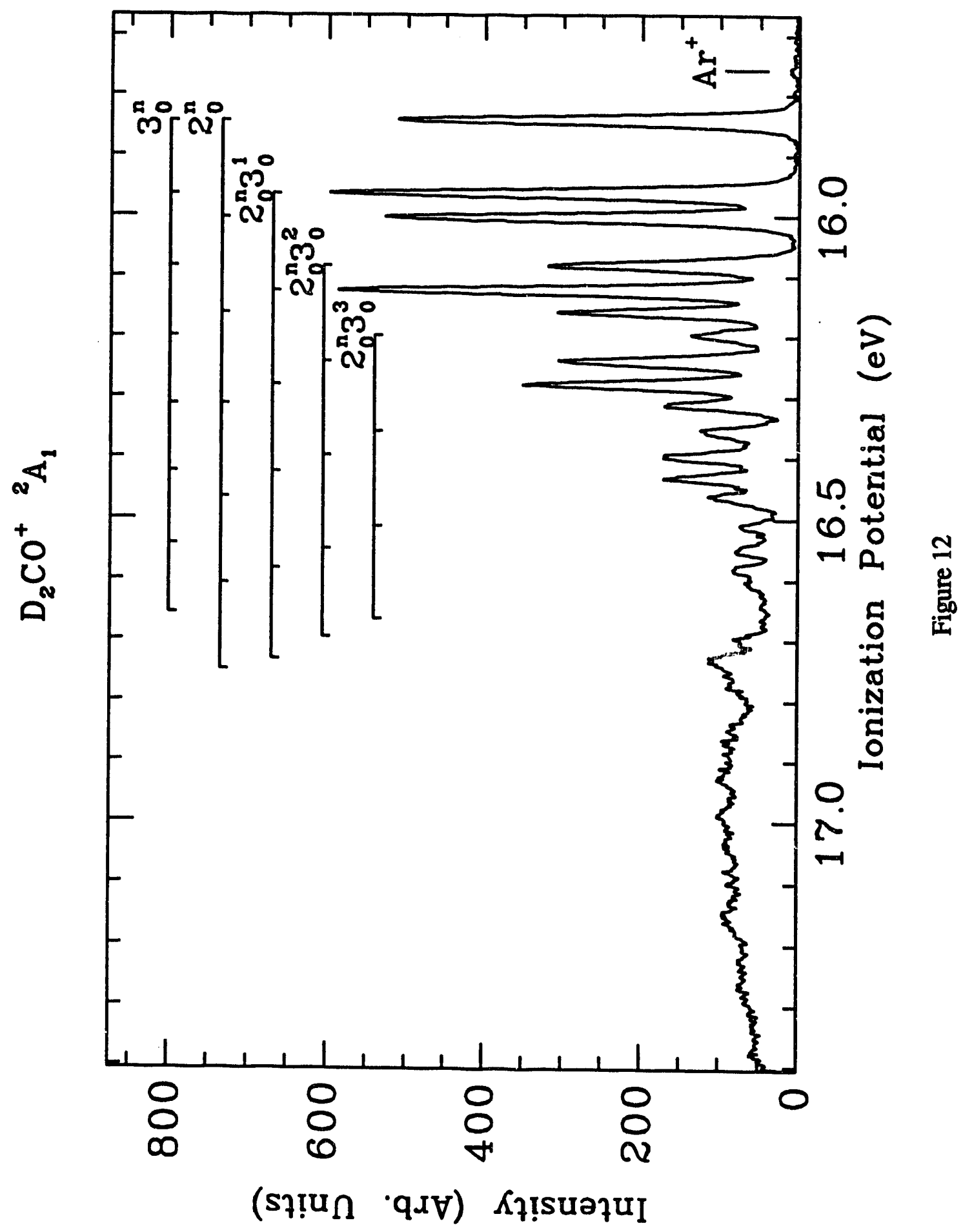




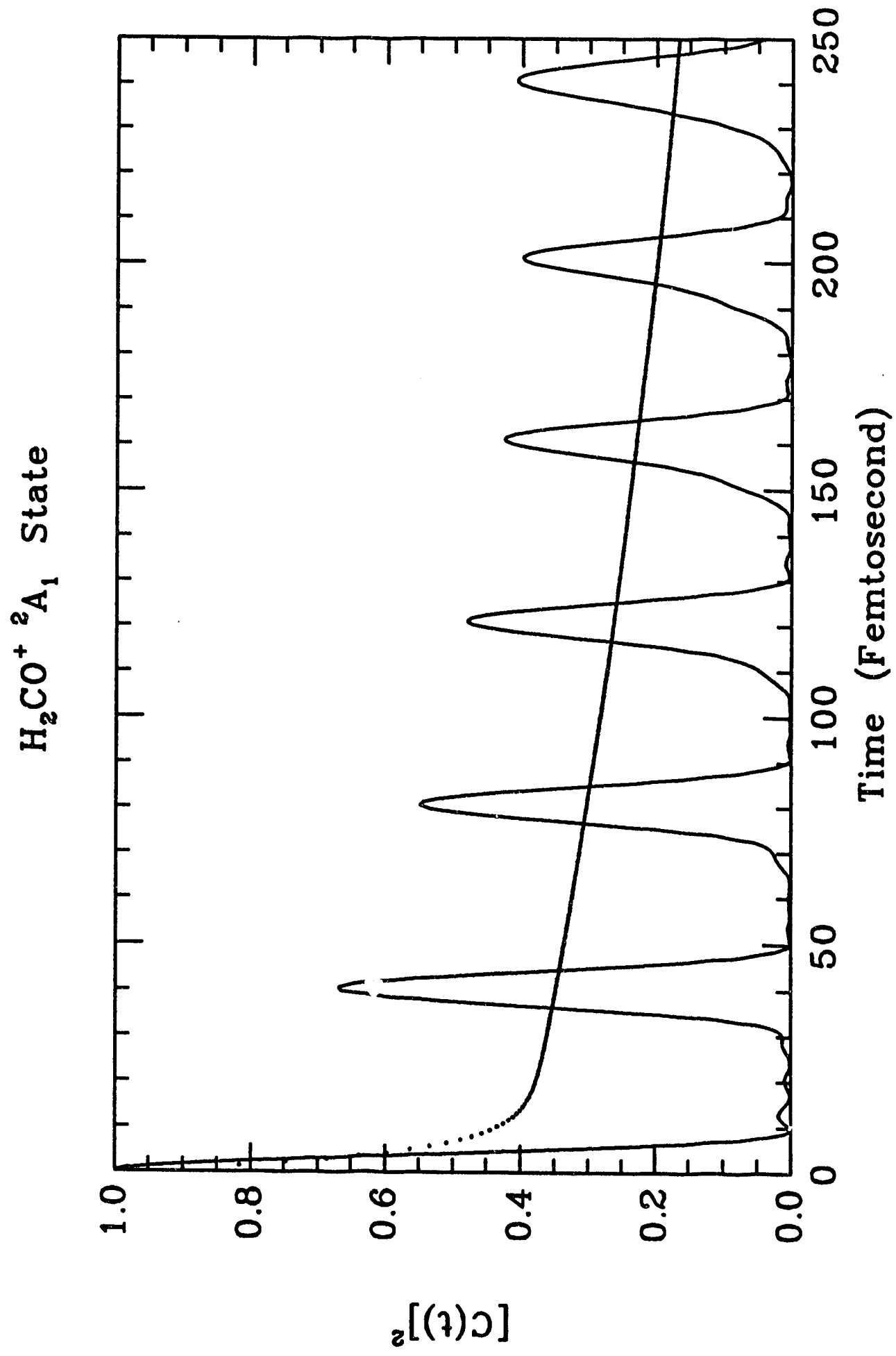

䍃 


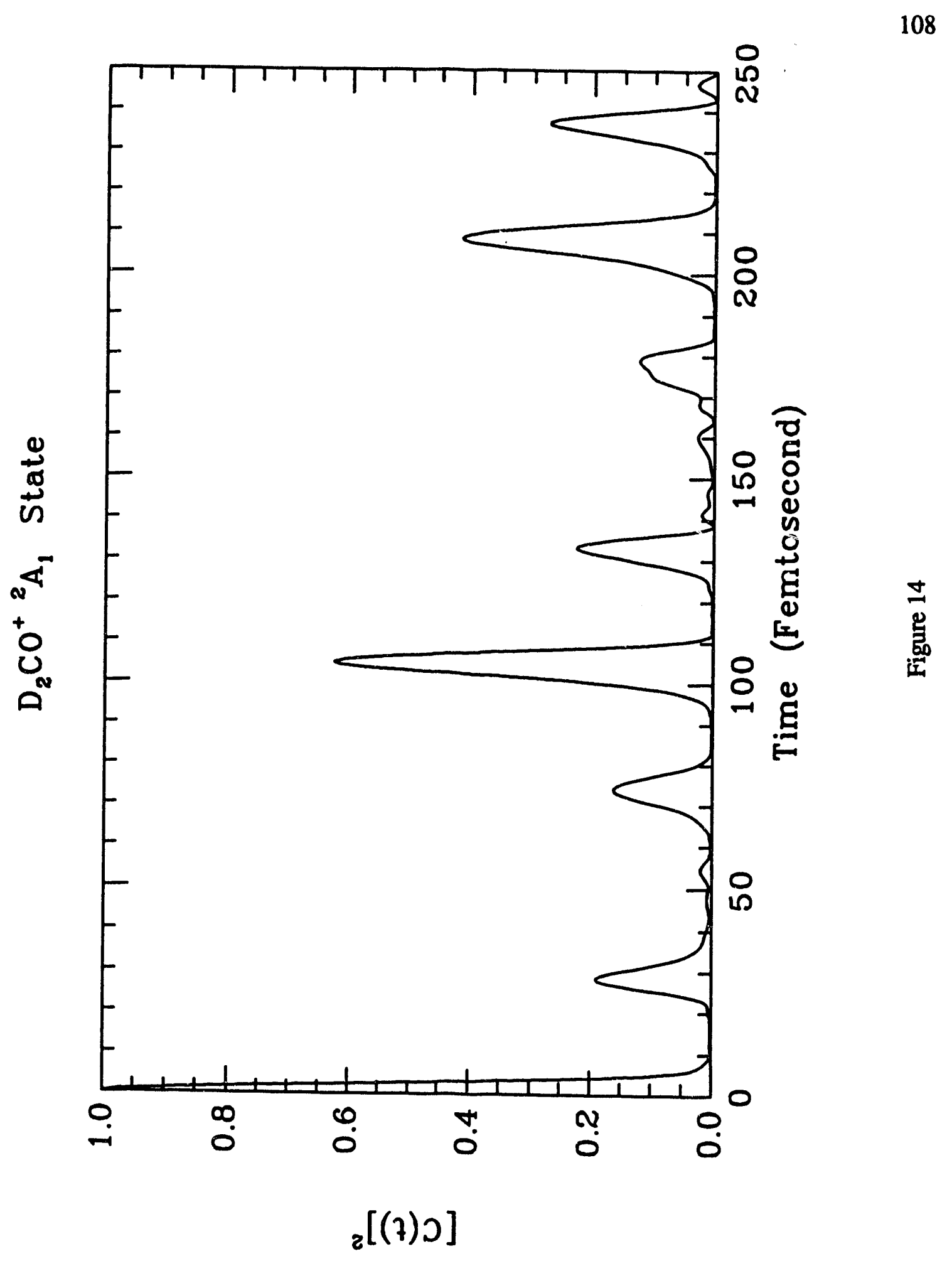




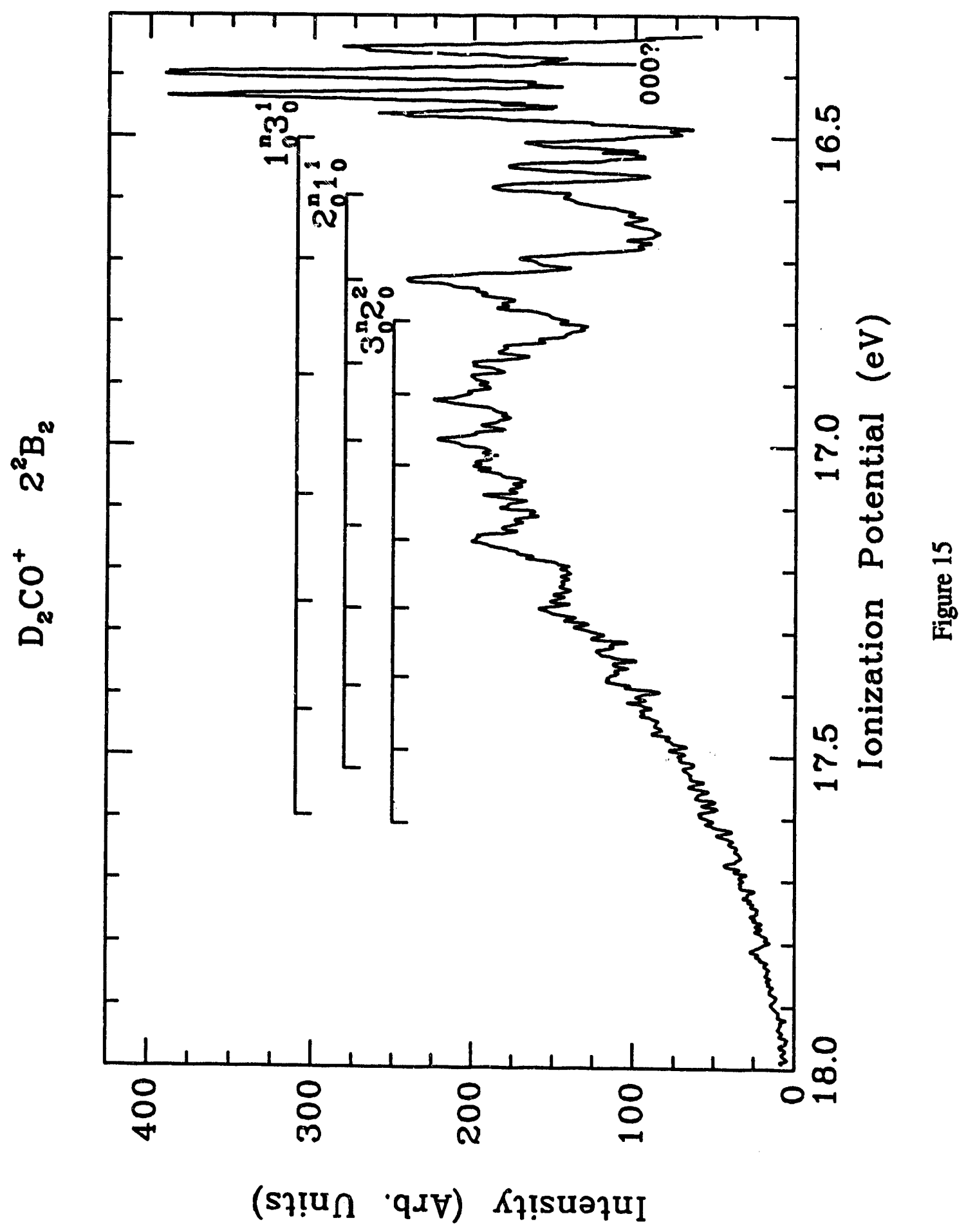




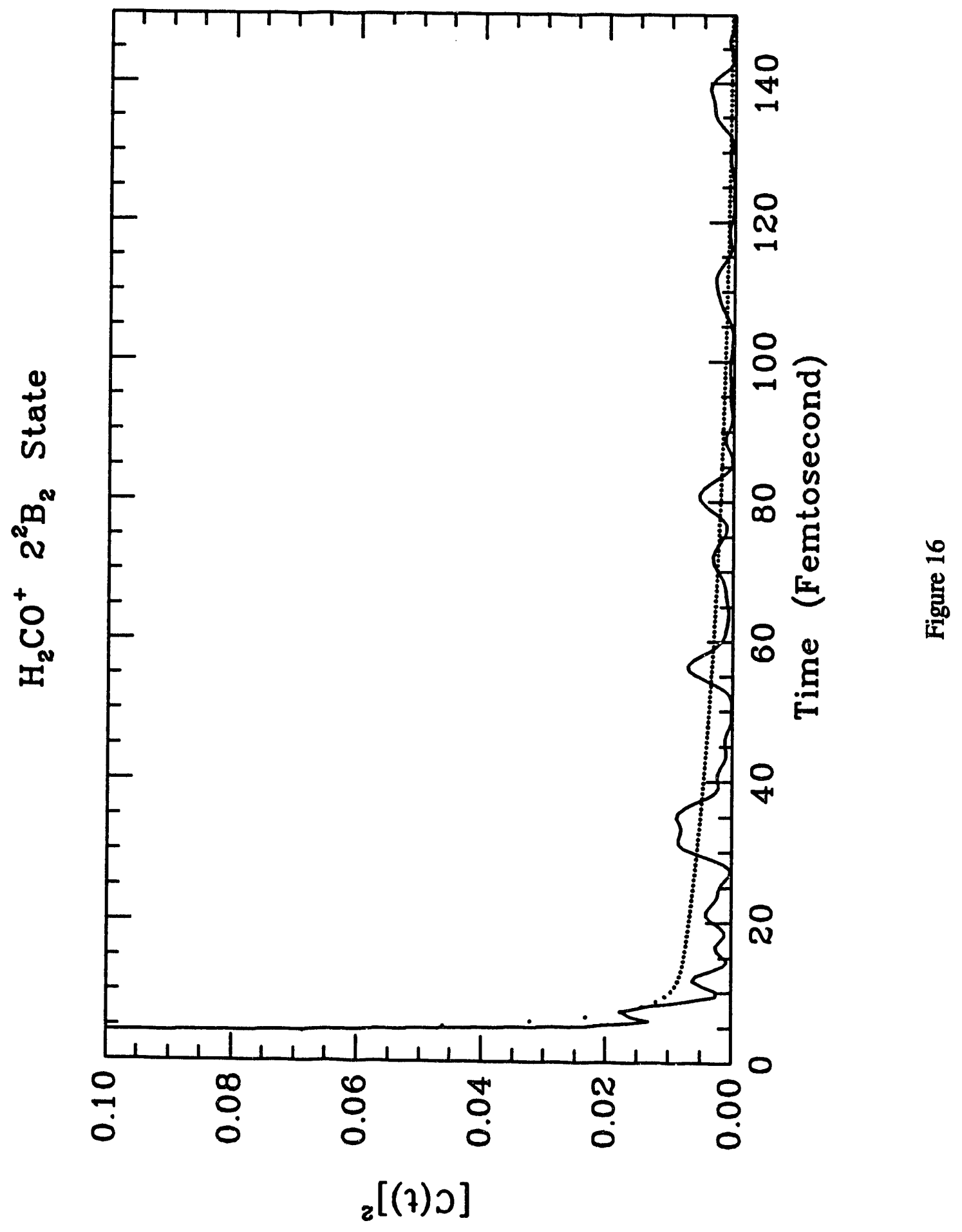




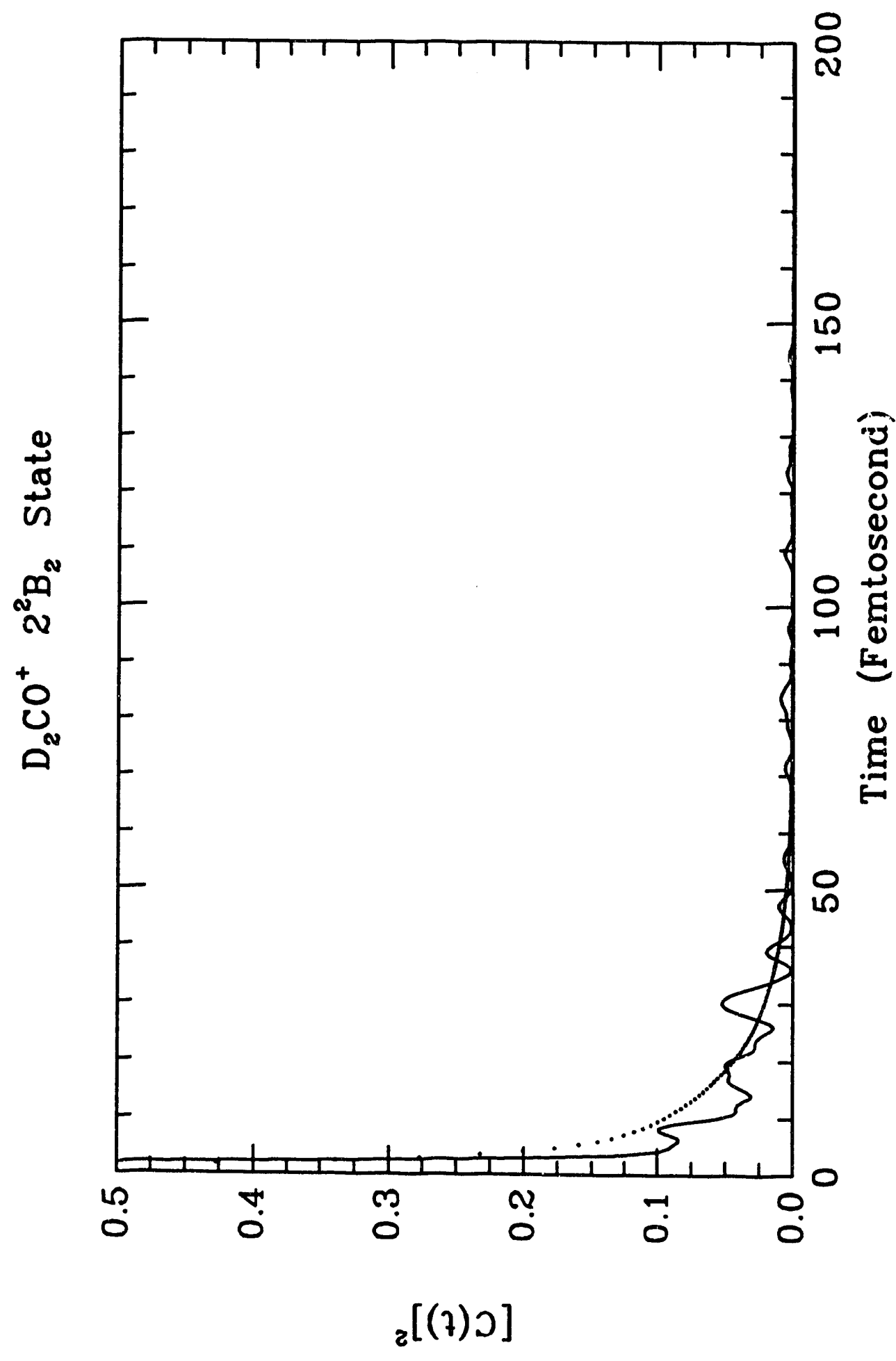

111

可 


\section{Chapter Four: High Resolution Photoelectron Spectroscopy and Femtosecond Intramolecular Dynamics of $\mathrm{H}_{2} \mathrm{CCO}^{+}$and $\mathrm{D}_{2} \mathrm{CCO}^{+}$}

\section{A. Introduction}

The photoelectron spectra of ketene and deuterated ketene have been the subject of extensive experimental and theoretical studies. ${ }^{1-5}$ Baker et al. ${ }^{1}$ was the first group to obtain the helium I $\alpha(21.21732 \mathrm{eV})$ resonance line photoelectron spectrum of ketene and $D_{2}$-ketene. The vibrational fine structures unveiled in this study were partially explained by the then-available theoretical calculations. Hall et al. ${ }^{4}$ studied the ketene and $D_{2}$-ketene photoelectron spectrum using both helium I $\alpha$ and helium II $\alpha(40.81369 \mathrm{eV})$ resonance lines. The assignments of the adiabatic ionization energies (AIE), the vertical ionization energies (VIE), and some vibrational fine structure analysis were also given. ${ }^{1-5}$ The theoretical works have been largely based on these two experimental studies. Very recently, in an $a b$ initio study of ketene and $D_{2}-k e t e n e$, Takeshita ${ }^{6}$ has given a detailed theoretical analysis on the photoelectron spectra of ketene and $D_{2}$-ketene and the vibrational analysis of the fine structures observed in the experimental studies of Baker and Hall. However, the disparity between the experimental and theoretical results is substantial. Especially in the second band (the ionic first excited $\tilde{A} 1^{2} B_{2}$ state), the sharp onset observed in the photoelectron spectrum can not be reproduced by the theoretical calculation. Hall et al. proposed that the complexity of this band might be due to the predissociation of ketene. Takeshita, on the other hand, showed that ketene cation in the $\tilde{\mathrm{A}} 1^{2} \mathrm{~B}_{2}$ state is stable. He suggested that the complexity might be caused by the superposition of peaks associated with the $\widetilde{\mathrm{X}} 1^{2} \Pi_{\mathrm{g}}$ band of $\mathrm{CO}_{2}^{+}$and with the second excited $\widetilde{\mathrm{B}} 2^{2} \mathrm{~B}_{1}$ state of ketene. The simplicity of the theoretical band compared with the experimental one was attributed to the near degeneracy of vibrations (Fermi resonances), $v_{2} \approx 2 v_{4}$, and 
$V_{2} \approx V_{3}+V_{4}$, and might also be attributed to the inadequacy of the theoretical calculation caused by the neglecting of the anharmonicity and the couplings among the vibrations. There were clearly disagreements between the vibrational progression length observed in the experiments and the theoretical calculations. There are extensive studies 45 on the photochemistry and unimolecular dissociation dynamics on the neutral ketene molecule. The photochemistry and unimolecular dynamics of ketene cations have not attracted much attention so far.

In this work, we report the high resolution (FWHM $11.5 \mathrm{meV}$ ) helium I $\alpha$ photoelectron spectra of $\mathrm{H}_{2} \mathrm{CCO}$ and $\mathrm{D}_{2} \mathrm{CCO}$. Improved resolution and effective cooling of the sample by supersonic expansion enabled us to determine the adiabatic ionization potentials for the states accessible by the helium I $\alpha$ resonance line $(21.21732 \mathrm{eV})$ to a much higher accuracy and to give new interpretations of the vibration structures observed. The results compared very favorably with the very recently ab initio calculation by Takeshita, although, there are clearly scme disagreements between the theoretically calculated spectra and the experimentally observed ones. Using the autocorrelation function formalism first discussed by Heller ${ }^{7-10}$ for electronic photon absorption and emission, later by Lorquet et al. ${ }^{11.14}$ and by Ruscic ${ }^{13}$ for photoelectron spectroscopy, and modified by Pollard et al. ${ }^{15}$ and Reutt et al.; ${ }^{16,17}$ the ferntosecond ultrafast intramolecular dynamics of the ketene cation in its various ionic states is discussed.

The details of the experiments are described in section B of this chapter. The vibronic coupling and isotope effects on vibronic coupling are discussed briefly in section C. A description and the method for calculating the vibrational autocorrelation function are presented in section D. Spectroscopic results and the dynamic interpretations based on the vibrational autocorrelation function are discussed for each of the electronic states of $\mathrm{H}_{2} \mathrm{CCO}+$ and $\mathrm{D}_{2} \mathrm{CCO}^{+}$in section $\mathrm{E}$. The major conclusions are summarized in Section $\mathrm{F}$. 


\section{B . Experiment}

The molecular beam photoelectron spectrometer used for this study has been described in great detail by Pollard et al. ${ }^{18,19}$ and by Reutt et al. 20 Briefly, it consists of a differentially pumped supersonic molecular beam source for the introduction of the sample into the main chamber where ionization takes place; a differentially pumped windowless helium discharge lamp optimized to produce only the helium I $\alpha$ resonance line with minor contamination from higher resonances lines; a differentially pumped quadrupole mass spectrometer (QMS) to characterize the beam composition; a differentially pumped high resolution electron energy analyzer consisting of a $90^{\circ}$ spherical sector prefilter, a $180^{\circ}$ hemispherical analyzer equipped with a multichannel detector, and associated electron optics; and a dedicated microcomputer (LSI-11/73) for data acquisition and control. The electrons are collected at a $90^{\circ}$ angle with respect to the incident photon beam and the supersonic molecular beam and the intensity is uncorrected for angular distribution effects, since there are no ketene $B$ values for helium I $\alpha$ photons available.

Approximately 300 torr of ultra high purity helium ( $99.9999 \%$, Matheson) was first passed through a $\mathrm{U}$ tube maintained at $\mathrm{LN}_{2}$ temperature to remove trace amounts of water and other impurities in the carrier gas line, then bubbled through liquid monomer $\mathrm{H}_{2} \mathrm{CCO}$ or $\mathrm{D}_{2} \mathrm{CCO}$ maintained at Hexane/ $/ \mathrm{LN}_{2}$ slush temperature $\left(-96^{\circ} \mathrm{C}\right)$ with vapor pressures about 50 torr and 45 Torr as measured with an MKS model 122AA-2000 Baratron. The monomer $\mathrm{H}_{2} \mathrm{CCO}$ or $\mathrm{D}_{2} \mathrm{CCO}$ was prepared following a literature procedure 21-23 by thermodecomposition of acetic anhydride and $d_{6}$-acetic anhydride(Aldrich, $99.5 \% \mathrm{D}$-atom) at approximately $550^{\circ} \mathrm{C}$, trapping the acetic acid byproduct at $-78^{\circ} \mathrm{C}$ using dry ice/acetone slush and trapping ketene at $\mathrm{LN}_{2}\left(-195.8^{\circ} \mathrm{C}\right)$ temperature. Ketene prepared in this way was vacuum distillated once from a hexane $/ \mathrm{LN}_{2}$ slush temperature to $\mathrm{LN}_{2}$ temperature to remove trace amounts of acetic acid left and carbon dioxide, the

primary byproduct from ketene decomposition. Purified ketene and $D_{2}$-ketene were kept in 
the dark at $\mathrm{LN}_{2}$ temperature under continuous pumping until the photoelectron measurements started.

The $\mathrm{He} / \mathrm{H}_{2} \mathrm{CCO}$ or $\mathrm{He} / \mathrm{D}_{2} \mathrm{CCO}$ mixture with a total pressure of approximately 400 Torr was expanded through a $70 \mu \mathrm{m}$ diameter converging molybdenum nozzle held at room temperature, skimmed by a $0.858 \mathrm{~mm}$ diameter, $6.4 \mathrm{~mm}$ tall conical stainless steel skimmer, $D_{n s}=6.4 \mathrm{~mm}$. The beam source chamber pressure was $6 \times 10^{-5}$ torr, and the main chamber pressure was $3 \times 10^{-6}$ torr during the experimental measurements. All gas inlet lines were minimized and extensively baked $\left(200^{\circ} \mathrm{C}\right)$ under vacuum $\left(10^{-7}\right.$ torr) to remove trace amounts of water. Beam compositions were checked by the quadrupole mass spectrometer. No polymers of $\mathrm{H}_{2} \mathrm{CCO}$ or $\mathrm{D}_{2} \mathrm{CCO}$ or $\mathrm{CO}_{2}$ decomposition byproduct were found in the beam under these experimental conditions. The rotational temperature in the beams was estimated to be $\approx 5$ Kelvin as shown by LIF measurements under similar conditions. $24-26$

The complete photoelectron spectrum of each isotopic species was obtained as five sequential scans of the electron kinetic energy. Each scan was preceded and immediately followed by an argon calibration scan. To enhance statistics, four complete sets of spectra of $\mathrm{H}_{2} \mathrm{CCO}$ were scanned and summed. Thus the reported photoelectron bands each represent the summation of four such scans. Individual scans were made within a time period of less than two hours. The presented $\mathrm{D}_{2} \mathrm{CCO}$ photoelectron spectra were the summation of four such sets of scans as well. Restricting the length of each scan limits the total drift in the electron kinetic energy scale to $\leq 1 \mathrm{meV}$. The linearity of the kinetic energy scale was determined by obtaining the $\mathrm{N}_{2}^{+}$photoelectron spectrum and comparing the $\mathrm{N}_{2}^{+} \tilde{\mathrm{X}}$ $2 \Sigma_{g}^{+} \mathrm{V}=0$ and $\widetilde{\mathrm{B}}^{2} \Sigma_{\mathrm{u}}^{+} \mathrm{V}=0$ splitting with the accurate literature value of $3.16981 \mathrm{eV}$ available from $\mathrm{N}_{2}^{+}$optical emission spectroscopy. ${ }^{27}$ At higher kinetic energies, the linearity of the energy scale was checked by the photoelectron spectra of argon and xenon. The ionization potentials of these rare gases (I.P. $\mathrm{Ar}^{2} \mathrm{P}_{3 / 2}=15.75975 \mathrm{eV}$, and $\mathrm{Xe}^{2} \mathrm{P}_{3 / 2}=12.13000 \mathrm{eV}$ ) 
have been precisely determined from optical spectroscopy. ${ }^{28}$ The linearity of the molecular beam spectrometer is within $\pm 1.0 \mathrm{meV}$ over this entire energy range. The combination of the drift and the linearity of the energy scale errors limit the accuracy at which the absolute ionization potentials may be reported to $\pm 3.0 \mathrm{meV}$. Other spectroscopic constants, however, are obtained as line splittings and may be reported to a much higher accuracy of $\pm 0.5 \mathrm{meV}$.

\section{Vibronic Coupling and Isotope Effects on Vibronic Coupling}

In photoelectron spectroscopy, the vibronic coupling effect, that is the interaction of the electronic and nuclear (vibrational) motion, is often invoked to explain the observations that there are vibrational progressions in modes that are not allowed by the selection rules based on the Born-Oppenheimer approximation; ${ }^{29}$ that the observed vibrational frequencies are very low; and that odd quanta of non totally symmetric vibrations get excited. There are other couplings and resonances effects that can also cause similar effects, like vibrational-rotation coupling, rotation-electronic couplings and Fermi resonances. In general, these effects are much smaller than the vibronic coupling effect and are much easier to identify.

The vibronic coupling effects are usually treated as perturbations to the BornOppenheimer approximation; that is, the adiabatic Hamiltonian $\mathscr{H}_{0} .30$ We can expand the molecular Hamiltonian $\mathscr{K}(\mathrm{q} ; \mathrm{Q})$, which is a function of the electronic $\mathrm{q}$ and the nuclear (normal) $Q$ coordinates, in a Taylor series in the normal coordinates of vibration $Q$ near the equilibrium configuration $\mathrm{Q}_{0}$ :

$$
\mathscr{H}=\mathscr{H}(\mathrm{q} ; \mathrm{Q})_{\mathrm{Q}_{0}}+\sum_{\mathrm{r}=1}^{\infty}\left|\frac{1}{\mathrm{r} !} \frac{\partial^{\mathrm{r}} \mathscr{H}(\mathrm{q} ; \mathrm{Q})}{\partial \mathrm{Q}^{\mathrm{r}}}\right|_{\mathrm{Q}_{0}} \mathrm{Q}^{\mathrm{r}}+\cdots
$$


The first term in equation (3.1), $\mathscr{H}(\mathrm{q} ; \mathrm{Q}) \mathrm{Q}_{0}$, is called the zero-order Hamiltonian, that is the Born-Oppenheimer adiabatic Hamiltonian, and is usually expressed as $\mathscr{K}_{0}$. Applying the total Hamiltonian $\mathscr{X}$ on the molecular wave function $\Psi$ yields:

$$
\langle\Psi|\mathscr{E}| \Psi\rangle=\mathrm{E}_{0}+\mathrm{E}_{1}+\mathrm{E}_{2}+\ldots
$$

Where $E_{0}$ is the non-perturbed energy, and $E_{1}, E_{2}$ are the perturbation energies resulting from the linear and quadratic expansion terms in equation (4.1) respectively. The coupling of two wave functions $\Psi_{\mathrm{k}}$ and $\Psi_{\mathrm{j}}$ can be expressed according to the matrix elements:

$$
\left\langle\Psi_{\mathrm{k}}|\mathscr{H}| \Psi_{\mathrm{j}}\right\rangle
$$

which can be expanded with the Hamiltonian $\mathscr{X}$ of equation (4.1),

$$
\begin{aligned}
& \left\langle\Psi_{\mathrm{k}}|\mathscr{X}| \Psi_{\mathrm{j}}\right\rangle=\left\langle\Psi_{\mathrm{k}}\left|\mathscr{H}_{0}\right| \Psi_{\mathrm{j}}\right\rangle+\sum_{\mathrm{r}} \mathrm{Q}_{\mathrm{r}}\left\langle\Psi_{\mathrm{k}}\left|\frac{\partial \mathscr{X}}{\partial \mathrm{Q}_{\mathrm{r}}}\right| \Psi_{\mathrm{j}}\right\rangle+ \\
& +\frac{1}{2 !} \sum_{\mathrm{r}} \mathrm{Q}_{\mathrm{r}}^{2}\left\langle\Psi_{\mathrm{k}}\left|\frac{\partial^{2} \mathscr{X}}{\partial^{2} \mathrm{Q}_{\mathrm{r}}^{2}}\right| \Psi_{\mathrm{j}}\right\rangle+\frac{1}{2 !} \sum_{\mathrm{rS}} \mathrm{Q}_{\mathrm{r}} \mathrm{Q}_{\mathrm{s}}\left\langle\Psi_{\mathrm{k}}\left|\frac{\partial^{2} \mathscr{X}}{\partial \mathrm{Q}_{\mathrm{r}} \partial \mathrm{Q}_{s}}\right| \Psi_{\mathrm{j}}\right\rangle+\ldots
\end{aligned}
$$

The first term in equation (4.4) is always zero by the orthogonality relationship. Thus the necessary condition for the vibronic coupling effects to perturb the electronic states involved is that at least one term in equation (4.4) has a nonzero value that is not too small compared with the zero-order electronic states' energy separations.

The total Hamiltonian $\mathscr{H}$ of equation (4.1) always possesses the full symmetry of the molecular point group and transforms as $\Gamma_{1}$. This is also true for $\mathscr{X}_{0}$, the Hamiltonian for the zeroth-order unperturbed molecule. For the coupling operators in equation (4.4): 
$\partial \mathscr{W} / \partial Q_{r}$ transforms as the irreducible representation of the normal coordinate $Q_{r}, \Gamma\left(Q_{r}\right)$; $\partial^{2} \mathscr{2} / \partial^{2} Q_{r}$ always has at least one component that transforms as $\Gamma_{1}$, the full symmetry of the molecular point group; and $\partial 2 \mathscr{K} / \partial \mathrm{Q}_{\mathrm{r}} \partial \mathrm{Q}_{\mathrm{s}}$ transforms as $\Gamma\left(\mathrm{Q}_{\mathrm{r}}\right) \otimes \Gamma\left(\mathrm{Q}_{\mathrm{s}}\right)$. , The linear coupling integral in equation (4.4) will be finite if the product of the representations of all species in the integral contains the totally symmetric representation, $\Gamma_{1}$, that is:

$$
\Gamma\left(\Psi_{k}\right) \otimes \Gamma\left(\Psi_{j}\right) \otimes \Gamma\left(\partial \mathcal{K} / \partial Q_{r}\right) \subset \Gamma_{1}
$$

This will enable the $Q_{r}$ normal vibrational mode to couple and mix the $\Psi_{k}$ and $\Psi_{j}$ electronic states. If $\Psi_{k}$ and $\Psi_{j}$ are degenerated states, then $Q_{r}$ is the asymmetric vibrational mode that will remove the degeneracy. We can discuss the two quadratic terms in equation (4.4) similarly. The linear terms are usually much larger than the quadratic terms, and the quadratic terms are normally neglected when the linear terms are nonzero. In a polyatomic molecule, there could be more than one normal mode $Q_{r}$ that satisfies the condition of equation (4.5). Those normal modes that satisfy equation (4.5) are called vibronically active modes. When there are more than one mode that are vibronically active, multimode vibronic coupling effects will occur. In general, these are more complicated to deal with. Multimode vibronic effects and their influence on spectroscopy have been reviewed in detail by Köppel et al. 31

It can be seen from equation (4.4) that both the linear and quadratic vibronic couplings depend on the normal coordinates that are mass-dependent. It is expected that the couplings will be different for isotope-substitued compounds in the same electronic state. One important consequence of this effect is that it is possible for different vibration modes to be excited to a different extent for isotope-substituted compounds. This will cast new difficulties in the interpretation of photoelectron spectra of isotopic compounds. 
Theoretical calculations have shown that this effect appears in isotope-substituted compounds. ${ }^{32,33}$ The $\mathrm{H}_{2} \mathrm{CCO}^{+}$and $\mathrm{D}_{2} \mathrm{CCO}^{+}$photoelectron spectra have shown this kind of behavior, and they will be discussed further in section $\mathrm{E}$ of this chapter.

\section{The Correlation Function}

The autocorrelation function formalism description of the intramolecular dynamic process gives complementary information to time-dependent measurements. Lorquet et al.. 11-14 first demonstrated how this can be done for photoelectron bands using the formalism of Heller. 7-10 A variation on the method of Lorquet et al. was used by Pollard et al., 15 and by Reutt et al. 16,17 to study the dynamic characteristics of intramolecular processes using supersonic molecular beam photoelectron spectroscopy. Ruscic 28b has also given the analytical form of the vibrational autocorrelation function for photoelectron bands under the harmonic approximation for vibrational motions.

Very recently, Remacle et al. ${ }^{14}$ have suggested that there exists a relationship between the vibrational autocorrelation function $\mathrm{C}(\mathrm{t})$ and the population decay function $\mathbf{P}(\mathrm{t})$ of a particular electronic state. They defined an average population decay curve $\mathbf{P}_{\mathrm{av}}(t)$ valid in a particular time period and $C_{a v}(t)$, the average correlation function, obtained from $C(t)$. It was pointed out by the authors that for a specific excitation, the exact initial rate of decay of $\mathbf{P}_{\mathrm{av}}(t)$ (valid up to the dephasing time $T_{1}$ ) is equal to the initial rate of decay of $|C(t)|^{2}$, and the subsequent time evolution of $P_{a v}(t)$ can be obtained from $\left|C_{a v}(t)\right|^{2}$ values that are derived by averaging $|C(t)|^{2}$ over its oscillation. To a good approximation, the average population decay curve $P_{a v}(t)$ can be obtained by multiplying $\left|C_{a v}(t)\right|^{2}$ with an appropriate constant: i.e., the slopes of the two curves are the same.

The autocorrelation function can be expressed as

$$
C(t)=|\langle\phi(0) \mid \phi(t)\rangle|
$$


Here $\phi(0)$ is the initial nuclear wavefunction, and $\phi(t)$ is the time evolution of this wavefunction on the excited state potential-energy surface. $C(t)$ represents the probability amplitude that at time $t$ the system remains in the initially prepared state. It must be noted that the correlation function is the measure of the time evolution of the initial wave packet on the excited-state potential energy surface and not a measure of the excited state population. But, as mentioned in the preceding paragraph, the initial decay rate of $C(t)$, through $|C(t)|^{2}$, is the same as the average population $\mathbf{P}_{\mathrm{av}}(t)$ initial decay rate. It is dominated by the dephasing of the initial wave packet due to the different shape of the excited-state potential energy surface. Only at times greater than a vibrational period will radiationless decay processes appear in the correlation function time evolution. The derivation of the autocorrelation function $\mathbf{C}(\mathrm{t})$ from photoelectron spectroscopy experimental data has been reported; 12 only the principal points will be outlined here. The form of photoionization cross section under the strict Franck-Condon approximation 34 can be expressed as

$$
\left.\sigma(\mathrm{E}) \propto|| \mathrm{M}_{\mathrm{el}}(\mathrm{Q}, \mathrm{E})|2|\left\langle\Psi^{\prime \prime} \mid \Psi^{\prime}\right\rangle\right|^{2} \mid
$$

Here $\mathbf{M}_{\mathrm{el}}(\mathrm{Q}, \mathrm{E})$ is the pure electronic transition moment that is a function of the nuclear coordinates $Q$ and electron kinetic energy $E$, and $\Psi^{\prime \prime}$ and $\Psi^{\prime}$ are the initial- and final-state vibrational wave functions. The electronic transition moment varies slowly over the photoelectron band. In lieu of any arbitrary approximation, a constant value was used in calculating the correlation functions reported here.

Applying the completeness conditions of a set of eigenstates $\Psi$ " of the molecular Hamiltonian and invoking the analytical form of the Dirac $\delta$ function, it has been shown 12 that the cross section for photoionization becomes 


$$
\sigma(E) \propto \frac{1}{2 \pi} \int_{-\infty}^{+\infty} e^{\mathrm{i} E \mathrm{t} / \hbar}\left\langle\Psi^{\prime \prime}\left|\mathrm{e}^{-\mathrm{i} \Psi t / \hbar}\right| \Psi^{\prime \prime}\right\rangle d t
$$

Here $\mathscr{X}$ is the molecular Hamiltonian, $\Psi^{\prime \prime}$ is identified as the initial nuclear wave function $\phi(0)$, and $\mathrm{e}^{-\mathrm{i} X t / \hbar}\left|\Psi^{\prime \prime}\right\rangle$ is the nuclear wave function at time $\mathrm{t}: \phi(\mathrm{t})$. This gives

$$
\sigma(E) \propto \frac{1}{2 \pi} \int_{-\infty}^{+\infty} e^{i E t / \hbar}\langle\phi(0) \mid \phi(t)\rangle d t
$$

and the correlation function can be obtained by a Fourier transformation of the cross section

$$
C(t)=|\langle\phi(0) \mid \phi(t)\rangle| \propto \frac{1}{2 \pi} \int_{-\infty}^{+\infty} \sigma(E) e^{-i E t / \hbar} d t
$$

It can be seen from this equation that the correlation function can be obtained from the Fourier transform of the photoelectron partial cross section $\sigma(E)$. This can be accomplished by deconvoluting the instrument response function $\mathbf{I}(E)_{\text {ir }}$, which we determine from the photoelectron spectrum of a rare gas at a kinetic energy comparable to the band of interest, from the quantity $I(E)$, intensity vs. energy, which we measure in photoelectron spectroscopy. The contributions of the finite rotational temperature in a supersonic molecular beam experiment (typically $\sim 5-10 \mathrm{Kelvin}$ ) and the rotational excitations contribution to the correlation function were removed by convoluting $I(E)_{\text {ir }}$ with a gaussian function $\mathrm{I}(\mathrm{E})_{\mathrm{g}}$ to generate a rotationally broadened instrument response function. The width of the gaussian function was chosen from the average rotational 
constant , $\mathbf{B}_{\mathrm{avg}}$, of the molecular ion for the particular electronic state (if available), the finite rotational temperature, and the rotational selection rules for photoionization. Ruscic 13 has taken a different approach to account for all the corrections. However, both methods give essentially the same results in the time window of interest.

The procedure for calculating the correlation function of $\widetilde{\mathrm{X}}, \widetilde{\mathrm{A}}, \widetilde{\mathrm{B}}, \widetilde{\mathrm{C}}, \widetilde{\mathrm{D}}$, and $\widetilde{\mathrm{E}}$ states of ketene and $d_{2}$-ketene is the following. First, the band of interest was isolated (in the case of $\tilde{\mathrm{A}}$ and $\tilde{\mathrm{B}}$ states, they were digitally separated to remove the overlapping of these two bands. The heavy overlapping of the $\tilde{C}$ and $\tilde{D}$ states, however, renders such separation impractical, and was not attempted ), and the empirically determined background, plus any constant background was then removed. The resulting band was then normalized (area $=1$ ) and Fourier transformed using a discrete FFT algorithm. ${ }^{35}$ The instrument response function was similarly normalized and convoluted with a Gaussian of $3 \mathrm{meV}$ (FWHM). The resulting function was Fourier transformed and divided into that of the data. Finally, the modulus of the previous result was calculated which gave the correlation function. The procedure can be summarized by the following equation:

$$
\begin{aligned}
& C^{v i b}(t)=|\langle\phi(0) \mid \phi(t)\rangle|=\left|\int_{-\infty}^{+\infty} I(E) e^{-i E t / \hbar} d E\right| \div \\
& \left|\int_{-\infty}^{+\infty} I(E)_{i r} e^{-i E t / \hbar} d E X \int_{-\infty}^{+\infty} I(E)_{g} e^{-i E t / \hbar} d E\right|
\end{aligned}
$$

Then $|C(t)|^{2}$ was then calculated, and $\left|C_{a v}(t)\right|^{2}$ was evaluated by fitting $|C(t)|^{2}$ with an exponential curve of the form $\mathrm{Ae}^{-\mathrm{ikt}}$ for both the initial drop and the subsequent decay after the first 'vibrational' period. 


\section{E. Results and Discussions}

The full photoelectron spectra of $\mathrm{H}_{2} \mathrm{CCO}^{+}$and $\mathrm{D}_{2} \mathrm{CCO}^{+}$obtained by combining four separate scans with a resolution of $11.5 \mathrm{meV}$ FWHM (as measured with $\operatorname{Ar}{ }^{2} \mathrm{P}_{1 / 2}$, ${ }^{2} P_{3 / 2}$ ) are shown in Figure 1 and Figure 2, respectively. Table I summarizes the measured spectroscopic constants together with results reported in the literature. ${ }^{38-40}$

\section{First Band, $\tilde{\mathrm{X}} \mathbf{1}^{2} \mathrm{~B}_{1}$ State}

The ground states of both $\mathrm{H}_{2} \mathrm{CCO}^{+}$and $\mathrm{D}_{2} \mathrm{CCO}^{+}$showed well-resolved vibrational fine structures, as presented separately in Figure 3 and Figure 4. Observed spectroscopic results are presented in Table II. In the present work, effective rotational cooling of the sample by supersonic expansion permits all of the observed vibrational levels to be determined with much improved accuracy, and the mean energies of these transitions were determined by the following least-squares fitting procedure. The adiabatic transitions (or the most intense feature) were first determined by fitting these features to Gaussians. The adiabatic peaks were then isolated and used as empirical functions to fit the successive levels. This procedure allows the values of the peak splittings to be determined to an accuracy of $\leq \pm 0.0005 \mathrm{eV}$. The mean transition energies located through this procedure are listed for each of the bands observed. The vibrational levels for each normal mode were then least-squares fitted to the standard energy level expression of a Morse oscillator 36,37

$$
\mathbf{G}_{i}^{0}(v)=\omega_{i}^{0}(v)-\omega_{i}^{0} x_{i}^{0}(v)^{2}
$$

with the zero point energy being set to zero, $\omega_{i}^{0}$ and $\omega_{i}^{0} x_{i}^{0}$ are related to $\omega_{i}$ and $\omega_{i} x_{i}$ in the following ways: $\omega_{i}=\omega_{i}^{0}+\omega_{i}^{0} x_{i}^{0} ; \omega_{i} x_{i}=\omega_{i}^{0} x_{i}^{0}$. Here $\omega_{i}$ is the fundamental vibratinn frequency, and $\omega_{\mathrm{i}} x_{\mathrm{i}}$ is the quadratic anharmonicity constant for the ith normal vibration mode. 
The result shows that at least two vibrational modes are strongly excited in this state of $\mathrm{H}_{2} \mathrm{CCO}^{+}$, and for $\mathrm{D}_{2} \mathrm{CCO}^{+}$there are additional fine structures in one of the vibrational progressions which is not apparent from the $\mathrm{H}_{2} \mathrm{CCO}^{+}$spectrum, indicating that there are more than two modes that are excited strongly. The abnormality in the intensity of the third peak of the $\tilde{\mathrm{X}} 1^{2} \mathrm{~B}_{1}$ state of $\mathrm{H}_{2} \mathrm{CCO}^{+}$and $\mathrm{D}_{2} \mathrm{CCO}^{+}$was not explained in the original Baker paper and was not discussed in the study by Hall et al. either. Takeshita attributed this intensity abnormality to the superposition of the two possible vibrational levels, $v_{2}=1$ and $V_{4}=2$. The present study supports the theoretical calculation by noticing that $v_{2} \approx 2 v_{4}$, but the two peaks are not exactly superimposed. Instead, the abnormality of the intenstity of this peak was attributed to a Fermi resonances, in which one peak "borrows" intensity from its nearby neighbor. The weak excitation of the $\mathrm{v}_{3}$ mode in $\mathrm{H}_{2} \mathrm{CCO}^{+}$was further established by the $\mathrm{D}_{2} \mathrm{CCO}^{+}$spectrum, where a reduction in frequency was apparent for the excitation of the $v_{3}$ mode which accounts for the doublet-like structure in the second, the fourth, and the sixth peaks in the $\mathrm{D}_{2} \mathrm{CCO}^{+}$spectrum. This observation also indicates that the vibronic couplings of the two isotopic molecules in this state are different. This accounts for the strong excitation of the $\mathrm{V}_{3}$ mode in $\mathrm{D}_{2} \mathrm{CCO}{ }^{+}$compared with that of $\mathrm{H}_{2} \mathrm{CCO}^{+}$. In other words, the potential energy surfaces of the $\widetilde{\mathrm{X}} 1^{2} \mathrm{~B}_{1}$ state for $\mathrm{H}_{2} \mathrm{CCO}^{+}$ and $\mathrm{D}_{2} \mathrm{CCO}^{+}$are different, especially along the $\mathrm{Q}_{3}$ normal coordinate in the FranckCondon region. The strong excitation of $v_{2}$ and $v_{3}, v_{4}$ modes in the $\tilde{X} 1^{2} B_{1}$ state of $\mathrm{H}_{2} \mathrm{CCO}^{+}$and $\mathrm{D}_{2} \mathrm{CCO}^{+}$and the intensity of the adiabatic peak indicate that the electron being ejected is mostly from the non-bonding oxygen lone pair.

The vibrational autocorrelation functions calculated from the $\widetilde{\mathrm{X}} 1^{2} \mathrm{~B}_{1}$ state of each ion are shown in Figure 5 and Figure 6. Very similar oscillatory patterns are evident for each isotopic species. The beat pattern results from the phase relationship of a stable twomode anharmonic oscillator system with $v_{2} \geq 2 v_{4}, 2 v_{3}$. At shorter times a dephasing of the wave packet, due to the differences in the ionic and neutral potential energy surfaces, 
dominates the correlation functions. After 10.4 fs for $\mathrm{H}_{2} \mathrm{CCO}^{+}\left(10.5\right.$ fs for $\left.\mathrm{D}_{2} \mathrm{CCO}^{+}\right)$the fastest components of the wave packet return to the initial positions on the ionic potential energy surfaces and a reduced maximum of the correlation functions of 0.38 ( 0.42 for $\mathrm{D}_{2} \mathrm{CCO}^{+}$) is achieved. This corresponds very closely to one period of $\mathrm{v}_{2}$ vibration motion. The slower components of the wave packet, which must also travel along the $v_{3}, v_{4}$ coordinates, are expected to return to the initial position on the ionic potential energy surfaces after approximately one period of $v_{4} / N_{3}$ vibration motion. The observed maximums in the correlation functions of 0.78 at $30.5 \mathrm{fs}$ and 0.83 at $30.8 \mathrm{fs}$ for $\mathrm{H}_{2} \mathrm{CCO}^{+}$ and $\mathrm{D}_{2} \mathrm{CCO}^{+}$, respectively, are mainly attributed to this return. The small shifts in times of the observed peaks in the correlation functions arise from the spreading of the wave nacket on the anharmunic potential energy surfaces and the relative phase relationships between the $v_{2}$ and $v_{3}, v_{4}$ normal modes.

High correlation is maintained at longer times ( $>0.3$ at $200 \mathrm{fs}$ ), which indicates that the initially prepared wave packet is fairly stable regarding deformations along the $Q_{2}$ and $\mathrm{Q}_{3} / \mathrm{Q}_{4}$ normal coordinates. Relatively shallow minimums observed in the correlation function are the characteristics of the wave packet prepared through a predominately adiabatic transition. The initially prepared wave packet is mostly localized around the minimum of the upper potential energy surfaces along the $Q_{2}$ and $Q_{3} / Q_{4}$ normal coordinates, and weakly oscillates around this region, retaining substantially high level correlation at all times.

The isotope effect is also noticeable in the correlation functions, referring to Figure 7 , where the correlation functions of the $\widetilde{\mathrm{X}} 1^{2} \mathrm{~B}_{1}$ state of $\mathrm{H}_{2} \mathrm{CCO}+$ and $\mathrm{D}_{2} \mathrm{CCO}^{+}$are plotted together. At shorter times it can be seen that the over all shapes and trends in the correlation function for both isotopic species are almost identical, indicating that the predominate characters of the normal vibration modes involved have very little contribution 
from the $\mathrm{CH}_{2}$ or $\mathrm{CD}_{2}$ groups. At lungc: imes, however, the differences between the correlation functions of the isotopic species are apparent. This can be attributed largely to the differences in the ionic potential energy surfaces between the isotopic species as incicated by the different anharmonicity constants for each normal mode.

\section{The Second Band, $\tilde{A} \mathbf{1}^{2} B_{2}$ State}

The second band of ketene for both isotopic species, which showed well-resolved vibrational fine structures albeit overlapped strongly with the third band $\widetilde{\mathrm{B}} 2^{2} \mathrm{~B}_{1}$ state, are presented in Figure 8 and Figure 9, which show the $\widetilde{\mathrm{B}} 2^{2} \mathrm{~B}_{1}$ state of both isotopic species as well. Observed spectroscopic constants for these states are summarized in Table III. In the present work, effective rotational cooling and the very high resolution achieved unveiled the details of the vibrational progressions in the $\tilde{A} 1^{2} \mathrm{~B}_{2}$ state for both isotopic species for the first time. The vibrational assignments for the $\tilde{A} 1^{2} B_{2}$ state are well established in the present study based on the $a b$ initio calculation by Takeshita, except for the possible excitation of one other 'soft' mode which causes the doublet-like structures observed in the first few vibrational progressions of this band for both isotopic species. Hall et al. ${ }^{4}$ explained the complexity of this band in terms of predissociation of the cation in this state, quoting no detectable emission from this state as an indication for the short life-time implied. Takeshita, however, in an $a b$ initio calculation, showed that the cation in this state is stable, and the $C_{2} v$ geometry is a true equilibrium configuration. He attributed the complexity of this band to the possible contamination from the $\mathrm{CO}_{2}^{+} \widetilde{\mathrm{X}}{ }^{2} \Pi_{\mathrm{g}}$ band near $13.8 \mathrm{eV}$ and from the $\widetilde{\mathrm{B}} 2^{2} \mathrm{~B}_{1}$ state of ketene above $14.6 \mathrm{eV}$.

We can first eliminate the possibility of the $\mathrm{CO}_{2}^{+} \widetilde{\mathrm{X}}^{2} \Pi_{\mathrm{g}}$ band contamination from the present spectrum of this band. The $\mathrm{CO}_{2}^{+} \tilde{\mathrm{X}}^{2} \Pi_{\mathrm{g}}$ band AIE is $13.7778 \mathrm{eV}{ }^{41}$, and the $20 \mathrm{meV}$ spin-orbital splitting present in the band would have been evident if there were any contributions from the $\mathrm{CO}_{2}^{+} \tilde{\mathrm{X}}^{2} \Pi_{\mathrm{g}}$ band. Also, experiments performed at hexane $\mathrm{LN}_{2}$ 
slush $\left(-96^{\circ} \mathrm{C}\right)$ temperature $\left(\mathrm{CO}_{2}\right.$ vapour pressure $\leq 100$ torr $)$ and at acetone/dry ice slush ($\left.78^{\circ} \mathrm{C}\right)$ temperature $\left(\mathrm{CO}_{2}\right.$ vapour pressure $\geq 760$ torr $)$ showed no sign of changes in relative intensities for this band. This and the QMS diagnosis results establish that the ketene sample used for this study had not been contaminated by the dissociation of ketene byproduct $\mathrm{CO}_{2}$. The contributions from the $\widetilde{\mathrm{B}}{ }^{2} \mathrm{~B}_{1}$ band of the ketene cation can be rejected, because the very distinct long vibrational progressions observed for the $\widetilde{\mathrm{B}} 2^{2} \mathrm{~B}_{1}$ band in both isotopic species cannot possibly broaden nor complicate the vibrational progressions of the $\tilde{\mathrm{A}} 1^{2} \mathrm{~B}_{2}$ state. We attribute the complexity of this band to the possible predissociation of this band by another close-lying repulsive state or the possibility of vibrational predissociation of this band above $14.5 \mathrm{eV}$. The tail of this band extends all the way to the next observed band, the $\widetilde{B} 2^{2} B_{1}$ state. The relatively short vibrational progressions observed in this $\left(\tilde{\mathrm{A}} 1^{2} \mathrm{~B}_{2}\right)$ band for both isotopic species supports the predissociation mechanism. The major vibrational progression observed is attributed to the excitations of the $V_{2}$ and $V_{4}$ modes. The apparent insensitivity to isotope substitution of the vibrational progressions implies that the $V_{2}$ and $V_{4}$ modes are predominately $C=C=O$ stretching motions. The differences in the observed vibrational frequencies excluded the validity of the relationships: $v_{2} \approx 2 v_{4}$ and $v_{2} \approx V_{3}+V_{4}$ shown in the calculated vibrational frequen:ies by Takeshita. The doublet-like structures observed in the first few vibrational progressions indicate that at least one 'soft' mode has been excited along with the excitation of the $v_{2}$ and $V_{4}$ modes. The large reduction in the splittings among the doublet-like structure upon deuteration $\left(\sim 460.54 \mathrm{~cm}^{-1}\right.$ in the $\tilde{\mathrm{A}} 1^{2} \mathrm{~B}_{2}$ state of $\mathrm{H}_{2} \mathrm{CCO}^{+}$vs. $\sim 299.32 \mathrm{~cm}^{-1}$ of $\mathrm{D}_{2} \mathrm{CCO}^{+}$, calculated from the first doublet structure in this band for both isotopic species) implied that this mode might have involved the $\mathrm{CH}_{2}$ and $\mathrm{CD}_{2}$ group in the molecular ions. Following the convention of Duncan et al. ${ }^{38,39}$, Allen and Schaefer ${ }^{42-44}$ of orienting the molecule on the yz plane, this is most likely the $v_{5}$ mode, which is characterized by the $\mathrm{CH}_{2}$ group out of plane wagging motion, that is excited. Without supporting evidence from other 
measurements, especially rotationally resolved spectroscopic studies on the cation in this state, the assignment of the $v_{5}$ mode could only be regarded as very tentative at best. Further theoretical characterization of the potential energy surface of this state would be extremely helpful in this regard. The strong couplings among vibrational modes revealed by the high-resolution optical studies of Duncan et al. on the neutral $\tilde{X}^{1} A_{1}$ ground state of ketene showed the complexity of the vibrational modes in ketene. The same or even more complex couplings among vibrational modes can be expected for the ketene cations, as shown in this study for this band.

The correlation functions calculated for this state in both isotopic species, by digitally removing the $\widetilde{\mathrm{B}} 2^{2} \mathrm{~B}_{1}$ state, are shown in Figure 10 and 11 . The most striking feature of the correlation functions is the loss of correlation strengtiz on a very short time scale. After only $6.2 \mathrm{fs}$ in $\mathrm{H}_{2} \mathrm{CCO}^{+}$and only $7.5 \mathrm{fs}$ in $\mathrm{D}_{2} \mathrm{CCO}^{+}$the correlation functions reached only 0.15 and 0.01 , respectively. Thereafter, the correlation functions never regained their initial strengths, and there were no recognizable major oscillations. The ultra fast loss of correlation stength indicates that the initially prepared wave packet was displaced substantially from the minimum of the upper potential energy surface. The absence of the return of major oscillations strength implied that the wave packet never got back to its initial position on the upper potential energy surfaces and never regained its initial shape. Adopt the method of Remacle et al., ${ }^{14}$ the initial and subsequent drops of the correlation functions $\left([C(t)]^{2}\right)$ were fitted to the exponential form of $A \cdot e^{-k t}$. We got $k=0.95\left(\mathrm{fs}^{-1}\right)$ and 0.01 for $\mathrm{H}_{2} \mathrm{CCO}^{+}$, and $\mathrm{k}=0.70$ and 0.01 for $\mathrm{D}_{2} \mathrm{CCO}^{+}$. This led to the extremely fast population decay rate on the order of $1.1 \mathrm{fs}$ and $1.4 \mathrm{fs}$ for the initial, $100 \mathrm{fs}$ for the subsequent decaying for ketene cations. The 100 fs decaying could only be regarded as an order of magnitude, uince the correlation strengths were very weak in both isotopic species after the initial decay. As discussed by Remacle et al., the initial drops in correlation functions calculated from photcelectron spectra are very sensitive to the 'wings' 
in the photoelectron spectra. The experimental control of the wings was extremely difficult due to the low signal-to-noise ratio. Thus the calculated initial population-decay rate constants could only be used as indications of the order of magnitude involved. The extremely fast initial decay of the population, as manifested by the correlation function of this state on the order of a few femtosecond, implied upper potential energy surfaces very different from the neutral ground state ones according to the theoretical treatment of Ruscić. 13 The absence of any detectable emissions from this state implied that ultra-fast intramolecular processes are important in the decay of the cation from this state. This also supports the argument presented above that ketene cation in the $\tilde{\mathrm{A}} 1^{2} \mathrm{~B}_{2}$ state might have been predissociated in the Franck-Condon region.

\section{Third Band, $\widetilde{\mathrm{B}} \mathbf{2}^{2} \mathrm{~B}_{1}$ State}

The third bands of ketene for both isotopic species, with fully resolved vibrational fine structures, are presented in Figure 8 and 9. The observed spectroscopic constants are summarized in Table IV. The adiabatic ionization energy was clearly established by the present study to be $14.6089(8)$ and $14.6106(5) \mathrm{eV}$ for $\mathrm{H}_{2} \mathrm{CCO}$ and $\mathrm{D}_{2} \mathrm{CCO}$. Takeshita 6 has placed the AIE for this band around $14.38 \mathrm{eV}$, based largely on his FCF calculations. Regarding the $\mathrm{H}_{2} \mathrm{CCO}^{+}$spectrum of this band, however, if the AIE were lower than $14.609 \mathrm{eV}$, we would have seen another sharp peak in the spectrum near $14.50 \mathrm{eV}$ that would stand out compared with contributions from the $\tilde{\mathrm{A}} 1^{2} \mathrm{~B}_{2}$ state. The observed abnormality in intensity in the fifth peak of the $\mathrm{H}_{2} \mathrm{CCO}^{+}$spectrum might be attributed to a Fermi resonances. In addition to the main vibrational progression, which we assigned as the $V_{4}$ mode, there was another much weaker progression with similar spacing. The splitting between the two progressions observed is $346.7 \pm 8 \mathrm{~cm}^{-1}$ which, as in the $\tilde{\mathrm{A}} 1^{2} \mathrm{~B}_{2}$ state, might be assigned to another 'soft' mode $v_{6}$, that may be characterized as the 
$\mathrm{C}=\mathrm{C}=\mathrm{O}$ skeleton out-of-plane bending, based largely on the insensitivity of this splitting to deuteration as discussed below.

For the $\mathrm{D}_{2} \mathrm{CCO}^{+}$spectrum, the present high-resolution study revealed more fine structure than observed previously by both Baker et al. and Hall et al.. The additional vibrational progressions present in $\mathrm{D}_{2} \mathrm{CCO}^{+}$beyond those present in the $\mathrm{H}_{2} \mathrm{CCO}^{+}$spectrum seerned to be the combination of exciting the $v_{4}$ mode together with one quantum of the $v_{3}$ mode. This assignment was largely based on the ab initio calculation on this band by Takeshita, ${ }^{6}$ although the calculated spectrum appeared to be very simple and has much less fine structure due to the inadequacy inherent in the calculation. The excitation of four vibrational modes in this band makes the definitive assignment of this band extremely difficult especially regarding the assignment of the excitation of $\mathrm{V}_{6}$, just as in the $\mathrm{H}_{2} \mathrm{COO}^{+}$ spectrum of this band. Nonetheless, the present study can be expected to spur theoretical interests to perform high level, multimode calculations to further our understanding on the structure and spectroscopy of ketene cation in the $\widetilde{\mathrm{B}} 2^{2} \mathrm{~B}_{1}$ state in the near future. And again, the differences in vibrational excitations and length of the progressions observed in $\mathrm{D}_{2} \mathrm{CCO}^{+}$implied that the ionic potential energy surface in the Franck-Condon region and the vibronic couplings, are different for isotopic molecules, as discussed in section $\mathrm{C}$ of this chapter.

The correlation functions calculated for this band for both isotopic species, are shown in Figures 12 and 13. The fast initial drop in correlation strength and the deep valleys in the correlation function indicated a substantially different upper potential energy surface than the neutral ground state one for both isotopic species in the Franck-Condon region. The initial position of the wave packet was displaced from the equilibrium geometry, which is consistent with the observed extensive vibrational progressions. The wave packet oscillated with rather large amplitude on the upper potential energy surface, mostly along the $\mathrm{Q}_{4}$ normal coordinate. After 35.1 fs for $\mathrm{H}_{2} \mathrm{CCO}+\left(35.2\right.$ fs for $\left.\mathrm{D}_{2} \mathrm{CCO}^{+}\right)$ 
the wave packet returned to the initial position on the ionic potential energy surfaces and a reduced maximum of $C(t) 0.86(0.79)$ was achieved. This corresponded very closely to one period of $V_{4}$ vibrational motion, and the major oscillation in the correlation function for both isotopic species had a period close to the $v_{4}$ normal mode. The ionic potential energy surface thus is strongly bound along the $\mathrm{Q}_{4}$ normal coordinate. The slow decaying of the major oscillation of the correlation function was attributed to the spreading of the wave packet due to the anharmonicity of the potential energy surfaces and the weak excitations of other normal modes. The calculated $[C(t)]_{a v}^{2}$ indicated a population decay rate on the order of $100 \mathrm{fs}$ for both isotopic species. The multiplet-like structures in the photoelectron spectrum of $\mathrm{D}_{2} \mathrm{CCO}^{+}$of this band, however, induced additional spreading of the wave packet, which accounted for the loss of correlation to noise level for this band after $74 \mathrm{fs}$. The stability of this state also supported the assignment of the $\widetilde{\mathrm{A}} 1^{2} \mathrm{~B}_{2}$ state proposed in the preceding section: that the $\tilde{\mathrm{A}} 1^{2} \mathrm{~B}_{2}$ state might be predissociated. The gradually rising 'background' observed in the $\widetilde{B} 2^{2} B_{1}$ state of the ketene cation spectrum was caused mainly by the overlapping tail of the $\tilde{\mathrm{A}} 1^{2} \mathrm{~B}_{2}$ state.

\section{The Fourth and Fifth Bands, $\widetilde{C} 2^{2} B_{2}$ and $\tilde{D} 1^{2} A_{1}$ States}

The photoelectron spectra of these two bands, as show in Figure 14 and 15, overlapped heavily, and they will be discussed together here. Observed spectroscopic constants are tabulated in Table $V$ for the $\widetilde{C}{ }^{2} B_{2}$ state, and in Table VI for the $\widetilde{D} 1^{2} \mathrm{~A}_{1}$ states. The $\widetilde{\mathrm{C}} 2^{2} \mathrm{~B}_{2}$ states of both isotopic species had well characterized vibrational progressions and are very similar in appearance. The major vibrational progression, upon deuteration, had a significant reduction in frequency and was assigned to the $v_{3}$ mode, supporting the assignment of Hall et al.. ${ }^{4}$ Takeshita, ${ }^{6}$ however, favored the assignment of this progression to the $v_{4}$ mode. As discussed for the $\widetilde{\mathrm{X}} 1^{2} \mathrm{~B}_{1}$ state, the strong coupling and mixing among different vibrational modes in ketene made the assignment only tentative 
without rotationally resolved studies. The gradually rising 'background' towards higher IEs of the $\widetilde{C}{ }^{2}{ }^{2}{ }_{2}$ state were mostly attributed to the contributions by the overlapping, dissociative $\tilde{\mathrm{D}} 1^{2} \mathrm{~A}_{1}$ state. The AIE of the $\tilde{\mathrm{D}} 1^{2} \mathrm{~A}_{1}$ state listed in Table $\mathrm{V}$ thus can only be regarded as the best estimate along in the same spirit, because of the completely dissociative nature of this band in the Franck-Condon region. The ab initio calculation results, however, gave spacings among vibrational progressions on the order of $40 \mathrm{meV}$ for the $\tilde{\mathrm{D}}$ $1^{2} \mathrm{~A}_{1}$ state with strong excitation of the $V_{4}$ normal mode along with weak excitations of the $v_{1}$ and $v_{3}$ normal modes. Thus it was rather unexpected that the present high resolution study did not unveil these progressions fully. The qualitative disagreements between the calculated and the experimental spectra for the $\tilde{\mathrm{D}} 1^{2} \mathrm{~A}_{1}$ state thus indicated that the potential energy surfaces of the cation in the Franck-Condon region must be very different from the neutral ground state and have a large anharmonicity constant in disagreement with the theoretical calculated spectrum based on harmonic potentials. Further high level calculations, and possible rotationally resolved studies by other spectroscopic means, would be very helpful in solving this difficulty.

The heavy overlapping of these two states precluded any simple means of separating them. No attempt was made to calculate the correlation functions for these two states. However, the overall FWHM of $12.5 \mathrm{meV}$ for most of the peaks observed in the $\tilde{\mathrm{C}}$ ${ }^{2} \mathrm{~B}_{2}$ state and the shapes of the peaks indicated that the $\widetilde{\mathrm{C}}{ }^{2} \mathrm{~B}_{2}$ state was bound in the Fracnk-Condon region. The rather broad and complicated band shape of the $\tilde{\mathrm{D}} 1^{2} \mathrm{~A}_{1}$ state, on the other hand, indicated that the $\tilde{D} 1^{2} A_{1}$ state might be repulsive over part of the potential energy surface probed by photoelectron spectroscopy. The full nature of the potential energy surface for this state awaits further theoretical and experimental investigations. 


\section{The Sixth Band, $\widetilde{E} 2^{2} A_{1}$ State}

The photoelectron spectra of this band for both isotopic species are shown in Figures 16 and 17. The measured spectroscopic information is summarized in Table VII. Earlier experimental studies on this band had been impeded by the presence of $\mathrm{CO}_{2}$ impurity and by the possible overlapping from the $\mathrm{CO}_{2}^{+} \widetilde{\mathrm{B}} 2 \Sigma_{\mathrm{u}}^{+}$state. The present spectrum showed essentially no contamination from the $\mathrm{CO}_{2}^{+} \widetilde{\mathrm{B}}{ }^{2} \Sigma_{\mathrm{u}}^{+}$state as discussed above with the $\tilde{A} 1^{2} B_{2}$ state of ketene and $d_{2}$-ketene. Even with the present high resolution and the effective rotational cooling, the width of the adiabatic peak for both isotopic species was much broader than the instrumental resolution and cannot be accounted for by invoking rotational broadening. The width, almost $40 \mathrm{meV}$ FWHM for the adiabatic peaks, and the asymmetric shapes of the peaks, as well as the absence of any major vibrational progression, implied that other intramoleclular processes were at play.

The correlation function calculated for this state is shown in Figures 18 and 19 for $\mathrm{H}_{2} \mathrm{CCO}^{+}$and $\mathrm{D}_{2} \mathrm{CCO}^{+}$, respectively. The ultra fast loss of correlation strength and the absence of the any major oscillation in the correlation function indicates lifetime broadening. The population decay rates calculated are $\tau(1 / \mathrm{k})$ equal to: 3 and 4 fs for the initial drop; and 22.2 and 28.5 fs for the late decay for $\mathrm{H}_{2} \mathrm{CCO}^{+}$and $\mathrm{D}_{2} \mathrm{CCO}^{+}$, respectively. These clearly imply that the $\widetilde{\mathrm{E}} 2^{2} \mathrm{~A}_{1}$ state potential energy surface is highly repulsive in the region reached by the photoionization excitation process. There are no available theoretical calculations on the potential energy surface of this state. The details of intramolecular dynamics of this state must also await further high level theoretical exploration involving multimode coupled vibronic and anharmonic effects.

\section{F. Conclusions}


A high resolution photoelectron spectroscopy study has been performed on supersonic molecular beams of $\mathrm{H}_{2} \mathrm{CCO}$ and $\mathrm{D}_{2} \mathrm{CCO}$. The combination of high resolution and effective rotational cooling by supersonic expansion, as well as a least-squares fitting data reduction procedure, have enabled spectroscopic constants of much improved accuracy to be determined for the $\widetilde{\mathrm{X}} 1^{2} \mathrm{~B}_{1}, \widetilde{\mathrm{A}} 1^{2} \mathrm{~B}_{2}, \widetilde{\mathrm{B}} 2^{2} \mathrm{~B}_{1}, \widetilde{\mathrm{C}} 2^{2} \mathrm{~B}_{2}$, and $\widetilde{\mathrm{E}} 2^{2} \mathrm{~A}_{1}$ states. The complex nature and the strong overlap with other bands left the $\tilde{\mathrm{D}} 1^{2} \mathrm{~A}_{1}$ state much less well characterized. Most of the spectroscopic constants of the ionic states are obtained here for the first time. This should prompt further theoretical calculations to be performed on a high level. The discrepancies in the observed and calculated spectra in the first and second excited states for the ketene cations pointed out the need to improve the theoretical sophistication to match the currently available experimental results. The present studies on such a complex system as ketene represents a major challenge to the present level of theoretical sophistication to tackle the structure, spectroscopy and dynamics of polyatomic molecules and ions. The first excited state in this regard will be particularly interesting to follow the points implied from the present study are studied thoroughly in the near future by theoretical calculations. Multimode coupled vibronic calculations on the spectra, together with multidimensional calculations on the ionic potential energy surfaces, would be extremely important to push forward our understanding on the spectroscopy, structure, and dynamics of polyatomic ions.

Vibrational autocorrelation functions were calculated from the photoelectron bands for all but two electronic states of the ketene cations. The ground $\tilde{\mathrm{X}} 1^{2} \mathrm{~B}_{1}$ state correlation functions displayed oscillatory patterns, which were characteristic of the virtually undisplaced wave packets composed mainly of two oscillators. The wave packets oscillated with small amplitude around the initial position on the ionic potential energy surface with little spreading, indicated a fairly stable state in the Franck-Condon region. 
In the calculated $\tilde{\mathrm{A}} 1^{2} \mathrm{~B}_{2}$ state correlation functions, an ultra fast decay of the wave packets were observed. This effect was attributed largely to the possible predissociating nature of this state near the initially populated potential energy surface, which fervently awaits further theoretical characterization. The correlation functions of the $\widetilde{\mathrm{E}} 2^{2} \mathrm{~A}_{1}$ state showed ultra fast decay of the initial prepared wave packet on the ionic potential energy surface on a time scale of a few femtoseconds. This implied that dissociation and ultra fast intramolecular dynamic processes were among the important decay pathways for the initially prepared wave packet around the Franck-Condon region on the ionic potential energy surface for this state. 


\section{References:}

1. A. D. Baker, and D. W. Turner, Chem. Comm., 480(1969).

2. D. W. Turner, Phil. Trans. R. Soc., London Ser., A7, 268(1970).

3. D. W. Turner, A. D. Baker, and C. R. Brundle, Molecular Photoelectron Seectroscopy, John Wiley \& Sons, London (1970) p139.

4. D. Hall, J. P. Maier, and P. Rosmus, Chem. Phys., 373, 24(1977).

5. D. P. Chong, Theoretical Chim. Acta., 181, 50(1978).

6. K. Takeshita, J. Chem. Phys., 26(2), 1199(1992).

7. E. J. Heller, ibid, 68, 2066(1978).

8. E. J. Heller, E. B. Stechel, and M. J. Davis, ibid, 73(10), 4720(1980).

9. E. J. Heller, Acc. Chem. Res., 14, 368(1981).

10. E. J. Helller, R. Sundberg, and D. Tanner, J. Chem. Phys., 76, 1822(1982).

11. B. Layh-Nihaut, and J. C. Lorquet, ibid, 88(9), 5606(1988).

12. A. J. Lorquet, J. C. Lorquet, J. Delwiche, and M. J. Hubin-Franskin, ibid, 76(10), 4692(1982).

13. B. RuScic ibid, 85(7), 3776(1986).

14. F. Remacle, M. Desoute-Leconmte, and J. C. Lorquet, ibid, 21(7), 4155(1989).

15. J. E Pollard, D. J. Trevor, J. E Reutt, Y. T. Lee, and D. A. Shirley, J. Chem. Phys., 81(2), 5302(1984).

16. J. E. Reutt, L. S. Wang, J. E. Pollard, D. J. Trevor, Y. T. Lee, and D. A. Shirley, ibid, 84(6), 3022(1986).

17. J. E. Reutt, L. S. Wang, Y. T. Lee, and D. A. Shirley, ibid, 85(12), 6928(1986).

18. J. E. Pollard, D. J. Trevor, Y. T. Lee, and D. A. Shirley, Rev. Sci. Instrum., 52, 1837(1981).

19. J. E. Pollard, Ph.D. Thesis, Department of Chemistry, The University of California at Berkeley, 1982. 
20. J. E. Reutt, Ph.D. Thesis, Department of Chemistry, The University of California at Berkeley, 1986.

21. Williams and Hurd, J. Org. Chem., 5, 122(1940).

22. G. J. Fisher, A. F. Maclean, and A. W. Schwizer, J. Org. Chem., 1055(1952).

23. B. I. Sonobe, and R. N. Rosenfeld, JACS., 105, 7528(1983),

24. H. Bitto, D. R. Guyer, W. F. Polik, and C. B. Moore, Faraday Trans. Dis. Chem. Soc., 81, 149(1986).

25. I-Chia Chen, W. H. Green, Jr., and C. B. Moore, J. Chem. Phys., 89(1), 314(1988).

26. I-Chia Chen, and C. B. Moore, J. Phys. Chem., 24, 263,269(1990)

27. A. Lufthus, and P. H. Krupenie, J. Phys. Chem. Ref. Data, 6, 113(1977).

28. C. E. Moore, ed., Atsmic Energy Levels, Yol. I. (National Bureau of Standards, Washington D. C., 1958).

29. M. Born, and J. R. Oppenheimer, Ann. Phyz. (Lepzig), 84, 457-487(1927).

30. J. W. Rabalais, Principles of Ultraviolet Photoelectron Spectroscopy, John Wiley \& Sons, NY (1979).

31. H. Köppel, W. Domcke, and L. S. Cederbaum, Adv. Chem. Phys., Yol.LVII, p59-246(1984).

32. L. S. Cederbaum, and W. Domcke, J. Chem. Phys., 64(2), 603(1976).

33. W. Domcke, and L. S. Cederbaum,ibid, 64(2), 612(1976).

34. S. B. O'Neil, and W. P. Reinhardt, ibid, 69, 2126(1978).

35. E. Oran. Brighan, The Fast Fourier Transform, (Prentice-Hall, Englewood Cliffs), p164(1974).

36. J. I. Steinfeld, An Introduction to Modern Molecular Spectroscopy, 2nd. ed., The MIT Press, Cambridge, MA, p133(1985). 
37. G. Herzberg, Molecular_Spectra and Molecular_Structures, L_Spectra of Diatomic Molecules, Van Norstrand Reinhold Com., NY, P93(1950).

38. J. L. Duncan, A. M. Fergurnn, J. Harpper, and K. H. Tonge, J. Mol. Spectr., 125, 196-213(1987)

39. J. L. Duncan, A. M. Ferguson, J. Harpper, and K. H. Tonge, ibid, 122, 72 93(1987).

40. C. B. Moore, and G. Pimentel, J. Chem. Phys., 38(12), 2816(1963).

41. L. S. Wang, J. E. Reutt, Y. T. Lee, and D. A. Shirley, J. Elect. Spectr. and Related Phenomena, 47, 167-186(1988).

42. W. D. Allen, and H. F. Schaeffer III, J. Chem. Phys., 84, 2212(1986).

43. W. D. Allen, and H. F. Schaeffer III, ibid, 87, 7076(1987).

44. W. D. Allen, and H. F. Schaeffer III, ibid, 89, 2329(1988).

45. R. A. Marcus, Science, Yol. 256, p1523, 12 June1992.

46. E. R. Lovejoy, S. K. Kim, and C. B. Moore, ibid, Yol. 256, p1541(1992). 


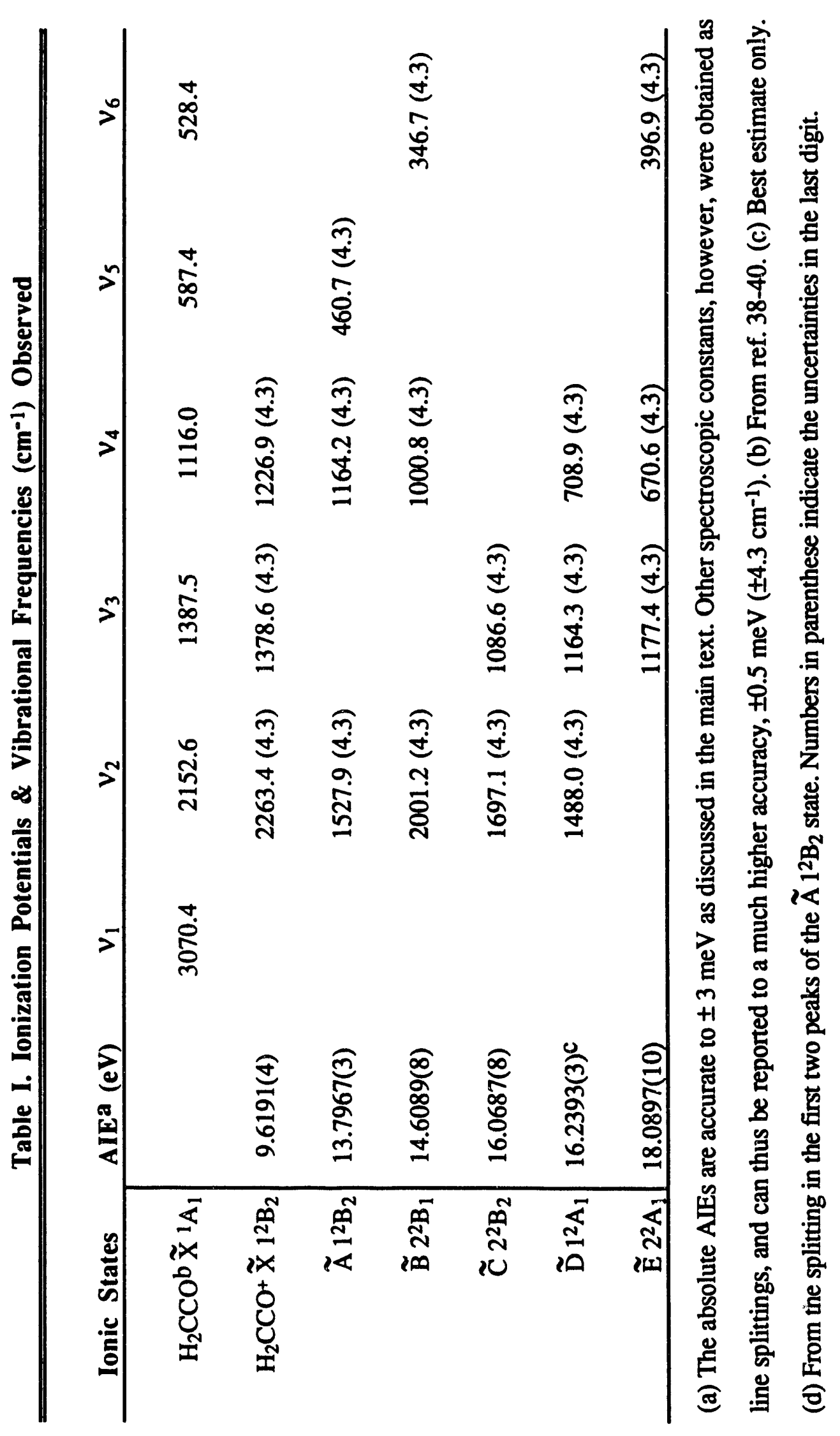

139 


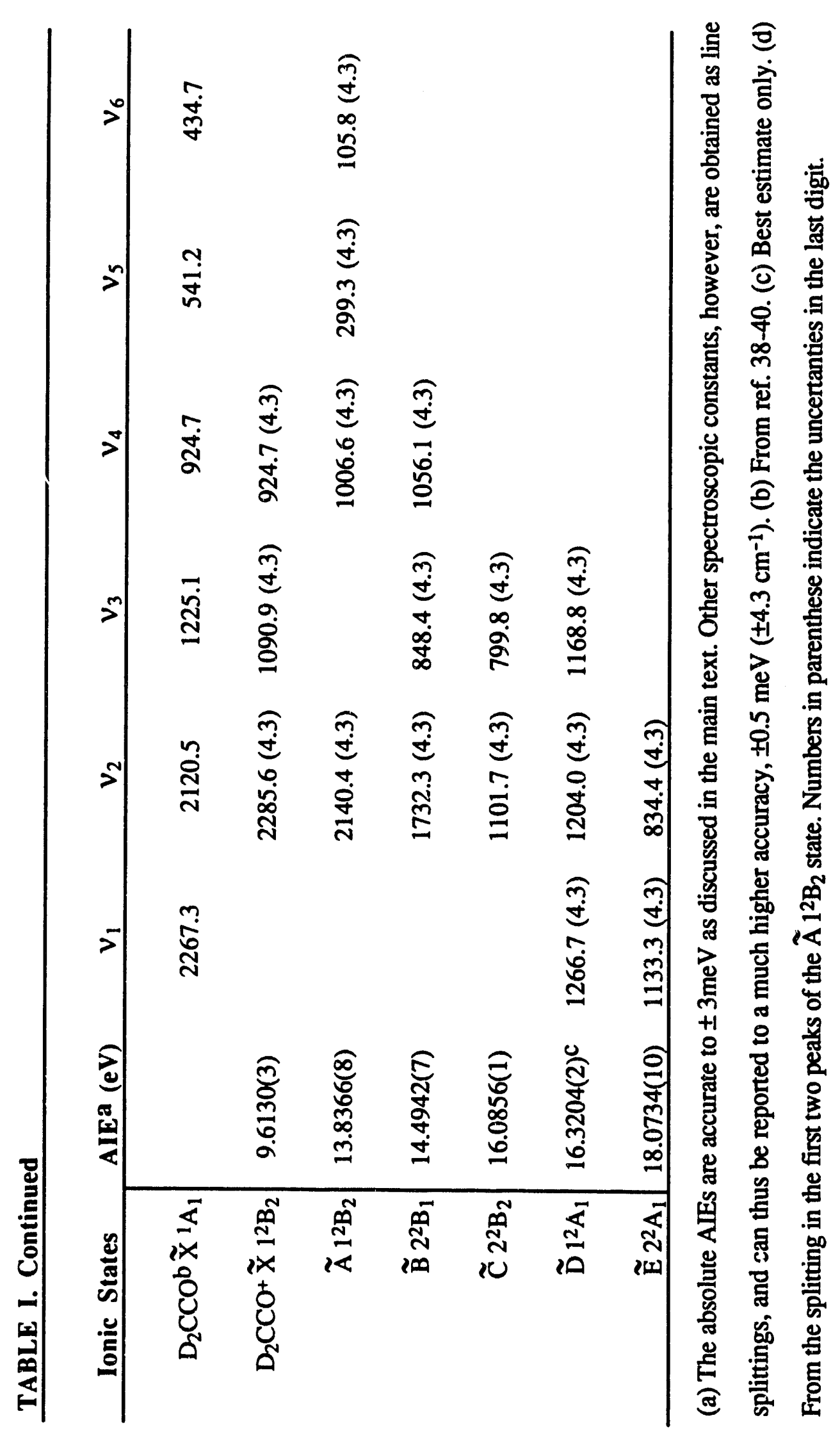


TABLE II. Vibrational Levels of the $\widetilde{\mathbf{X}} \mathbf{1 2}^{2} \mathrm{~B}_{1}$ State

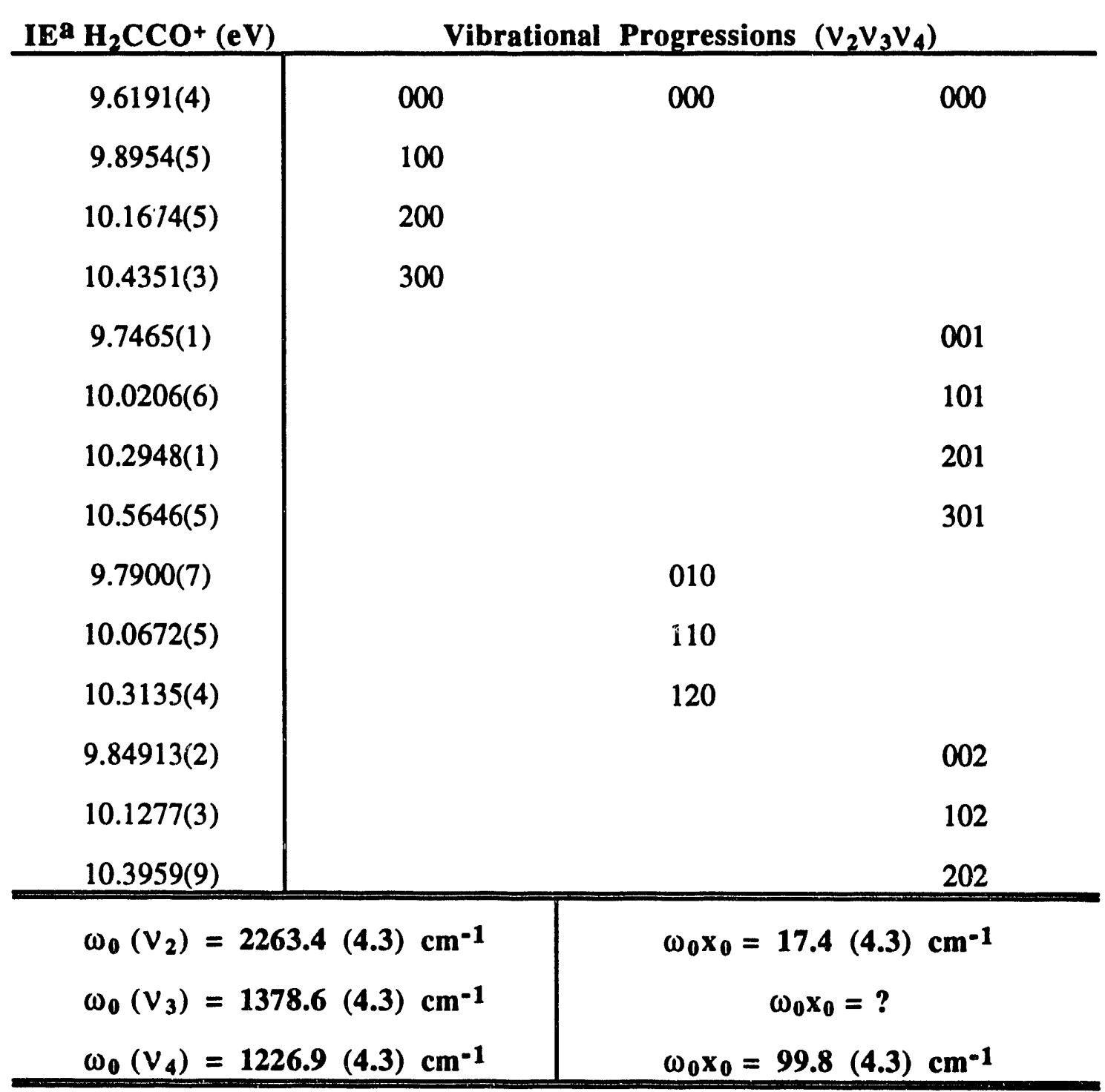

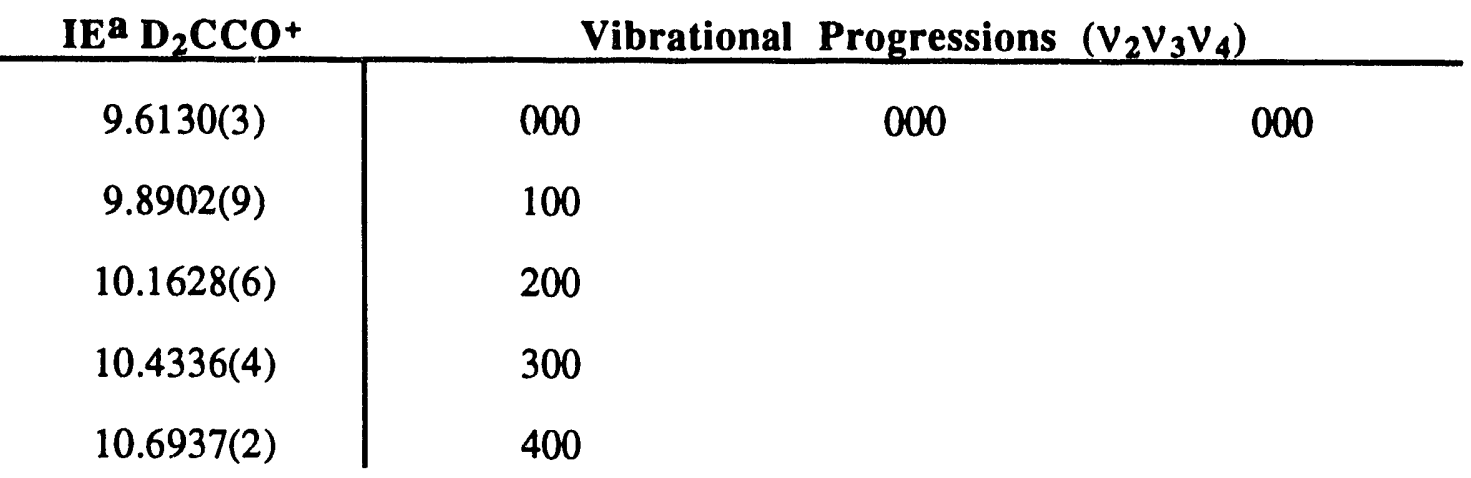


TABLE II. Continued

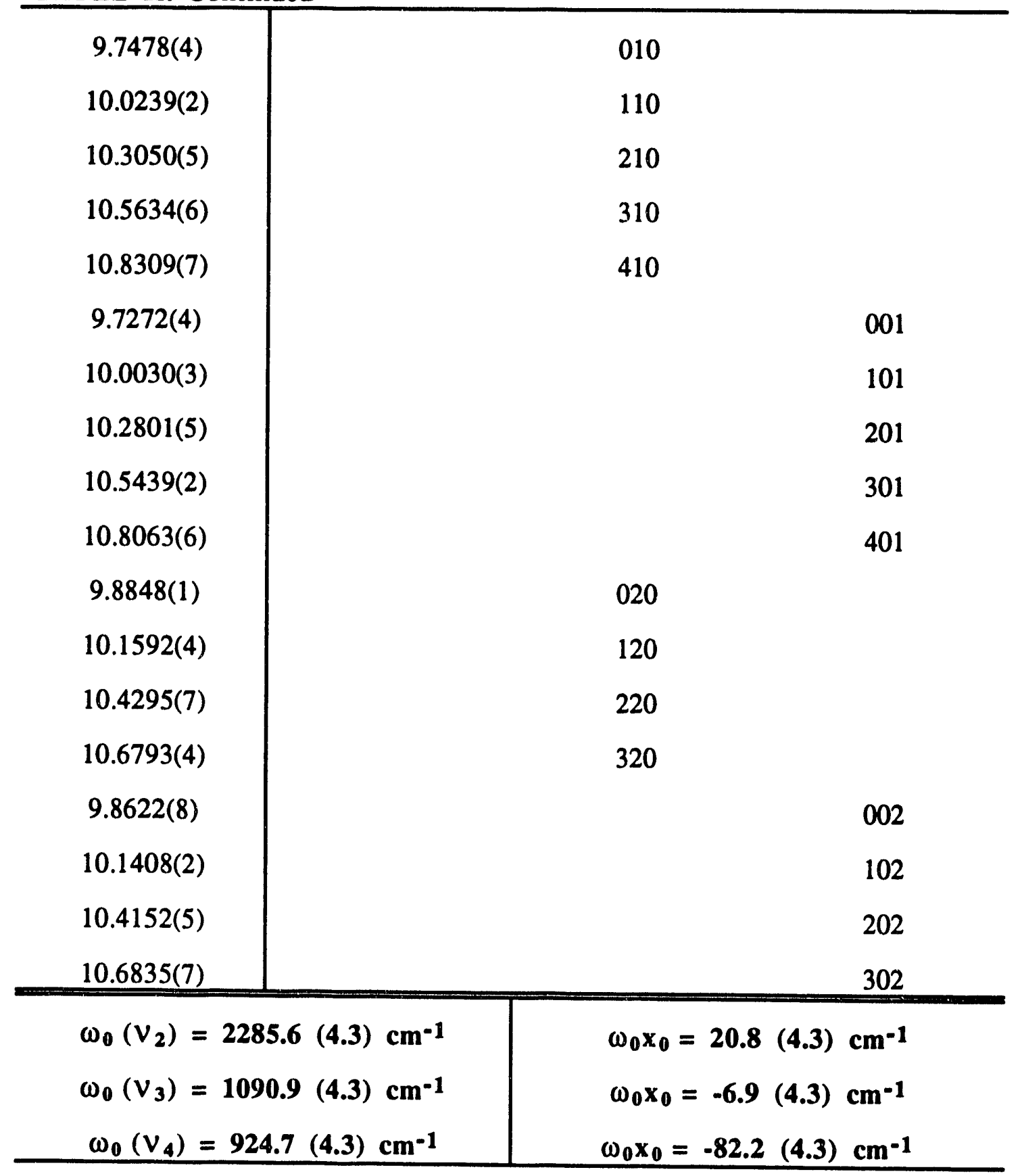

a. See footnote in TABLE I. 
TABLE III. Vibrational Levels of the $\widetilde{A} 1^{2} B_{2}$ State

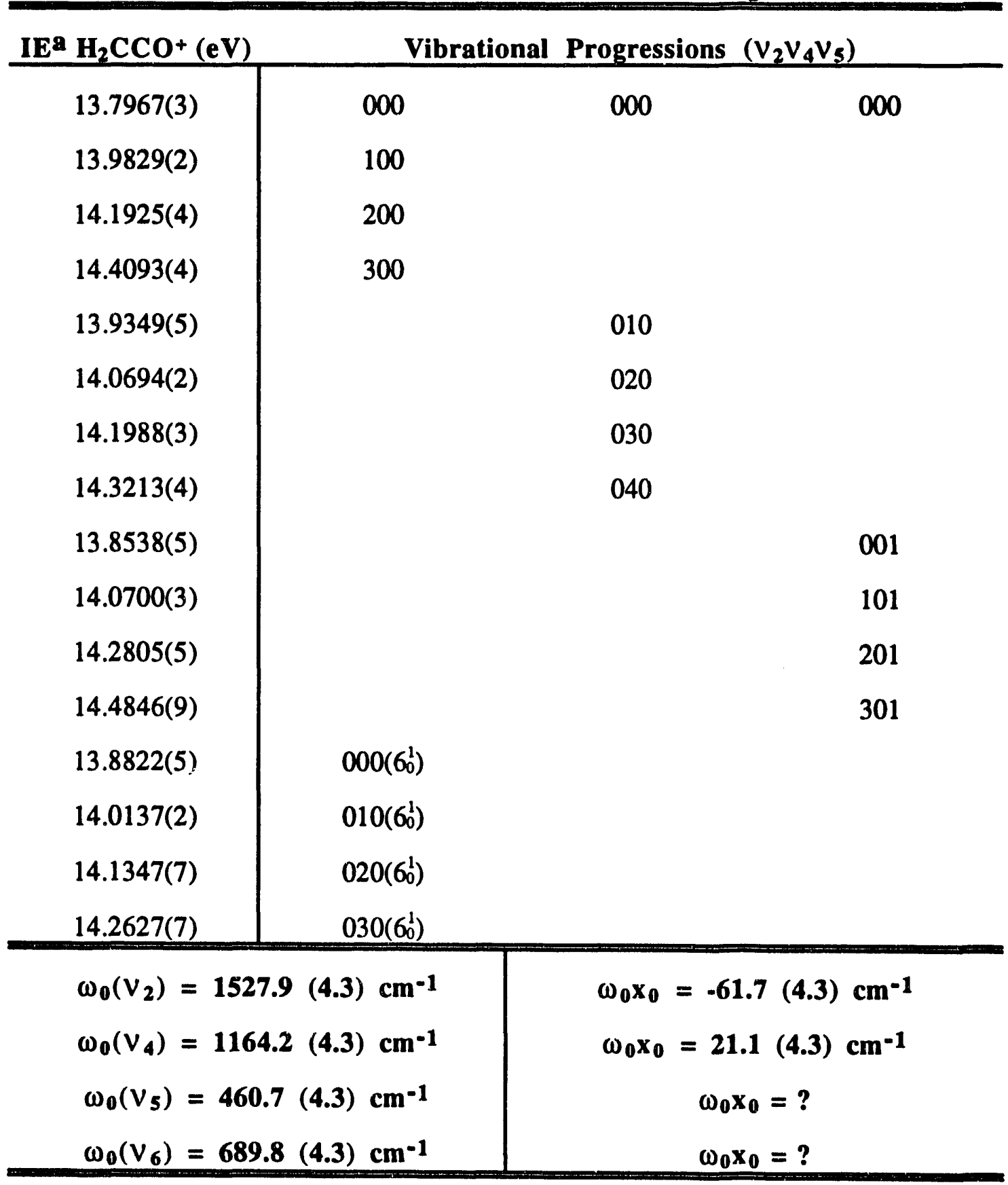

$\mathrm{IEa} \mathrm{D}_{2} \mathrm{CCO}^{+}(\mathrm{eV})$

$13.8366(8)$

$13.9653(2)$
Vibrational Progressions $\left(V_{2} V_{4} V_{5}\right)$

000

000

000

010 
TABLE III. Continued

\begin{tabular}{|c|c|c|}
\hline $14.0939(7)$ & & 020 \\
\hline $14.2337(4)$ & & 030 \\
\hline $14.3648(9)$ & & 040 \\
\hline $14.5092(8)$ & & 050 \\
\hline $13.8737(9)$ & & 001 \\
\hline $14.1385(2)$ & & 101 \\
\hline $14.4019(7)$ & & 201 \\
\hline $13.9331(6)$ & & $000\left(6_{0}^{1}\right)$ \\
\hline $14.0605(7)$ & & $010\left(6_{0}^{1}\right)$ \\
\hline $14.1879(7)$ & & $020\left(6_{0}^{1}\right)$ \\
\hline $14.3188(3)$ & & $030\left(6_{0}^{1}\right)$ \\
\hline $14.0049(1)$ & $001\left(6_{0}^{1}\right)$ & \\
\hline $14.2337(4)$ & $101\left(6_{0}^{1}\right)$ & \\
\hline $14.4625(8)$ & $201\left(6_{0}^{1}\right)$ & \\
\hline \multicolumn{2}{|c|}{$\begin{array}{l}\omega_{0}\left(v_{2}\right)=2140.4(4.3) \mathrm{cm}^{-1} \\
\omega_{0}\left(v_{4}\right)=1006.7(4.3) \mathrm{cm}^{-1} \\
\omega_{0}\left(v_{5}\right)=299.3(4.3) \mathrm{cm}^{-1} \\
\omega_{0}\left(v_{6}\right)=105.8(4.3) \mathrm{cm}^{-1}\end{array}$} & $\begin{array}{c}\omega_{0} x_{0}=5.2(4.3) \mathrm{cm}^{-1} \\
\omega_{0} x_{0}=-12.8(4.3) \mathrm{cm}^{-1} \\
\omega_{0} x_{0}=? \\
\omega_{0} x_{0}=?\end{array}$ \\
\hline
\end{tabular}

a. See footnote in TABLE I. 
TABLE IV. Vibrational Levels of the $\widetilde{B} 2^{2} B_{1}$ State

\begin{tabular}{|c|c|c|c|}
\hline $\mathrm{IE}^{\mathrm{a}} \mathrm{H}_{2} \mathrm{CCO}^{+}(\mathrm{eV})$ & Vibrational & Progressions & $\left(V_{2} V_{4} V_{6}\right)$ \\
\hline $14.6089(8)$ & 000 & 000 & 000 \\
\hline $14.7320(6)$ & & 010 & \\
\hline $14.8531(9)$ & $(100)^{b}$ & 020 & \\
\hline $14.9743(2)$ & & 030 & \\
\hline $15.0934(9)$ & $(200)^{b}$ & 040 & \\
\hline $15.2107(1)$ & & 050 & \\
\hline $15.3259(8)$ & & 060 & \\
\hline $15.44191(1)$ & & 070 & \\
\hline $15.5662(9)$ & & 080 & \\
\hline $15.6721(4)$ & & 090 & \\
\hline $15.7820(9)$ & & $0(10) 0$ & \\
\hline $14.6519(6)$ & & & 001 \\
\hline $14.7672(3)$ & & & 011 \\
\hline $14.8942(2)$ & & & 021 \\
\hline $15.0114(4)$ & & & 031 \\
\hline $15.1403(8)$ & & & 041 \\
\hline $15.2572(8)$ & & & 051 \\
\hline $15.3670(1)$ & & & 061 \\
\hline $15.4805(1)$ & & & 071 \\
\hline $15.5897(3)$ & & & 081 \\
\hline $15.7004(1)$ & & & 091 \\
\hline $15.8072(2)$ & & & $0(10) 1$ \\
\hline
\end{tabular}


TABLE IV. Continued

\begin{tabular}{|c|c|c|c|}
\hline $\mathrm{IE}^{\mathrm{a}} \mathrm{D}_{2} \mathrm{CCO}^{+}(\mathrm{eV})$ & Vibrational & Progressions & $\left(V_{2} V_{3} V_{4}\right)$ \\
\hline $14.6106(5)$ & 000 & 000 & 000 \\
\hline $14.7404(6)$ & & & 001 \\
\hline $14.8725(1)$ & & & 002 \\
\hline $15.0023(2)$ & & & 003 \\
\hline $15.1327(7)$ & & & 004 \\
\hline $14.7158(4)$ & & 010 & \\
\hline $14.8478(9)$ & & 011 & \\
\hline $14.9732(3)$ & & 012 & \\
\hline $15.1052(8)$ & & 013 & \\
\hline $15.2328(5)$ & & 014 & \\
\hline $15.3787(2)$ & & 015 & \\
\hline $15.5169(7)$ & & 016 & \\
\hline $14.8255(1)$ & 100 & & \\
\hline $14.9530(8)$ & 101 & & \\
\hline $15.0784(2)$ & 102 & & \\
\hline $15.2059(9)$ & 103 & & \\
\hline $15.3358(0)$ & 104 & & \\
\hline $15.4708(9)$ & 105 & & \\
\hline $15.0653(6)$ & & & 110 \\
\hline $15.1836(1)$ & & & 111 \\
\hline $15.3089(5)$ & & & 112 \\
\hline $15.4298(0)$ & & & 113 \\
\hline $15.5444(0)$ & & & 114 \\
\hline
\end{tabular}


TABLE IV. Continued

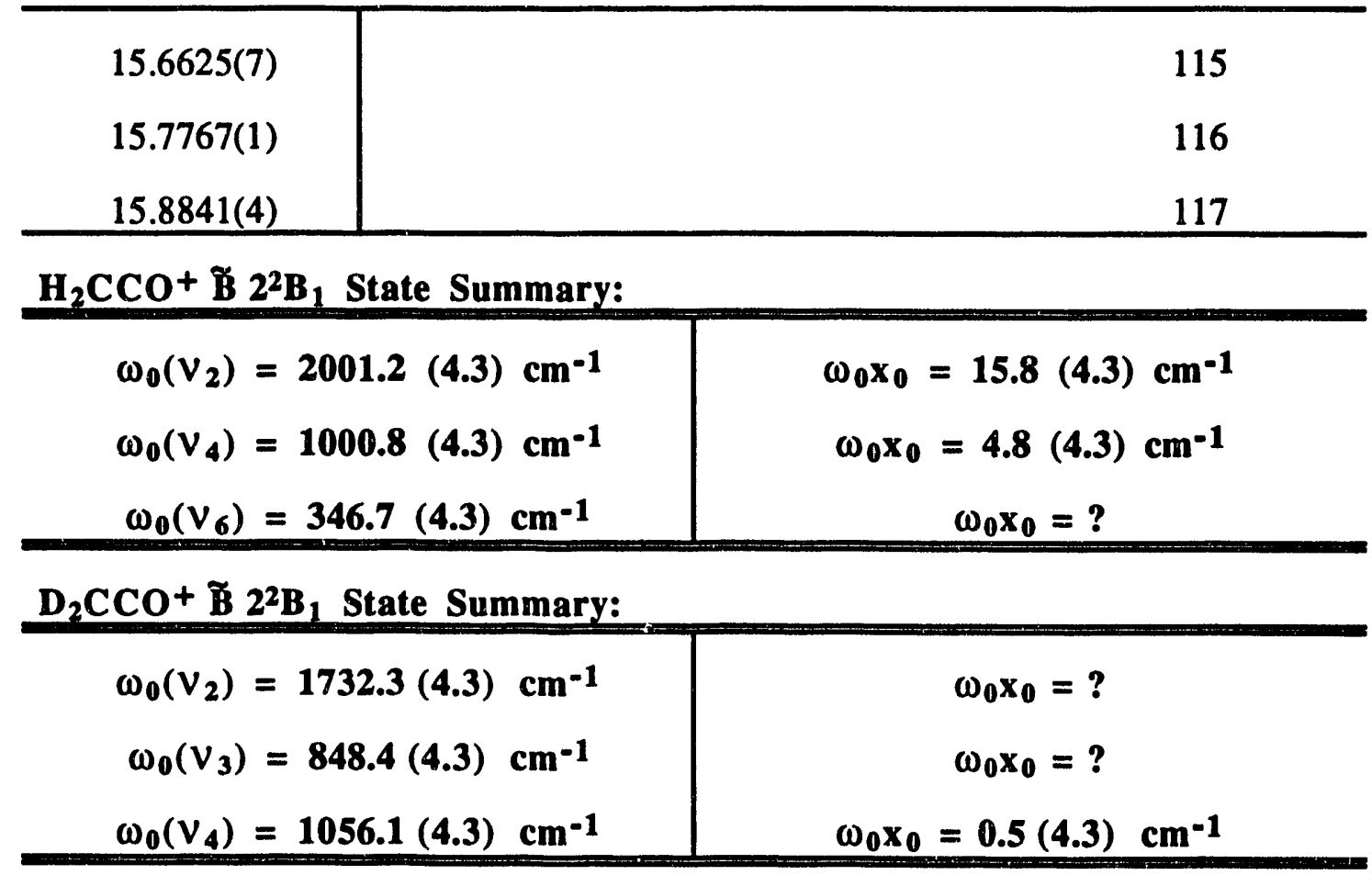

(a) See footnote in TABLE I. (b) Fermi resonances peaks. 
TABLE V. Vibrational Levels of the $\widetilde{C} 2^{2} B_{2}$ State

\begin{tabular}{|c|c|c|c|}
\hline $\mathrm{IE}^{\mathrm{a}} \mathrm{H}_{2} \mathrm{CCO}^{+}(\mathrm{eV})$ & \multicolumn{3}{|c|}{ Vibrational Progressions $\left(V_{2} V_{3}\right)$} \\
\hline $16.0687(8)$ & 00 & 00 & 00 \\
\hline $16.1981(6)$ & & 01 & \\
\hline $16.3257(5)$ & & 02 & \\
\hline $16.4567(8)$ & & 03 & \\
\hline $16.5862(2)$ & & 04 & \\
\hline $16.7155(5)$ & & 05 & \\
\hline $16.8365(8)$ & & 06 & \\
\hline $16.9512(9)$ & & 07 & \\
\hline $17.0746(8)$ & & 08 & \\
\hline 16.06878$)$ & 00 & & \\
\hline $16.2790(2)$ & 10 & & \\
\hline $16.4915(5)$ & 20 & & \\
\hline $16.7021(8)$ & 30 & & \\
\hline $16.9121(4)$ & 40 & & \\
\hline $17.1280(4)$ & 50 & & \\
\hline $17.3381(9)$ & 60 & & \\
\hline $16.1721(2)$ & & & 01 \\
\hline $16.3625(6)$ & & & 11 \\
\hline $16.5557(4)$ & & & 21 \\
\hline $16.7443(1)$ & & & 31 \\
\hline $16.9376(7)$ & & & 41 \\
\hline $\begin{array}{l}\omega_{0}\left(v_{2}\right)=169 \\
\omega_{0}\left(v_{3}\right)=108\end{array}$ & $\begin{array}{l}\mathrm{cm}^{-1} \\
\mathrm{~cm}^{-1} \\
\end{array}$ & $\begin{aligned} \omega_{0} x_{0} & =-1.4 \\
\omega_{0} x_{0} & =7.8\end{aligned}$ & $\begin{array}{l}\text { (4.3) } \mathrm{cm}^{-1} \\
\text { (4.3) } \mathrm{cm}^{-1}\end{array}$ \\
\hline
\end{tabular}


Table V. Continued

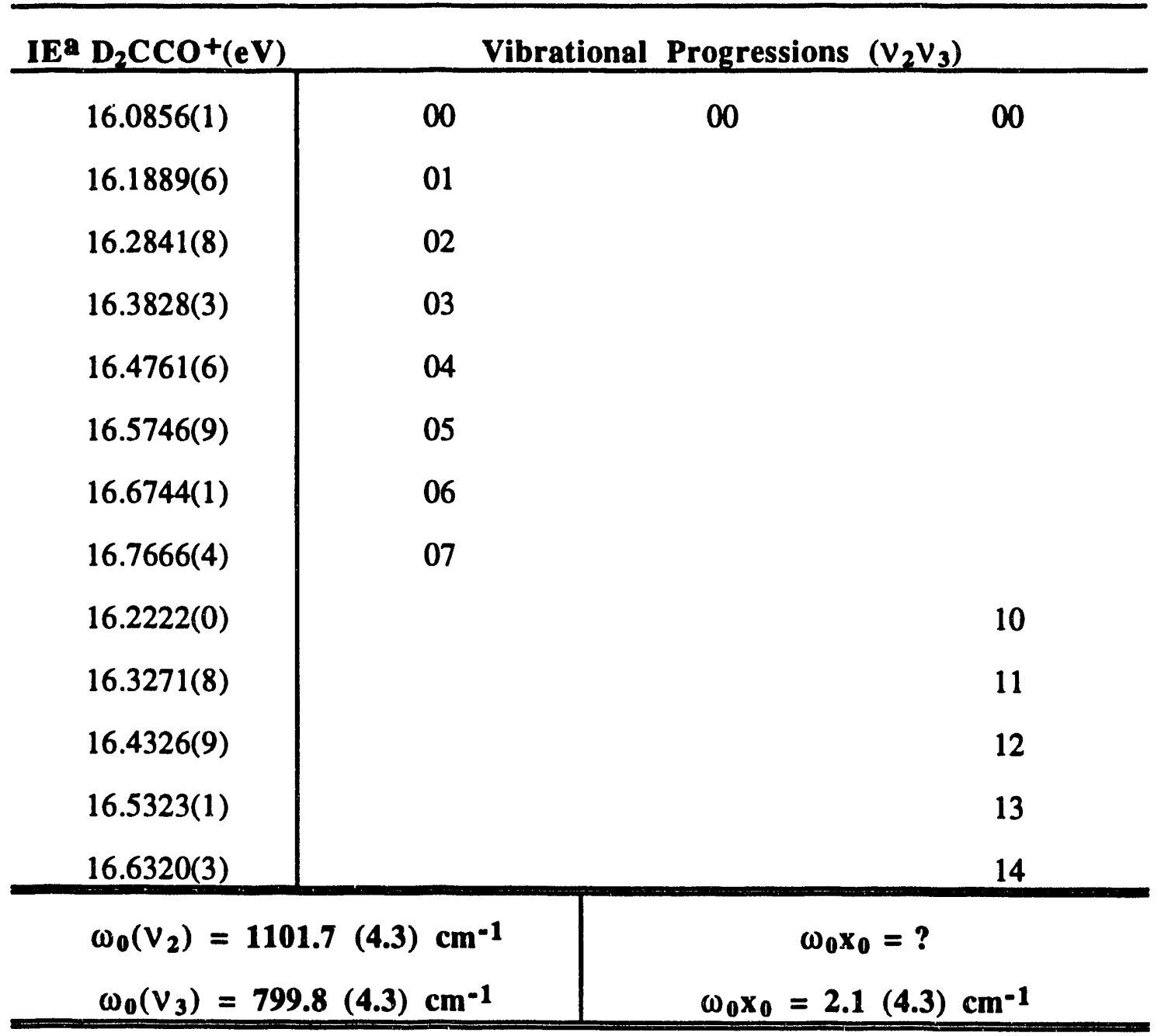

a. See footnote in TABLE I. 
TABLE VI. Vibrational Levels of the $\tilde{D} 1^{2} A_{1}$ State

\begin{tabular}{|c|c|c|c|}
\hline $\mathrm{IE}^{\mathrm{a}} \mathrm{H}_{2} \mathrm{CCO}^{+}(\mathrm{eV})$ & Vibrational & Progressions & $\left(V_{2} V_{3} V_{4}\right)$ \\
\hline $16.2393(3)$ & & 000 & \\
\hline $16.4438(5)$ & 100 & & \\
\hline $16.6267(3)$ & 200 & & \\
\hline $16.8235(4)$ & 300 & & \\
\hline $17.0279(0)$ & 400 & & \\
\hline $17.2422(6)$ & 500 & & \\
\hline $16.4281(1)$ & & & 100 \\
\hline $16.5067(7)$ & & & 101 \\
\hline $16.5913(4)$ & & & 102 \\
\hline $16.6798(3)$ & & & 103 \\
\hline $16.7624(2)$ & & & 104 \\
\hline $16.8450(2)$ & & & 105 \\
\hline $16.9295(8)$ & & & 106 \\
\hline $17.0121(8)$ & & & 107 \\
\hline $17.0869(0)$ & & & 108 \\
\hline $17.1596(6)$ & & & 109 \\
\hline $16.5008(8)$ & & 101 & \\
\hline $16.6346(0)$ & & 111 & \\
\hline $16.7781(5)$ & & 121 & \\
\hline $16.9118(8)$ & & 131 & \\
\hline $17.0495(4)$ & & 141 & \\
\hline $17.1793(3)$ & & 151 & \\
\hline $17.3012(5)$ & & 161 & \\
\hline
\end{tabular}


TABLE VI. Continued

\begin{tabular}{|c|c|c|c|}
\hline $\mathrm{IE}^{\mathrm{a}} \mathrm{D}_{2} \mathrm{CCO}^{+}(\mathrm{eV})$ & Vibrational & Progressions & $\left(V_{1} V_{2} V_{3}\right)$ \\
\hline $16.3204(2)$ & 000 & 000 & 000 \\
\hline $16.4625(1)$ & 001 & & \\
\hline $16.5971(3)$ & 002 & & \\
\hline $16.7367(3)$ & 003 & & \\
\hline $16.8663(6)$ & 004 & & \\
\hline $16.9934(9)$ & 005 & & \\
\hline $16.477 A_{5}(7)$ & & & 100 \\
\hline $16.6295(4)$ & & & 110 \\
\hline $16.7766(1)$ & & & 120 \\
\hline $16.9162(1)$ & & & 130 \\
\hline $17.0508(3)$ & & & 140 \\
\hline $17.1954(1)$ & & & 150 \\
\hline $17.3399(9)$ & & & 160 \\
\hline $17.4671(3)$ & & & 170 \\
\hline
\end{tabular}

$\mathrm{H}_{2} \mathrm{CCO}^{+} \widetilde{\mathrm{D}} \mathbf{1}^{2} \mathrm{~A}_{1}$ State Summary:

\begin{tabular}{l|l}
$\omega_{0}\left(v_{2}\right)=1488.0(4.3) \mathrm{cm}^{-1}$ & $\omega_{0} x_{0}=-19.5(4.3) \mathrm{cm}^{-1}$ \\
$\omega_{0}\left(V_{3}\right)=1164.3(4.3) \mathrm{cm}^{-1}$ & $\omega_{0} x_{0}=11.5(4.3) \mathrm{cm}^{-1}$ \\
$\omega_{0}\left(V_{4}\right)=708.9(4.3) \mathrm{cm}^{-1}$ & $\omega_{0} x_{0}=4.5(4.3) \mathrm{cm}^{-1}$
\end{tabular}

$\mathrm{D}_{2} \mathrm{CCO}+\widetilde{\mathrm{D}} \mathbf{1 2}_{1} \mathrm{~A}_{1}$ State Summary:

$$
\begin{array}{c|c}
\omega_{0}\left(v_{1}\right)=1266.7(4.3) \mathrm{cm}^{-1} & \omega_{0} x_{0}=? \\
\omega_{0}\left(v_{2}\right)=1204.9(4.3) \mathrm{cm}^{-1} & \omega_{0} x_{0}=8.2(4.3) \mathrm{cm}^{-1} \\
\omega_{0}\left(v_{3}\right)=1168.8(4.3) \mathrm{cm}^{-1} & \omega_{0} x_{0}=13.6(4.3) \mathrm{cm}^{-1}
\end{array}
$$


TABLE VII. Vibrational Levels of the E $2^{2} A_{1}$ State

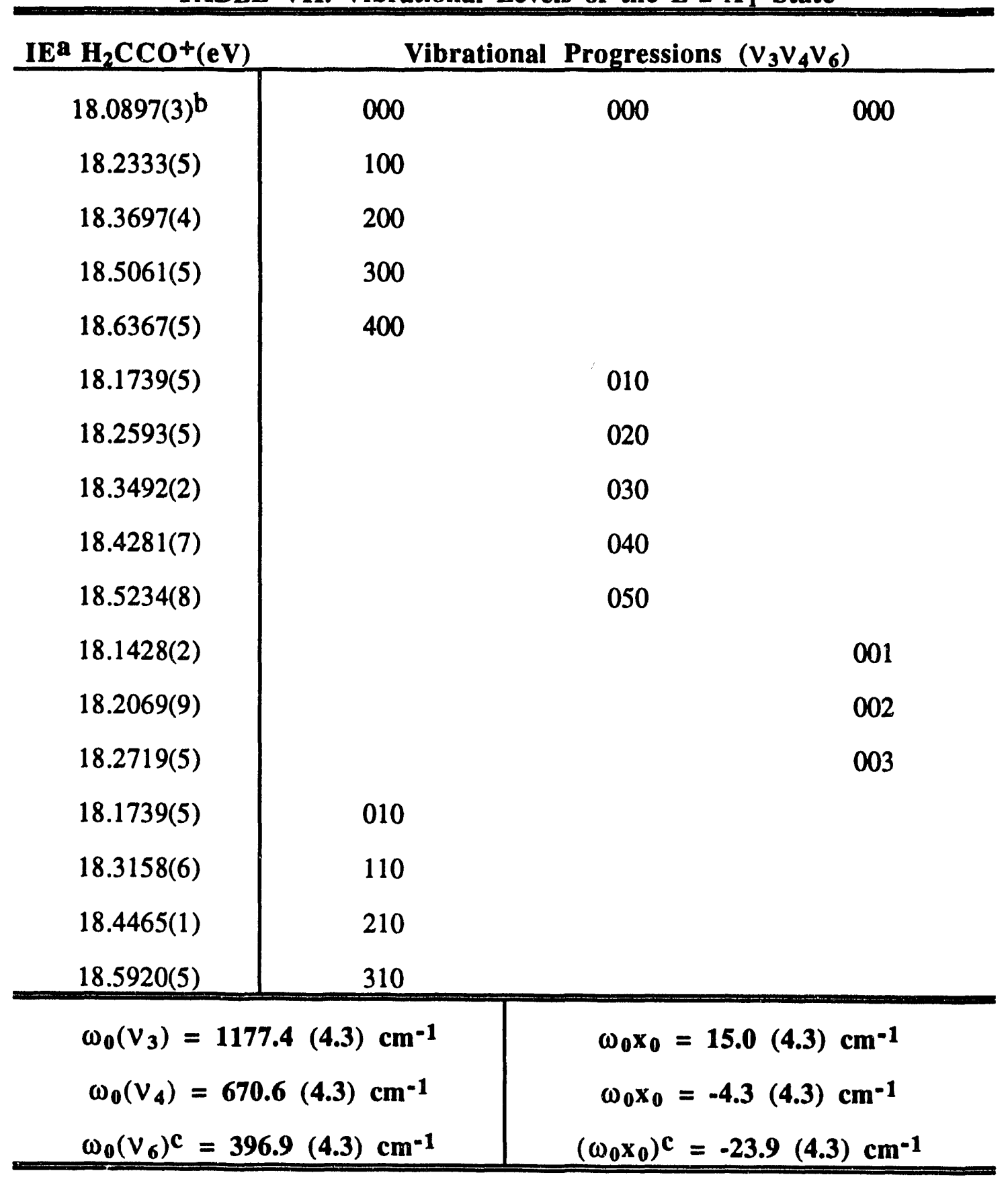


TABLE VII. Continued

\begin{tabular}{c|cc}
\hline \multicolumn{2}{c}{ IEa $\mathrm{D}_{2} \mathrm{CO}+(\mathrm{eV})$} & \multicolumn{2}{c}{ Vibrational Levels $\left(\mathrm{V}_{1} \mathrm{~V}_{2}\right)$} \\
\hline $18.0734(5)^{\mathrm{b}}$ & 00 & 00 \\
$18.2101(4)$ & 10 & \\
$-18.3456(1)$ & 20 & 01 \\
$18.4761(5)$ & 30 & 11 \\
$18.1768(9)$ & & 21 \\
$18.3197(5)$ & \multicolumn{2}{c}{} \\
$18.4564(5)$ & $\omega_{0} \mathrm{x}_{0}=12.4(4.3) \mathrm{cm}^{-1}$ \\
\hline$\omega_{0}\left(V_{1}\right)=1133.3(4.3) \mathrm{cm}^{-1}$ & $\omega_{0} \mathrm{x}_{0}=?$ \\
\hline
\end{tabular}

(a) See Table I. footnote. (b) This is only the best estimate $\pm 10 \mathrm{meV}$. (c) Tentaive values. 


\section{Figure Captions:}

Figure 1. The full spectrum of ketene with a resolution of $11.5 \mathrm{meV}$ FWHM, the result of five sequential scans each lasting about two hours. Each scan was repeated four times to enhance statistics. The designation of the ionic states assumes that the ions have $\mathrm{C}_{2 \mathrm{~V}}$ symmetry.

Figure 2. The full spectrum of $d_{2}$-ketene with a resolution of $11.5 \mathrm{meV}$ FWHM, the result of five sequential scans each lasting about two hours. Each scan was repeated four times to enhance statistics. The designation of the ionic states assumes that the ions have $\mathrm{C}_{2} \mathrm{~V}$ symmetry.

Figure 3. The photoelectron spectrum of the $\widetilde{\mathrm{X}} 1^{2} \mathrm{~B}_{1}$ state of ketene, with a resolution of $11.5 \mathrm{meV}$ FWHM. The vibrational progressions are labeled according to the $\mathrm{C}_{2 \mathrm{v}}$ geometry and $2_{0}^{\mathrm{n}}$ stands for the transition of

$$
M^{+}\left(V_{2}=n\right)+e \leftarrow M\left(V_{2}=0\right)+\hbar \omega
$$

following standard spectroscopic notations.

Figure 4. The photoelectron spectrum of the $\widetilde{X} 1^{2} B_{1}$ state of $d_{2}$-ketene, with a resolution of $11.5 \mathrm{meV}$ FWHM. The vibrational progressions are labeled according to the $C_{2 v}$ geometry, and notations are the same as in the $\tilde{X}$ $1^{2} B_{1}$ state of ketene.

Figure 5. The vibrational autocorrelation function of the $\widetilde{\mathrm{X}}{ }^{2}{ }^{2} \mathrm{~B}_{1}$ state of ketene after all corrections were made. The beat pattern results from the phase relationship of a stable two-mode anharmonic oscillator system, with $v_{2}$ $\geq 2 v_{3}, 2 v_{4}$. The shallow minima in the correlation function are characteristics of the wave packet prepared through a predominately 
adiabatic transition. An essentially non-bonding electron is ejected, and the initially prepared wave packet is localized around the minimum of the upper potential energy surface. It weakly oscillates around this region, retaining a high level of correlation at all time.

Figure 6. The vibrational autocorrelation function of the $\tilde{\mathrm{X}} 1^{2} \mathrm{~B}_{1}$ state of $\mathrm{d}_{2}$-ketene after all corrections were made. Essentially the same behavior is observed as in the $\widetilde{\mathrm{X}}{ }^{2} \mathrm{~B}_{1}$ state of ketene.

Figure 7. The vibrational autocorrelation functions of the $\widetilde{\mathrm{X}} 1^{2} \mathrm{~B}_{1}$ states of ketene and $d_{2}$-ketene. The solid line is ketene and the dotted line is $d_{2}$-ketene. Note the isotope effect at longer times.

Figure 8. The photoelectron spectrum of the $\tilde{\mathrm{A}} 1^{2} \mathrm{~B}_{2}$ and $\widetilde{\mathrm{B}} 2^{2} \mathrm{~B}_{1}$ states of ketene with a resolution of $11.5 \mathrm{meV}$ FWHM. Note the fine vibrational progressions in the $\tilde{\mathrm{A}} 1^{2} \mathrm{~B}_{2}$ state were resolved here for the first time. The doublet-like structure in the $\tilde{\mathrm{A}} 1^{2} \mathrm{~B}_{2}$ was due to the excitation of a 'soft' mode, here designated as $V_{5}$. Also, the weaker progression in the $\widetilde{\mathrm{B}}{ }^{2} \mathrm{~B}_{1}$ state was due to the excitation of a 'soft' mode, here designated as $v_{6}$, together with the main excitation of $v_{4}$ mode.

Figure 9. The photoelectron spectrum of the $\widetilde{A} 1^{2} B_{2}$ and $\widetilde{B} 2^{2} B_{1}$ states of $d_{2}$-ketene with a resolution of $11.5 \mathrm{meV}$ FWHM. Comparing with the spectrum of ketene, a strong isotope effect on the vibrational fine structures is observed.

Figure 10. The vibrational correlation function calculated for the $\tilde{\mathrm{A}} 1^{2} \mathrm{~B}_{2}$ state of ketene after digitally removing the contributions from the $\widetilde{B} 2^{2} B_{1}$ state, and making all corrections. The most striking feature of the correlation function is the 
loss of correlation strength on an ultra fast time scale of a few fs. This implies that ketene cations were prepared on the repulsive side of the upper potential energy surface, and were subject to predissociation and other ultra fast intramolecular dynamic processes.

Figure 11. The vibrational correlation function calculated for the $\tilde{\mathrm{A}} \mathbf{1}^{2} \mathrm{~B}_{2}$ state of $\mathrm{d}_{2}$ ketene after digitally removing the contributions from the $\widetilde{\mathrm{B}} 2^{2} \mathrm{~B}_{1}$ state, and making all corrections.

Figure 12. The vibrational correlation function calculated for the $\widetilde{\mathrm{B}} 2^{2} \mathrm{~B}_{1}$ state of ketene after digitally removing the contributions from the $\widetilde{\mathrm{A}} 1^{2} \mathrm{~B}_{2}$ state, and making all corrections. The deep valleys in the correlation function indicate a displaced wave packet from the upper potential energy surface (PES) minimum.

Figure 13. The vibrational correlation function calculated for the $\widetilde{B} 2^{2} B_{1}$ state of $d_{2}$ ketene after digitally removing the contributions from the $\tilde{\mathrm{A}} 1^{2} \mathrm{~B}_{2}$ state, and making all corrections. The loss of correlation strength beyond $74 \mathrm{fs}$ was attributed to the spreading of the wave packet due to anharmonicity and the excitation of additional vibrational modes.

Figure 14. The photoelectron spectrum of the $\widetilde{\mathrm{C}} 2^{2} \mathrm{~B}_{2}$ and $\tilde{\mathrm{D}} 1^{2} \mathrm{~A}_{1}$ states of ketene, with a resolution of $11.5 \mathrm{meV}$ FWHM. The major vibrational progression was attributed to the $\widetilde{\mathrm{C}} 2^{2} \mathrm{~B}_{2}$ state.

Figure 15. The photoelectron spectrum of the $\widetilde{C} 2^{2} B_{2}$ and $\widetilde{D} 1^{2} A_{1}$ states of $d_{2}$-ketene with a resolution of $11.5 \mathrm{meV}$ FWHM. The major vibrational progression was attributed to the $\tilde{\mathrm{C}} 2^{2} \mathrm{~B}_{2}$ state. 
Figure 16. The photoelectron spectrum of the $\tilde{\mathrm{E}} 2^{2} \mathrm{~A}_{1}$ state of ketene with a resolution of $11.5 \mathrm{meV}$ FWHM. The very broad adiabatic peak and the asymmetric line shape imply that ketene cations in the Franck-Condon part of the PES are subject to lifetime broadening effects.

Figure 17. The photoelectron spectrum of the $\tilde{\mathrm{E}} 2^{2} \mathrm{~A}_{1}$ state of $\mathrm{d}_{2}$-ketene with a resolution of $11.5 \mathrm{meV}$ FWHM. The very broad adiabatic peak and the asymmetric line shape imply that this state in the Franck-Condon region is subject to lifetime broadening.

Figure 18. The vibrational correlation function calculated for the $\tilde{\mathrm{E}} 2^{2} \mathrm{~A}_{1}$ state of ketene after all corrections were made. The ultra fast decay of the correlation function implies that ultra fast intramolecular processes dominate the decay of the ketene cations in the Franck-Condon part of the PES.

Figure 19. The vibrational correlation function calculated for the $\tilde{E} 2^{2} A_{1}$ state of $d_{2}$ ketene after all corrections were made. The ultra fast decay of the correlation function implies that ultra fast intramolecular processes dominate the decay of the ketene cations in the Franck-Condon part of the PES. 


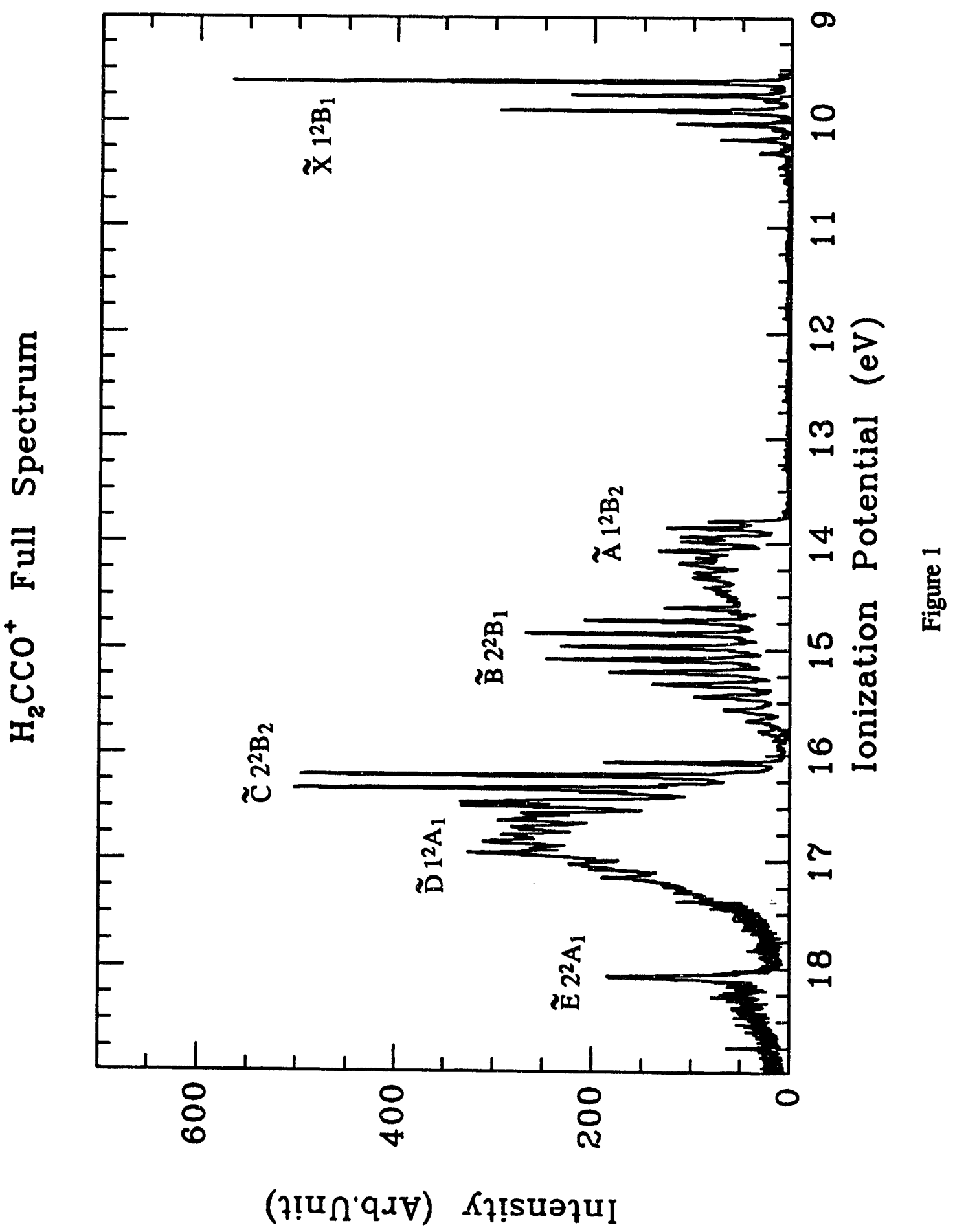




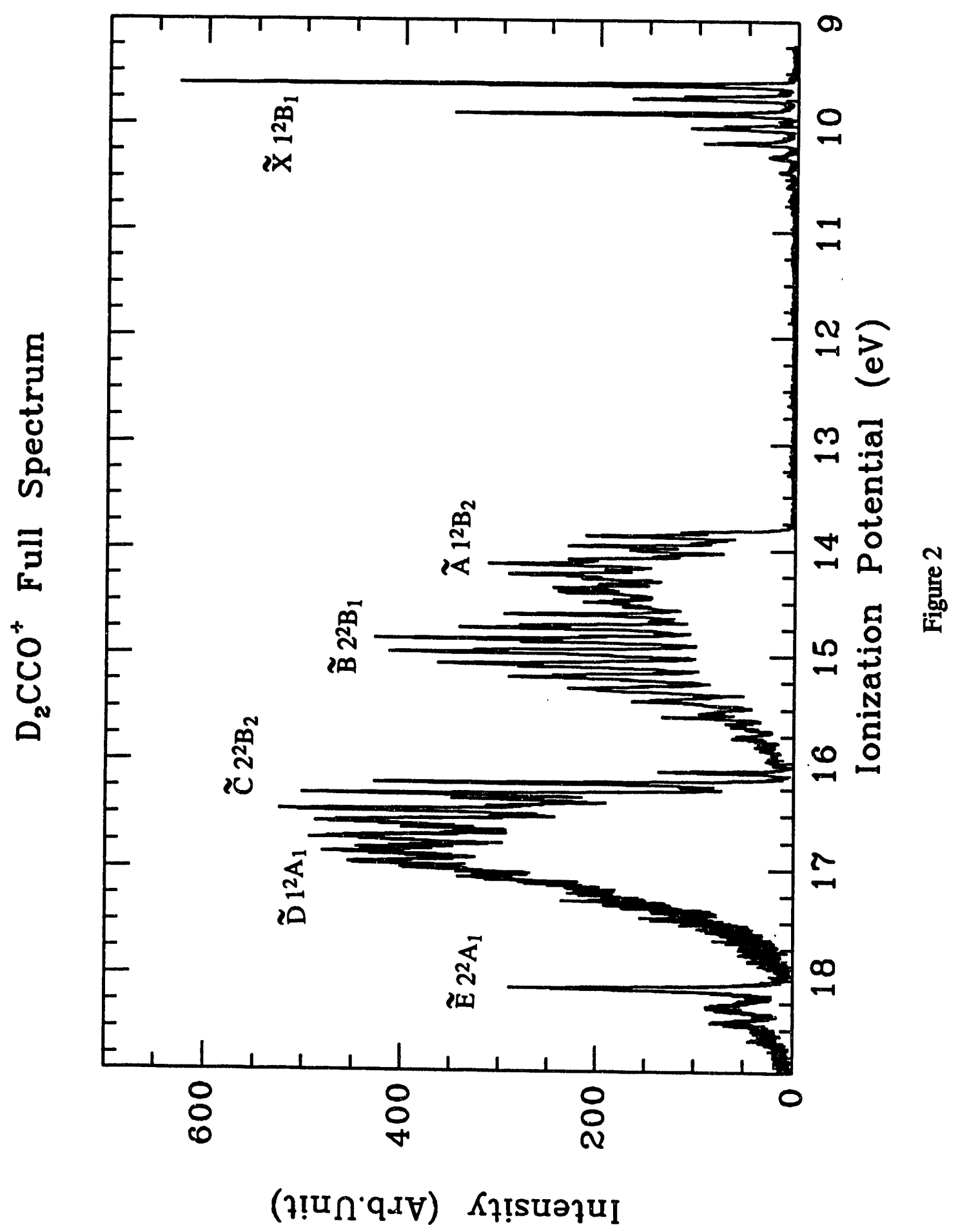




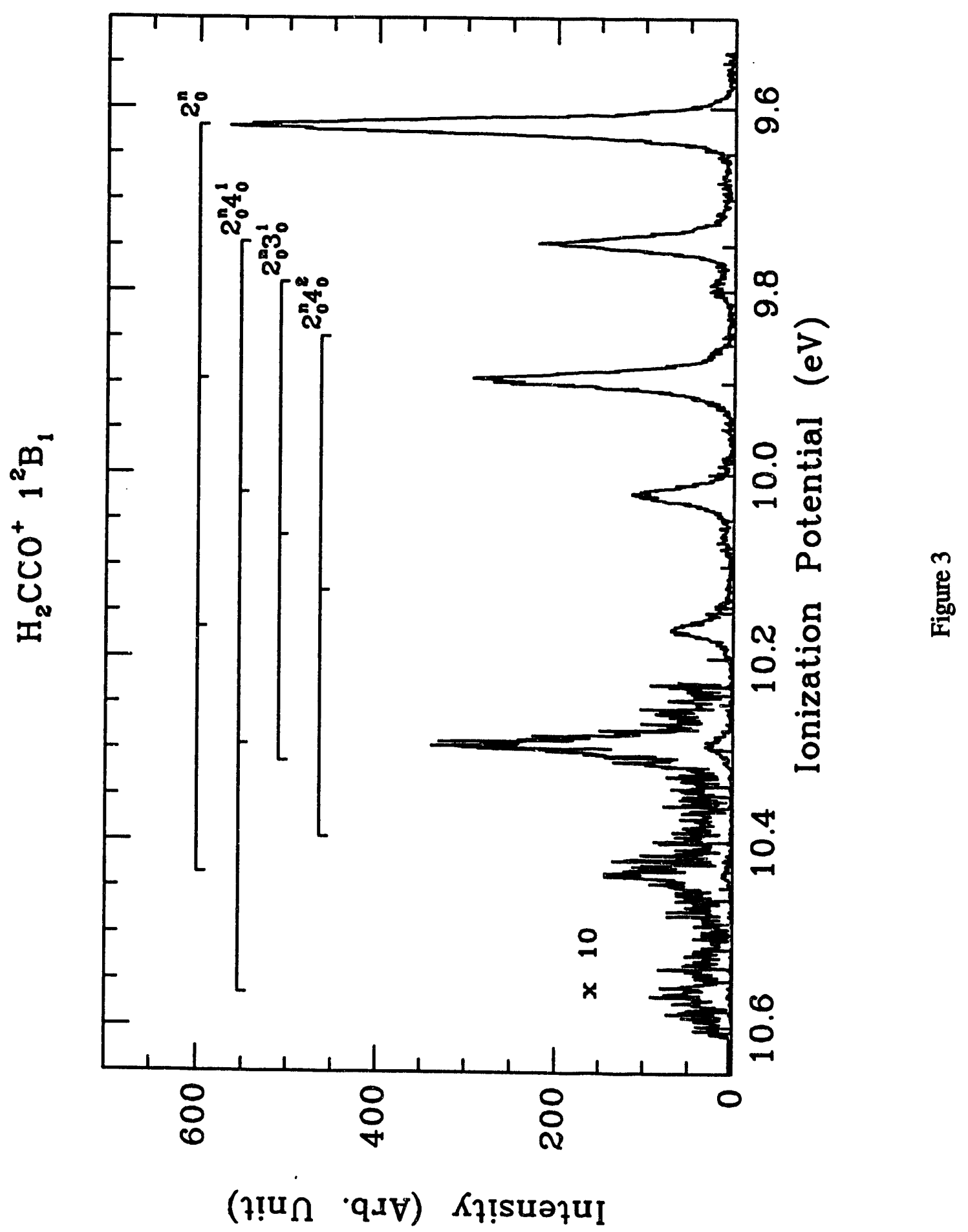




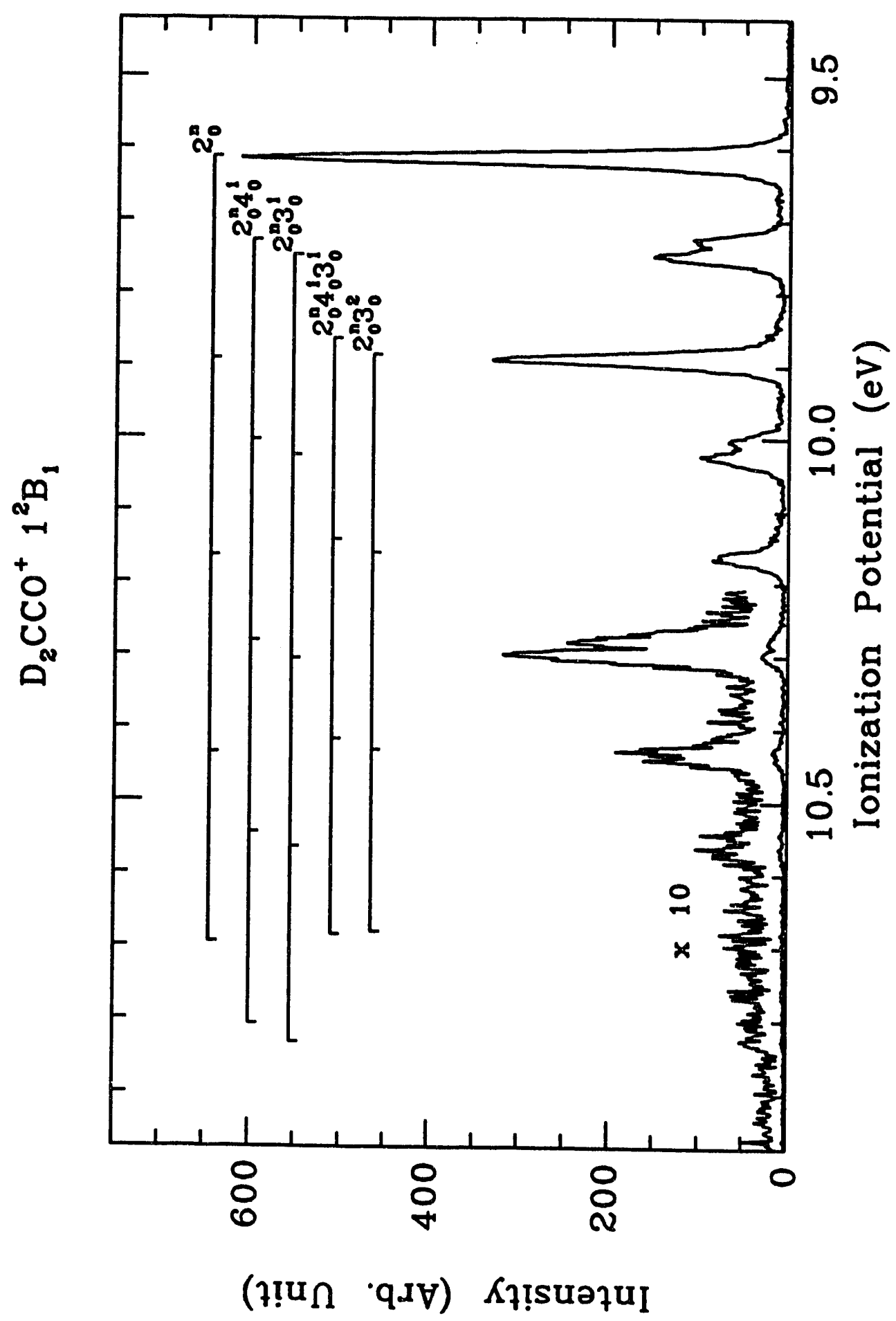

$\underset{⿱ 乛 龰}{0}$ 


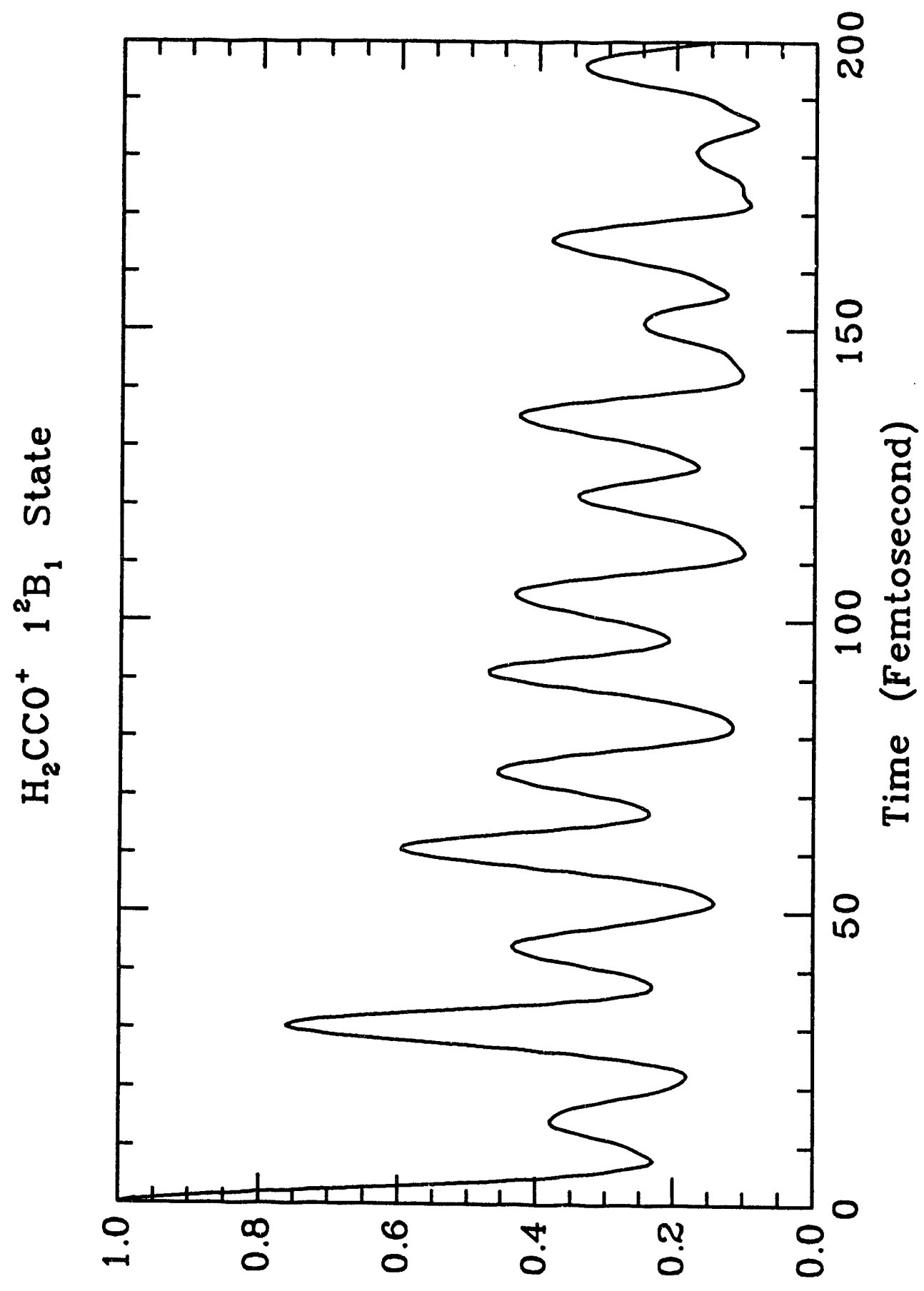

葲

(7) 0 


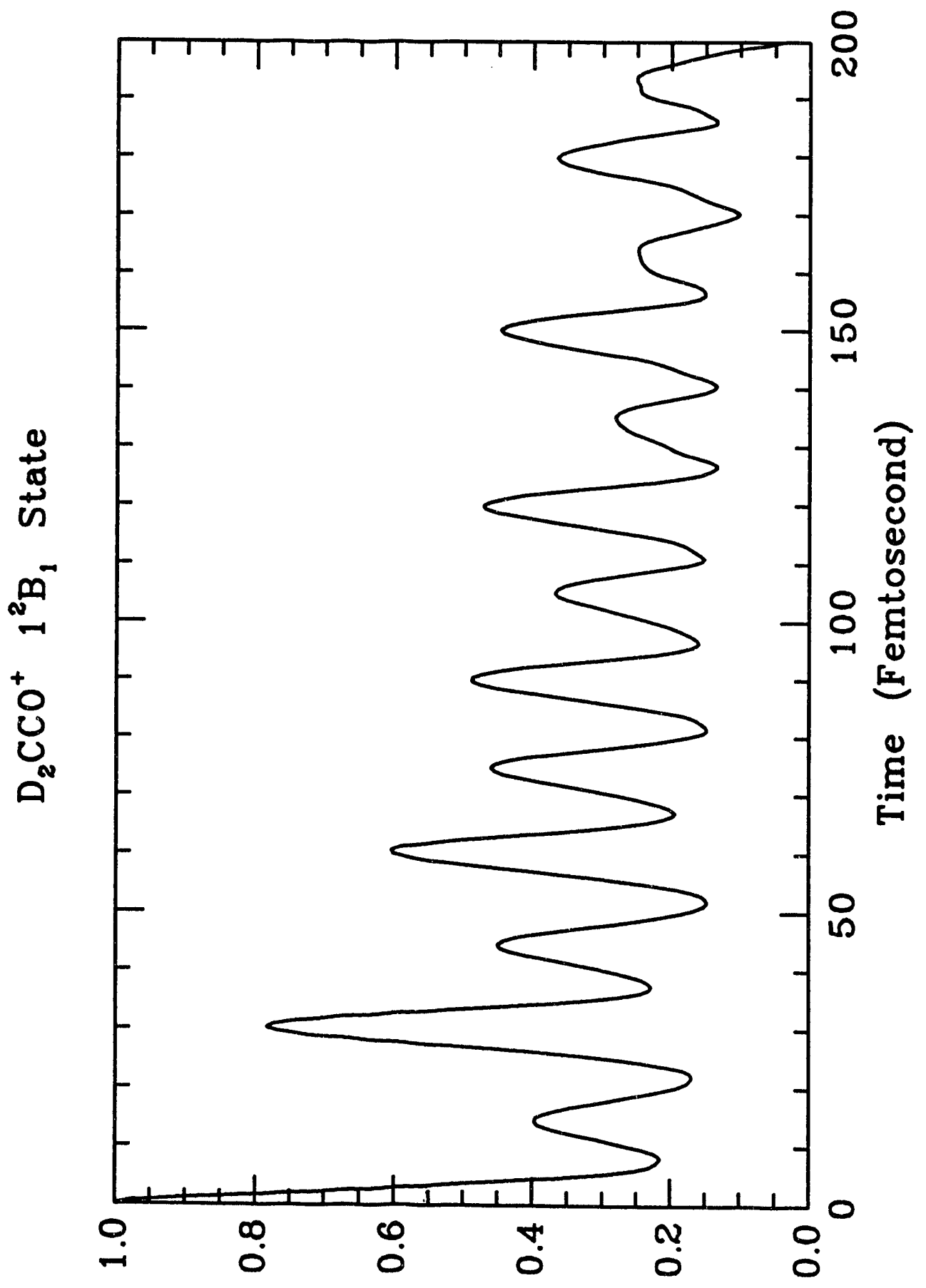

吕

(7) 


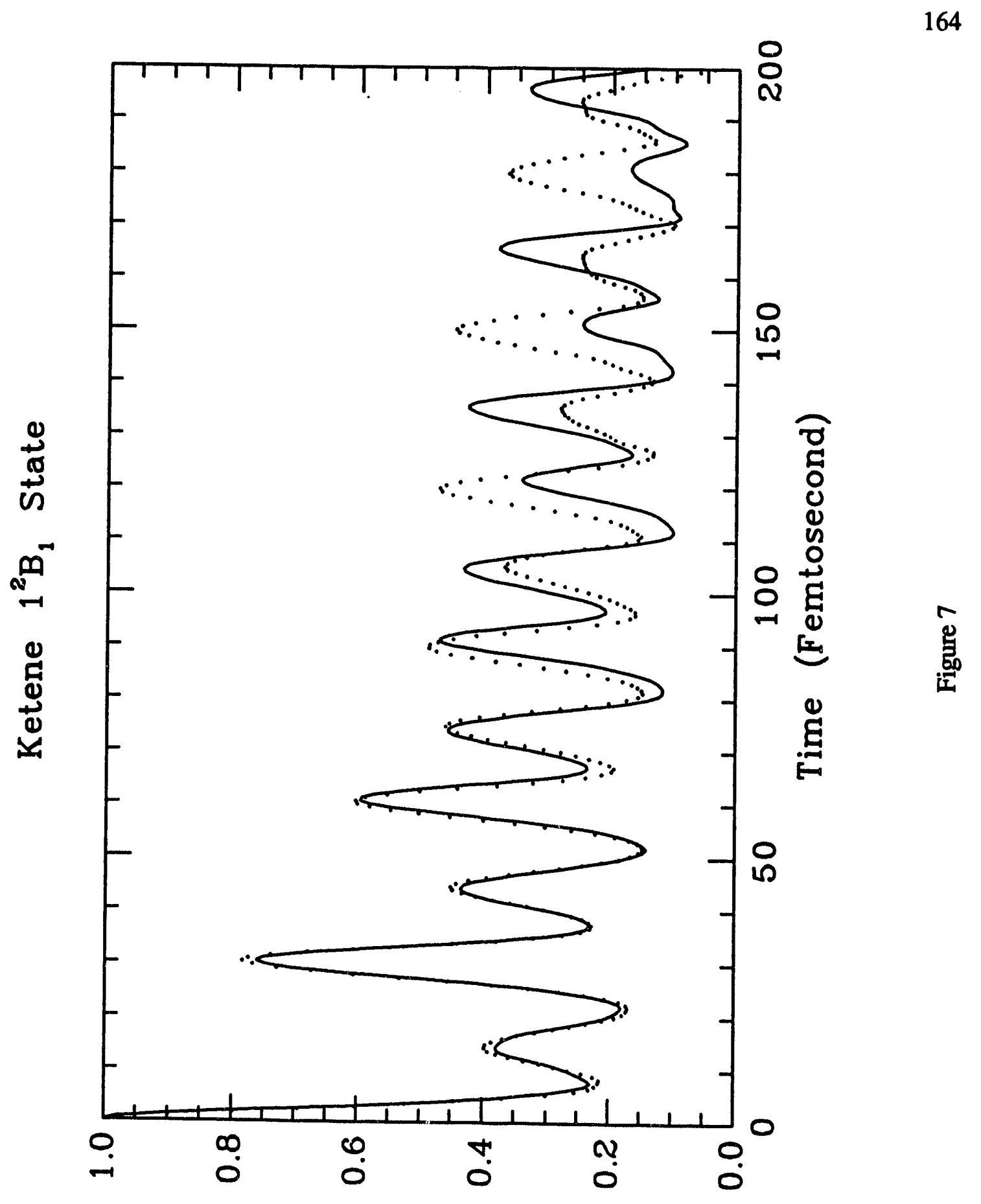

(7) 3 


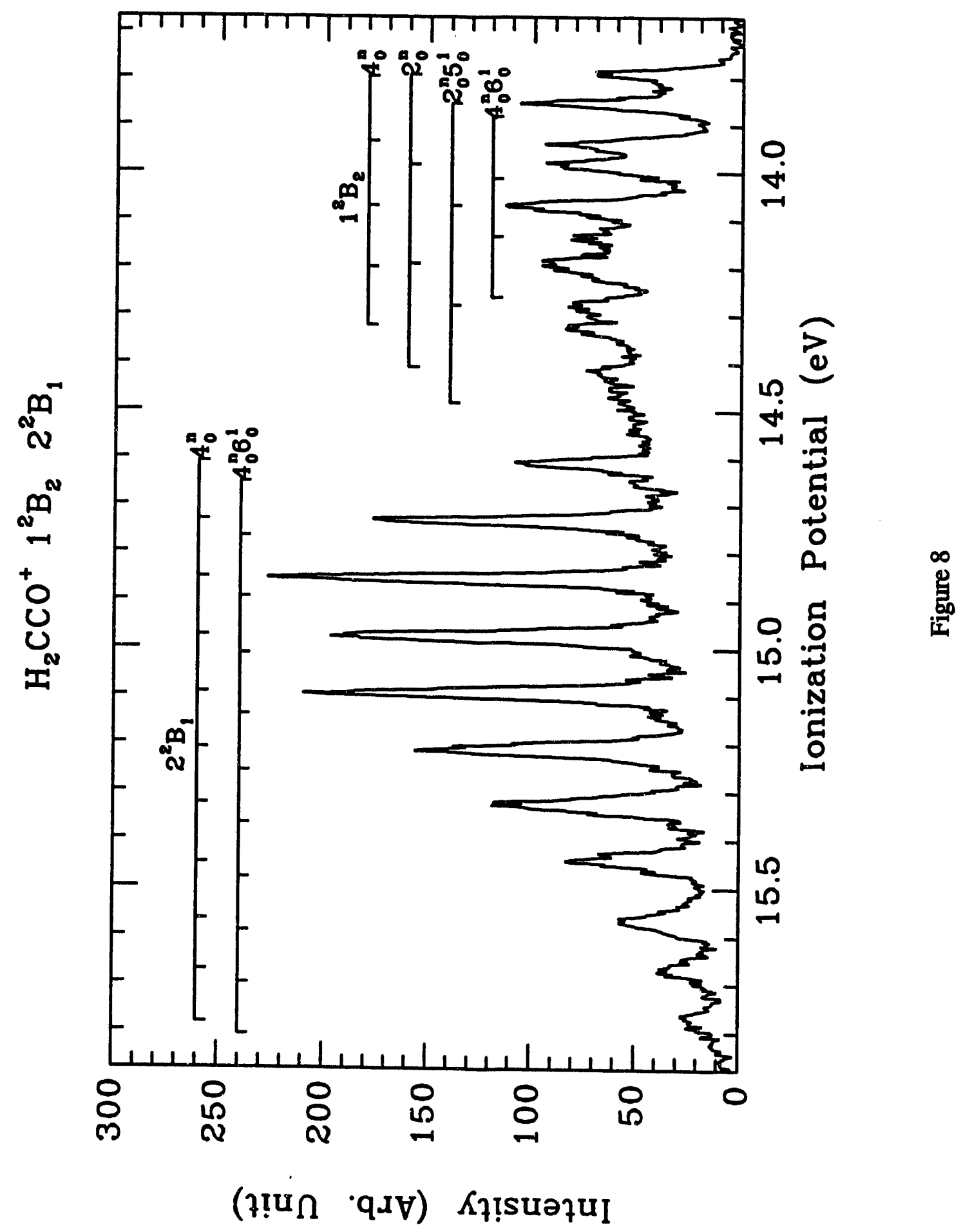




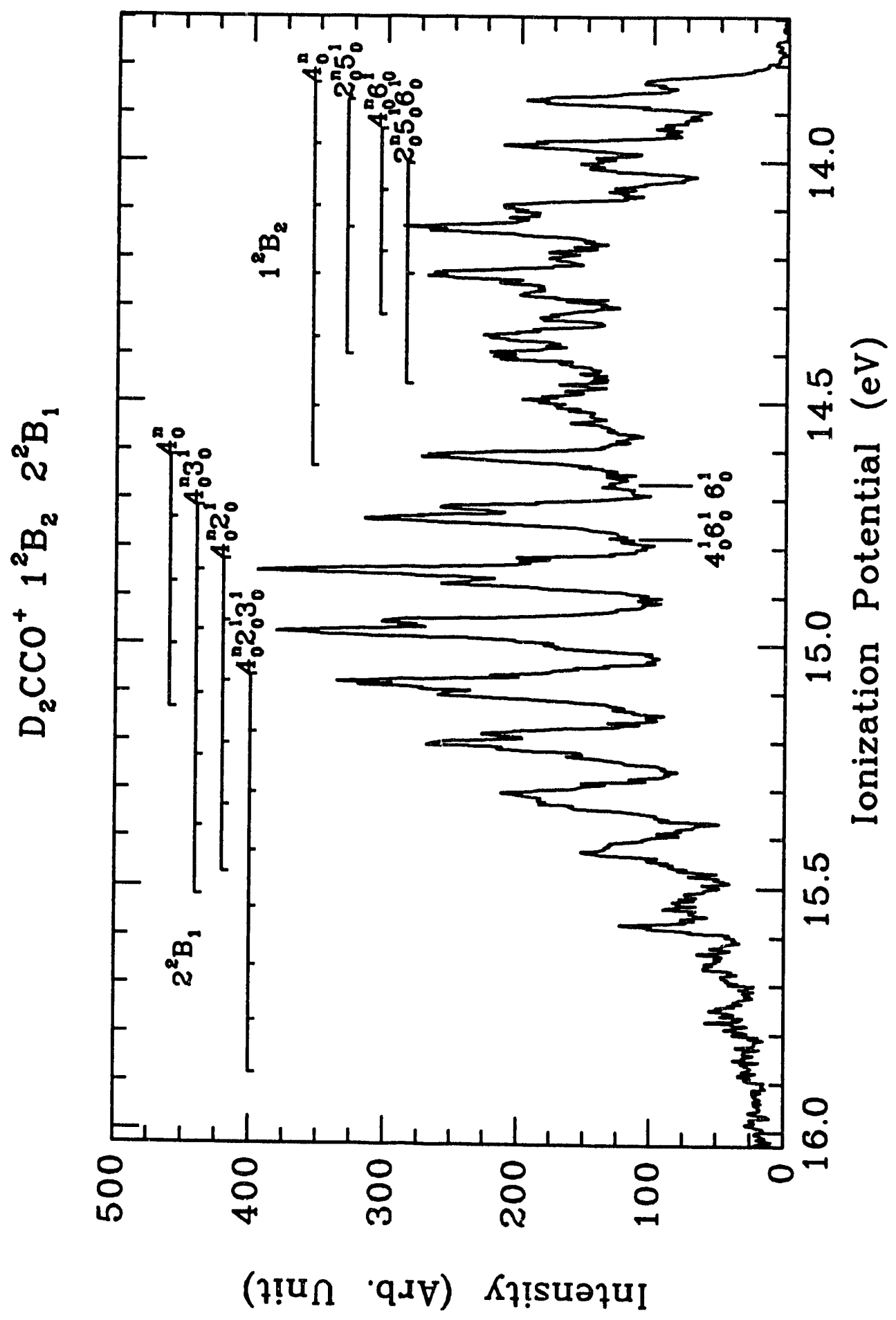

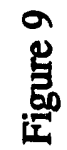




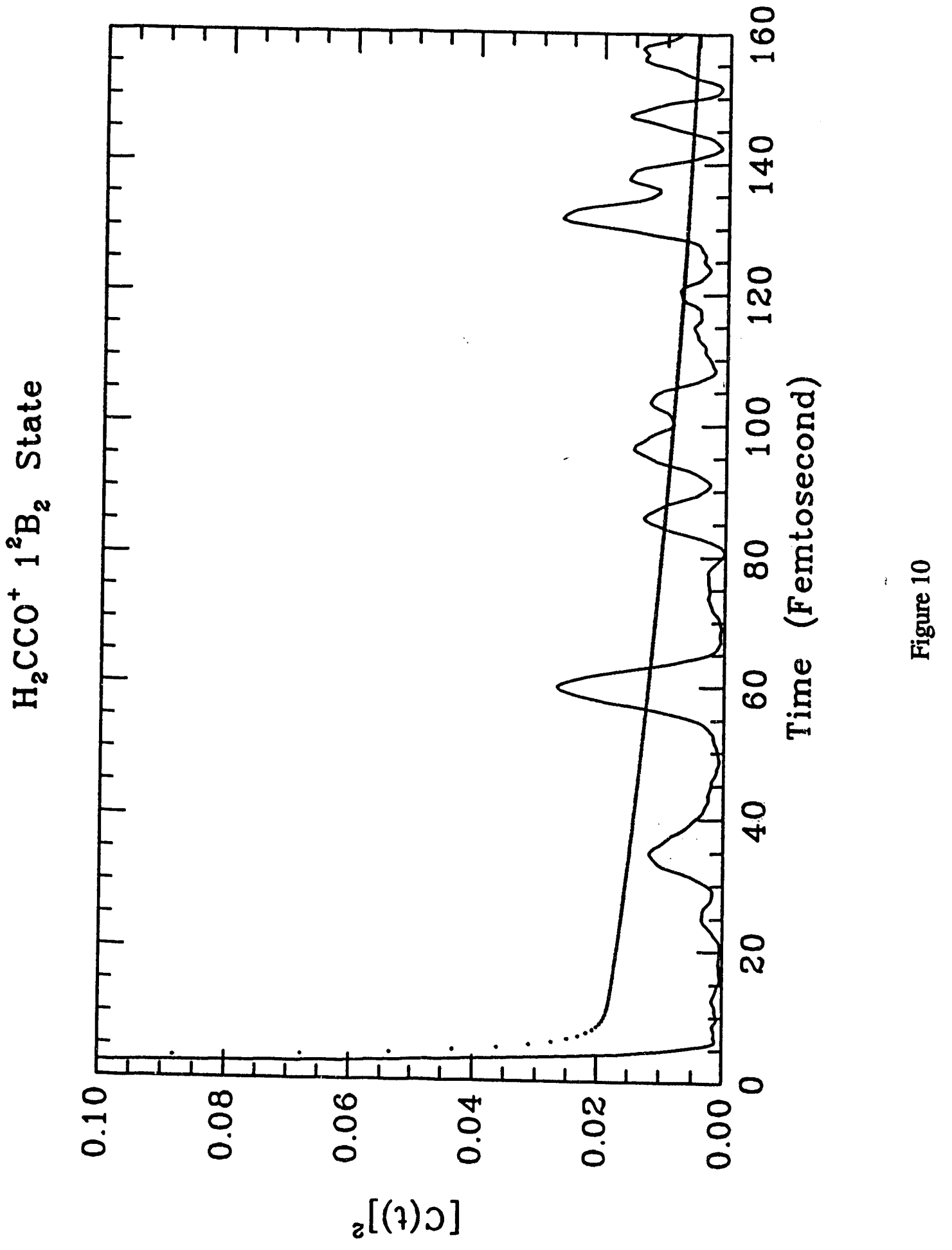




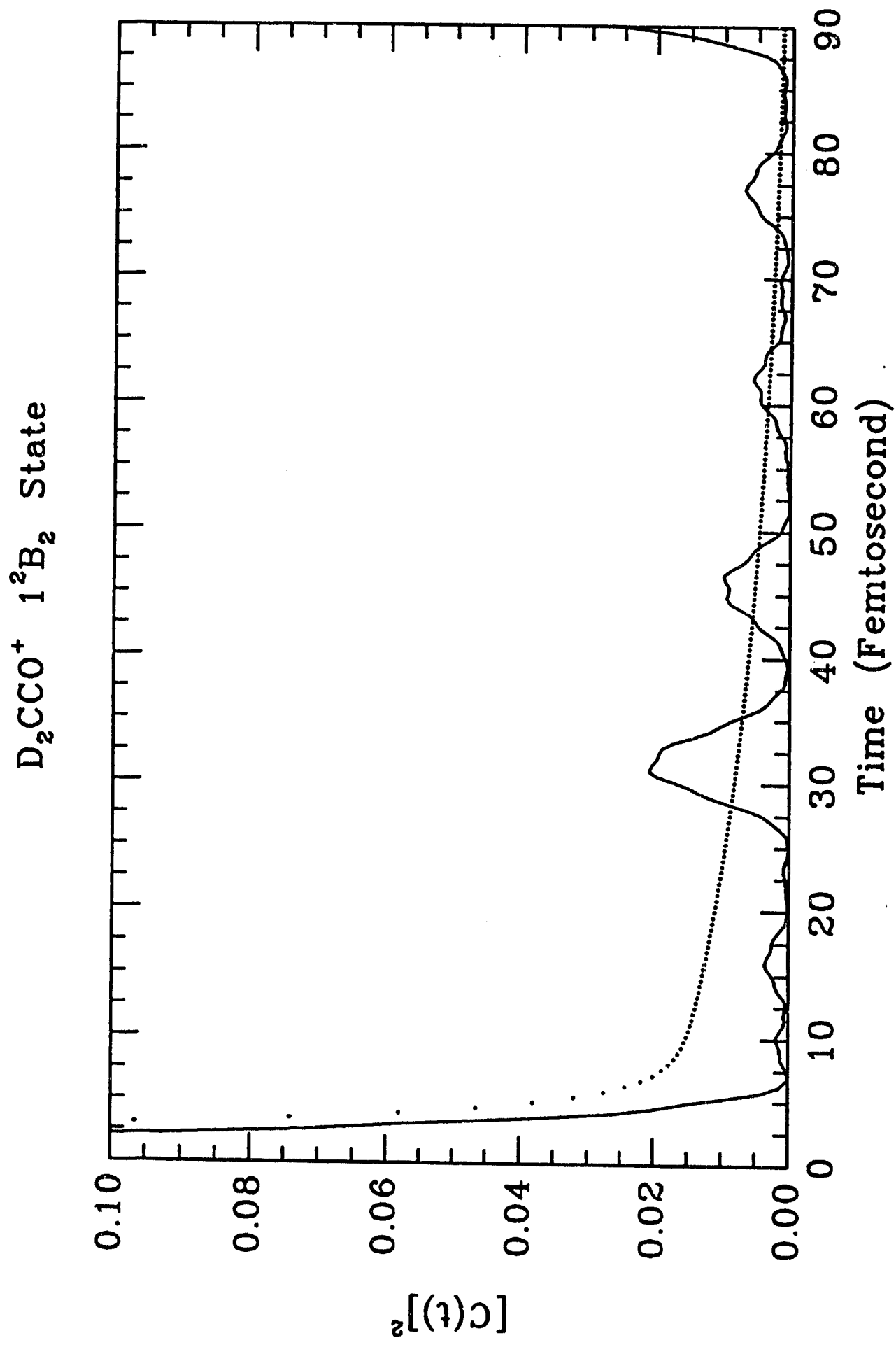

至 


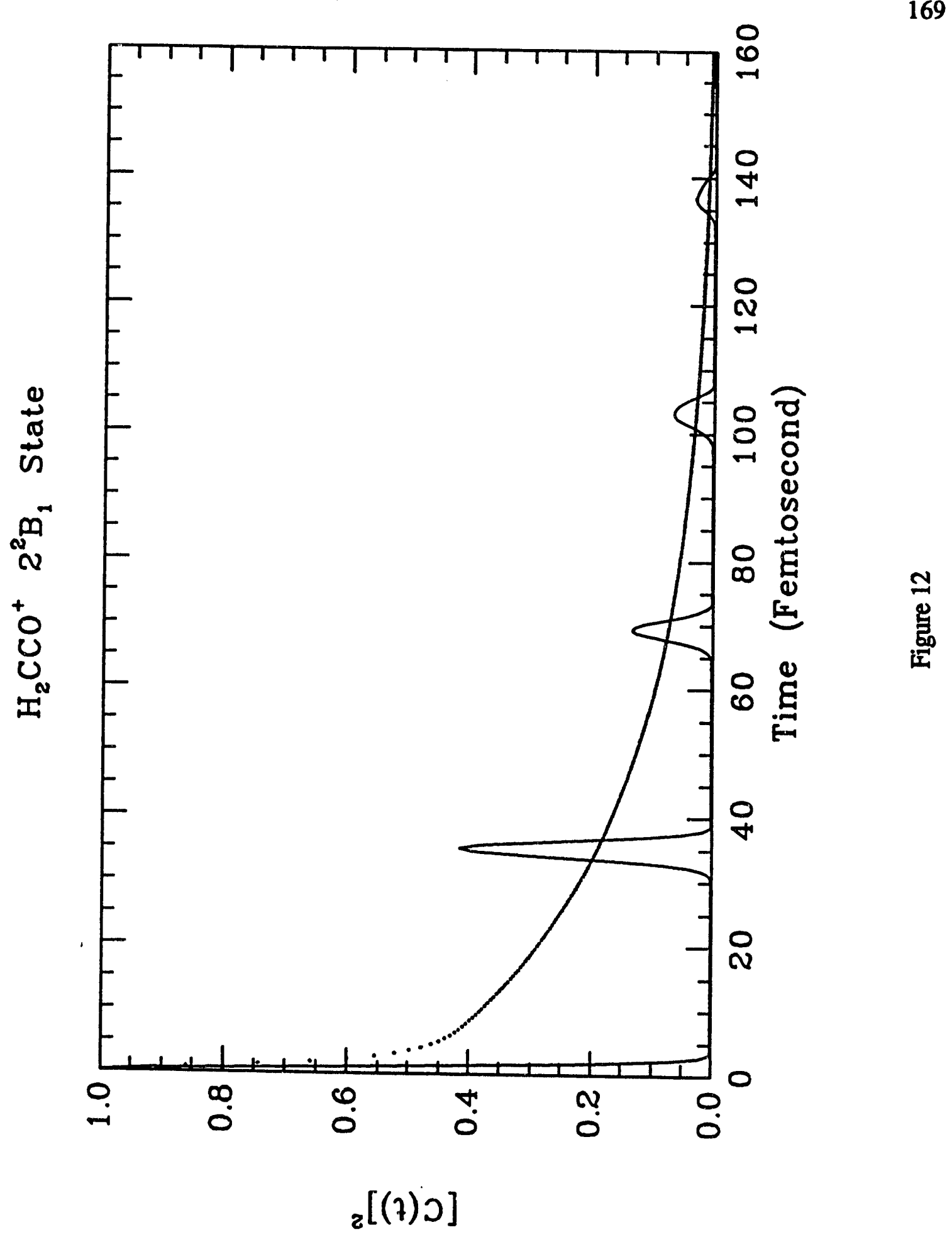




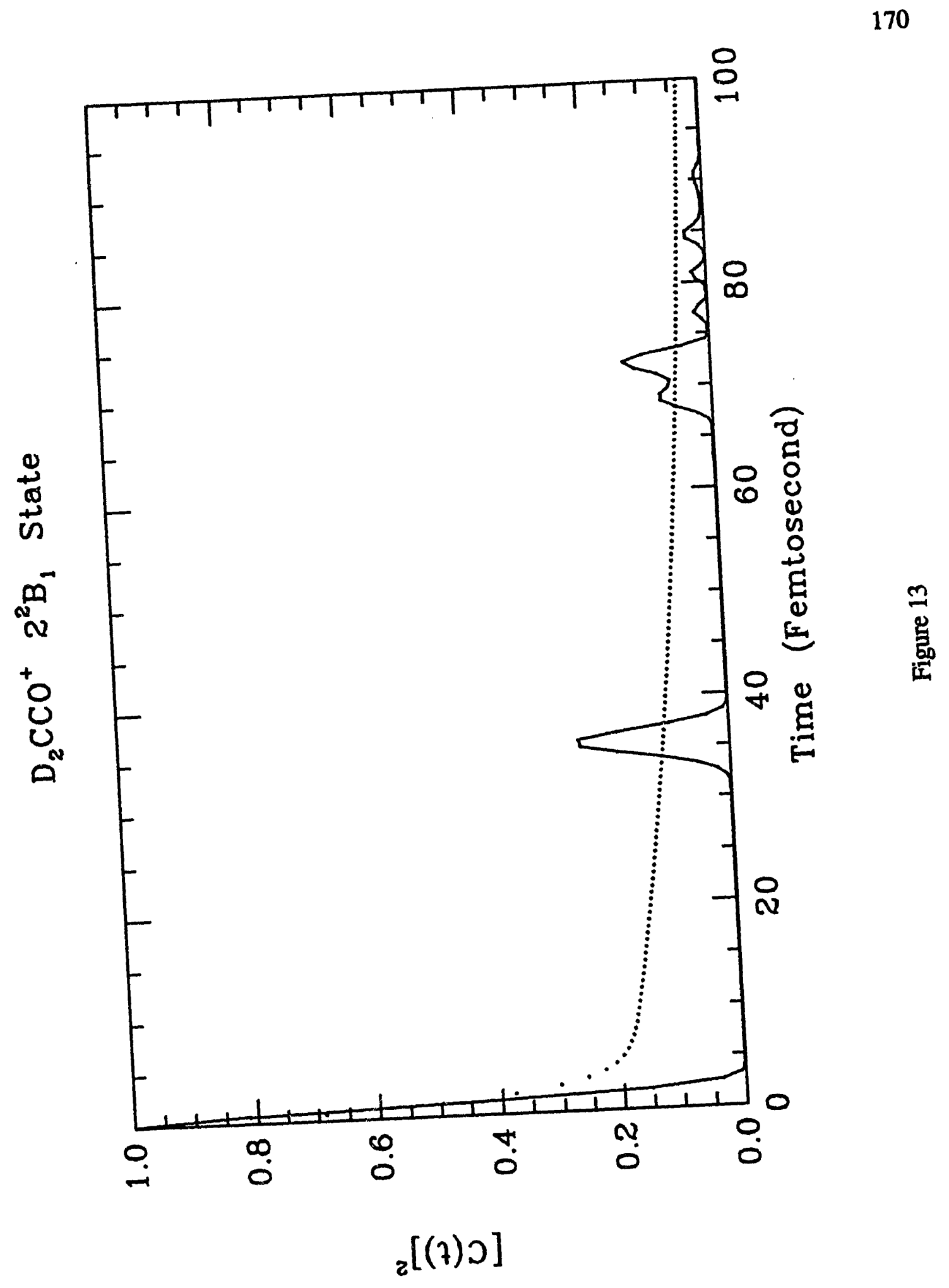




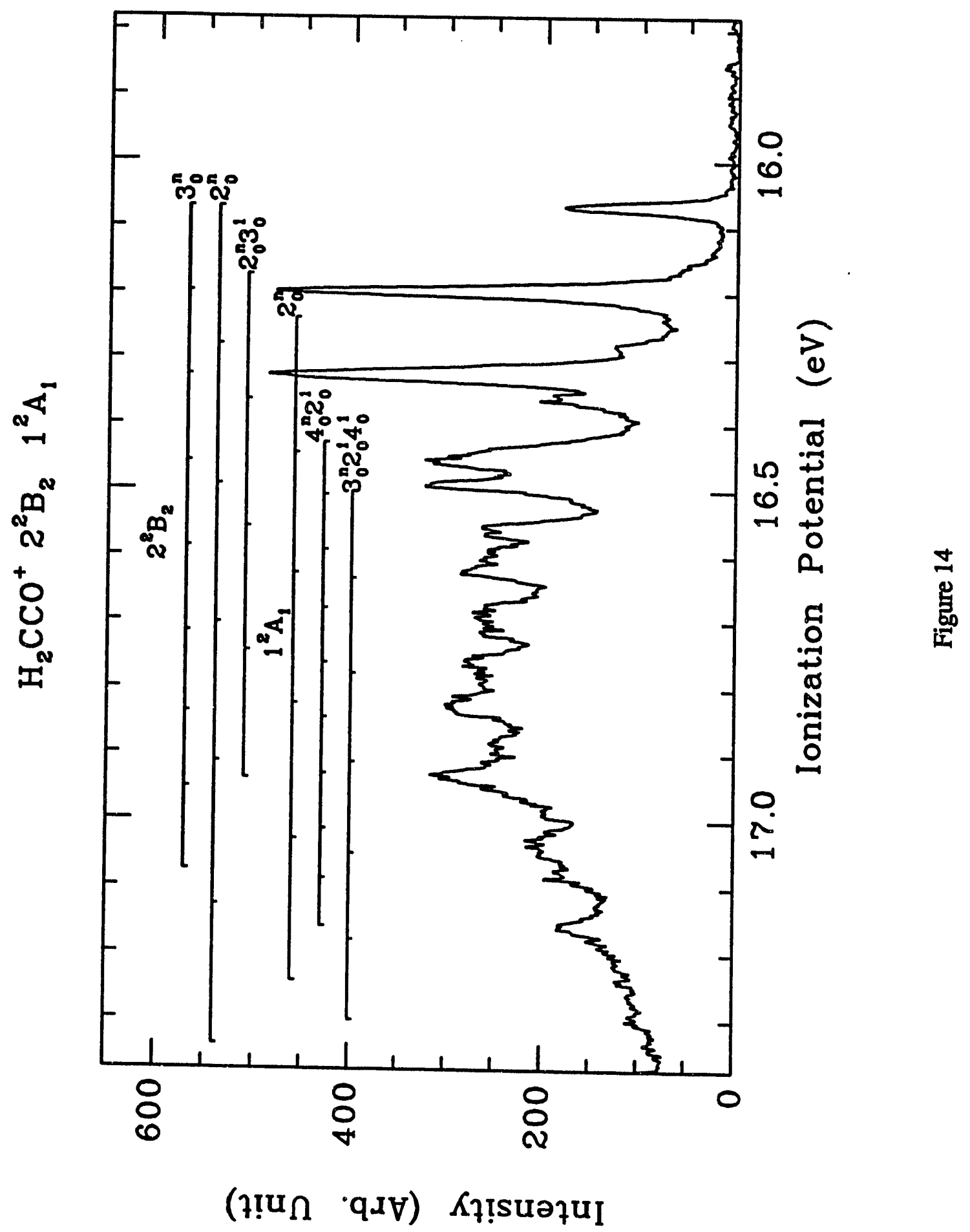




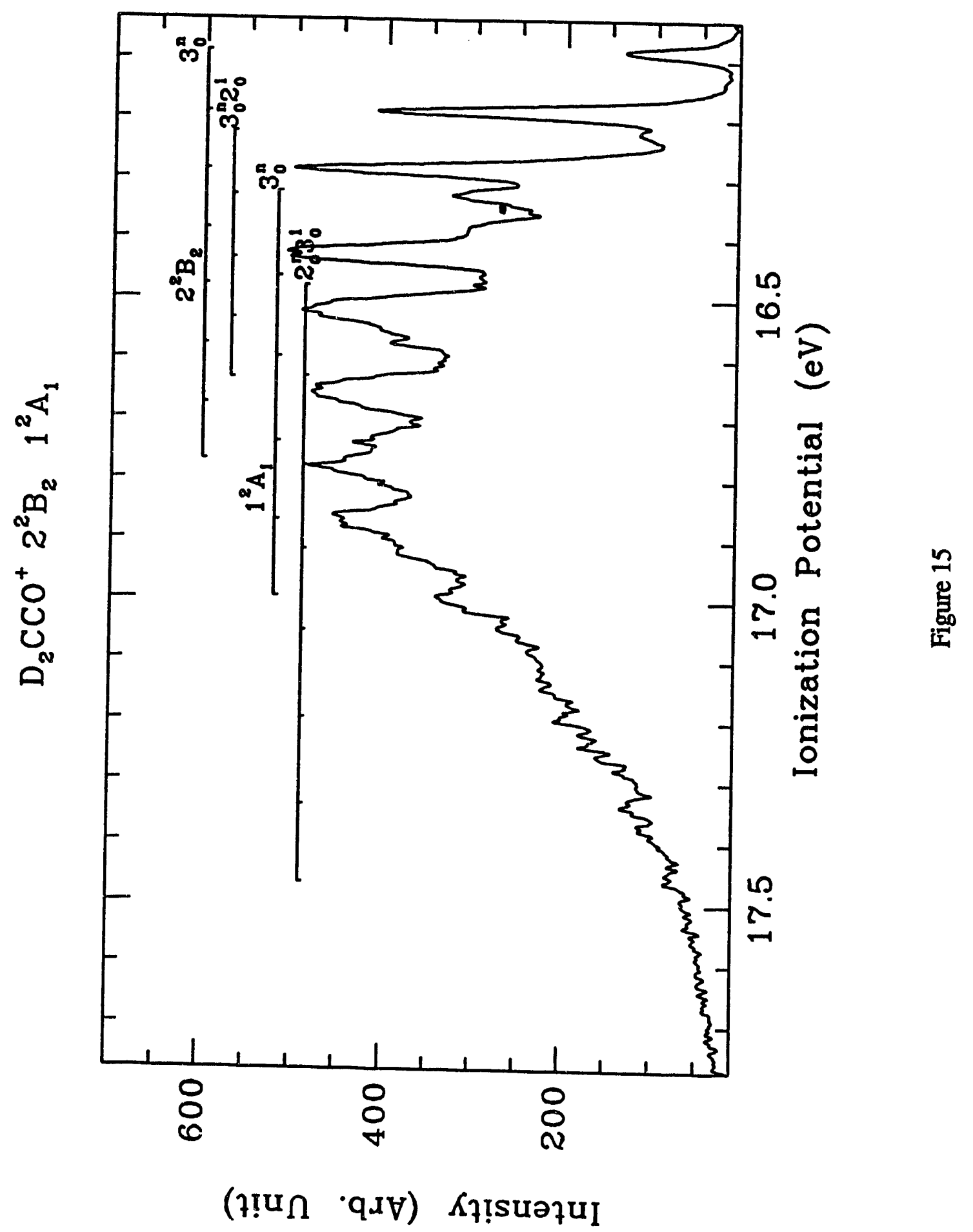




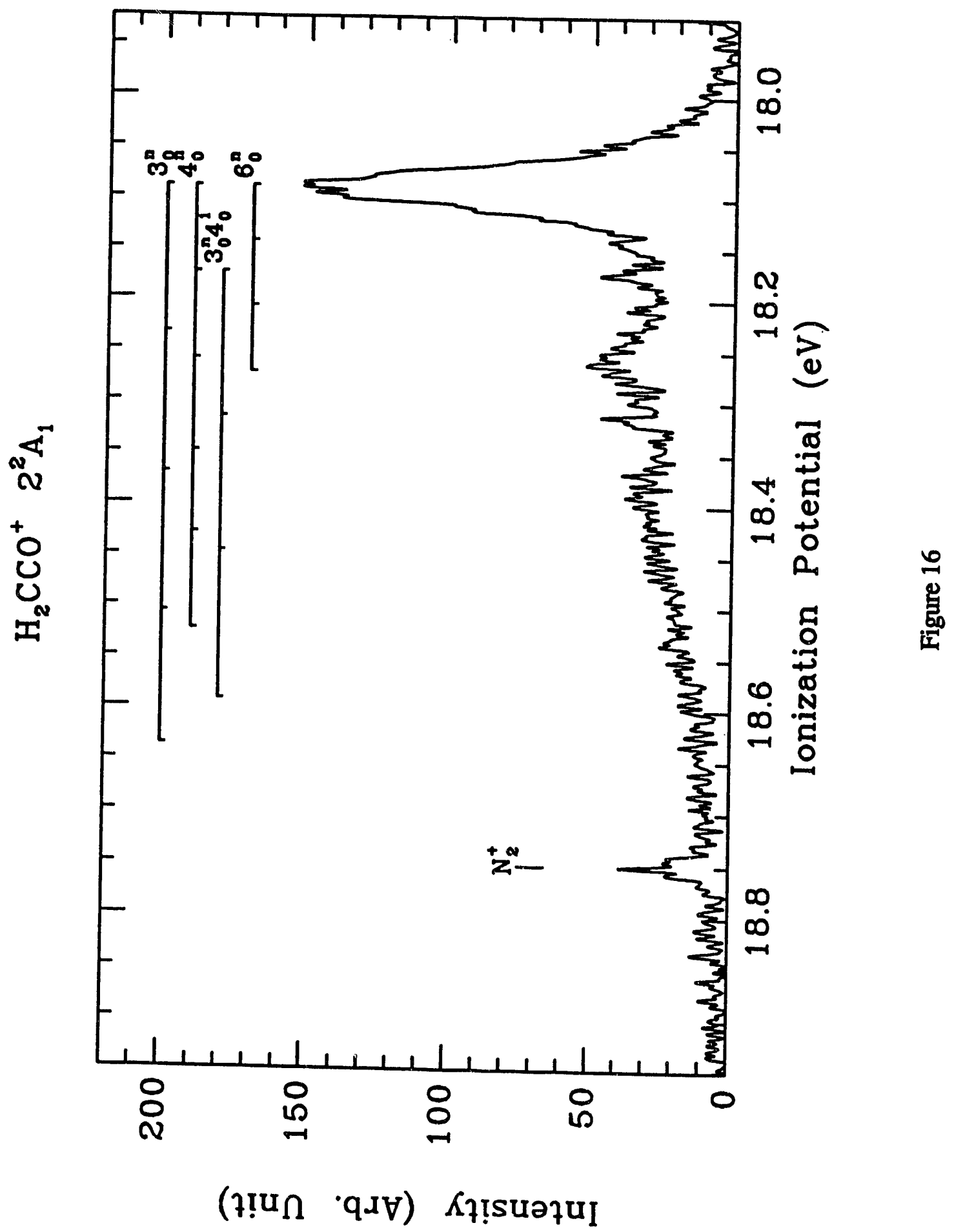




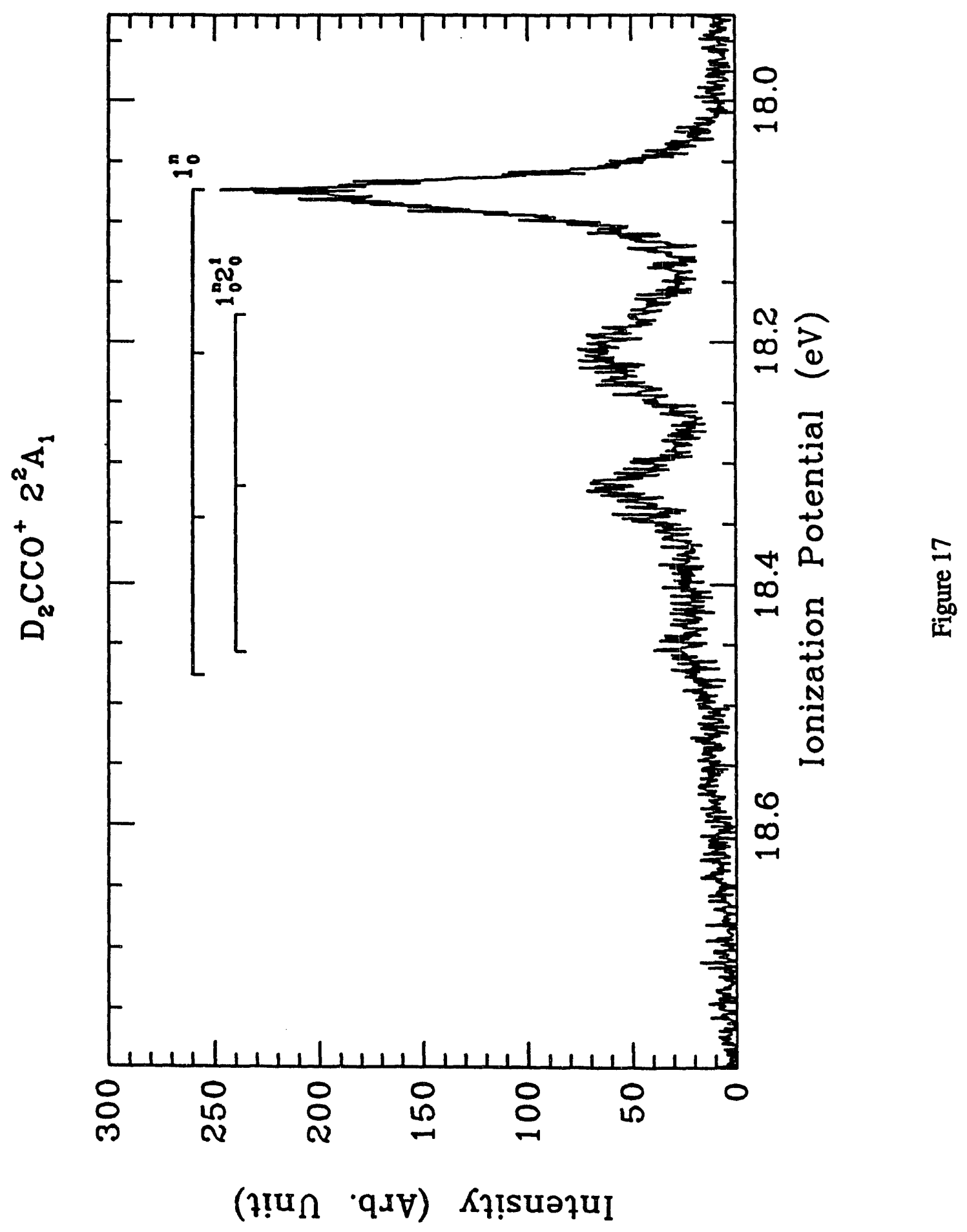




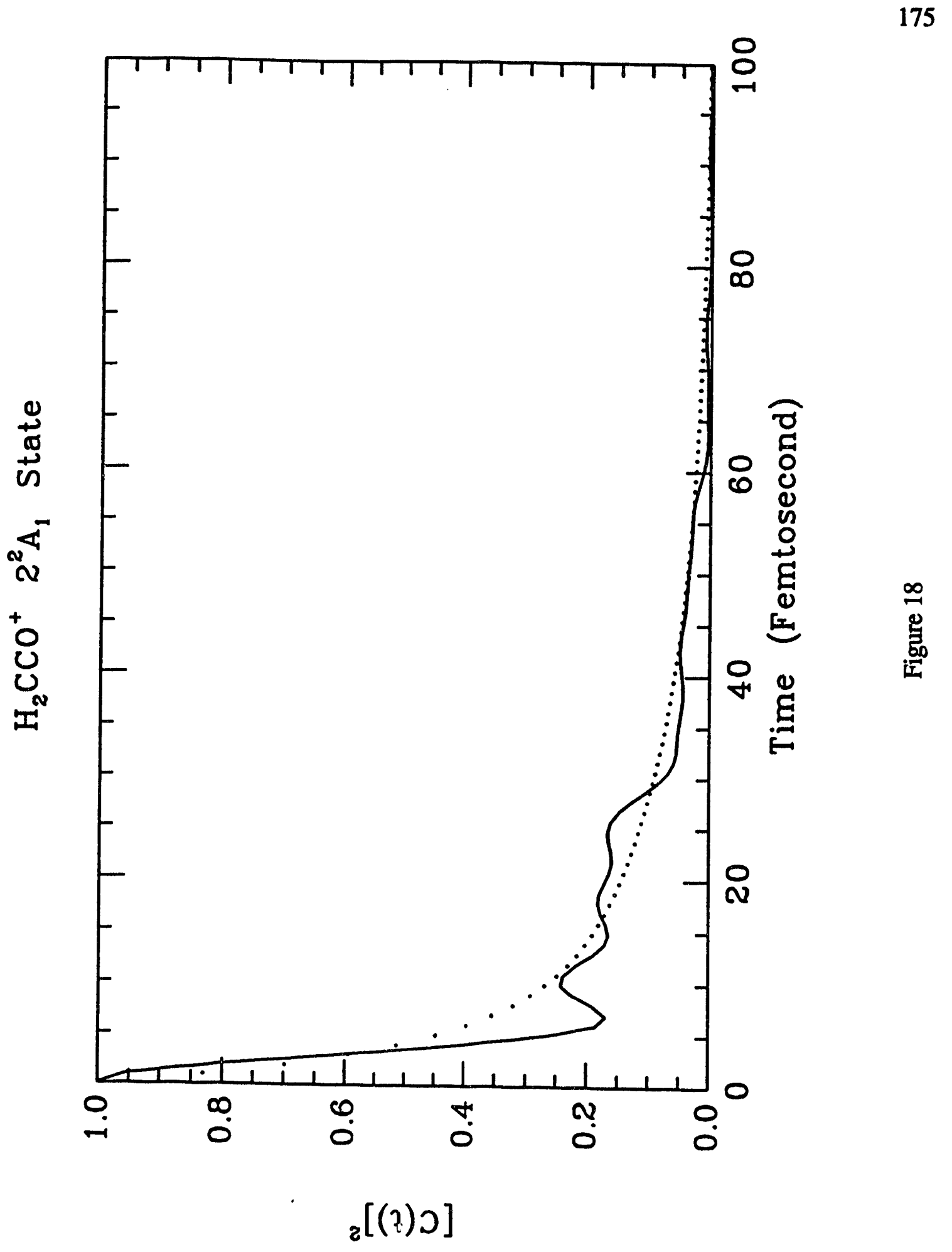




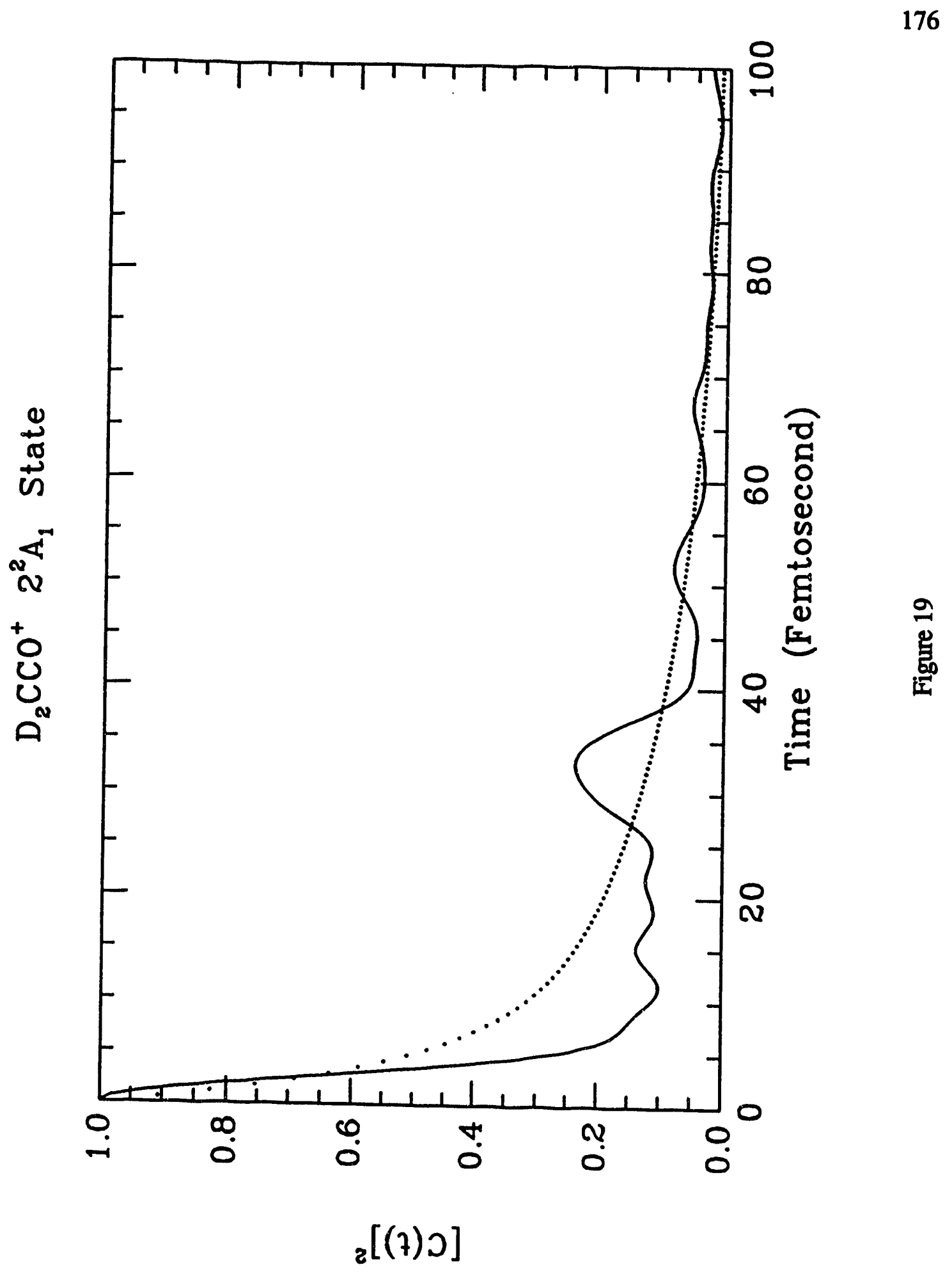




\section{Chapter Five: Conclusions, and the Future of Photoelectron}

\section{Spectroscopy}

The electronic structure, spectroscopy and the decay dynamics of polyatomic molecules have proved to be an exciting, ever-growing field where much unknown remains unchallenged even today. The photoelectron spectroscopy and intramolecular dynamics of the two systems studied in this dissertation have shown the rich information we can get from such studies.

The high-resolution spectra reported have resolved some long-standing controversies on the electronic structure of the formaldehyde and ketene cations. In the case of formaldehyde, we have shown positively with experimental evidence that formaldehyde cations in their ground and the first excited states might have non-planar equilibrium ge smetries. The fine structures on the first excited state of formaldehyde are fully resolved here for the first time. Theoretical works have long predicted that vibronic (vibrational) coupling can be very different for isotopic compounds in the same electronic state. This effect increased the difficulty substantially in the assignment of spectra even with the aid of isotope substitution. We have observed strong isotope effects on the vibronic (vibrational) coupling experimentally. In the second excited state of formaldehyde, very different vibrational progressions are observed for isotopic compounds. We hope the experimental work reported will stimulate much more work on the theoretical front to further our understanding of vibronic couplings and their effects on the spectroscopy and structures of molecules. The present study favors the many-body theoretical calculations that explain the experimentally observed spectra much better than the simple Hartree-Fock approach calculations. The vibrational autocorrelation function calculated from the photoelectron spectrum of the first excited state of formaldehyde decays rather slowly compared with the molecular vibrational motion. The decay of the first 
excited state of formaldehyde cation thus may not be as simple as we previously thought. The third excited state of formaldehyde cation, on the other hand, decays extremely fast indicating that unimolecular dissociation and other ultrafast intramolecular processes are the main decay pathways.

The photoelectron spectroscopy and intramolecular dynamic studies reported have shed considerable light on the structure and decay dynamics of ketene cations. The high resolution achieved, coupled with very effective rotational cooling by supersonic expansion revealed much more vibrational structure detail in the ground and the first excited states than previously observed. Here, again, the vibrational couplings are different in the isotopic compounds, as is apparent in the present spectra. The AIEs of the first and the fifth electronic states are determined unambigously to a much higher accuracy than previously possible. The excitation of a 'soft' mode in the first excited state of ketene cations complicated considerably the complexity of the photoelectron spectrum of this band. Resolving this 'soft' mode here for the first time gives future theoretical work much needed experimental information on this state that will elucidate some of the controversies surrounding this state. The analysis given support this state is predissociative in the Franck-Condon part of the upper potential energy surface, which is characterized by the extremely fast decay of the vibrational autocorrelation function calculated from the photoelectron spectrum. The autocorrelation functions calculated for the grounds state of ketene cations shows essentially an undisplaced wavepacket that oscillates with samll amplitude around the minimum of the upper potential energy surface, retaining high levels of correlation strength at all times. The correlation functions calculated for the fifth excited state shows ultra fast decay on the time scale of a few femtoseconds. This, together with the broad peak shapes and very short vibrational progressions observed in the photoelectron spectrum of this band, implies that this state is dissociative in the Franck- 
Condon region. The intramolecular dynamics of the ketene cations have received relatively little attention previously. The autocorrelation functions calculated can only be regarded as the very beginning of our detailed studies on the intramolecular dynamics of systems with such complexity as ketene cations.

This dissertation also aroused some interesting questions about the structure, spectroscopy and dynamics of the polyatomic molecules that need to be addressed in future theoretical and experimental works. A perspective is in order here. The high resolution achieved and the effectively rotational cooling offered by the MBPES machine are remarkable in many aspects. The coupling of a supersonic beam with the a unique double pass high resolution electron-energy analyzer is essential for this success. However, there are improvements that can be made to enhance substantially the performance of the apparatus. The first one will be the light source. The helium discharge lamp used has been optimized to produce intense, highly monochromatized light. The limit of the helium lamp was evident also. The linewidth was about $2 \mathrm{meV}$. Any efforts to improve the resolution of the analyzer must improve the performance of the light source first. In this regard, the fast advancing VUV lasers and synchrotron light source should be the primary sources for future high-resolution work. The second aspect is the electron-energy analyzer. The present analyzer succeeds fairly well around $10 \mathrm{meV}$ resolution. The state of the art in electron-energy analyzer (excluding those time-of-flight types) performance is around 1 meV resolution. Thus there is room in improving the performance of the analyzer itself. We believe this can be achieved with a larger hemispherical analyzer operating in the lowpass energy range $(2-5 \mathrm{eV})$. The magnetic shielding problem can be essentially eliminated by careful designing the $\mu$-metal magnetic shielding with as few openings as possible. The magnetic shielding of the MBPES has proved to be very effective in getting the high resolution desired. The residual magnetic field inside the analyzer chamber was measured 
to be $\sim 5 \mathrm{mGauss}$ which was largely attributed to the field penetration through openings in the $\mu$-metal, and this can be further reduced to less than $1 \mathrm{mGauss}$ without much difficulty. The distance over which electrons travel from the ionization region to the analyzer was almost $100 \mathrm{~cm}$. This was dictated by the requirements of varying the pass energy and installing the prefilter to reduce stray electrons. In practice, the analyzer was operated at fixed pass energy exclusively. Fixed pass energy requires much less sophistication in the design of the lens system, and reduces the length of the lens stacks considerably. If effective pumping of the main chamber can be achieved with better beam catching in the exhaust chamber, the stray electron problem can be minimized. This will simplify considerably the design of the lens system and the $\mu$-metal magnetic shielding. The 8:1 deceleration lens voltages have deviated substantially from the theoretical calculated values. The cause of this deviation was not clear. This affects the stability and the resolution achievable considerably. We believe that a real aperture at the virtual entrance will help stabilize the operation and improve the resolution of the apparatus with little inconvenience. In fact, the best resolution was obtained when we had a real aperture at the virtual entrance of the hemispherical analyzer. Also, it was discovered that the multichannel detector's actually usable area was only about half the active area. This is caused most probably by the stray field and improper termination of the electric field. In this regard, better field termination will increase the detection efficiency considerably. With these improvements, the new generation of MBPES should be operative with resolution in the a few meV ranges just as the present MBPES did in the $\sim 10 \mathrm{meV}$ range. The current MBPES apparatus has no trap for the diffusion pump of the beam source, and no isolation valve exists between the beam source and the main chamber. This causes contamination of the main chamber and frequent venting of the analyzer chamber. If a trap can be installed on the beam source diffusion pump, this will reduce considerably the possibilities of contaminating the main chamber. 
However, the ultimate resolution achievable in photoelectron spectroscopy depends on the light source linewidth more than the resolution of the analyzer. With the advance of threshold analyzer (time-of-flight) combined with the resolving powers offered by VUV lasers and modem synchrotron radiation source, like the advanced light source now under construction at Lawrence Berkeley Laboratory, photoelectron spectroscopy will eventually have the resolution now enjoyed by optical spectroscopy. This will enable us to study the details of electronic structures, spectroscopy and dynamics of molecular cations in the near future. Rotationall $y$ resolved photoelectron spectroscopy is the ultimate goal in this regard, and it is not far from realization.

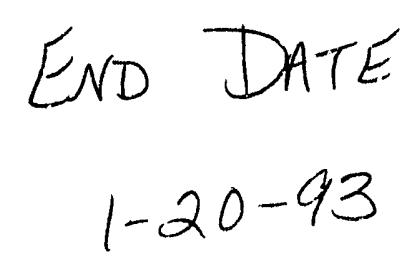

